(C) 2011

Daryl Curtis Hare

All Rights Reserved 


\title{
MEASUREMENTS OF THE TOP ANTI-TOP PRODUCTION CROSS SECTION AND TOP QUARK MASS IN THE HADRONICALLY DECAYING TAU + JETS DECAY CHANNEL AT CDF
}

BY DARYL CURTIS HARE

\author{
A dissertation submitted to the \\ Graduate School-New Brunswick \\ Rutgers, The State University of New Jersey \\ in partial fulfillment of the requirements \\ for the degree of \\ Doctor of Philosophy \\ Graduate Program in Physics and Astronomy \\ Written under the direction of \\ Prof. Eva Halkiadakis \\ and approved by
}

New Brunswick, New Jersey

October, 2011 


\author{
Abstract of the Dissertation
}

\title{
Measurements of the Top Anti-Top Production Cross Section and Top Quark Mass in the Hadronically Decaying Tau + Jets Decay Channel at CDF
}

\author{
By Daryl Curtis Hare \\ Dissertation Director: Prof. Eva Halkiadakis
}

In this thesis, we present the first exclusive observation of the $t \bar{t} \rightarrow$ hadronic $\tau+$ jets decay channel. Using these events, we measure the $t \bar{t}$ pair production cross section and the top quark mass in $2.2 \mathrm{fb}^{-1}$ of data collected with the Collider Detector at Fermilab (CDF). The Tevatron accelerator at Fermilab provides collisions of protons and anti-protons at a center-of-mass energy of $\sqrt{s}=1.96 \mathrm{TeV}$ and is one of only two accelerators in the world with enough energy to produce top quarks. With a branching fraction of nearly $10 \%$, the hadronic $\tau+$ jets decay channel is the third largest $t \bar{t}$ decay mode, and it has only been minimally explored. This the first measurement of the $t \bar{t}$ pair production cross section in this decay channel at CDF and the first measurement of the top quark mass in this decay channel in the world. The analysis introduces a new method to recover the total momentum of the $\nu$ produced in the $\tau$ decay and an artificial neural network to reduce the contribution from the largest background source, QCD multijet background. The $t \bar{t}$ pair production cross section is extracted by minimizing a negative log likelihood function which compares the number of observed events to the number of expected events for a given $t \bar{t}$ cross section. The top quark mass is extracted by minimizing a negative log likelihood function built from signal and 
background probabilities which are based on the matrix elements for $t \bar{t}$ production and decay and $W+4$ parton production, respectively. Using events selected with exactly 1 hadronically decaying $\tau$, exactly 4 jets with at least 1 identified as having originated from a $b$ quark, and large missing transverse energy, we measure the $t \bar{t}$ pair production cross section to be $8.8 \pm 3.3$ (stat.) \pm 2.2 (syst.) pb and the top quark mass to be $172.7 \pm 9.3$ (stat.) \pm 3.7 (syst.) GeV. We find both values to be in good agreement with previous measurements in other $t \bar{t}$ decay channels, and the cross section to be consistent with next-to-leading order theoretical predictions. 


\section{Acknowledgements}

First I'd like to thank my advisor Eva Halkiadakis for her guidance and expertise. I also owe thanks to several members of the Rutgers University high energy physics group: Amit Lath and Anton Anastassov, for their $\tau$ expertise; Scott Thomas, for always being willing to discuss ideas for improving an analysis; Sourabh Dube and Jared Yamaoka, for their help as I first started working at CDF; and Ian Laflotte and Julia Gonski for the many hours of help they have given to this analysis.

I also owe thanks those who were involved in the matrix element analysis: Brian Mohr, who introduced the MEAT analysis to me and found time while preparing for his Ph.D defense to teach me about the method; Florencia Canelli, who offered advice and guidance; and Jacob Linacre and Sasha Golossanov, who put in the hours next to me on the lepton + jets matrix element analysis. Of course, that never would have been possible without the donation of computing farm time from Ricardo Eusebi, Doug Glenzinski, Andy Hocker, Mousumi Datta, and any others I apologize for forgetting to mention.

I thank all the top group conveners and mass group subconveners who helped review and guide the various stages of the analysis. Particularly though, I owe thanks to Fabrizio Margaroli, Tom Schwarz, George Velev, and YenChu Chen for overseeing the $\tau$ analysis. Additional thanks go out to our internal reviewers Pasha Murat and HyunSu Lee. I also have to acknowledge Tom Schwarz again for his help with the background estimation and cross section measurement.

I'd be remiss without acknowledging all the people who work hard everyday to keep

the Tevatron accelerator and CDF detector functioning. The number is simply too great to list.

Last but far from least, I want to thank my family for all their support: my parents 
Connie and Curt, for supporting my goals from an early age; my wife Kate, for standing by me during the best and worst times; my father-in-law and mother-in-law Paul and Karen, for all the help they have given Kate and I to make this possible; my beautiful daughter Ivy, who makes all the work worthwhile; and my dog Beauty Quark, for balancing me out after spending all day thinking about top quarks. 


\title{
Dedication
}

\author{
To my wife Kate,
}

who has been by my side for this journey since the first year of graduate school. 


\section{Table of Contents}

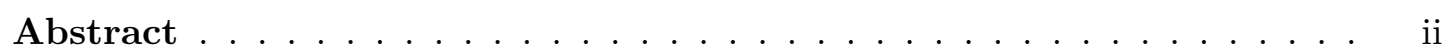

Acknowledgements .................... iv

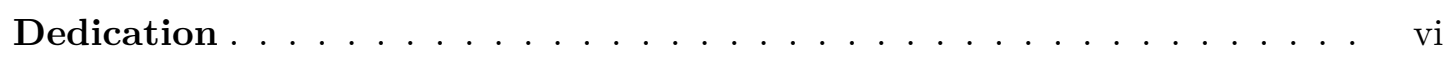

List of Tables . . . . . . . . . . . . . . . . . . . xi

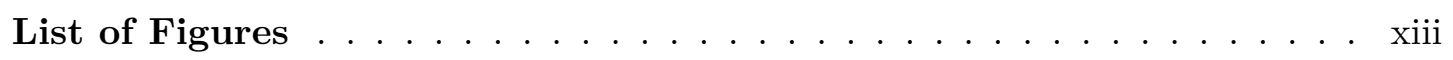

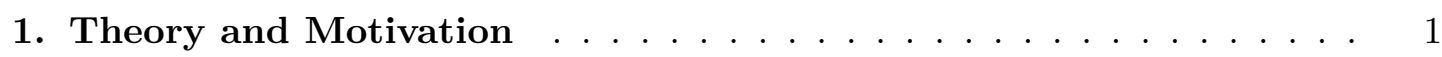

1.1. The Standard Model . . . . . . . . . . . . . . . . . . . . 2

1.2. Matrix Elements . . . . . . . . . . . . . . . . 9

1.3. Top Quark . . . . . . . . . . . . . . . . . . 9

1.4. $\tau$ Leptons . . . . . . . . . . . . . . . . . . . . 15

1.5. Overview of Chapters . . . . . . . . . . . . . 17

2. The Tevatron and the CDF Detector . . . . . . . . . . . . . . . . . 18

2.1. The Tevatron Accelerator . . . . . . . . . . . . . . . . 19

2.2. The CDF Detector at Fermilab . . . . . . . . . . . . . . . 22

2.2.1. Coordinate System . . . . . . . . . . . . . . . 25

$2.2 .2 . \quad$ Silicon Tracking . . . . . . . . . . . . . . . 26

2.2.3. Central Outer Tracker . . . . . . . . . . . . . . . . 27

2.2.4. Calorimeters . . . . . . . . . . . . . . . . . . . . 28

2.2.5. Muon Detection Systems . . . . . . . . . . . . . . 32

2.2.6. Luminosity Counters . . . . . . . . . . . . . . . 34

2.2.7. Trigger System . . . . . . . . . . . . . . . . 35 


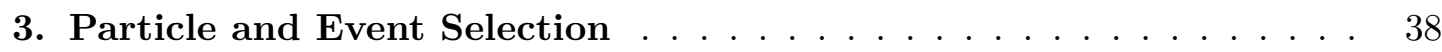

3.1. Event Trigger . . . . . . . . . . . . . . . . . . . . . 38

3.2. Particle Detection and Reconstruction . . . . . . . . . . . . . 41

3.2.1. Taus . . . . . . . . . . . . . . . . 41

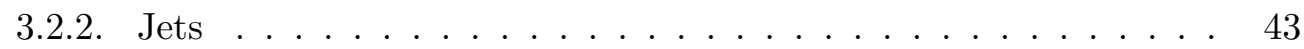

Jet Energy Corrections . . . . . . . . . . . . . . . . . . 45

Heavy Flavor Jets and b-tagging . . . . . . . . . . . . . . . 48

3.2.3. Neutrinos . . . . . . . . . . . . . . . . . . . . . 49

3.2.4. Lepton Veto . . . . . . . . . . . . . . . . . . . . . . 51

3.3. Event Selection Requirements . . . . . . . . . . . . . . . . . . 53

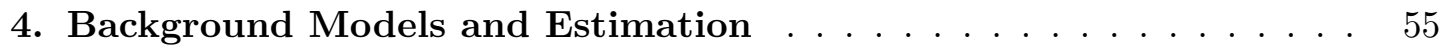

4.1. Monte Carlo Samples . . . . . . . . . . . . . . . . . . . . . . . . 56

4.2. Data Driven QCD Multijet Estimate . . . . . . . . . . . . . 56

4.2.1. Neural Network for Reduction of QCD Multijet Background. . . 59

4.2.2. Neural Network Optimization . . . . . . . . . . . . . . . 67

4.3. Background Estimate $\ldots \ldots \ldots \ldots \ldots \ldots \ldots$

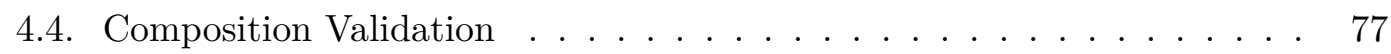

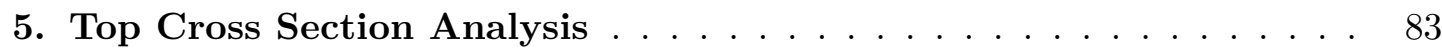

5.1. Cross Section Measurement Method . . . . . . . . . . . . . . . . 83

5.2. Acceptance and Efficiency for $t \bar{t} \rightarrow \tau+$ jets $\ldots \ldots \ldots \ldots \ldots$

5.3. Fitting for $\sigma_{t \vec{t}} \ldots \ldots \ldots \ldots \ldots \ldots \ldots \ldots \ldots \ldots \ldots$

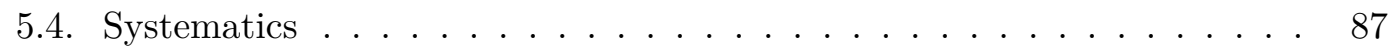

5.4.1. Acceptance Uncertainties . . . . . . . . . . . . . . . 88

Jet Energy Scale . . . . . . . . . . . . . . . . . . . . . . . 89

Initial and Final State Radiation . . . . . . . . . . . . . . . 89

Color Reconnection . . . . . . . . . . . . . . . . . 90

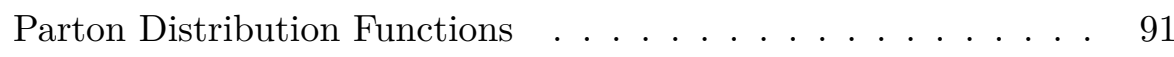

Parton Showering. . . . . . . . . . . . . . . . . . . 92 
5.4.2. Efficiency Scale Factor Uncertainty . . . . . . . . . . . . . . 93

b-tagging Scale Factor . . . . . . . . . . . . . . . 93

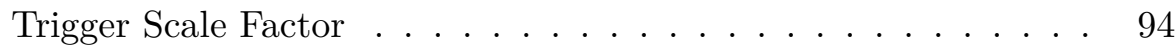

$\tau$ Identification Scale Factor . . . . . . . . . . . . . . . . 94

5.4.3. Background Uncertainty . . . . . . . . . . . . . . . . . 94

$W+$ Heavy Flavor K-factor . . . . . . . . . . . . . . 94

QCD Multijets fraction . . . . . . . . . . . . . 95

5.4.4. Luminosity Uncertainty . . . . . . . . . . . . . . . . . . . . 99

5.5. $\sigma_{t \bar{t}}$ Result with Systematic Uncertainty . . . . . . . . . . . . . . . . 99

6. Top Mass Analysis Method . . . . . . . . . . . . . . . . . . . . . 100

6.1. $\nu$ Scan Method for $\tau$ Lepton Reconstruction . . . . . . . . . . . . . . . . 102

6.2. Likelihood Function . . . . . . . . . . . . . . . . . . . 106

6.3. Event Probability Calculation . . . . . . . . . . . . . . . . . . . . 110

6.3.1. Signal Probability Calculation . . . . . . . . . . . . . . . 114

Integrating the Signal Probability . . . . . . . . . . . 116

$t \bar{t}$ Pair Production Cross Section Top Mass Dependence . . . . . 120

Mean Acceptance . . . . . . . . . . . . . . . . . . . 123

6.3.2. Background Probability Calculation . . . . . . . . . . . . 123

6.3.3. Relative Normalization . . . . . . . . . . . . . . . . . . 126

6.4. Parton-Jet Transfer Functions . . . . . . . . . . . . . . . . . . . . 128

6.4.1. Energy . . . . . . . . . . . . . . . . . 130

$6.4 .2 . \quad$ Angle . . . . . . . . . . . . . . . . . . . . 131

6.5. Maximization and Fit of the Likelihood Function . . . . . . . . . . . . . 141

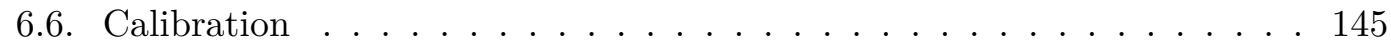

6.7. Expected Uncertainty . . . . . . . . . . . . . . . . . . . 150

6.8. Measurement of $M_{t o p} \ldots \ldots \ldots \ldots \ldots$. . . . . . . . . . 150

7. Systematic Uncertainties for $M_{t o p} \ldots \ldots \ldots \ldots \ldots \ldots \ldots$

7.1. Introduction . . . . . . . . . . . . . . . . . . . . 155 


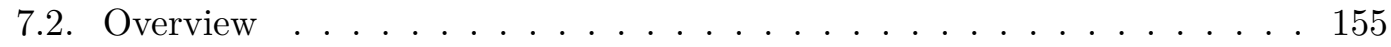

7.3. Jet Energy Scale . . . . . . . . . . . . . . . . . 156

7.4. MC Generator . . . . . . . . . . . . . . . . . . . . . . 157

7.5. Initial and Final State Radiation . . . . . . . . . . . . . . . 159

7.6. Parton Distribution Functions . . . . . . . . . . . . . . . . . . . . 160

7.7. Fraction of $g g \rightarrow t \bar{t}$ Produced Events . . . . . . . . . . . . . 161

7.8. Color Reconnection. . . . . . . . . . . . . . . . . . . . . . . . . . 161

7.9. Background Fraction . . . . . . . . . . . . . . . . . . 164

7.10. MC Statistics . . . . . . . . . . . . . . . . . . . 165

7.11. B Jet Energy Scale . . . . . . . . . . . . . . . . . . . . . 165

7.12. Lepton Energy Scale $\ldots \ldots \ldots$

7.13. Pile-up. . . . . . . . . . . . . . . . . . . . . . . . 169

7.14. $M_{\text {top }}$ Measurement Calibration $\ldots \ldots \ldots \ldots \ldots \ldots$

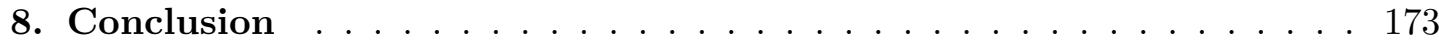

Appendix A. Measurement from a Negative Log Likelihood Function. 175

Appendix B. Smeared Electron Test . . . . . . . . . . . . . . . . 177

Appendix C. Kinematic Event Solution . . . . . . . . . . . . . . . 180

C.1. Hadronic Side . . . . . . . . . . . . . . . . . . . . . . . . . 180

C.2. Leptonic Side . . . . . . . . . . . . . . . . . . . . . . 182

Appendix D. Validating Transfer Function Fits on New Selection . . . 189

Appendix E. Parton Showering Systematic on $\sigma_{t \bar{t}} \ldots \ldots \ldots \ldots$

Appendix F. The Bootstrapping Method . . . . . . . . . . . . . 199 


\section{List of Tables}

1.1. Branching fractions of $\tau$ lepton decay modes . . . . . . . . . . . . . . . 16

2.1. Details of the CDF calorimeter systems . . . . . . . . . . . . . 32

3.1. Tau identification requirements . . . . . . . . . . . . . . . . . . 44

3.2. Electron identification requirements . . . . . . . . . . . . . . 52

3.3. Muon identification requirements . . . . . . . . . . . . . 53

4.1. Cross sections and acceptances used for background estimate . . . . . . 71

4.2. Summary of data/MC scale factors used for background estimate . . . . 72

4.3. Heavy flavor fractions and tagging efficiencies . . . . . . . . . . . 76

4.4. Signal and background contribution estimate . . . . . . . . . . . . . 79

5.1. Details for the calculation of acceptance for $t \bar{t} \rightarrow \tau+$ jets events . . . 86

5.2. Systematic uncertainties on $\sigma_{t \vec{t}} \ldots \ldots \ldots \ldots \ldots$

5.3. QCD multijet fraction for the considered reweighting schemes . . . . . . 96

7.1. Total systematic uncertainties on $M_{\text {top }}$ for the $\tau+$ jets channel. . . . . . 157

7.2. Summary of the JES systematic uncertainty on $M_{t o p} \ldots \ldots \ldots$. . . . 158

7.3. Evaluation of the MC generator systematic uncertainty on $M_{t o p}$. . . . . 159

7.4. Evaluation of the ISR/FSR systematic uncertainty on $M_{t o p} \ldots$. . . . . 160

7.5. Evaluation of the first part of the PDF systematic uncertainty on $M_{t o p}$. 162

7.6. Evaluation of the total PDF systematic uncertainty on $M_{t o p}$. . . . . . . 163

7.7. Evaluation of the $g g$ fusion systematic uncertainty on $M_{t o p} \ldots \ldots$. . . 163

7.8. Evaluation of the color reconnection systematic uncertainty on $M_{t o p}$. . 164

7.9. Evaluation of the background fraction systematic uncertainty on $M_{t o p}$. 165

7.10. Evaluation of the b-jet energy scale systematic uncertainty on $M_{t o p}$. . . 167

7.11. Evaluation of the $\tau$ energy scale systematic uncertainty on $M_{t o p} \ldots \ldots$. 168

7.12. Evaluation of the pileup systematic uncertainty on $M_{t o p} \ldots \ldots$. . . . 171 
7.13. Evaluation of the calibration systematic uncertainty on $M_{t o p}$. . . . . . 172

B.1. Results of smeared electron test . . . . . . . . . . . . . . . . . . . 179

E.1. Comparison of PyTHIA and HERWIG $\tau+$ jets acceptance and $\sigma_{t \bar{t}}$ before and after reweighting . . . . . . . . . . . . . . . . . 198 


\section{List of Figures}

1.1. The particles of the Standard Model . . . . . . . . . . . . . . . . . . 3

1.2. Higgs boson mass prediction based on $\chi^{2}$ fits to electroweak measurements 8

1.3. Leading order Feynman diagrams for $t \bar{t}$ pair production . . . . . . . . . 11

1.4. Theoretical $t \bar{t}$ production cross section versus the top quark mass . . . . 12

1.5. Leading order Feynman diagram for $q \bar{q} \rightarrow t \bar{t} \rightarrow \tau+$ jets $\ldots . . .13$

1.6. Feynman diagrams for top and Higgs loop corrections to $W$ mass . . . . 13

1.7. Top quark mass vs $W$ boson mass which constrains the Higgs boson mass 14

1.8. Feynman Diagram for the decay of a $\tau$ lepton . . . . . . . . . . . . . 16

2.1. Integrated luminosity delivered by the Tevatron . . . . . . . . . . . . 22

2.2. The Fermilab accelerator chain . . . . . . . . . . . . . . . . 23

2.3. Elevation view of the CDF detector. . . . . . . . . . . . . . 24

2.4. Bulkhead view of the SVX module . . . . . . . . . . . . . . . 26

2.5. Side view of CDF tracking region . . . . . . . . . . . . 27

2.6. CDF side view showing tracking region and calorimeters . . . . . . . . . 33

2.7. CDF detector diagrams . . . . . . . . . . . . . . . . 35

3.1. Diagram of $\tau$ reconstruction cone algorithm . . . . . . . . . . . . . . 43

3.2. Fractional uncertainties of the jet energy scale corrections . . . . . . . . 48

3.3. Diagrams of b-tagging secondary vertices . . . . . . . . . . . . 50

3.4. b-tagging efficiency for $b$ jets from top quark decays . . . . . . . . . . 50

3.5. CDF detector signatures for common particles. . . . . . . . . . . . . 54

4.1. QCD multijet model validation . . . . . . . . . . . . . . . 58

4.2. Example structure of a MultiLayerPerceptron neural network . . . . . . 60

4.3. Dalitz variables for $t \bar{t}$ and QCD multijet events . . . . . . . . . . . . . 62

4.4. Neural network input parameter distributions part 1 . . . . . . . 63 
4.5. Neural network input parameter distributions part 2 . . . . . . . . 64

4.6. Training the neural network . . . . . . . . . . . . . . . . 65

4.7. Output of the neural network . . . . . . . . . . . . . . . 66

4.8. Optimizing neural network selection requirement . . . . . . . . . . 68

4.9. $t \bar{t}$ acceptance and sample purity in relation to NN selection . . . . . . . 69

4.10. Fit of the QCD multijet shape to the NN output before b-tagging . . . 73

4.11. Fit of the QCD multijet shape to the NN output after b-tagging . . . . 78

4.12. Distribution of jet $P_{T}$ 's compared in data and expectation . . . . . . . 80

4.13. Kinematic variable distributions compared in data and expectation . . . 81

4.14. $\tau$ variables compared in data and expectation . . . . . . . . . . . . 82

5.1. Negative $\log$ likelihood function with fit for $\sigma_{t \bar{t}}$ measurement . . . . . 87

5.2. NN output shapes for QCD multijet uncertainty evaluation . . . . . . . 97

5.3. Reweighted QCD multijet NN output compared to data . . . . . . . . . 98

6.1. Comparison of $\phi$ and $\theta$ between the hadronic $\tau$ and the $\nu_{\tau}$. . . . . . . 103

6.2. Comparison of the $\phi$ angle of the $E_{\mathrm{T}}$ and the $\nu_{W} \ldots \ldots$. . . . . . 104

6.3. Missing $E_{T}$ resolution for the $\nu$ scan method . . . . . . . . . . . 106

6.4. Resolution of the $\nu_{\tau}$ angles from the $\nu$ scan method . . . . . . . . . . 107

6.5. Energy resolution of the $\tau$ lepton after the $\nu$ scan method . . . . . . . . 108

6.6. Distributions of $t \bar{t}$ system $P_{X}$ and $P_{Y} \ldots \ldots \ldots \ldots$. . . . . . . 112

6.7. Negative $\log$ of signal probability in data and MC . . . . . . . . . . 121

6.8. Invariant mass distributions taken from the Matrix Element jet-parton assignment . . . . . . . . . . . . . . . . . . . . 122

6.9. $t \bar{t}$ pair production cross section versus $M_{t o p} \ldots \ldots \ldots \ldots$

6.10. Acceptance of $t \bar{t} \rightarrow \tau+$ jets versus $M_{t o p} \ldots \ldots \ldots \ldots$. . . . . 124

6.11. Fractional variation of the background probability versus the number of iterations . . . . . . . . . . . . . . . 126

6.12. Negative $\log$ the background probability in data and MC $\ldots \ldots$. . . 127

6.13. Uncertainty on $M_{t o p}$ versus $A_{b k g d} \ldots \ldots \ldots \ldots$. . . . . . . 128

6.14. Energy resolution of jets from light quarks . . . . . . . . . . . . . . 132 
6.15. Energy resolution of jets from $b$ quarks $\ldots \ldots \ldots \ldots$

6.16. Resolution of the angles of the two jets from $W$ decay $\ldots . . . .135$

6.17. Distribution of $M_{W}^{2}$ as reconstructed from the daughter particles . . . . 136

6.18. Resolution of the angle between the two jets from the $W$ decay $\ldots . .137$

6.19. Distribution of the angular TF for $\alpha_{12} \ldots \ldots$. . . . . . . . . . 139

6.20. Distribution of the angle between the two light quark jets before and after application of the angular TF . . . . . . . . . . . . . . . 140

6.21. Resolution of the angle between the hadronic side $W$ and $b$ quark . . . . 142

6.22. Distribution of the angular TF for angle between the hadronic side $W$ and $b$ quark $\ldots \ldots \ldots \ldots \ldots$. . . . . . . . . . . . . . . 143

6.23. Distributions of $M_{t o p}^{2}$ and $M_{W}^{2}$ after applying angular TF's . . . . . . . 144

6.24. Output $M_{t o p}$ versus input $M_{t o p}$ before calibration . . . . . . . . . . 147

6.25. Output $M_{t o p}$ versus input $M_{t o p}$ after calibration . . . . . . . . . . . 148

6.26. Pull widths from $M_{\text {top }}$ measurement before applying the uncertainty calibration . . . . . . . . . . . . . . . . . . . 149

6.27. Residual, Pull, and Pull Widths of measurement versus $M_{t o p}$ after applying all calibrations . . . . . . . . . . . . . . . 151

6.28. Expected statistical uncertainty on $M_{t o p}$ measurement . . . . . . . . . . 152

6.29. Negative $\log$ likelihood with fit for $M_{t o p}$ measurement . . . . . . . . 153

6.30. Expected uncertainty distribution from ensemble of PE's. . . . . . . . . 154

7.1. Evaluation of the uncertainty on the $\tau$ energy scale at CDF . . . . . . 168

7.2. Number of primary vertices in data and $t \bar{t} \mathrm{MC}$. . . . . . . . . . . . 170

B.1. Smearing electron energy resolution to match that observed in $\tau^{\prime}$ s $\ldots 178$

D.1. Comparing the Energy TF with new event selection . . . . . . . . . . . 190

D.2. Comparing the Angular TF with new event selection . . . . . . . . . . . 191

E.1. Distribution of particles resulting in a reconstructed $\tau$. . . . . . . 193

E.2. N-1 distributions for $\tau$ visible mass and number of tracks in isolation annulus . . . . . . . . . . . . . . . . . . . . 194

E.3. N-1 distributions for $\tau$ calorimeter isolation and signal cone tracks . . . 195 
E.4. Number of $\pi^{0}$ 's and visible mass for reconstructed $\tau$ 's matched to generated $\tau^{\prime} s \ldots \ldots \ldots \ldots \ldots \ldots$. . . . . . . . . . . . . . . . . . .

E.5. Distributions of $\tau$ variables for $\tau$ 's from light quarks as observed in Pythia, Herwig, and data . . . . . . . . . . . . . . . . . 198

F.1. Diagram of bootstrapping method . . . . . . . . . . . . . . . 201

F.2. Gaussian toy model illustrating bootstrapping method . . . . . . . . . . 202 


\section{Chapter 1}

\section{Theory and Motivation}

In this thesis, we present the first exclusive observation of the $t \bar{t} \rightarrow$ hadronic $\tau+$ jets decay channel. Using these events (referred to in this thesis as $\tau+$ jets), we measure the $t \bar{t}$ pair production cross section $\left(\sigma_{t \bar{t}}\right)$ and the top quark mass $\left(M_{t o p}\right)$. The $t \bar{t}$ pair production cross section in the $\tau+$ jets decay channel has not been measured at CDF, and the top quark mass measurement presented here is the first measurement ever in this decay channel. To make these measurements, we introduce an artificial neural network to discriminate signal and background events, and we develop a new method for reconstructing the neutrino from the $\tau$ decay based on a collinear approximation (described in Sec 6.1). D $\varnothing$ has measured the $t \bar{t}$ cross section multiplied by branching ratio of $t \bar{t}$ decaying to hadronic $\tau+$ jets in $1 \mathrm{fb}^{-1}$ of data. From this measurement, they extract a $t \bar{t}$ pair production cross section of $6.9 \pm 2.2$ pb assuming a top quark mass of $170.0 \mathrm{GeV}[1$. The various decay modes of top quark pairs are discussed in Sec. 1.3 but since nearly $10 \%$ of all $t \bar{t}$ pairs decay via the hadronic $\tau+$ jets decay mode, this is the largest section of the $t \bar{t}$ decay modes to never be directly explored. We check for agreement with previous measurements in other decay channels as a test of lepton universality. Meanwhile, any discrepancy may be a sign of new physics such as a top quark decaying to a charged Higgs boson and $b$ quark rather than a $W$ boson and a $b$ quark (as described in [2]). Finally, these measurements demonstrate that complicated physics, such as a so called "Matrix Element" measurement of the top quark mass like the one presented in Chap. 6 can be performed in high jet multiplicity environments. This last point is especially noteworthy now as the Large Hadron Collider (LHC) at CERN is exploring a new energy frontier in particle physics colliding protons at a centerof-mass energy of $7 \mathrm{TeV}$. At the LHC, the multijets background from QCD processes 
dominates many signal processes at a level beyond that at the Tevatron. However, the results presented here verify that we can supress these backgrounds and complete complicated analyses in such an enviroment.

This chapter gives a brief overview of the Standard Model, the theoretical model which describes the elementary particles and the interactions between them. Special consideration is given to the top quark and $\tau$ lepton as they are the main focus of the analysis presented in this thesis. It should also be noted that throughout this thesis, we use notation in which $c=1$.

\subsection{The Standard Model}

The Standard Model (SM) is a theoretical construct of the known fundamental particles and the interactions of the Strong, Weak, and Electromagnetic forces, 3 of the 4 known forces in nature. It is an incomplete theory in that it does not have a formulation for the fourth known force, Gravity. The SM does an excellent job of describing the interactions between particles since the gravitational force is too weak to have observable effects at the subatomic level. The model contains 12 spin $\frac{1}{2}$ fermions and their 12 antiparticle partners which are the constituents of matter. It also describes the 3 included forces through the Gauge group $S U(3) \times S U(2) \times U(1)$. These forces are mediated by 4 spin 1 bosons. The strong force is represented by the $S U(3)$ group and acts between particles with color charge. Its interactions are mediated by the gluon $(\mathrm{g})$. The $S U(2) \times U(1)$ group represents the Electroweak force, a unification of the Electromagnetic and Weak forces. The Weak force acts on all fermions and is mediated by the massive $W$ and $Z$ bosons. Finally, the Electromagnetic force acts between electrically charges particles and is mediated by the massless photon $(\gamma)$. Additionally, the Standard Model includes the Higgs mechanism which breaks the Electroweak symmetry. This mechanism predicts the existence of the Higgs boson which interacts with the elementary fermions and the $W$ and $Z$ bosons, as well as itself, to give them mass. The Higgs boson is the only particle in the Standard Model which has yet to be observed. The confirmed particles of the Standard Model organized into the 3 generations of fermions and the force mediating bosons are shown in Fig. 1.1 along with each particle's mass, electric 
charge, and spin.

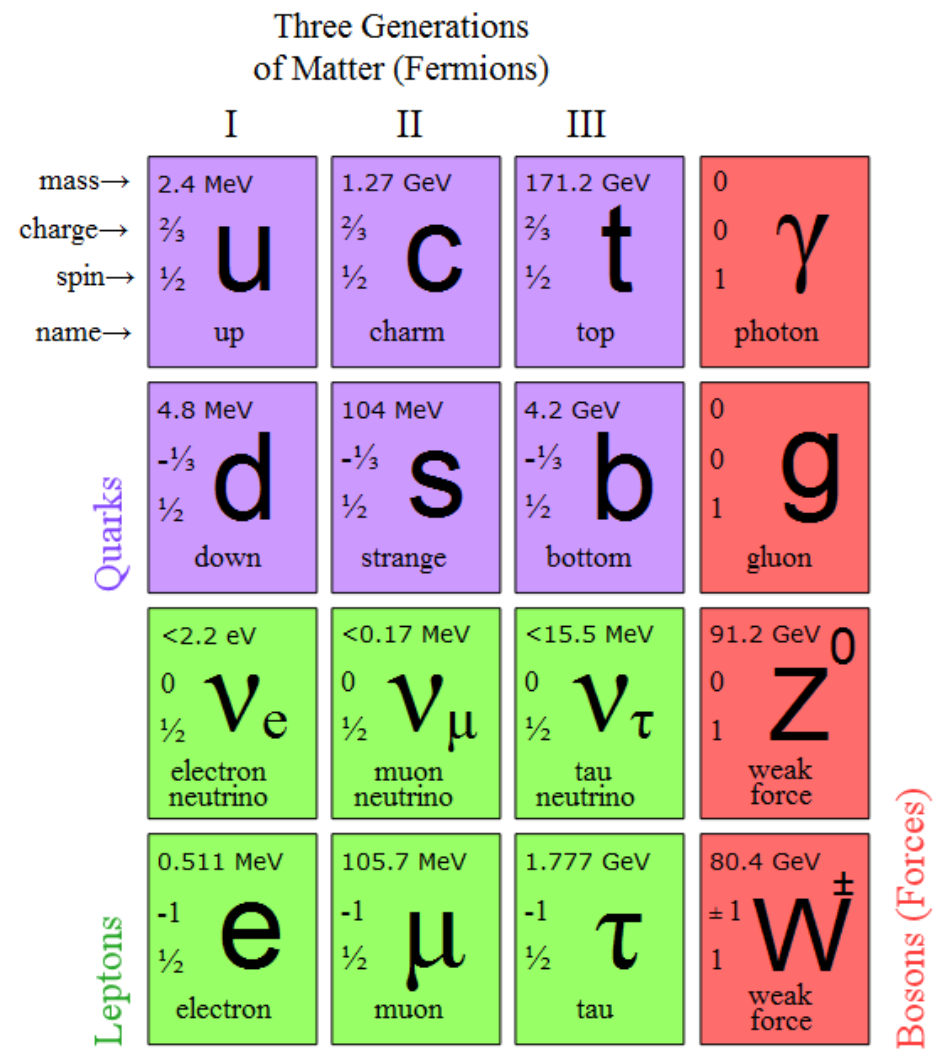

Figure 1.1: Representation of 12 fermion and 4 force carrying bosons of the Standard Model [3]. Note, the Higgs boson is not included.

The 12 fermions are divided into 6 quarks and 6 leptons. The main difference between these particles is that leptons, unlike quarks, do not carry any color charge and thus do not interact via the strong force. These 12 particles are grouped into 3 generations of 4 particles each: an "up" type quark with electric charge $\frac{2}{3} \mathrm{e}$, a "down" type quark with electric charge $-\frac{1}{3} \mathrm{e}$, an electric charge -1e lepton, and an electric charge neutral lepton called a neutrino. The first family of particles, composed of the up $(u)$ and down $(d)$ quarks, the electron $(e)$, and electron neutrino $\left(\nu_{e}\right)$, are stable particles which do not decay. The second generation is composed of the charm $(c)$ and strange $(s)$ quarks, the muon $(\mu)$, and the muon neutrino $\left(\nu_{\mu}\right)$. The final generation is composed of the top $(t)$ and bottom $(b)$ quarks, the tau $(\tau)$, and the tau neutrino $\left(\nu_{\tau}\right)$. It is the particles of this third generation in which we are mainly interested for this analysis. 
The particles in each generation are heavier than their counterpart from the previous generation with the top quark being by far the most massive known elementary particle. Until the last decade, the neutrinos were believed to be massless; however, experimental results in the early 2000's have shown that neutrinos can oscillate between flavors. This phenomenon requires that the neutrinos carry some mass [4]. The exact value of the three $\nu$ masses is known to be extremely small but has never been experimentally measured due to the difficulty in observing neutrinos as they only interact via the weak force and have very low interaction cross sections. It should be noted that although the fermion masses in the SM are results of the fermion's interaction with the Higgs boson, the exact mass of each of the fermions is not predicted. The fermion masses are necessary input parameters in the model and must be determined via experimental measurement.

The strong force is represented in the SM via the $\mathrm{SU}(3)$ non-Abelian gauge group. This gauge symmetry leads to the field theory of Quantum Chromodynamics (QCD) which describes the interactions of the strong force. The strong force acts between particles with color charge. Each quark carries a color charge (red, blue, or green), and each gluon carries a color and anti-color charge. As a result, gluons can couple to other gluons as well as quarks. There are 8 different color states of gluons. Although one would initially expect 9 , the color singlet state of the gluon, such as $(r \bar{r}+b \bar{b}+g \bar{g}) / \sqrt{3}$, is color neutral and would not interact with color charged particles. Such a color neutral gluon would only interact with other color neutral states, and since we do not see evidence of strong force interactions between color neutral particles, this $9^{\text {th }}$ gluon must not exist [5]. Because of the strong force, quarks are found bound within color neutral hadrons. These hadrons are referred to as baryons when they are composed of 3 quarks each carrying a different one of the three color charges and mesons when they are composed of a quark anti-quark pair where the anti-quark carries the anticolor charge of the quark (as in a red anti-red pair). Unlike the other forces, the strong force does not diminish as the distance between color charged particles increases. This leads to the principle of confinement. Confinement simply states that a bare quark (not bound in a color neutral hadron) will not exist in nature. This is because as the distance between 
two quarks increases, the color force continues to grow and the energy in the color field between them increases. Eventually, a point is reached that there is so much energy stored in the color field that it pulls a quark anti-quark pair out of the vacuum. These new quarks interact with the original quarks to form new color neutral hadrons. This makes it impossible to separate a quark from a color neutral hadron [6]. Additionally, the strong interaction coupling constant, $g_{s}$, decreases logarithmically as a function of energy. This is referred to as asymptotic freedom. As a result, the cross section for deep inelastic scattering processes in high energy environments such as $q \bar{q}$ annihilation to a $t \bar{t}$ pair at the Tevatron can be calculated perturbatively. However, at low energies, the strong interaction coupling becomes very strong, and the QCD processes such as multijet production or the hadronization of a bare quark are impossible to calculate via perturbation theory [7].

At very high energies, the electromagnetic and weak forces are unified into a single interaction called the electroweak force. This interaction is represented by the $S U(2) \times$ $U(1)$ gauge group. At energies around $1 \mathrm{TeV}$, this gauge symmetry is spontaneously broken which gives rise to the separate weak and electromagnetic interactions. The electromagnetic interaction acts between particles which carry electric charge and is carried by the massless $\gamma$. The weak force is mediated by three massive bosons, the $W^{+}, W^{-}$, and the electrically neutral $Z$. The masses of the $W$ and $Z$ bosons have been measured to be $80.40 \pm 0.02 \mathrm{GeV}$ and $91.188 \pm 0.002 \mathrm{GeV}$, respectively [3]. The weak force acts on all fermions and is the only force by which more massive quarks can decay into lighter quarks. The reasoning for this limitation is that flavor changing neutral currents are highly suppressed in the SM. The explanation for this begins with Cabbibo who postulated that the Weak force couples $u$ quarks to a mixed eigenstate of $d$ and $s$ quarks known as $d^{\prime}$ [5]. At this time, only the first two generations of fermions were known with the absence of the $c$ quark. Glashow, Iliopoulos, and Maiani (GIM) predicted the existence of the $c$ quark to explain the lack of any experimental observation of flavor changing neutral currents and a desire to symmetrize the first and second generations of matter. They expanded Cabbibo's work by coupling $u$ quarks to the eigenstate $d$ ' and $c$ quarks to a similar mixed eigenstate of $d$ and $s$ quarks called 
$s^{\prime}[5]$ [6]. These eigenstates are defined by a unitary matrix, and as a result, the neutral terms which couple $d$ and $s$ quarks are canceled out by equal but oppositely signed amplitudes. This argument was extended to the current version of the SM with 3 generations of fermions by Kobayashi and Maskawa to describe the mixing between quarks of all three flavors [5] 6] 8]. Consider the possible decay modes of the "up" type top quark. In order to conserve electric charge, the top quark must decay either neutrally via a $Z$ or $\gamma$ to a different flavor "up" type quark or via a charged $W^{-}$to any flavor "down" type quark. Due to the suppression of the first option, the only possible decay channel for a top quark is to a $W$ boson and the "down" type eigenstate $b$ ' made up of a superposition of $d, s$, and $b$ quarks. The transformation between the mass eigenstate quarks $q$ and the superposition eigenstates $q$ ' involved in the coupling is described by the Cabibbo-Kobayashi-Maskawa (CKM) matrix:

$$
\left(\begin{array}{ccc}
V_{u d} & V_{u s} & V_{u b} \\
V_{c d} & V_{c s} & V_{c b} \\
V_{t d} & V_{t s} & V_{t b}
\end{array}\right)
$$

where $V_{i j}$ describes the coupling between an "up" type quark of flavor i with a "down" type quark of flavor $\mathrm{j}$. The CKM matrix is required to be unitary, and each of the terms is complex. This matrix is applied to the mass eigenstates $q$ to generate the superposed coupling eigenstates $q$ ' as:

$$
\left[\begin{array}{l}
\mid d^{\prime}> \\
\mid s^{\prime}> \\
\mid b^{\prime}>
\end{array}\right]=\left(\begin{array}{ccc}
V_{u d} & V_{u s} & V_{u b} \\
V_{c d} & V_{c s} & V_{c b} \\
V_{t d} & V_{t s} & V_{t b}
\end{array}\right) \cdot\left[\begin{array}{l}
\mid d> \\
\mid s> \\
\mid b>
\end{array}\right]
$$

The quantity $\left|V_{i j}\right|^{2}$ gives the probability of transitioning from a quark of type $\mathrm{i}$ to a quark of type $\mathrm{j}$ via an interaction with a $W$ boson. The amplitudes of the CKM matrix elements are experimentally measured to be $[9]$ :

$$
\left(\begin{array}{ccc}
\left|V_{u d}\right| & \left|V_{u s}\right| & \left|V_{u b}\right| \\
\left|V_{c d}\right| & \left|V_{c s}\right| & \left|V_{c b}\right| \\
\left|V_{t d}\right| & \left|V_{t s}\right| & \left|V_{t b}\right|
\end{array}\right)=\left(\begin{array}{ccc}
0.97428 \pm 0.00015 & 0.2253 \pm 0.0007 & 0.00347_{-0.00012}^{+0.00016} \\
0.2252 \pm 0.0007 & 0.97345_{-0.00016}^{+0.00015} & 0.0410_{-0.0007}^{+0.0011} \\
0.00862_{-0.00020}^{+0.00026} & 0.0403_{-0.0007}^{+0.0011} & 0.999152_{-0.000045}^{+0.000030}
\end{array}\right)
$$


It is worthwhile to note that Eqn. 1.3 shows that while the flavor mixing between the first two generations of quarks is fairly significant, the mixing between the third flavor of quarks, the $t$ and $b$ quarks, and the first two flavors is very small. Thus, we find that the top quark decays almost exclusively as $t \rightarrow W^{-} b$.

To this point, the description of the SM does not account for the masses of the fermions. Perhaps more concerning though is the lack of an explanation for the large masses of the $W$ and $Z$ bosons. There is no way to add mass terms for these bosons to the SM Lagrangian without breaking the gauge symmetry of the theory. This problem is solved in the SM via the Higgs mechanism [7]. The Higgs mechanism breaks the electroweak symmetry by introducing a scalar Higgs field with a non-zero vacuum expectation value. To match the observation of three massive bosons, the scalar Higgs field must be an $\mathrm{SU}(2)$ doublet of complex fields. This field is mediated by 4 bosons, two electrically charged and two electrically neutral. The two charged bosons and one of the neutral bosons are Goldstone bosons which become the longitudinal polarization of the $W^{ \pm}$and $Z$ bosons thus giving these weak force mediators mass. A single massive spin 0 boson known as the Higgs boson remains. The mass of this boson is given by the equation $m_{H}=\sqrt{2 \lambda} v$ where $\lambda$ is the quartic self coupling term of the Higgs field and $v$ is the vacuum expectation value. The Higgs boson also gives fermions their mass through the interaction between each fermion with the Higgs field described by their Yukawa coupling (a coupling between a Dirac field or fermion and a scalar field such as the Higgs field). These Yukawa couplings are each input parameters for the SM and must be empirically determined. Currently, the Higgs boson is the only SM particle which has yet to be observed. Direct searches at LEP and the Tevatron experiments have rules out the Higgs mass $\left(m_{\text {Higgs }}\right)$ regions $m_{\text {Higgs }}<114 \mathrm{GeV}$ [10] and $156 \mathrm{GeV}<m_{\text {Higgs }}<177 \mathrm{GeV}$ [11]. The Higgs mass can also be indirectly probed via $\chi^{2}$ fits of high precision electroweak measurements which can be seen in Fig. 1.2 . By a conservative estimate, ongoing searches at the Large Hadron Collider at CERN should see evidence of or rule out the existence of the SM Higgs boson within the next few years. 


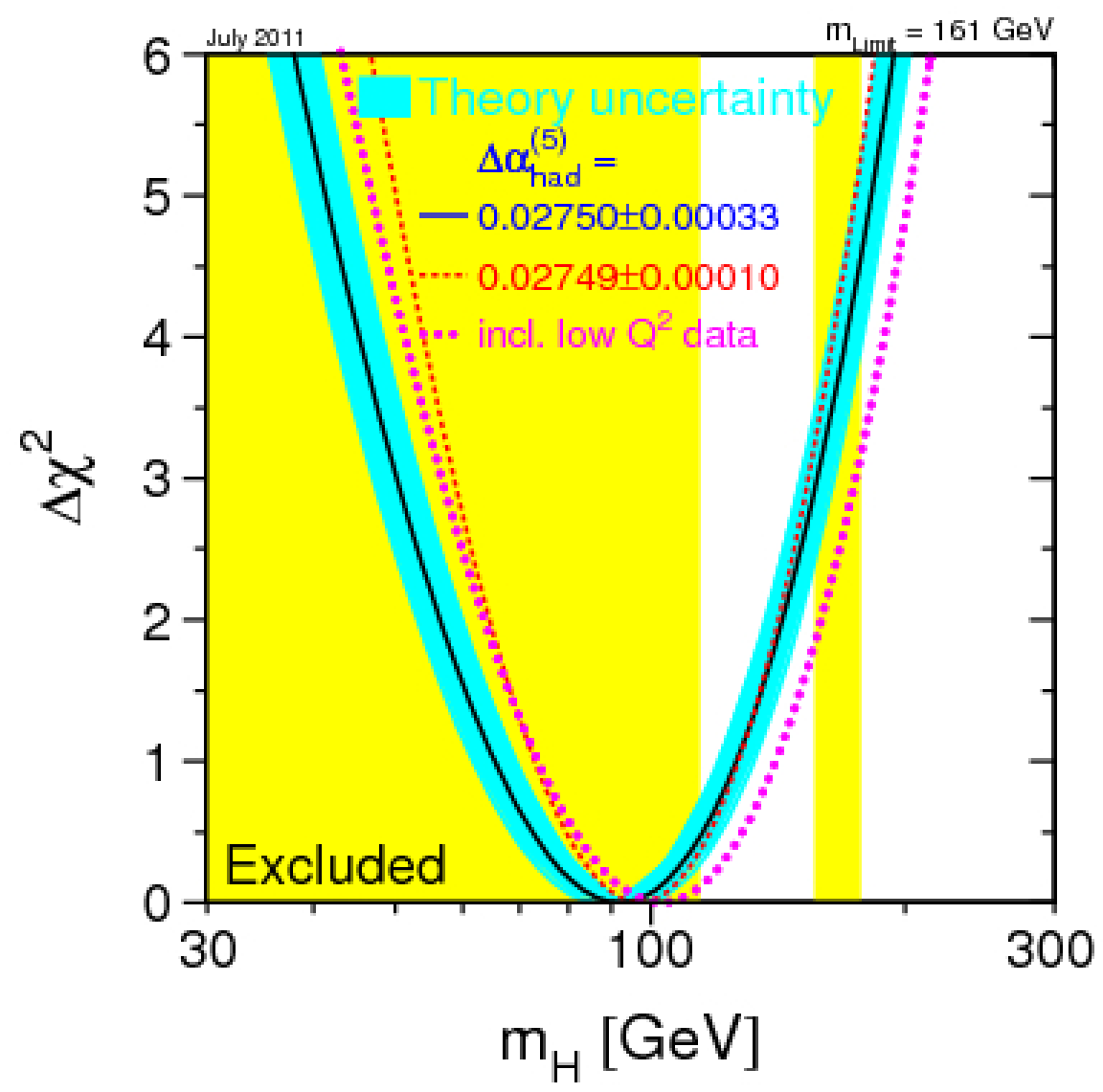

Figure 1.2: $\Delta \chi^{2}$ fits of high precision electroweak measurements made at LEP, SLD, $\mathrm{CDF}$, and D0 as a function of the mass of the SM Higgs boson. The $\chi^{2}$ distribution prefers a Higgs mass of $92_{-26}^{+34} \mathrm{GeV}$ with an upper limit of $161 \mathrm{GeV}$. The yellow bands represents mass regions excluded directly by LEP and the Tevatron experiments [12]. 


\section{$1.2 \quad$ Matrix Elements}

The cross section for any physical process is described by the amplitude of the interactions involved and the phase space available for the process. The amplitude is also know as the matrix element $(M)$ which is an element of the S-matrix describing all possible processes linking an incoming set of particles $\vec{A}$ to an outgoing set $\vec{B}$ including the identity matrix for the case in which the incoming particles fail to interact with each other [7]. For a specific process, the matrix element is graphically represented by a Feynman diagram. It represents all the information involved in the dynamics of the interaction (propagators of intermediate states and couplings between fermions and force carrying bosons) and is independent of the kinematics of the final state particles which are described by the phase space [5]. Because the matrix element describes all the dynamics of a process including the invariant mass of the particles, we can use it to build a probability for $t \bar{t} \rightarrow \tau+$ jets events that uses the maximum amount of information available in each event. The exact method of building these probabilities

and the calculation method of the $t \bar{t}$ production matrix element will be described in Chap. 6

\section{$1.3 \quad$ Top Quark}

The top quark is the "up" type quark in the third generation of fermions. It was discovered at Fermilab in 1995 [13] [14][15]. It is by far the heaviest of all the elementary particles with a mass of $173.2 \pm 0.9 \mathrm{GeV}$ as measured at the Tevatron as of July 2011 with $5.8 \mathrm{fb}^{-1}$ of data [16]. In fact, it dwarfs the next most massive fermion, its third generation "down" type partner the $b$ quark which has a mass of approximately 4 $\mathrm{GeV}$. Because of its high mass, the top quark has an extremely short lifetime on the

order of $10^{-25} \mathrm{~s}$. In this regard, the top quark is unique in that it is the only quark with a lifetime shorter than the time it takes to hadronize (approximately $10^{-24} \mathrm{~s}$ ). The top quark thus is the only quark which can have its properties measured directly from its daughter particles. For this reason, the top quark properties can be measured much more precisely than the properties of any other quark. Additionally, the Yukawa 
coupling of the top quark to the Higgs boson is found to be nearly equal to 1 . This would seem to be an odd coincidence and has led physicists to believe that the top quark may play a special role in the electroweak symmetry breaking.

At the Tevatron at Fermilab top quarks are produced in $p \bar{p}$ collisions with centerof-mass energy $(\sqrt{s})$ equal to $1.96 \mathrm{TeV}$. At this energy scale, the top quark is primarily produced in $t \bar{t}$ pairs via strong interactions either through the annihilation of a $q \bar{q}$ pair of through a process known as $g g$ fusion (see Fig. 1.3). Next-to-leading-order (NLO) predictions of the amplitude for $t \bar{t}$ pair production at the $1.96 \mathrm{TeV}$ energy at the Tevatron predict $15 \pm 5 \%$ of $t \bar{t}$ pairs to come from $g g$ fusion [17] while the rest come from $q \bar{q}$ annihilation. As of August 2009, the $t \bar{t}$ pair production cross section as calculated from $4.6 \mathrm{fb}^{-1}$ of events at the $\mathrm{CDF}$ detector at Fermilab is measured to be $7.5 \pm 0.5 \mathrm{pb}$ assuming a top quark mass of $172.5 \mathrm{GeV}[18]$. This measurement along with theoretical predictions of the $t \bar{t}$ pair production cross section as a function of mass is shown in Fig. 1.4. The top quark can also be produced singly with another quark via the space-like (s-channel) or time-like (t-channel) exchange of a $W$ boson between quarks. For the sake of this analysis, these single top production events are treated as a source of background events. The cross section for single top production summed over the s and t channel production at the Tevatron is measured to be $2.76_{-0.47}^{+0.58} \mathrm{pb}[19]$. Due to the smaller cross section and the different decay signature, single top production events provide a very small contribution to the events selected in this analysis.

As the CKM matrix showed in Eqn. 1.3, the mixing between the third generation quarks and the quarks from the first two generations is very small and the top quark decays almost exclusively to a $W$ boson and $b$ quark. As a result, the produced $t \bar{t}$ pair decays into the 4 particles $W^{-} b W^{+} \bar{b}$. Since the $W$ boson can decay either leptonically to a charged lepton and a neutrino or hadronically to a $q \bar{q}$ pair. We break down the $t \bar{t}$ decay into 3 categories based on how the $W$ bosons decay. If both $W$ bosons decay hadronically, we get a final state with 6 quarks called the all-hadronic $t \bar{t}$ decay. If both $W$ bosons decay leptonically, the final state contains the two $b$ quarks, 2 leptons, and $2 \nu$ 's. These decays are referred to as dileptonic $t \bar{t}$ decays. Finally, we have the case where one $W$ decays leptonically and the other decays hadronically. This gives a final 

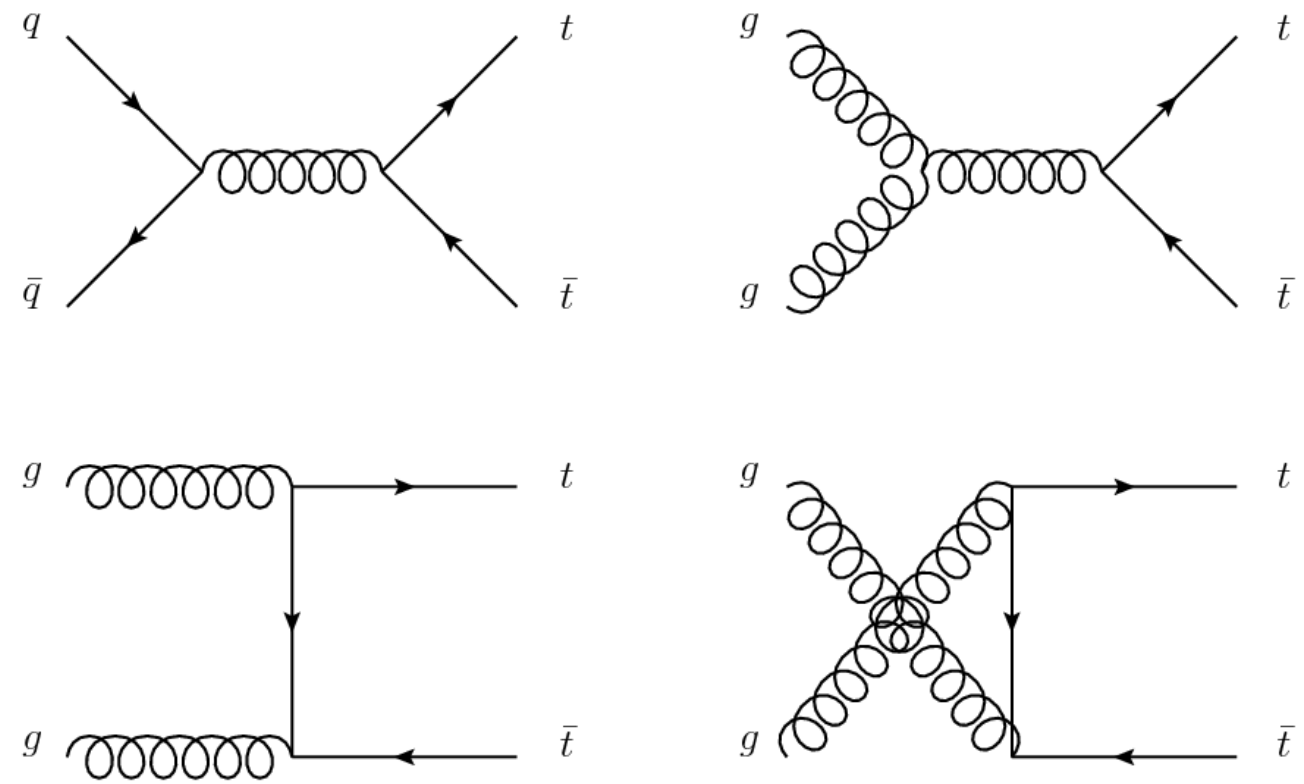

Figure 1.3: Feynman diagrams for $t \bar{t}$ pair production via $q \bar{q}$ annihilation (upper left) and $g g$ fusion.

state with 4 quarks, a single charged lepton, and a $\nu$. These decays are referred to as semi-leptonic or lepton + jets decays. It is the lepton + jets final state, specifically those in which the charged lepton is a $\tau$ lepton, depicted in Fig. 1.5, in which we are interested for this analysis. The leading order Feynman diagram in Fig. 1.5 will be used to compute the $t \bar{t}$ matrix element for the mass measurement in Chap. 6 .

Along with the tests of lepton universality, precision top quark mass measurements are important in the SM because, along with precision $W$ boson mass measurements, they constrain the SM Higgs boson mass. Radiative corrections to the $W$ mass, such as those depicted in the Feynman diagrams in Fig. 1.6 link the top, $W$, and Higgs masses. The Higgs mass is not predicted by the SM, therefore the only way to constrain it is by using precision electroweak measurements which show dependence on the Higgs mass. The most recent Higgs constraint from precision $W$ and top quark masses can be seen in Fig. 1.7. 


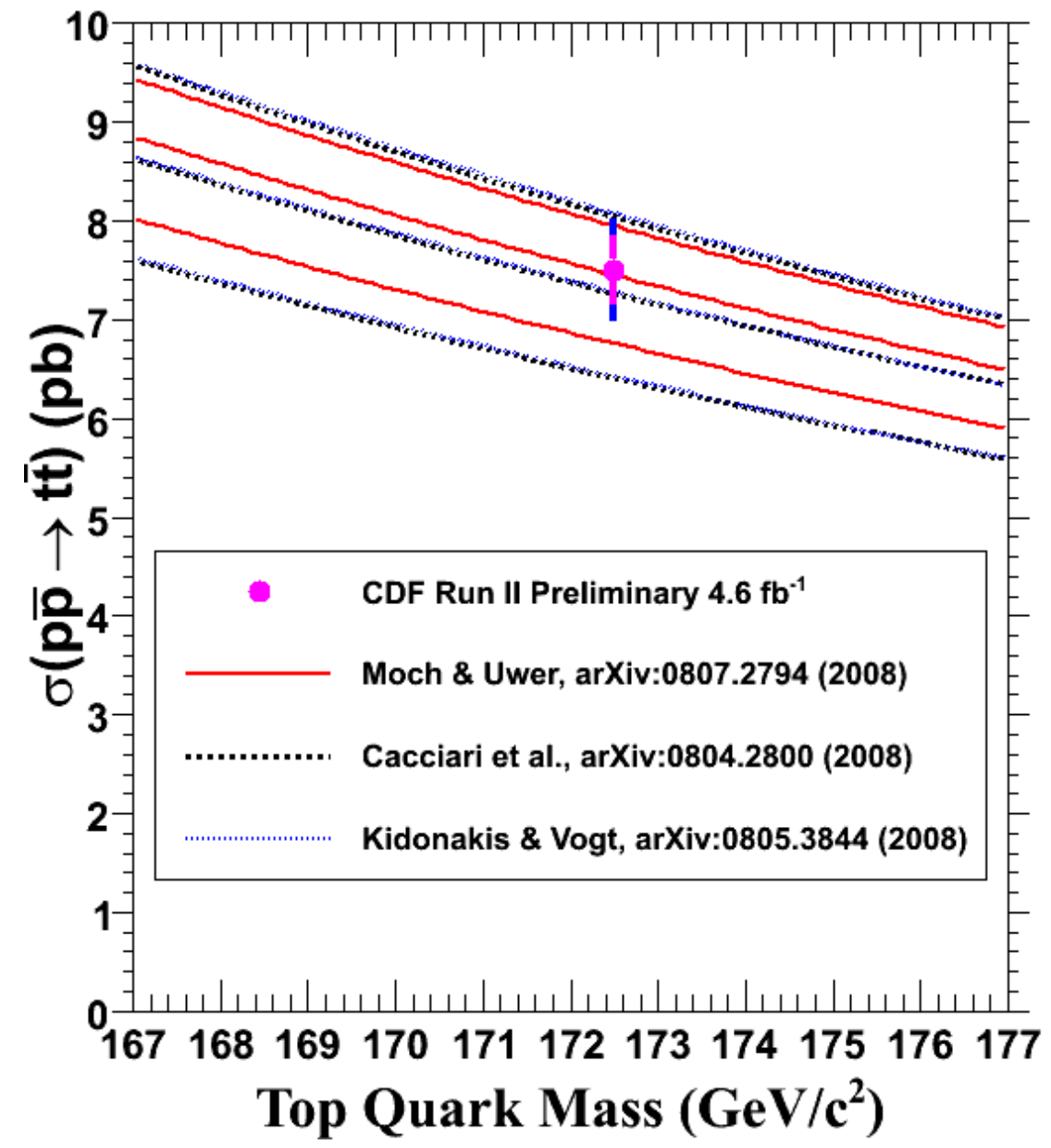

Figure 1.4: Theoretical calculations of top cross section as a function of top mass. The most current CDF combination is also marked [20]. 


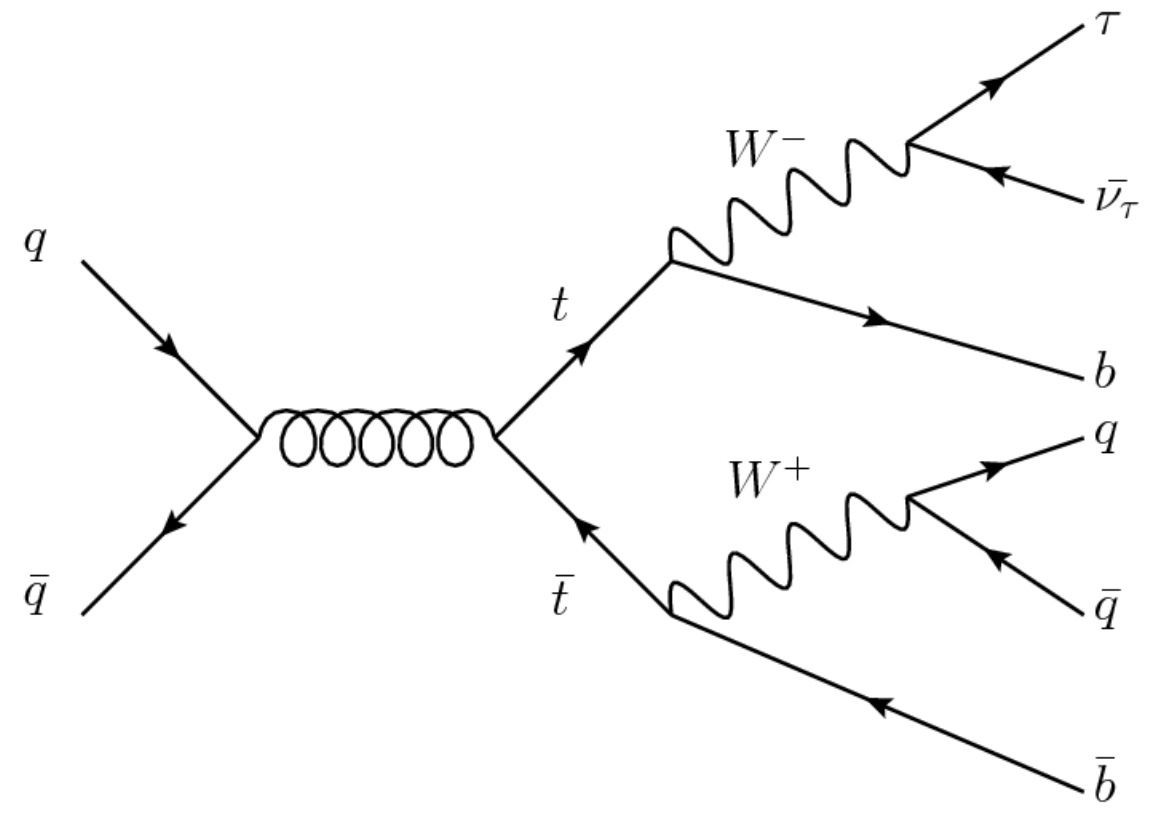

Figure 1.5: Leading order Feynman diagram for pair production of $t \bar{t}$ followed by $t \bar{t}$ decay to the $\tau+$ jets decay channel
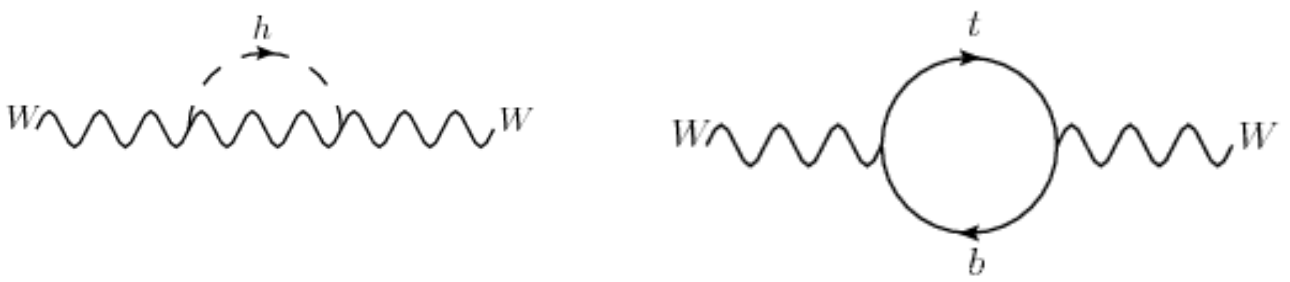

Figure 1.6: Examples of Feynman diagrams of $W$ propagators with radiative loops which give rise to a $W$ mass dependence on the Higgs mass (left) and top quark mass (right). 


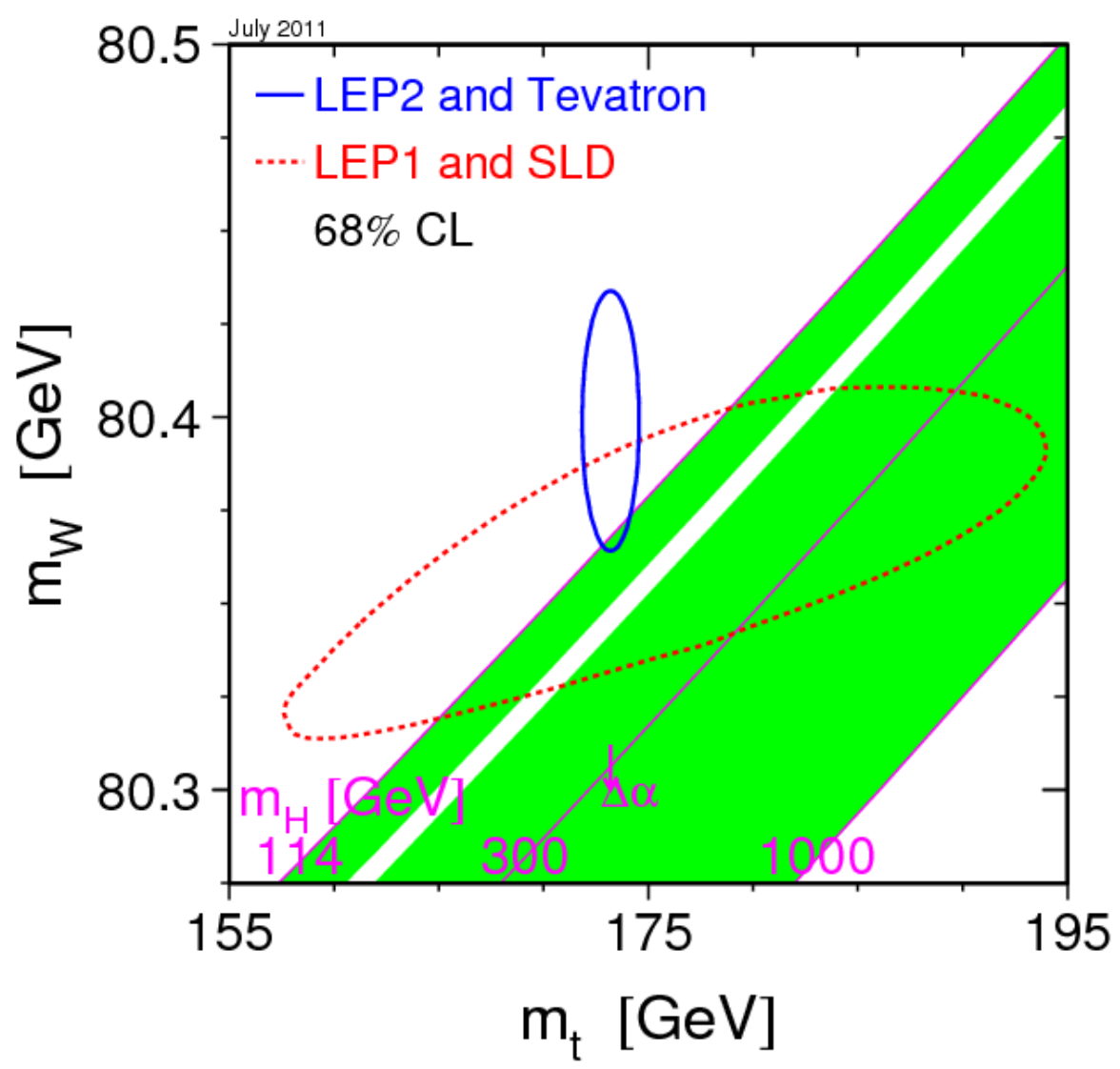

Figure 1.7: Top quark mass vs $W$ mass measurements from LEP, SLD, and the Tevatron experiments. Together, these measurements constrain the SM Higgs boson mass (drawn along diagonal lines). The green shaded regions are Higgs boson masses which are not excluded by LEP and the Tevatron [21]. 


\section{$1.4 \tau$ Leptons}

The $\tau$ lepton is the charged lepton component of the third generation of fermions. In this way, it can be viewed as a heavy electron. It was discovered in the mid 1970's by a collaboration between SLAC and LBL. Researchers found an excess of anomalous events of the form $e^{-}+e^{+} \rightarrow e^{ \pm}+\mu^{\mp}+E_{\mathrm{T}}$. They realized that this process could be explained by the production and decay of a pair of new particles $e^{-}+e^{+} \rightarrow \tau^{-}+\tau^{+} \rightarrow e^{ \pm}+\mu^{\mp}+4 \nu$ [22]. Like the other charged lepton, the $\tau$ interacts via the electromagnetic and weak forces. Just as the top quark is the most massive of the quarks, the $\tau$ lepton is by far the most massive lepton. At a mass of $1.77682 \pm 0.00016 \mathrm{GeV}$ [3] it drawfs the $105.7 \mathrm{MeV}$ muon and $0.511 \mathrm{MeV}$ electron. This high mass means that the $\tau$ has a relatively short lifetime of $2.9 \times 10^{-13} \mathrm{~s}$. The $\tau$ decays via the weak force, and, due to its significantly higher mass, it is the only lepton that can decay to hadrons. The $\tau$ lepton is a good tool to use to search for new physics as its high mass may cause it to be a preferred decay channel for new high mass particles (such as a charged Higgs boson as described in [23]).

We can describe two types of $\tau$ decays: leptonic and hadronic. In all decays, the $\tau$ decays to a virtual $W$ boson and a $\nu_{\tau}$ to conserve the $\tau$ lepton number. In the leptonic decays, the virtual $W$ decays to an electron or muon and the corresponding $\nu$. In hadronic decays, the virtual $W$ decays hadronically producing charged and neutral mesons. See Fig. 1.8 for the Feynman diagram for $\tau$ decays. The branching fraction of each of these decays is governed by the branching fraction of the $W$ boson. As a result, the $\tau$ decays leptonically about $35 \%$ of the time and hadronically the remaining $65 \%$ of the time [3]. The specific breakdown of the $\tau$ decay branching fraction for leptonic and hadronic decay modes are given in Tab. 1.1. As the electron and muon from $\tau$ decay are nearly indistinguishable from electrons and muons from other processes, we chose to focus only on hadronically decaying $\tau$ 's. The specifics of the hadronic $\tau$ decay signature will be discussed in Sec. 3.2.1. 


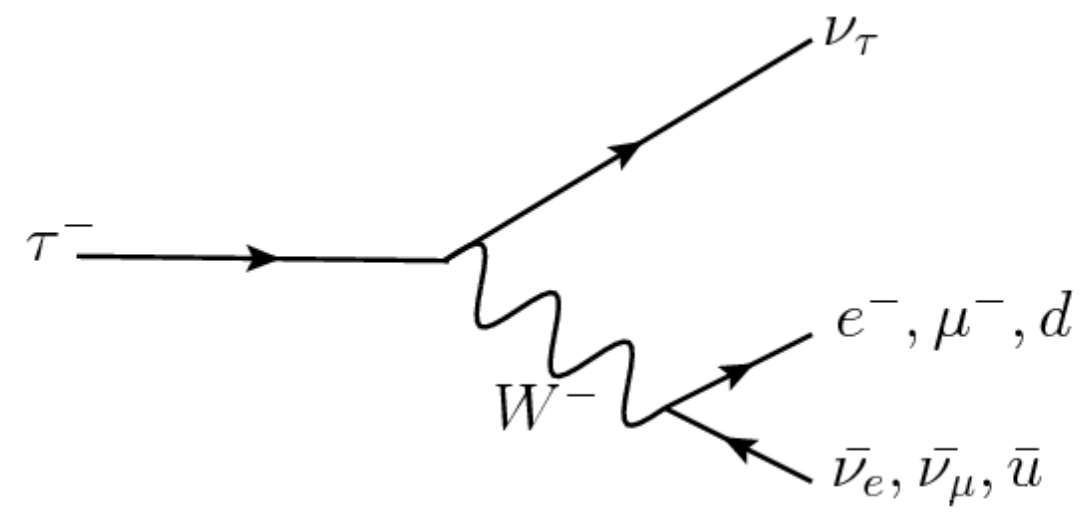

Figure 1.8: Feynman Diagram for the decay of a $\tau$ lepton

\begin{tabular}{|l|c|}
\hline Decay mode & Branching Fraction (\%) \\
\hline$e^{-} \overline{\nu_{e}} \nu_{\tau}$ & $17.82 \pm 0.04$ \\
\hline$\mu^{-} \overline{\nu_{\mu}} \nu_{\tau}$ & $17.39 \pm 0.04$ \\
\hline Hadronic decay & $64.81 \pm 0.12$ \\
\hline \hline \multicolumn{2}{|c|}{ Hadronic modes } \\
\hline 1 prong & $50.15 \pm 0.09$ \\
3 prong & $14.56 \pm 0.07$ \\
5 prong & $0.102 \pm 0.004$ \\
7 prong & $<3.0 \times 10^{-5}$ \\
\hline
\end{tabular}

Table 1.1: A list of possible $\tau^{-}$decay modes with their relative branching fractions [3]. The table also includes the branching fraction for the hadronic modes with a various number of charged particles. Roughly $.1 \%$ of all $\tau$ 's decay hadronically with more than 3 charged particles which is why we select $\tau$ 's with 1 or 3 charged tracks but not 5 or more. 


\subsection{Overview of Chapters}

The rest of this thesis describes the experiment and methods used to measure the $t \bar{t}$ pair production cross section and the top quark mass in $t \bar{t} \rightarrow \tau+$ jets events. Chapter 2 describes the Tevatron which provides $p \bar{p}$ collisions at $\sqrt{s}=1.96 \mathrm{TeV}$ and the CDF detector which we use to observe the decay of particles created in these collisions. Chapter 3 describes how various final state particles appear in the CDF detector as well as how we identify these particles and select $t \bar{t}$ decay events. The backgrounds relevant to this analysis and the method used to estimate their contribution in the data sample are described in Chap. 4. Chapter 5 details the method used to measure the $t \bar{t}$ pair production cross section, gives the resulting measurement, and described the relevant systematic uncertainties on the cross section measurement. The method used to measure the top quark mass cross section is described in Chap. 6 along with the resulting measurement, and the systematic uncertainties for the top quark mass are detailed in Chap. 7. Finally, our conclusions are presented in Chap. 8. 


\section{Chapter 2}

\section{The Tevatron and the CDF Detector}

We examine top quark pairs produced in $1.96 \mathrm{TeV}$ center-of-mass energy $(\sqrt{s}) p \bar{p}$ collisions. The collisions are produced by the Tevatron, a $2 \mathrm{~km}$ in diameter synchrotron located in Batavia, Illinois at Fermilab. A synchrotron is a ring shaped accelerator that uses synchronized electric and magnetic fields to accelerate charged particles. The electric fields boost the particle's energy while the magnetic field is used to turn the particle in a circle keeping it within the synchrotron. Synchrotrons offer an advantage in that due to the circular configuration, the particles can be accelerated multiple times as they make several passes around the ring rather than just having a single pass through a linear accelerator. The disadvantage of using synchrotrons is energy loss due to synchrotron radiation. When a charged particle is accelerated, it radiates photons thus losing energy. To maintain the particles moving within a ring, they must be turned around the ring by magnetic fields. This turning is a form of acceleration, and, as a result, the particles lose energy via photon radiation as they move around the ring. This radiation limits the the total energy to which particles can be accelerated. The rate of photon emission depends on the mass of the particle and the rate of acceleration, so a large scale synchrotron like the Tevatron uses protons and anti-protons and a large radius to minimize the effects of synchrotron radiation. The Tevatron accelerates and collides beams of protons and anti-protons around a large ring with two collision points. Each collision point houses an experiment with its own detector. These two experiments are named D $\varnothing$ and the Collider Detector at Fermilab (CDF). For this analysis, we use the events recorded from the CDF detector. This chapter will describe the Tevatron accelerator, the particle beams produced in the Tevatron, and the machinery of the CDF detector. 


\subsection{The Tevatron Accelerator}

The Tevatron accelerates beams of protons and anti-protons to an energy of $980 \mathrm{GeV}$ to provide $\sqrt{s}=1.96 \mathrm{TeV}$ collisions. The machine produces and stores anti-protons until there are enough to begin collisions. The anti-proton and proton beams are then injected into the main Tevatron ring for what is known as a "store" or a data run. As the beams circulate, the number of particles in them decreases due to collisions and beam losses. Collisions are generally continued until the number of particles remaining in the beams, measured by the instantaneous luminosity, is such that the rate of interesting physics is significantly reduced. While collisions are ongoing, the Tevatron again produces and stores anti-protons in anticipation of the next store.

The Tevatron accelerator chain [24] (see Fig. 2.2) begins with hydrogen gas being split into atoms and ionized to $H^{-}$. These hydrogen atoms are accelerated via a Cockcroft-Walton accelerator to $750 \mathrm{KeV}$. The ions are then passed on to a linear accelerator (called the "Linac") where they are accelerated to $400 \mathrm{MeV}$. All accelerators in the chain beginning with the Linac use Radio Frequency (RF) cavity acceleration. In $\mathrm{RF}$ acceleration, charged particles pass through cavities which are sized to support standing wave modes of RF electromagnetic waves. These standing wave fields boost the particles energy as they pass through the cavity. The $400 \mathrm{MeV}$ ions from the Linac are injected into the Booster. At the injection point, a stripper foil is used to remove the electrons leaving just the hydrogen nuclei which are bare protons. These protons are then accelerated to $8 \mathrm{GeV}$ in the Booster, a 75 meter radius synchrotron. The next step for these protons is the Main Injector. The Main Injector is another synchrotron which has a circumference 7 times that of the Booster and just over half the circumference of the Tevatron. The Main Injector can accelerate the protons up to $150 \mathrm{GeV}$. At this point, the protons can be used for one of two things. If the protons are to be used for $p \bar{p}$ collisions, they are accelerated to $150 \mathrm{GeV}$ and passed to the Tevatron. However, most of the time, the Main Injector is used to pass protons to a fixed target for anti-proton production [25]. In this case, the protons are accelerated to $120 \mathrm{GeV}$ before being passed to the anti-proton target station. 
At the target station, the protons collide with a fixed nickel alloy target to produce an array of particles including anti-protons. A magnetized Lithium lens is used to focus the outgoing particles into a beam and then a magnetic field is used like a spectrometer to separate particles of different momentum and electric charge. The end result is a beam of anti-protons with an energy of $8 \mathrm{GeV}$. These anti-protons are passed to the Debuncher, a triangular synchrotron with a mean radius of $90 \mathrm{~m}$. In the Debuncher, stochastic cooling is used to lower the transverse momentum spread of the anti-protons in the beam. Next, the anti-protons are stored in the Accumulator, a triangular synchrotron with a mean radius of $75 \mathrm{~m}$, which is housed in the same tunnel as the Debuncher. The purpose of the Accumulator is to simply store and further cool the anti-proton beam. The beam is not accelerated above $8 \mathrm{GeV}$ in either the Debuncher or Accumulator. When the Accumulator begins to fill with anti-protons, the beam is passed to the Recycler, another $8 \mathrm{GeV}$ synchrotron housed along the ceiling of the Main Injector tunnel. The Recycler employs stochastic cooling to further reduce the transverse energy spread of the anti-proton beam and electron cooling to reduce the spread in the longitudinal momentum of the beam producing more focused bunches of anti-protons for use in the Tevatron. The main function of the Recycler however is to store anti-protons and reduce the population of anti-protons in the Accumulator as the rate of anti-proton production decreases as the Accumulator saturates. The production of anti-protons is the main limiting step in the efficiency of the Tevatron as it can take 20 or more hours to produce enough anti-protons to begin a run of collisions in the Tevatron. Generally, anti-protons are produced and stored using the accelerator complex while a run of collisions are taking place in the Tevatron. However, long delays in data taking can occur if the Tevatron beams are lost before enough anti-protons are produced for the next run or if the stored anti-protons are lost before they can be used for collisions. When enough anti-protons have been produced, and we wish to begin a new run of collisions, the stored anti-protons are transferred from the Recycler to the Main Injector. This is where the advantage of using anti-protons becomes apparent. Because anti-protons have the same mass but opposite charge of protons, the same machines and magnetic fields used to accelerate and turn the proton beam can also be 
used to accelerate and turn the anti-proton beam in the opposite direction. Just as the $8 \mathrm{GeV}$ protons were accelerated, the Main Injector accelerates the $8 \mathrm{GeV}$ anti-proton beam to $150 \mathrm{GeV}$ in preparation of sending the beam to the Tevatron to begin collisions.

To begin a new data run, first the $150 \mathrm{GeV}$ anti-protons are injected into the Tevatron from the Main Injector. Once these are stable, protons are produced via the accelerator complex until a $150 \mathrm{GeV}$ beam is circling in the Main Injector. This proton beam is also injected into the Tevatron, but it travels in an opposite direction to the anti-proton beam. When the Tevatron is viewed from above, the protons travel clockwise around the detector and the anti-protons travel counterclockwise. Again, due to the difference in electric charge but an identical mass between protons and anti-protons, these two beams are circulated and accelerated using the same magnetic field and RF cavity setup in the Tevatron. Superconducting magnets apply a $4.2 \mathrm{~T}$ magnetic field to steer the beams which are accelerated to an energy of $980 \mathrm{GeV}$. The two beams thus collide with a $\sqrt{s}$ of $1.96 \mathrm{TeV}$. Additional quadrapole magnets are used to squeeze the transverse width of the beams before the two interaction points where the detectors are located. The proton and anti-proton beams are structured into 3 groups with 12 bunches of particles, giving 36 bunches in each beam. Within each group, the bunches are separated by $396 \mathrm{~ns}$ which gives a collision rate of $2.5 \mathrm{MHz}$. Between the groups, large empty gaps are left. When the beam is to be dropped at the end of a data run, these "abort gaps" are lined up with the detectors to ensure that neither detector is sprayed with a large amount of potentially damaging radiation.

The instantaneous luminosity of collisions, $\mathcal{L}_{\text {inst }}$, is calculated as:

$$
\mathcal{L}_{\text {inst }}=\frac{f N_{p} N_{\bar{p}} N_{b}}{4 \pi \sigma_{x} \sigma_{y}}
$$

where $\mathrm{f}$ is the collision rate, $N_{p}$ and $N_{\bar{p}}$ are the numbers of protons and anti-protons per bunch, $N_{b}$ is the number of bunches, and $4 \pi \sigma_{x} \sigma_{y}$ represents the effective collision area of the beams assuming each beam obeys a Gaussian distribution of particles in both the $\mathrm{x}$ and $\mathrm{y}$ direction with width $\sigma_{x}$ and $\sigma_{y}$ in the $\mathrm{x}$ and y directions, respectively. The $\mathrm{x}$ and $\mathrm{y}$ directions are defined in the transverse plane which is perpendicular to the beam direction $(\mathrm{z})$. Once data taking has commenced, the beams are usually kept 
circulating until beam losses and previous collisions cause the instantaneous luminosity to drop below a reasonable level for interesting physics to occur. We track the overall performance of the Tevatron and describe the amount of data taken with the integrated luminosity, $\mathcal{L}$, which is simply the integral of $\mathcal{L}_{\text {inst }}$ over a given time period. For any given process we wish to observe, the number of events in a dataset of luminosity $\mathcal{L}$ is simply $\mathcal{L} \cdot \sigma$, where $\sigma$ is the cross section of the process. As of September 15, 2011, the Tevatron has delivered $11.7 \mathrm{fb}^{-1}$ of data to the CDF detector (see Fig. 2.1).

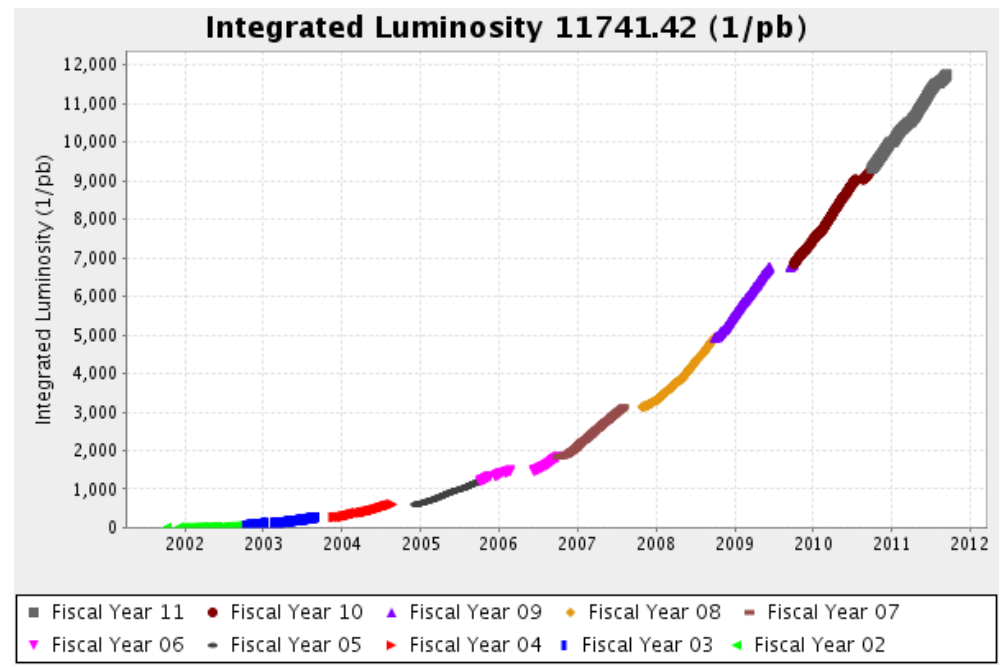

Figure 2.1: Integrated luminosity (in $\mathrm{pb}^{-1}$ ) delivered by the Tevatron as of Sept. 15, 2011 [26].

\subsection{The CDF Detector at Fermilab}

The CDFII Detector [28] is an upgrade of the original CDF detector [29] for RunII at Tevatron which began in 2001 and will continue until Sept 30th 2011. It is a multipurpose particle detector used to identify and measure the momentum or energy of particles produced from $p \bar{p}$ collisions. The detector is made up of tracking systems which identify the path of charged particles and calorimeters which measure the energy of particle showers. The innermost detector is a high precision silicon tracker which contributes to the tracking of charged particles and identifies secondary vertices from $b$ quarks which travel a short distance from the interaction point before hadronizing. 
FERMILAB'S ACCELERATOR CHAIN

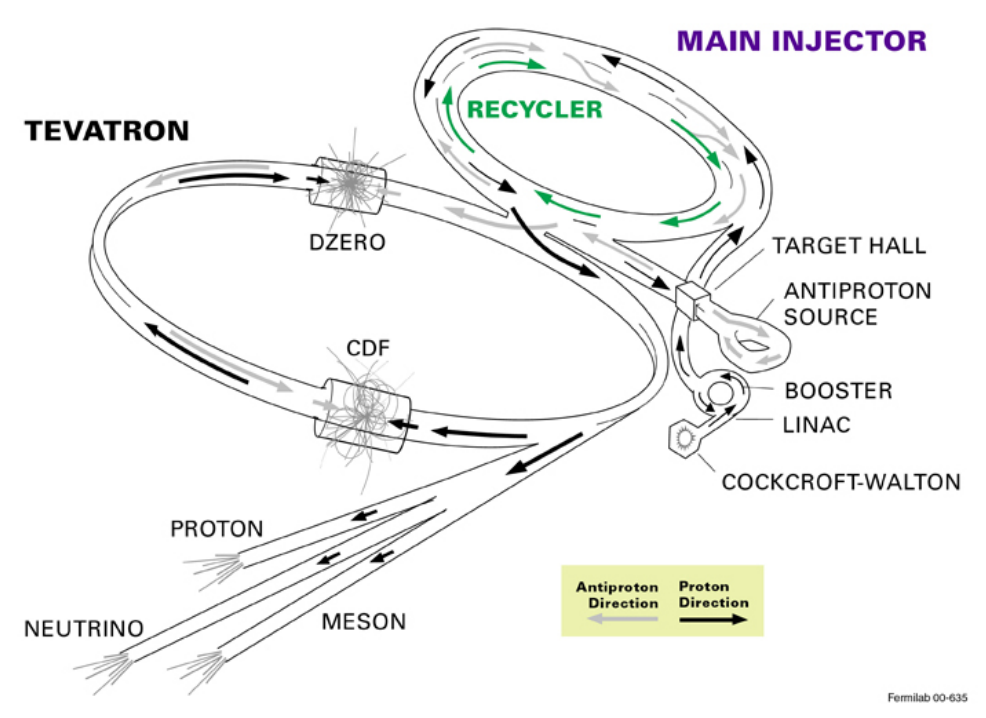

Figure 2.2: The Fermilab accelerator chain [27].

Outside of this is an open cell drift chamber tracker. A magnetic field is generated over these tracking regions from a solenoid outside of the tracker. This magnetic field causes charged particles to follow curved trajectories through the trackers. The curvature can then be measured and used to determine the momentum of the particle. Outside of the solenoid is a large $\mathrm{Pb} /$ scintillator calorimeter followed by a $\mathrm{Fe} /$ scintillator calorimeter for measuring energy showers of electromagnetic and hadronic particles, respectively. Finally, the last layer of the detector is composed of wire chambers and scintillator strips to provide tracking information for $\mu$ 's, the only charged particle which is not expected to be stopped by the calorimeters as it is a minimum ionizing particle. An elevation view of the CDF detector with these systems labeled is shown in Fig. 2.3. The detector is cylindrically shaped and obeys the same general symmetries as a standard cylinder except for a few instances where the symmetry must be broken either to provide cabling, coolant, or support to detector components or on account of the shape of the collision hall where the detector is located. The following subsections will describe the coordinate system of the CDF detector and its various detector subsystems. Figure 2.7 shows two cut away views of the CDF detector with these subsystems labeled. 


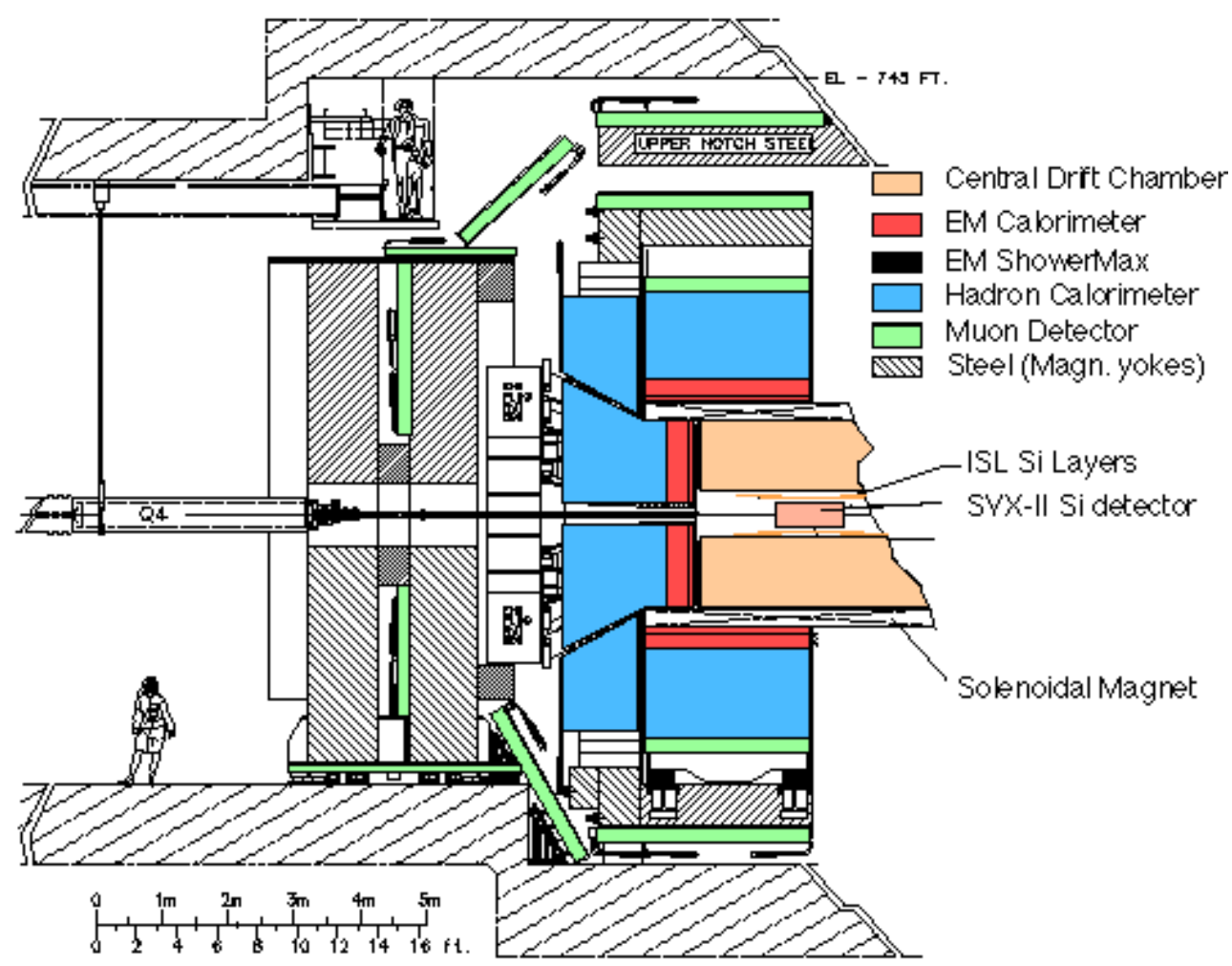

Figure 2.3: An elevation view of one half of the CDF detector. The silicon and central trackers, calorimeters, and muon detector systems described in this section are all labeled [30]. 


\subsubsection{Coordinate System}

The CDF detector is cylindrically shaped, and it is generally described with a cylindrical coordinate system. The z-axis runs along the beamline through the center of the detector. The $\mathrm{z}$ coordinate is defined to be 0 at the interaction point at the very center of the detector. It is positive towards the East, in the direction of proton travel, and negative to the West, in the direction of anti-proton travel. The r coordinate describes the radial distance from the beamline perpendicular to $\mathrm{z}$, and $\phi$ is the azimuthal angle around the beamline. The angle $\phi$ is defined so that North is 0 degrees, up is 90 degrees, etc. In spherical coordinates, we replace $\mathrm{z}$ with the polar angle $\theta$ where 0 degrees is along the positive $\mathrm{z}$ axis, 90 degrees points straight up, 180 degrees is along the negative $\mathrm{z}$ axis. The plane defined by $\mathrm{r}$ and $\phi$ is transverse to the beamline and therefore, is called the transverse plane. This plane can also be described by an x-y axis which we use when discussing a component of a particle's momentum in the transverse plane $\left(P_{T}\right)$. In $\mathrm{x}-\mathrm{y}$ system, $\mathrm{x}$ runs positive to the North and y runs positive upwards. For a particle with momentum $\mathrm{P}$, the individual components of the particle's momentum are defined using the spherical coordinates given above to be:

$$
\begin{aligned}
P_{T} & =P \cdot \sin \theta=\sqrt{P_{X}^{2}+P_{Y}^{2}}, \\
P_{X} & =P \cdot \sin \theta \cos \phi, \\
P_{Y} & =P \cdot \sin \theta \sin \phi, \\
P_{Z} & =P \cdot \cos \theta .
\end{aligned}
$$

Finally, when discussing the location of a particle in the CDF detector, we often use pseudorapidity $(\eta)$ rather than the spherical coordinate $\theta$. Pseudorapidity, $\eta$, is defined as $-\ln \tan \frac{\theta}{2}$. As a result, $\eta$ is zero at exactly 90 degrees to the beamline, and goes to $\pm \infty$ as the beamline is approached. We prefer to use $\eta$ because, for a particle with momentum which is much greater than its mass (as is the case for most final state particles observed in the $\mathrm{CDF}$ detector), $\eta$ is equivalent to rapidity, $\mathrm{Y}$, which is defined as:

$$
\mathrm{Y}=\frac{1}{2} \ln \left(\frac{E+P_{Z}}{E-P_{Z}}\right)
$$


where $E$ and $P_{Z}$ are the energy and z component of the particle's momentum, respectively. We make this choice because unlike theta, rapidity (and therefor $\eta$ for particles with negligible mass) is invariant under boosts along the beamline.

\subsubsection{Silicon Tracking}

The first part of charged particle tracking is handled by a silicon microstrip detector [31. This detector is used for precision tracking and the identification of secondary vertices which are used to identify heavy flavor quarks. The silicon detector is made of three components: Layer 00 (L00), the Silicon Vertex Detector (SVXII, to distinguish from the Run I detector SVX), and the Intermediate Silicon Layer (ISL).

The SVXII detector [32] is the main component of the silicon detector. It is composed of 5 layers (numbered 0 to 4 ) of double-sided silicon strips. The layers are spaced from an $\mathrm{r}$ of $2.1 \mathrm{~cm}$ to $17.3 \mathrm{~cm}$ and cover an $\eta$ range of \pm 2 (see Fig. 2.4). For full 3 dimensional tracking, the silicon strips on one side of each layer are aligned axially, and on the other side they are aligned with either a 90 degree (layers 0,1 , and 3 ) or 1.2 degree stereo angle. The SVXII detector is arranged in 3 barrels which altogether cover out to $45 \mathrm{~cm}$ in $\mathrm{z}$ in either direction from the interaction point.

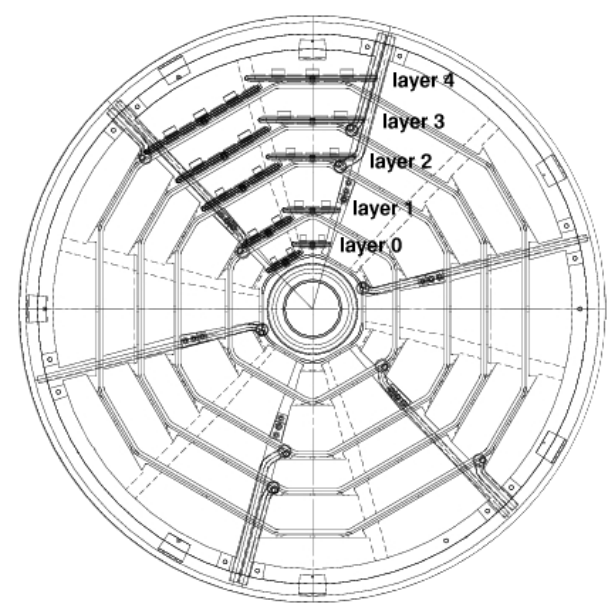

Figure 2.4: Bulkhead view of the SVX module showing the location of the silicon detector placement in layers 0 through 4 [33].

The L00 detector [34] is an additional silicon detector layer with additional radiation 
protection mounted directly onto the outside of the beampipe. It improves the tracking precision of the silicon detector as well as acts as insurance against the loss of silicon strips in layer 0 of the SVXII detector from radiation damage. It is mounted at $1.6 \mathrm{~cm}$ in $\mathrm{r}$ and covers an $\eta$ out to 4.0 in either direction. As the layer is only single-sided, it has no stereo mounted strips and only provides measurements in $\phi$.

Finally, the ISL detector [35] is mounted outside of the SVXII detector and is used to provide additional silicon tracking especially for particles traveling an at $|\eta|>1.0$ which will not traverse the entire $\mathrm{r}$ region of the COT detector to be described in Sec. 2.2 .3 It is composed of a single central layer at an $\mathrm{r}$ of $22 \mathrm{~cm}$, and two layers each in the forward and backward regions $1.0<|\eta|<2.0$ at r's of 20 and $28 \mathrm{~cm}$. Like the SVXII detector, these layers are double sided with an axial arrangement on the inner side and a small stereo angle arrangement on the outer side. Its placement between the SVX and COT detectors is shown in Fig. 2.5

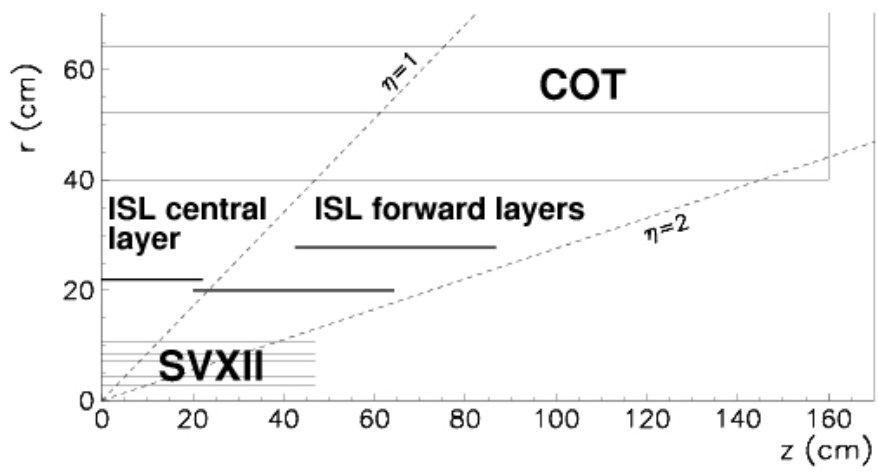

Figure 2.5: Side view of the inner tracking region of the CDF detector showing placement of the SVXII, ISL, and COT detectors [33].

The combined silicon detectors offer an $\mathrm{r}-\phi$ resolution of $11 \mu \mathrm{m}$, and an impact parameter resolution of $\sim 40 \mu \mathrm{m}$. The majority of the impact parameter resolution comes from the transverse size of the Tevatron beamline.

\subsubsection{Central Outer Tracker}

The second part of the CDF tracking system is the Central Outer Tracker (COT) [36]. The COT is a open cell drift chamber with full coverage of $|\eta|<1.0$ and partial coverage 
for $1.0<|\eta|<2.0$ (see Fig. 2.5). The COT extends from an $\mathrm{r}$ of $40 \mathrm{~cm}$ to $137 \mathrm{~cm}$. It is composed of 2520 cells arranged into 8 superlayers. Half of the superlayers (layers $2,4,6$, and 8) are arranged axially while the other 4 layers (layers 1, 3, 5, and 7) are arranged at a 2 degree stereo angle for resolution along the $\mathrm{z}$ axis. Each cell contains 12 sense wires and 13 potential wires. The COT is filled with a 50/50 mixture of argon and ethane gas chosen to minimize drift time. The design of the COT leads to an electron drift time of less than 200 ns. As bunch crossings in the beam happen approximately every $400 \mathrm{~ns}$, this drift time is sufficient to avoid any crossover in COT readings between events. For tracks with $|\eta|<1$, the tracking identification of the COT is nearly $100 \%$ for all tracks with a transverse momentum $\left(P_{T}\right)$ greater than $1 \mathrm{GeV}$ and $\sim 95 \%$ for tracks with lower $P_{T}$ [36].

Outside of the COT is a superconducting solenoid which provides the magnetic field for measuring the momentum of charged particles. The solenoid provides a $1.4 \mathrm{~T}$ magnetic field in the negative $\mathrm{z}$ direction. The magnetic field curves the trajectory of charged particles proportionally to their momentum. This allows us to calculate the direction and radius of curvature to ascertain the charge and the momentum of the particle, respectively. Specifically, the momentum of a particle in $\mathrm{GeV}$ is calculated as:

$$
P_{T}=0.3 q B R,
$$

where $\mathrm{q}$ is the particle's charge in units of the electron charge, $\mathrm{B}$ is the magnetic field in Tesla, and $\mathrm{R}$ is the particle's radius of curvature in meters. The COT alone can measure a particle's momentum up to $0.15 \% \cdot P_{T}$. Combined with the tracking information from the silicon tracker, this resolution is reduced to $0.07 \% \cdot P_{T}[36]$.

\subsubsection{Calorimeters}

Calorimeters are used to measure the energy and position of electromagnetic and hadronic particles in the detector. There should be as little material in front of the calorimeter as possible to avoid particles radiating energy before they reach it. However, since the calorimeters stop most particles, they are placed outside the tracking region. The central calorimeters are arranged cylindrically around the detector. 
Additional "plug" or forward calorimeters cover the bases of the cylindrical shape to maximize the coverage of the detector. The CDF calorimeters are made of alternating layers of absorbing material and scintillators. The absorbing material causes the particles to lose their energy either through collisions or radiation and the resulting showers are measured via the scintillating material. The energy in these showers can be used to measure the original particle's energy. Such calorimeters are called "sampling" calorimeters as opposed to "homogeneous" calorimeters in which the entire calorimeter is made of scintillating material which is also responsible for providing the parton showers. "Homogeneous" calorimeters give better energy resolution as the entire energy shower is contained within the scintillator material. "Sampling" calorimeters, on the other hand, are able to stop a high energy particle over a much shorter length due to the additional absorber material. The innermost calorimeter is made up of alternating lead/scintillator layers designed to stop electromagnetic particles (specifically electrons and photons) and is referred to as the electromagnetic calorimeter. The outermost calorimeter, called the hadronic calorimeter, uses iron/scintillator layers designed to stop hadrons.

When a high energy electron or $\gamma$ enters a material, it begins an electromagnetic shower. The $\gamma$ 's interact with an atomic nucleus or electron to produce an electron-positron (anti-electron) pair. Meanwhile, electrons (or positrons) emit $\gamma$ 's via Bremsstrahlung radiation. This radiation is the result of the charged electron being deaccelerated via its interaction with another charged object. These two processes chain together to form an electromagnetic shower from either a initial $\gamma$ or electron as they pass through the absorption material in the calorimeter. The shower continues until the remaining particles have low enough energy that they begin to lose energy via Compton scattering and are absorbed by the atoms of the material. It should be noted that, since $\mu$ 's have a significantly higher mass than electrons, they emit a minimal amount of Bremsstrahlung radiation, and as a result, they pass through the calorimeter with minimal energy deposit. The $\mu$ identification will be discussed in Sec. 2.2 .5

The electromagnetic calorimeter depth is measured by the parameter $X_{0}$ which is 
defined as the distance through a given material an electron passes before losing all but $\frac{1}{e}$ of its initial momentum. This also corresponds to $\frac{7}{9}$ of the mean free path for $e^{-} e^{+}$ pair production of a high energy $\gamma$. The choice of lead and iron for the electromagnetic and hadronic calorimeters gives $X_{0}$ of $5.5 \mathrm{~mm}$ and $1.7 \mathrm{~cm}$, respectively [37].

Hadrons traveling through material shower mainly via strong force interactions with the atomic nuclei in the material. In these showers, the interaction between the hadron and nucleus produces several less energetic hadrons which continue to interact with other nuclei. This shower continues until all particles are stopped or absorbed by the material. The hadronic shower depth is measured by the interaction length $\lambda$ which is the mean free path a hadron travels in a material before interacting with a nucleus. The length $\lambda$ in both lead and iron is $\sim 17 \mathrm{~cm}$ [37.

Lead and iron were chosen as the absorption material for the electromagnetic and hadronic calorimeters, respectively, due to their values for $X_{0}$ and $\lambda$. As $X_{0} \ll \lambda$, the electromagnetic calorimeter is placed in front of the hadronic calorimeter. The smaller value of $X_{0}$ for lead makes it the better candidate for the electromagnetic calorimeter. The electromagnetic calorimeters are built to be approximately $20 X_{0}$, but only $1 \lambda$, in depth. This ensures that the majority of electromagnetic showers occur within the electromagnetic calorimeter while the hadronic showers generally begin in the hadronic calorimeter.

The total calorimeter system at CDF is made up of 5 subsystems. The radiation depth and energy resolution for each of the 5 calorimeter subsystems is listed in Tab. 2.1. The electromagnetic calorimeter is composed of the central electromagnetic calorimeter (CEM) and the plug electromagnetic calorimeter (PEM). The CEM [38] is cylindrically shaped and covers a range of $|\eta|<1$.1. It has 31 layers of lead and scintillator, and it is organized into separate readout "towers" in $\eta-\phi$ space which each point towards the center of the detector. There are 24 tower wedges in $\phi$ and 10 towers in $\eta$ on each side of the detector. The forward region of the detector is covered by the PEM [39] which is circularly shaped and positioned along either side of the barrel end of the COT. The PEM covers $1.1<|\eta|<3.6$. It is composed of 23 layers of lead and scintillator, and arranged into 12 towers in $\eta$. The 4 innermost towers are composed of 
24 wedges in $\phi$, and the 8 outermost towers are composed 48 wedges in $\phi$. The CEM and PEM calorimeters also have additional "ShowerMax" (CES for central and PES for plug) detectors built into them about $6 X_{0}$ deep where the electromagnetic shower is expected to be at its maximum. These detectors measure the transverse profile of the EM shower which is useful for particle detection such as identifying two separate electromagnetic showers from a $\pi^{0} \rightarrow \gamma+\gamma$ decay. This is particularly important for measuring the energy of hadronically decaying $\tau$. The CES detector is made up of wire chambers and scintillator strips and the PES detector is made of a crossed array of scintillator strips.

The energy scale of the EM calorimeters is calibrated by matching the invariant mass of $e^{+} e^{-}$pairs to the known $Z$ mass $91.188 \pm 0.002 \mathrm{GeV}[3]$. First, the CEM scale is set by $e^{+} e^{-}$pairs where both electrons are in the CEM. Once the CEM is calibrated, the PEM is calibrated by studying $e^{+} e^{-}$pairs with one electron in the CEM, and the other in the PEM. This is done separately for East and West side PEM's. The time dependence of the calibration of the CEM is studied by following the ratio of the calorimeter measured energy and the tracking system (COT and silicon detectors) measured momentum $(E / p)$ for electrons with $E_{T}>8 \mathrm{GeV}$. This is useful for energy calibration because of the very small momentum resolution from the tracking detectors. For the PEM calorimeter, the time dependence of the energy scale is tracked via calibration runs using a laser system to test the response of the photomultiplier tubes (PMT's) and a radioactive $C o^{60}$ source to test the response of the PMT's and scintillator material. The calibration is adjusted as necessary to maintain a constant energy scale as the calorimeter components age [40].

The hadronic calorimeter is made up of three subsystems. The central hadronic calorimeter (CHA) [41] and the wall hadronic calorimeter (WHA) [41] cover the central region while the plug hadronic calorimeter (PHA) [39] covers the forward region of the detector. All three systems are composed of alternating layers of iron and scintillator. The CHA has 31 layers of iron and scintillator and covers $|\eta|<0.9$. The WHA is made up of 15 layers and slightly overlaps the CHA, covering $0.8<|\eta|<1.2$. Finally, the PHA has 23 layers and covers the forward region $1.2<|\eta|<3.6$. 


\begin{tabular}{|c|c|c|c|c|}
\hline \multirow{2}{*}{\multicolumn{2}{|c|}{\begin{tabular}{|c|} 
Electromagnetic \\
Coverage \\
Depth \\
Resolution $(\%)$
\end{tabular}}} & CEM & \multicolumn{2}{|c|}{ PEM } \\
\hline & & $\begin{array}{c}|\eta|<1.1 \\
19 X_{0}, 1 \lambda \\
\frac{13.5}{\sqrt{E_{T}}} \oplus 2\end{array}$ & $\begin{array}{r}1.1< \\
21 X \\
\frac{16}{\sqrt{E}}\end{array}$ & $\begin{array}{l}\eta \mid<3.6 \\
0,1 \lambda \\
=\oplus 1\end{array}$ \\
\hline Hadronic & CHA & $\mathrm{WH}$ & & PHA \\
\hline $\begin{array}{c}\text { Coverage } \\
\text { Depth } \\
\text { Resolution (\%) }\end{array}$ & $\begin{array}{c}|\eta|<1.1 \\
4.5 \lambda \\
\frac{50}{\sqrt{E_{T}}} \oplus 3\end{array}$ & $\begin{array}{r}0.8<\mid \eta \\
4.5 \\
\frac{75}{\sqrt{E_{T}}}\end{array}$ & $\begin{array}{l}<1.2 \\
\oplus 4\end{array}$ & $\begin{array}{c}1.2<|\eta|<3.6 \\
7 \lambda \\
\frac{80}{\sqrt{E_{T}}} \oplus 5\end{array}$ \\
\hline
\end{tabular}

Table 2.1: Energy resolution, radiation depth, and $\eta$ coverage for each of the 5 calorimeter subsystems [38] [39] [41],42].

The CHA and WHA are each arranged into 24 wedges in $\phi$, and 8 and 6 towers in $\eta$, respectively. Finally, the PHA has the same tower arrangement as the PEM except the outermost tower is not needed as it overlaps with the region covered by the WHA. The placement of the WHA and PHA calorimeters, as well as the PEM calorimeter, is shown in Fig. 2.6. The CHA, WHA, and PHA energy scale is initially set via data from a $50 \mathrm{GeV}$ charged pion test beam. The time dependence of the energy scale of the CHA and WHA is tracked with calibration runs using a laser system or a $C s^{137}$ radioactive source. Additionally, muons from $J / \psi \rightarrow \mu^{-} \mu^{+}$decay and minimum bias data (min-bias) are also used to track the energy scale. Min-bias data is simply data selected by a trigger looking for simultaneous activity in the CLC luminosity detectors (to be described in Sec. 2.2.6). This ensures that the recorded event contains at least $1 p \bar{p}$ interaction, and it also avoids biasing the selection toward any triggerable process hence the name minimum bias. This dataset is excellent for studying the effects from multiple $p \bar{p}$ interactions within the same bunch crossing and the spectator partons in a $p \bar{p}$ interaction (known as underlying event). The PHA calorimeter energy scale time dependence is tracked in the same manner as the PEM energy scale defined above [40].

\subsubsection{Muon Detection Systems}

Due to their high mass of $105.658367 \pm 0.000004 \mathrm{MeV}$ [3], $\mu$ 's emit a minimum amount of Bremsstrahlung radiation. For this reason, the $\mu$ is said to be a minimum ionizing particle, and it passes through the calorimeters with minimal energy deposit. This 


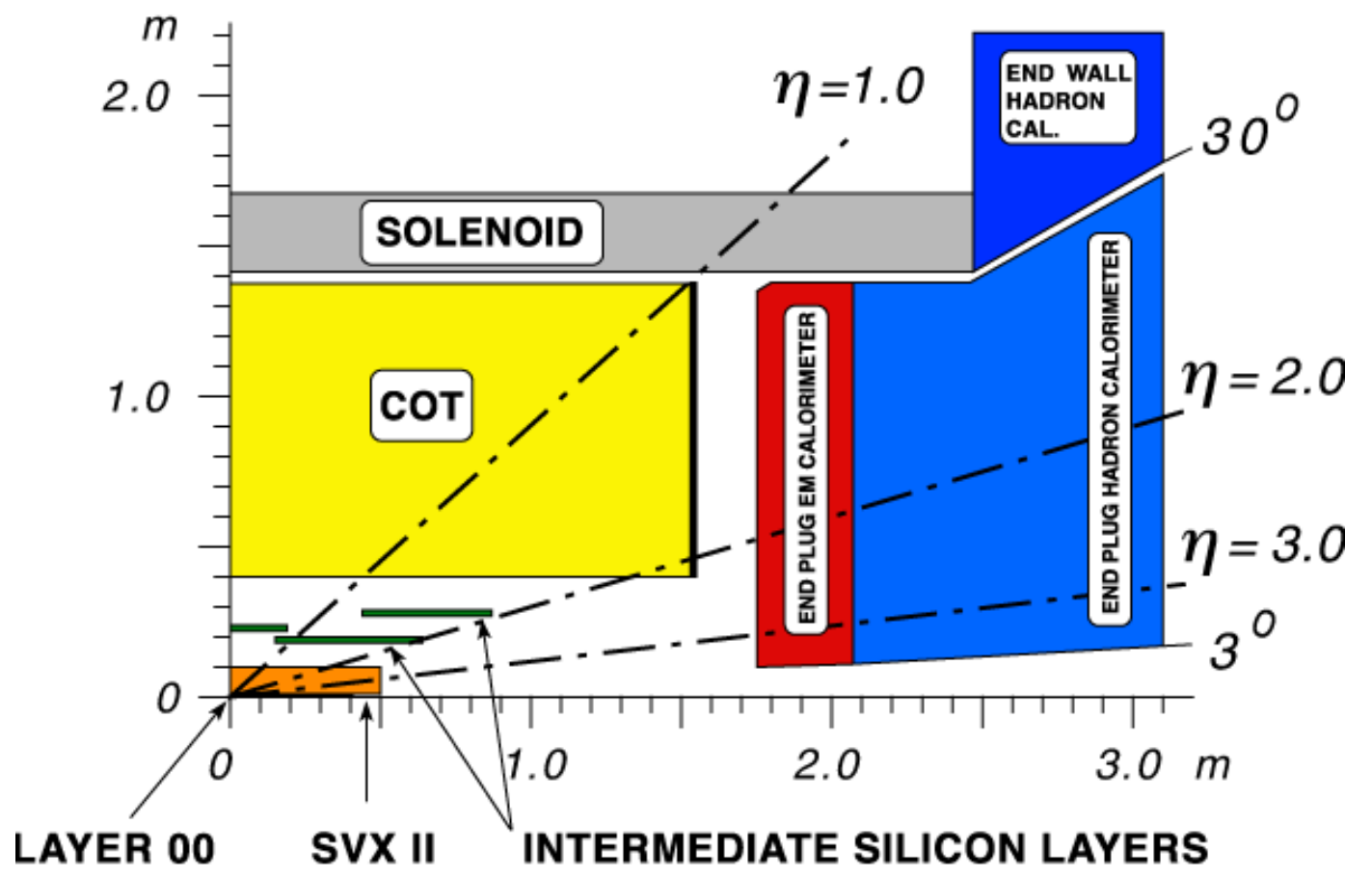

Figure 2.6: Side view showing the placement of the inner tracking detectors and the plug and endwall calorimeters. The CEM and CHA calorimeters are not shown but are located directly outside of the solenoid [43]. 
means that the $\mu$ and $\nu$ are the only two SM particles expected to continue past the CDF detector. The $\nu$ 's of course have very low interaction cross sections which makes them impossible to detect at $\mathrm{CDF}$ with any reasonable efficiency. However, we improve the $\mu$ detection by placing wire drift chambers outside of the CDF detector to identify the tracks of $\mu$ 's which have passed through the CDF calorimeters. The $\mu$ detection is accomplished via wire drift chambers with a layer of scintillator tiles for fast timing and trigger information since the drift time for $\mu$ 's in these detectors can be longer than the time between bunch crossings [44]. The tracks that a $\mu$ leaves in the muon chambers are referred to as a "stub". These stubs are matched to extrapolated tracks from the COT to verify $\mu$ detection, and CDF $\mu$ 's are described by the subsystem of the $\mu$ detectors in which their stub is found. The central muon detector (CMU) [45] is located directly outside of the hadronic calorimeter covering an eta range $|\eta|<0.6$. An upgrade to the central muon detector (CMP) [46] was put in place for Run II. It uses the steel return yoke of the solenoid as extra shielding to avoid misidentification of muons from charged particles which manage to pass through the calorimeter. As a result, it has a somewhat unique geometry at CDF. While most detectors are barrel shaped and obey general cylindrical symmetry, the CMP detector is box shaped around the outside of the return yoke. Like the CMU, it also covers a range of $|\eta|<0.6$. $\mu$ 's which leave stubs in both the CMU and CMP detectors are called CMUP $\mu$ 's. The central muon extension (CMX) [46] is a shaped like a conical section and extends the muon detector coverage from $0.6<|\eta|<1.0$. Finally, the barrel muon chambers (BMU) [46] are located cylindrically around the forward regions of the detector to provide $\eta$ coverage of $1.0<|\eta|<1.5$.

\subsubsection{Luminosity Counters}

To measure the luminosity of the collisions, the CDF detector uses a subsystem of Cherenkov Luminosity Counters (CLC) [47. These counters are located near the beamline at $3.7<|\eta|<4.7$ on either side of the detector. Each of the CLC modules is made up of 48 conical gas Cherenkov counters. These counters are arranged in three concentric circles with 16 counters each and are pointed back toward the interaction point. 
This reduces the background count as particles from $p \bar{p}$ interactions will traverse the full detector and lead to larger signals. Particles from beam halo or secondary production will cross the counters at an angle which leads to shorter paths in the counter and smaller signals. The detectors measure the number of inelastic $p \bar{p}$ collisions per bunch crossing, $\mu_{p \bar{p}}$. This quantity can be combined with the frequency of the bunch crossing, $f_{0}$, and the theoretical cross section for inelastic $p \bar{p}$ scattering, $\sigma_{p \bar{p}}$, to calculate the luminosity, $\mathcal{L}$ :

$$
\mathcal{L}=\frac{f_{0} \mu_{p \bar{p}}}{\sigma_{p \bar{p}}} .
$$

Both the measured number of collisions per bunch crossing and the theoretical cross section have uncertainties of about $4 \%$ [48. As a result, the calculated luminosity has an irreducible uncertainty of $6 \%$.
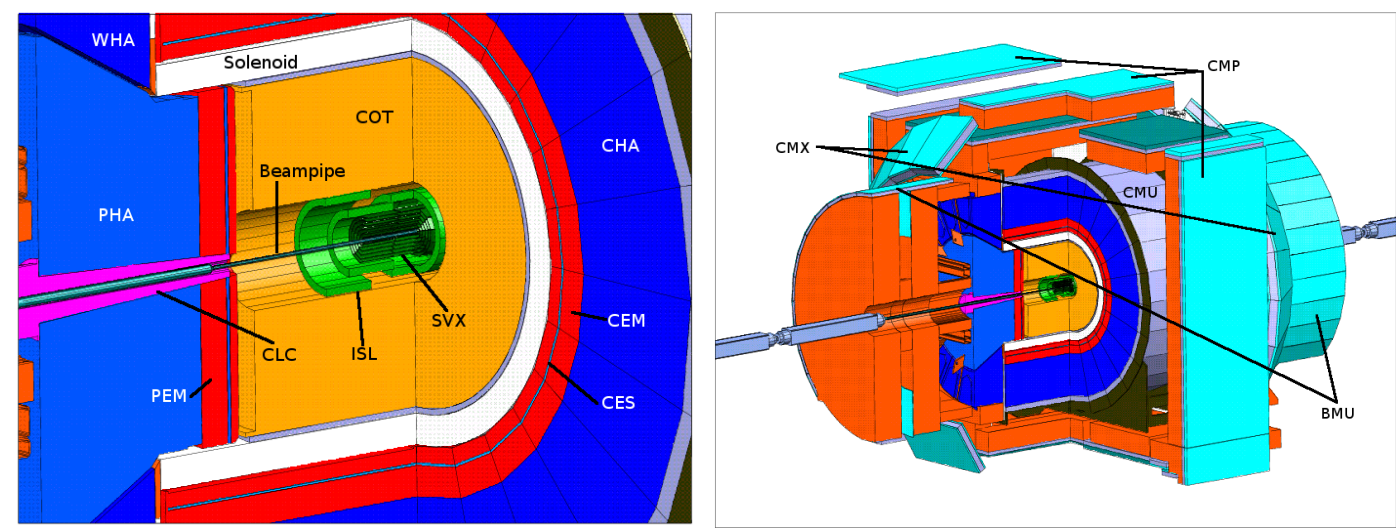

Figure 2.7: Diagrams of the CDF detector with relevant detector components labeled. The diagram on the left is zoomed in to highlight the tracking region. The diagram on the right is a full view and has the $\mu$ systems labeled [49].

\subsubsection{Trigger System}

One of the main challenges of operating the CDF detector is deciding which events to save for analysis. The Tevatron provides collisions at a rate of $1.7 \mathrm{MHz}$. However, the full readout of the $\mathrm{CDF}$ detector can only be stored to tape at a maximum rate of 75 $\mathrm{Hz}$. As a result, we must chose which events to keep while throwing out the majority 
of observed events. Most of the collisions are soft $p \bar{p}$ inelastic scattering which give little to no interesting physics at CDF. However, as there is a broad physics program at $\mathrm{CDF}$, we must design a triggering system to keep as many events as possible from a wide range of physics (top, electroweak, Higgs searches, exotic particles, etc.). The trigger must keep as many potentially interesting events as possible while also filtering events at a rate sufficient enough to avoid losing events due to backup of events either in the trigger path or being written to tape. To accomplish this, CDF uses a three tiered trigger system with each tier progressively filtering the events [50]. Each tier of the trigger sees fewer events due to the upstream tiers already filtering out events, therefore, the downstream tiers progressively use more information and take more time to make their decision.

The first tier, known as level 1 , is a pure hardware trigger. It makes a decision approximately $5 \mu \mathrm{s}$ after each collision. Its decision is based on readout from several detector components with little to no processing. It uses quick tracking reconstruction from the COT, single calorimeter tower information, event missing $E_{T}\left(E_{\mathrm{T}}\right.$, defined in Sec. 3.2.3), and muon stubs matched to COT tracks to look for electrons, $\mu$ 's, $\tau$ 's, $\nu$ 's, and jets which are indicative of interesting physics. The maximum acceptance rate is limited to $\sim 35 \mathrm{kHz}$ by the input rate of the level 2 trigger.

The level 2 trigger uses a combination of hardware and software to partially reconstruct events. It uses information from the silicon detector for improved tracking and identification of secondary vertices. It also reconstructs calorimeter clusters from multiple towers and incorporates the CES and PES detector information in electromagnetic showers. The level 2 trigger has a 4 event buffer to store additional incoming events from the level 1 trigger while processing a current event. If a fifth interesting event passes level 1 before a level 2 buffer becomes available, the event is lost. This deadtime is generally less than $5 \%$. The average processing time for the level 2 trigger is approximately $30 \mu \mathrm{s}$, and its maximum accept rate is $600 \mathrm{~Hz}$.

Finally, the level 3 trigger decides if an event should be written to tape. The trigger uses a large computer farm to fully reconstruct each event. It has a maximum acceptance rate of $75 \mathrm{~Hz}$. Events passing the level 3 trigger are written directly to 
tape for later analysis. The specific trigger requirements used to select events for this analysis will be described in Sec. 3.1 . 


\section{Chapter 3}

\section{Particle and Event Selection}

This chapter describes the experimental data used in this analysis and the selection requirements used to select the $\tau+$ jets candidate events with the CDFII detector. It includes a breakdown of the various particles of interest in the analysis along with descriptions of their detector signature and the selection requirements used to identify them. This analysis deals specifically with $\tau$ 's, jets from quarks, and $\nu$ 's. Additionally, we veto events with leptons other than the $\tau$. Finally, we will give the full requirements for hadronic $\tau+$ jets event selection. Although this chapter deals with experimental data, all simulated data (which will be described later in Sec. 4) must pass the same requirements. For this analysis, we use data corresponding to a total integrated luminosity of $2.2 \mathrm{fb}^{-1}$ collected with the CDFII detector between February 2002 and August 2007.

\subsection{Event Trigger}

The data is collected with the TOP_MULTI_JET trigger. This trigger selects events with 4 or more jets and large $\sum E_{T}$, as calculated by summing over the calorimeter clusters. For the sake of the trigger, the hadronically decaying $\tau$ is considered to be a jet, so the events we select appear as 5 jet events which helps to avoid the 4 jet turn-on in the trigger selection. The turn-on describes the shape of the average trigger acceptance as a function of some quantity. Ideally, the trigger will select all events with 4 or more jets, but due to reconstruction effects and the $E_{T}$ of each jet, it is possible that some jets do not pass the requirements needed to be counted by the trigger. The 5 jet events ( 4 jets plus a hadronic $\tau$ ) which are of interest in this analysis can have 1 jet fail to meet the trigger requirement and still pass the trigger selection. These events 
thus measure a higher trigger efficiency than 4 jet events in which the acceptance effect is greater. Additionally, since the trigger has no $\tau$ lepton selection requirements, there is minimal trigger bias in the $\tau$ selection. Unfortunately, due to the $\sum E_{T}$ requirement, this trigger is sensitive to the kinematics of the particular events we select. This means we cannot simply measure the trigger turn-on in data and weight the Monte Carlo (MC) simulated data appropriately. Instead, we must use MC simulation to measure the trigger efficiency for each process.

The TOP_MULTI_JET trigger has changed over time and has three different versions which were used to collect the data. In the first version, the level 1 trigger requires a single calorimeter tower with $\mathrm{E}_{T} \geq 10 \mathrm{GeV}$ indicative of a high $P_{T}$ jet, and the level 2 trigger requires 4 or more calorimeter clusters each with $E_{T} \geq 15 \mathrm{GeV}$ with the total $\sum E_{T}$ of all clusters to be greater than $125 \mathrm{GeV}$. The second version is identical except the $\sum E_{T}$ requirement at level 2 is moved up to $175 \mathrm{GeV}$. Finally, the third version is identical to the second version except the level 1 trigger now requires a single calorimeter tower jet with $E_{T} \geq 20 \mathrm{GeV}$. For simplicity sake, we remake the first version of the trigger requiring $175 \mathrm{GeV} \sum E_{T}$ thus making it identical to the second version. Since $t \bar{t}$ events generally decay to jets with large $\sum E_{T}$, this choice does not significantly reduce the number of signal events passing the trigger. In all three versions, level 3 of the trigger requires at least 4 reconstructed jets each with an uncorrected $E_{T}$ of $10 \mathrm{GeV}$ or greater.

For all MC, we model the full path of the trigger and assign each event a trigger weight to simulate the trigger efficiency. The original $10 \mathrm{GeV}$ level 1 requirement is found to be greater than $99 \%$ efficient when the level 2 trigger fires [51], so we ignore this requirement when modeling the trigger until the switch to a $20 \mathrm{GeV}$ requirement. For events with the level 1 requirement of $20 \mathrm{GeV}$, we use a function given in Eqn. 3.1 fit to the level 1 turn-on curve measured versus the $\sum E_{T}$ of all jets to weight the MC. The $\sum E_{T}$ of the jets is corrected as described in Sec. 3.2.2. This fit is described in detail elsewhere 52 and gives a level 1 scale factor $\left(S F_{l 1}\right)$ for all events selected with 
the third version of the trigger:

$$
S F_{l 1}=\frac{1}{1+\exp \frac{\left(153-\sum E_{T}\right)}{24.01}}
$$

Events selected from the first two versions of this trigger are assigned a scale factor of 1 , or unscaled.

Next, we check the level 2 requirement. First we apply run dependent level 2 calorimeter cluster energy corrections described in detail elsewhere [51] to all level 2 clusters in the MC. These corrections are necessary because it is found that, while the level 2 cluster $E_{T}$ scales linearly with the jet $E_{T}, \mathrm{MC}$ jets are found to give higher energy level 2 clusters than similar $E_{T}$ jets in data. As a result, if the MC cluster energies are not rescaled, MC events will be more likely to pass the trigger requirement than data events. We then check that each event has at least 4 level 2 clusters of $E_{T} \geq 15 \mathrm{GeV}$ and a total level $2 \sum E_{T}$ greater than the cutoff threshold. In CDF internal note 9954 [51], a level 2 data/MC scale factor $\left(S F_{l 2}\right)$ of 0.963 is measured for all events passing the level 2 requirements by measuring the ratio of the trigger turn on in data and MC as a function of the $\sum E_{T}$ of all jets. Since this ratio is found to have no dependence on $\sum E_{T}$ of the jets, we simply take the fitted value of $0.963 \pm 0.008$ to be $S F_{l 2}$.

Finally, we check the level 3 trigger requirement. The efficiency is measured as the percentage of events which have passed the level 1 and 2 trigger requirements which also pass the level 3 requirement. Like the level 1 requirement in the first two versions of the trigger, this level 3 trigger is found to be $\geq 99 \%$ efficient [51].

After simulating the trigger requirements, we assign the following trigger weight to each MC event:

$$
\text { Weight }=S F_{l 1} \times(\text { bool }) \text { Pass }_{l 2} \times S F_{l 2} \times(\text { bool }) \text { Pass }_{l 3}
$$

where the boolean variables $\mathrm{Pass}_{l 2}$ and $\mathrm{Pass}_{l 3}$ are 1 if the event passes the level 2 and level 3 triggers requirements, respectively, and 0 if it does not. 


\subsection{Particle Detection and Reconstruction}

For this analysis, we select events with exactly 1 hadronically decaying $\tau, 4$ jets with $E_{T} \geq 20 \mathrm{GeV}$, at least one of which must have a b-tag, and large missing $E_{T}\left(E_{\mathrm{T}}\right)$ indicative of a $\nu$. Section 3.2.1 describes the $\tau$ selection. Jet selection and a description of b-tagging are discussed in Sec. 3.2.2. Section 3.2.3 will define $E_{\mathrm{T}}$ and describe its relationship to $\nu$ 's. Finally, the full event selection requirements will be summarized in Sec. 3.3 .

\subsubsection{Taus}

The $\tau$ leptons decay either leptonically into an electron or muon and two $\nu$ 's or semihadronically into a jet of hadrons and a $\tau$ flavor $\nu$. The branching fractions for each of the various modes are listed in Tab. 1.1. The hadrons tend to be charged pions and neutral $\pi^{0}$ 's, however, kaons and other hadronic particles have a small probability of occurring in $\tau$ decays. Leptonically decaying $\tau$ 's are difficult to separate from standard electrons or muons, and they may already be included in other lepton + jet analysis (such as the one described in [53]). Therefore, we chose not to work with them. Instead, we focus on the hadronically decaying $\tau$ 's.

Due to their low mass compared to their energies, $\tau$ 's from $W$ and $Z$ boson decays appear in the CDF detector as narrow jets with an odd number of charged tracks and low $\pi^{0}$ multiplicity. Charged hadrons from the $\tau$ decay appear as charged tracks and energy showers in the hadronic calorimeter. Neutral $\pi^{0}$ 's decay into pairs of photons which give energy showers in the electromagnetic calorimeter. Charged tracks are identified and measured in the central tracker, and $\pi^{0}$ 's are reconstructed from the electromagnetic calorimeter clusters with positioning information coming from hits in the CES detector.

A $\tau$ candidate is seeded by a single calorimeter tower with $E_{T} \geq 6 \mathrm{GeV}$. Any adjacent towers with $E_{T} \geq 1 \mathrm{GeV}$ are added to form a calorimeter cluster. As the $\tau$ decay occurs in a relatively narrow cone, we limit the number of towers in the cluster to 6 . Next, we must find a seed track for the $\tau$. This track must point toward the calorimeter cluster and have a $P_{T}$ of at least $10 \mathrm{GeV}$. The seed track is required to have 
at least 5 hits in each of 3 or more axial and 2 or more stereo segments of the COT, an impact parameter in relation to the beamline $\left(d_{0}\right)$ of less than $1 \mathrm{~cm}$, and a $\mathrm{z}$ vertex position within $60 \mathrm{~cm}$ of the center of the detector. If there are multiple candidate seed tracks, we select the one with the highest $P_{T}$. This seed track is then used to draw two cones which define an inner signal cone and an outer isolation annulus as shown in Fig. 3.1. To improve jet rejection, the first cone size is variable depending on the energy of the calorimeter cluster. This cone, called the signal cone, extends to the minimum of 10 degrees and $(5 \mathrm{GeV}) / E_{c a l}$ radians where $E_{c a l}$ is the energy of the calorimeter cluster of the $\tau$ candidate. The second cone is used to define an isolation annulus around the $\tau$ and extends out to 30 degrees. All tracks with $P_{T} \geq 1 \mathrm{GeV}$ within these cones are considered. If the track is within the signal cone, it is considered part of the $\tau$. Tracks in the isolation annulus are evidence of another object decaying near a real $\tau$ or decaying with a similar signature to a real $\tau$. In either case, the $\tau$ object cannot be considered to be a clean $\tau$ signature, so we veto these objects. Neutral $\pi^{0}$ 's are reconstructed from the calorimeter cluster using positioning information from the CES detector. To ensure $\pi^{0}$ 's are well reconstructed, the z position of the $\tau$ object in the CES detector $\left(Z_{C E S}\right)$ must be within 9 and $230 \mathrm{~cm}$ of the center of the detector. If the $\pi^{0}$ falls within the $\tau$ signal cone, it is considered to be one of the $\tau$ decay products. However, if it falls within the isolation annulus, we do not include it in the $\tau$, and the $\tau$ candidate will likely fail the calorimeter isolation requirement.

We reconstruct the 4-momentum of the $\tau$ from all charged tracks and reconstructed $\pi^{0}$ 's in the signal cone. This quantity is known at the "visible" 4-momentum of the $\tau$. We select $\tau$ 's with visible $E_{T} \geq 25 \mathrm{GeV}$ and calorimeter cluster $E_{T} \geq 20 \mathrm{GeV}$. Both the calorimeter cluster and track $\eta$ 's must be in the central region of the detector (defined as $|\eta|<1)$. Since $\tau$ 's generally have a low track multiplicity and must decay to an odd number of charged hadrons to conserve charge, we require 1 or 3 charged tracks in the signal cone. We do not consider $\tau$ candidates with 5 or more tracks because, as shown in Tab. 1.1, the branching ratio for $\tau \rightarrow \mathrm{N}$ charged particles quickly becomes negligible above $\mathrm{N}=3$. To ensure we are selecting well isolated $\tau$ 's, we require that the calorimeter energy within the isolation annulus be less than $10 \%$ of the energy 
of the $\tau$. We veto electrons faking $\tau$ 's by requiring $\xi^{\prime} \geq 1.0$ where $\xi^{\prime}$ is defined as $\frac{E_{t o t}}{P^{t r k}} \times(0.95-$ EMfrac $)$, where $E_{t o t}$ is the total energy in the calorimeter cluster, $P^{t r k}$ is the scalar sum of the momentum of all tracks in the signal cone of the $\tau$, and EMfrac is the fraction of calorimeter energy measured in the electromagnetic calorimeter. While the exact formula may seem complicated, the idea behind this requirement is simple. If the hadronic calorimeter energy is much smaller than the total $\sum P_{T}$ of the charged tracks, then the tracks are most likely coming from an electron or muon rather than a charged hadron. Finally, the $\tau$ mass is $1.8 \mathrm{GeV}$, and we cannot fully reconstruct the $\tau$ due to the presence of a $\nu$. Therefore, we expect the majority of selected $\tau$ 's to have a mass below $1.8 \mathrm{GeV}$. Since the fraction of jets faking $\tau$ 's greatly increases above this threshold, we can further reject jets faking $\tau$ 's by requiring that the visible mass of the $\tau$ be less than $1.8 \mathrm{GeV}$. These $\tau$ selection requirements are summarized in Tab. 3.1 .

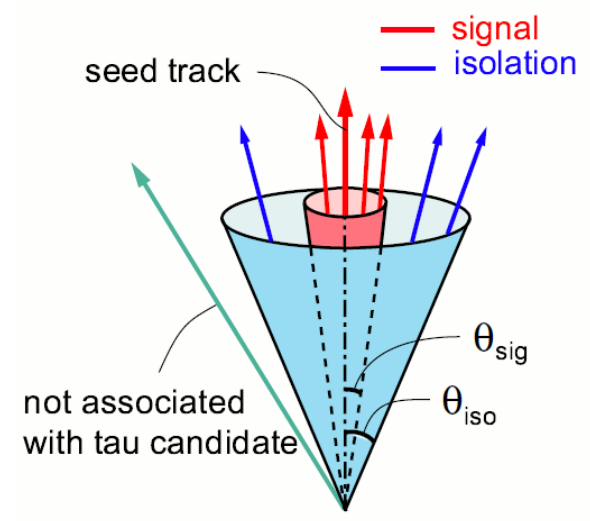

Figure 3.1: Diagram of $\tau$ reconstruction illustrating the signal cone and isolation annulus. $\theta_{\text {sig }}$ is defined to be the minimum of 10 degrees and $(5 \mathrm{GeV}) / E_{c a l}$ radians. $\theta_{\text {iso }}$ is always 30 degrees. This particular object has 4 signal tracks (red), and 3 tracks (blue) in the isolation annulus. It should be noted that this is not a good $\tau$ candidate, but rather just an illustration of the signal and isolation cone method used to identify $\tau$ 's .

\subsubsection{Jets}

In the SM, the strong force governs interactions between color charged quarks and gluons. Unlike the electromagnetic or weak forces, the strong force does not attenuate as the distance between quarks grows. Instead, as two bound quarks travel away from each 


\begin{tabular}{|c|c|}
\hline Kinematic Requirements & \\
\hline Visible $E_{T}$ & $>25 \mathrm{GeV}$ \\
\hline Cluster $E_{T}$ & $>20 \mathrm{GeV}$ \\
\hline Cluster $\left|\eta_{\text {det }}\right|$ & $<1$ \\
\hline Tracks $|\eta|$ & $<1$ \\
\hline$\left|Z_{C E S}\right|$ & $>9$ and $<230 \mathrm{~cm}$ \\
\hline Tracks in Signal Cone & 1 or 3 \\
\hline Visible Mass & $<1.8 \mathrm{GeV}$ \\
\hline Seed Track Requirements: & \\
\hline COT Segments & $\geq 3$ Axial $/ 2$ Stereo \\
\hline$P_{T}$ & $>10 \mathrm{GeV}$ \\
\hline$d_{0}$ & $<1 \mathrm{~cm}$ \\
\hline$\left|Z_{0}\right|$ & $<60 \mathrm{~cm}$ \\
\hline Isolation Requirements: & \\
\hline Tracks in Isolation Cone & 0 \\
\hline Calorimeter $E_{T}$ Isolation $/ \tau E_{T}$ & $<0.1$ \\
\hline Electron Removal & \\
\hline$\xi^{\prime}$ & $>0.1$ \\
\hline
\end{tabular}

Table 3.1: Tau identification requirements

other, the energy stored in the gluon field between the two quarks grows. The common metaphor to visualize this is two quarks connected by a spring. As the quarks separate and the energy in the field grows, eventually, it becomes energetically favorable for the energy stored in the field to produce a new $q \bar{q}$ pair which interact with the original two quarks rather than continuing to extend the distance of the field between the original two quarks. This process is called hadronization, and it leads to the principle of confinement which states that a color charged particle will not exist in a bare state but will rather always be bound in a color neutral state with other color charged particles [7.

As a result of this, bare quarks produced in the $t \bar{t}$ decay are not observed in the CDF detector. Instead, the quarks hadronize into a shower or jet of color neutral hadrons. The charged hadrons in the jet are observed in the silicon vertex and central trackers; however, since jets can contain several neutral hadrons as well, they are identified as energy clusters in the calorimeter within a $0.4 \eta-\phi$ cone (defined as $\sqrt{\eta^{2}+\phi^{2}}$ ). Jets are selected with a simple fiducial requirement on the jet's $\eta$ and a requirement on 
the jet's $E_{T}$. This causes jets to be a "catch-all" for any observed object which does not fit the requirements for electrons or $\tau$ 's. As a result, we ignore any jet which is reconstructed within a 0.4 cone in $\eta$ and $\phi$ of an identified electron or $\tau$.

\section{Jet Energy Corrections}

Jets are merely the reconstruction of the final state result after the hadronization of a quark, therefore the jet energy measured by the CDF detector is not a very good estimation of the energy of the original quark. To improve this, we apply several energy corrections to the reconstructed jet to account for the calorimeter response to different particles and different energies, gaps in the detector, underlying spectator particles, and radiation leaking outside of the reconstruction cone.

Specifically, we apply three main energy corrections to jets which are designed to map the raw reconstructed jet energy to the sum of the energy of all the hadrons produced in the hadronization of the initial quark which fall within the jet cone. These corrections are called the $\eta$-dependent (or relative), multiple interaction, and absolute corrections [40].

The $\eta$-dependent correction scales the energy of jets that fall outside of the $0.2 \leq$ $|\eta| \leq 0.6$ region to energy of jets within the region. Its main function is to correct for jet energy lost to the crack in the center of the calorimeter and the cracks between the central and plug calorimeters near an $|\eta|$ of 1.1 . The $0.2 \leq|\eta| \leq 0.6$ region is chosen as it lies within the well understood central calorimeters and is far from any uninstrumented areas of the CDF detector. This correction is derived from dijet events where one of the jets falls within the central $0.2 \leq|\eta| \leq 0.6$ region by assuming that the transverse energy of the two jets in a simple $2 \rightarrow 2$ interaction should be equal. This assumption is valid unless hard QCD radiation is present. Events must pass selection requirements based on the jet $P_{T}$ 's and the $E_{\mathrm{T}}$ significance (defined as $E_{\mathrm{T}}$ divided by the $\sum E_{T}$ of all jets) to veto events with QCD radiation. Ideally, the calorimeter response as a function

of $\eta$ should be flat after the correction, but due to the inability to remove all events with QCD radiation and imperfections in jet energy reconstruction, some fluctuations remain in the calorimeter response. The spread of these fluctuations combined with 
the change in response under fluctuations of the event selection requirements are used to define the systematic uncertainty on this correction [40].

The multiple interaction correction is used to remove energy from particles which are produced from additional $p \bar{p}$ interactions within the same bunch crossing as the desired event and which fall within the jet cone. This correction is applied as an average contribution derived from min-bias data (defined in Sec. 2.2.4 as data triggered by simultaneously activity in the East and West CLC detectors) and is parameterized in terms of the number of primary vertices observed in the event. The uncertainty on this measurement comes from reconstructing either too many or too few vertices. To estimate this effect, the correction is derived in min-bias data, JET100 triggered data (events triggered by a single jet with $E_{T} \geq 100 \mathrm{GeV}$ ), and $W \rightarrow e \nu_{e}$ events. Although no significant difference is observed between these samples, the uncertainty is derived from the limited statistics of the data samples [40].

Finally, the absolute correction is used to correct for any non-linear calorimeter response in jet energy and energy loss due to the jet cone overlapping with noninstrumented sections of the CDF detector. After this correction is applied, the jet energy scale is independent of the CDF detector. For this correction, a mapping function is derived between the reconstructed jet energy and the $\sum P_{T}$ of all particles within the jet cone (particle jet) by studying jets in MC with full simulation of the CDF calorimeters. The MC is validated to the data by studying the $P_{T}$ balance in $\gamma$-jet events. Since the $\gamma$ 's energy is reconstructed very well in the CDF detector, $\gamma$ 's provide an effective tool for studying jet balancing in data and $\mathrm{MC}$ similar to the dijet balancing. These events are also used to cross check the jet energy corrections, but they are not as useful for deriving the corrections as the agreement in the balance between signal and background $\gamma$-jet events depends strongly on the choice of selection requirements. The systematic uncertainty on the absolute correction is driven by the uncertainty on the simulation of calorimeter response to hadrons. It also includes the uncertainty on the simulated calorimeter response to electromagnetic particles, the stability of calorimeter response over time, and differences in jet fragmentation models used by the PyTHIA [54] and HERWIG [55] MC generators [40]. 
After these corrections are applied, we require the jet $|\eta|$ to be less than 2.0 to avoid misreconstructing jets outside the instrumented region of the CDF detector. We also require that the corrected jet $E_{T}$ be greater than $20 \mathrm{GeV}$. These corrected jets have been mapped only to the particle representation of the initial hadronized quark, therefore, we must develop another mapping function to account for the difference in quark energy and the energy of the particles after hadronization. This mapping method is not necessary for the selection of jets and is only relevant to the top quark mass measurement. It will be described later in Sec. 6.4

Although we do not apply any additional corrections to the jets, we do calculate two more energy corrections as a result of extra energy from the spectator partons in the $p \bar{p}$ interaction (known as underlying event) and radiation leaking outside of the jet cone in the calorimeter (out-of-cone and splash-out) [40. The uncertainties from the corrections along with those from the previously detailed corrections are considered in the systematic uncertainties of the measurements. The out-of-cone and underlying event corrections are calculated together in much the same way as the absolute correction. Using PYTHIA dijet samples with the jets matched to partons, we derive new mapping functions to map the particle jet to the actual parton. The out-of-cone uncertainty is derived by studying $\gamma$-jet events. We compare the difference in the energy measured in the calorimeter outside of the jet cone as a function of the jet $P_{T}$ for data and MC events where the $P_{T}$ of the $\gamma$ is assumed to be the correct jet $P_{T}$. This is done with both Pythia and Herwig MC, and the uncertainty is taken to be the largest difference between the effect in either PythiA or HeRwig MC and data. Effects from the underlying event are already accounted for to first order in the MC generators. To derive a systematic uncertainty for these corrections, we compare the momentum of charged tracks which are transverse (between 60 degrees and 90 degrees) to the lead jet in the $\mathrm{r}-\phi$ plane in data and MC events. Finally, the out-of-cone uncertainty only considers energy leakage outside of the jet cone to a cone of radius 1.3. PYTHIA MC samples show an additional $0.5 \mathrm{GeV}$ leaks outside this cone. We take half of that value as an additional uncertainty called splash out. Each individual jet correction uncertainty along with the total sum in quadrature of all uncertainties is shown as a 
function of jet $P_{T}$ in Fig. 3.2 [40].

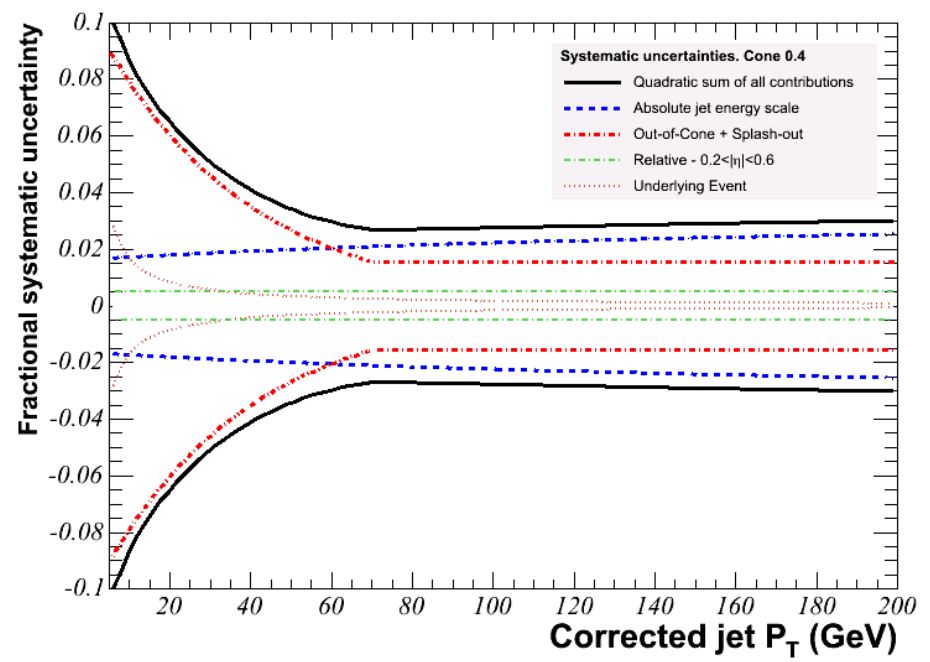

Figure 3.2: Fractional uncertainties from the various jet corrections as a function of jet $P_{T}$. The black line shows the sum in quadrature of all uncertainties. The uncertainty tends to be $3 \%$ for high energy jets but can be as large as $7 \%$ for lower energy jets which pass the selection requirement [40, 56].

\section{Heavy Flavor Jets and b-tagging}

The long lifetime of B hadrons gives us a unique opportunity to identify jets originating from $b$ quarks. Because the $t \bar{t}$ decay always produces two $b$ quarks, by requiring events to have at least one jet identified (or b-tagged) as coming from a $b$ quark allows us to significantly reduce the contribution from background processes which are less likely to produce $b$ quarks such as $W+$ jets events and QCD multijet production. When a $b$ quark is produced from a top quark decay, it is highly boosted due to the large mass difference between the top and $b$ quark, and it hadronizes almost immediately (on the order of $10^{-24} \mathrm{~s}$ ) to a $\mathrm{B}$ hadron. This B hadron has an average lifetime of 1 to 2 ps. As a result, the $\mathrm{B}$ hadron traverses a few $\mathrm{mm}$ in the detector before decaying into a jet of charged and neutral particles which produces a secondary vertex displaced from the primary vertex in the $\mathrm{r}-\phi$ plane.

At CDF, the secondary vertex detection algorithm (SecVtx) [57] [58] looks for these 
vertices with information from the high precision tracking from the silicon detector. This algorithm examines each jet for tracks with large impact parameters in relation to the primary vertex and attempts to reconstruct them into a secondary vertex. Once a secondary vertex is identified, the 2 dimensional distance in the $\mathrm{r}-\phi$ plane between the primary and secondary vertex $\left(L_{x y}\right)$ is calculated. If $L_{x y}$ is $>7.5$ times the uncertainty on $L_{x y}$ of approximately $190 \mu \mathrm{m}$, then the jet is considered to be "tagged" meaning it has a secondary vertex. Finally, the algorithm checks the dot product between the vector from the primary vertex to the secondary vertex and the jet momentum. If this is positive, in which case the jet is traveling away from the primary vertex, the event is said to have a positive b-tag, and the jet is considered to be coming from a $b$ quark. If the dot product is negative, the jet is traveling from the secondary vertex back toward the primary vertex and the event is said to have a negative b-tag. Negative b-tag events are not kinematically possible which means that the secondary vertex is created by resolution effects in the CDF tracking system. These negative b-tag events provide a background estimate to the number of incorrectly tagged positive tag events. Graphical examples of the b-tagging algorithm and positive and negative tags are shown in Fig. 3.3. The efficiency for SecVtx tagging for jets from $b$ quarks in top quark decays are shown in Fig. 3.4 as a function of jet $P_{T}$ and $|\eta|$ [59]. Assuming the tagging efficiency is $40 \%$, a simple probability calculation shows that $65 \%$ of $t \bar{t}$ decays with $2 b$ quarks will have at least one jet with a b-tag.

\subsubsection{Neutrinos}

Neutrinos ( $\nu$ 's) are nearly massless, charge neutral leptons in the SM. They come in three flavors: electron, $\mu$, and $\tau$. They interact only through the weak force and have very small interaction cross sections. This means $\nu$ 's generally travel through large quantities of matter without ever interacting. As a result, we cannot directly identify $\nu$ 's in the CDF detector. Instead, we look for evidence of $\nu$ 's by looking for an imbalance in the $E_{T}$ of an event. If we assume that the colliding $p$ and $\bar{p}$ have no transverse momentum, then by conservation of momentum, the final state of any interaction event should also have no transverse momentum. Although the quarks and gluons in the $p$ and 

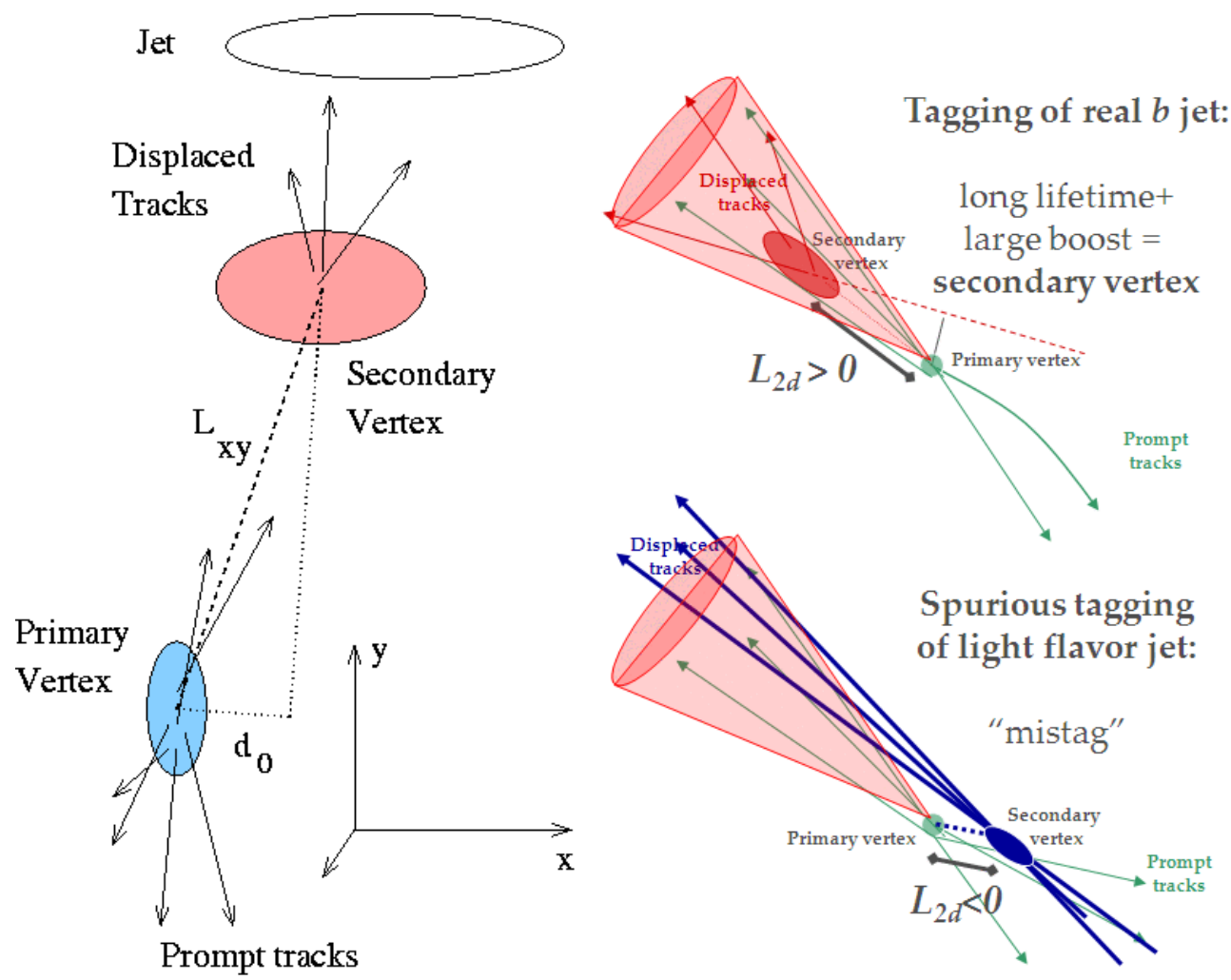

Figure 3.3: On the left is a diagram showing the identification of a secondary vertex and the definition of the impact parameter $d_{0}$ and $2 \mathrm{D}$ distance $L_{x y}$. On the right is shown positive tagging (top) for a real jet from a $b$ quark and negative tagging (bottom) or the mistag of a jet from a light flavor quark [60].
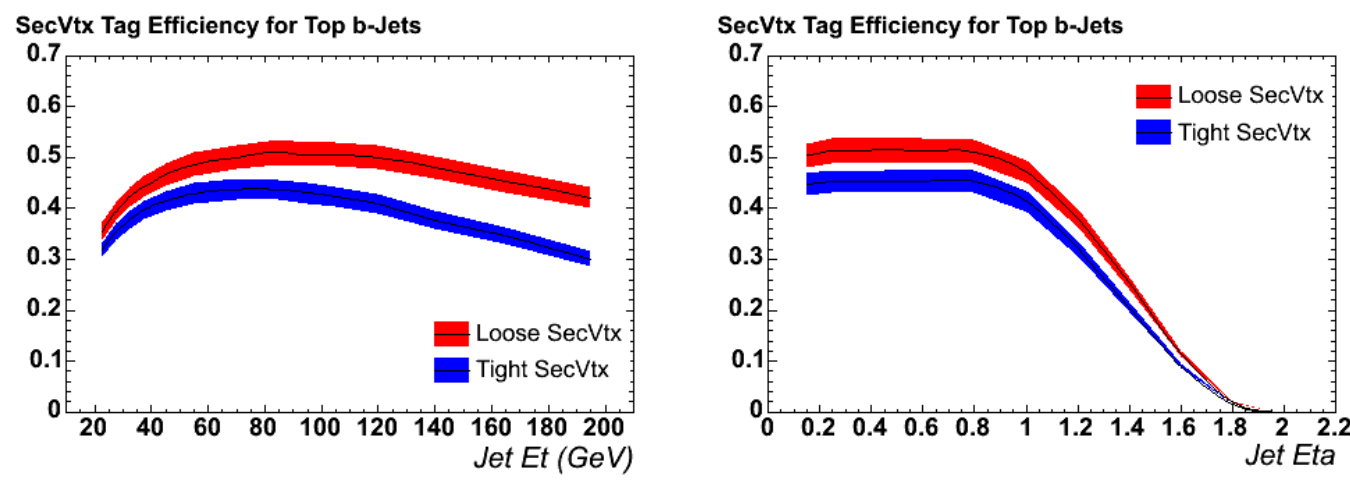

Figure 3.4: The SecVtx tagging efficiency for a $b$ jet from a top quark decay as a function of $E_{T}$ (left) and $|\eta|$ (right). For the sake of this analysis, we only consider the tight SecVtx algorithm [59]. Assuming the efficiency is $\sim 40 \%$, simple probability predicts that $65 \%$ of $t \bar{t}$ decays with two $b$ quarks will have at least one b-tagged jet. 
$\bar{p}$ which interact with each other may have some small net transverse momentum, this assumption is generally valid. With this in mind, we measure the imbalance through a variable called missing $E_{T}\left(E_{\mathrm{T}}\right)$ :

$$
\overrightarrow{E_{\mathrm{T}}}=-\sum_{i}^{\text {towers }} E_{T i} n_{\hat{T} i}
$$

where the sum is taken over all the towers in the calorimeter, $E_{T i}$ is the uncorrected $E_{T}$ in the $i^{t h}$ tower, and $n_{T i}$ is the unit vector in the x-y plane pointing to the $i^{t h}$ calorimeter tower from the primary vertex of the event. The $E_{\mathrm{T}}$ is also corrected for $\mu$ energy since $\mu$ 's are minimum ionizing particles which deposit very little energy in the calorimeter and for the jet energy corrections described in Sec. 3.2 .2 . It is important to note that the $E_{\mathrm{T}}$ only accounts for the transverse components of the $\nu$ momentum. We cannot make any assumptions about the initial state's momentum in the $\mathrm{z}$ direction, so we cannot use a similar method to estimate the $\mathrm{z}$ component of a $\nu$ 's momentum. Additionally, if an event has more than $1 \nu$, the $E_{\mathrm{T}}$ only gives the vector sum of the multiple $\nu$ 's transverse momentum. For this analysis, we require that events have a $E_{\mathrm{T}} \geq 20 \mathrm{GeV}$.

\subsubsection{Lepton Veto}

In $t \bar{t} \rightarrow \tau+$ jets decays, the $\tau$ is the only lepton in the final state, therefore, we veto events with an identified electron or $\mu$. The lepton identification is only used as a veto, so we do not require the electron and $\mu$ identification to be quite as stringent as we would for signal selection.

An electron is an electrically charged particle subject to the electroweak force. Its detector signature is a single isolated track pointing to an energy cluster in the electromagnetic calorimeter. The energy cluster comes from an electromagnetic shower of Bremsstrahlung radiation and pair production of $e^{-} e^{+}$from radiated $\gamma$ 's within the electromagnetic calorimeter. The electron energy is measured from at most 2 towers in the CEM calorimeter. To be identified, electrons must fall within the central region of the detector $|\eta|<1.0$ which is covered by the CEM calorimeter and must pass fiduciality requirements that ensures the electron shower is measured by the CES detector 


\begin{tabular}{|c|c|}
\hline Variable & \\
\hline Region & CEM \\
\hline Fiducial & $==1$ \\
\hline$E_{t}$ & $\geq 20 \mathrm{GeV}$ \\
\hline $\mid$ Track $Z_{0} \mid$ & $<60 \mathrm{~cm}$ \\
\hline Track $P_{t}$ & $>10 \mathrm{GeV}$ \\
\hline COT Ax. Seg & $\geq 3$ \\
\hline COT St. Seg & $\geq 2$ \\
\hline Conversion & $\neq 1$ \\
\hline $\mathrm{Had} / \mathrm{Em}$ & $\leq(0.055+(0.00045 \times E))$ \\
\hline$E_{t}^{i s o l} / P_{t}$ & $<0.1$ \\
\hline
\end{tabular}

Table 3.2: Electron identification requirements

subsystem. The track must have a $P_{T}$ of at least $10 \mathrm{GeV}$, originate within $60 \mathrm{~cm}$ of the center of the CDF detector $\left(\left|Z_{0}\right|<60 \mathrm{~cm}\right)$, and have at least 5 hits in three or more axial and two or more stereo segments of the COT. The electron $E_{T}$ is required to be $20 \mathrm{GeV}$ or greater. Electrons should shower most of their energy in the electromagnetic calorimeter rather than the hadronic calorimeter, so we require that the ratio of EM to hadronic energy be less than or equal to $0.055+(0.00045 * E)$, where $E$ is the candidate electron's energy. Additionally, since we do not want to mistake secondary electrons from photon conversions for prompt electrons from a $W$ decay, we apply a conversion tag to the electrons which essentially looks for an oppositely charged partner track originating from a similar conversion point as the original track [61]. Finally, we require that the electron's energy cluster be isolated in the detector to reject $\tau$ 's or jets with high EM fractions which may otherwise pass the electron selection requirements. To check for isolation, we calculate the calorimeter energy within a $0.4 \eta-\phi$ cone around the electron cluster minus the energy of the electron cluster. If the ratio of this energy to the electron cluster energy is less than $10 \%$, we are satisfied that the electron is well isolated in the detector. A summary of these selection requirements is given in Tab. 3.2

The $\mu$ behaves similarly to an electron except due to its higher mass, it emits very little bremsstrahlung radiation. As a result, $\mu$ 's pass through the calorimeter with minimal energy deposit and are not stopped by the calorimeter. Their decay signature 


\begin{tabular}{|l|c|}
\hline Variable & $20 \mathrm{GeV}$ \\
\hline$P_{t}$ & $2+\max (0,0.0115 \times(p-100)) \mathrm{GeV}$ \\
EM Energy & $6+\max (0,0.028 \times(p-100)) \mathrm{GeV}$ \\
Had Energy & $<0.1$ \\
$E_{t}^{\text {isol }} / P_{t}$ & $\geq 3($ unless $\mu$ in BMU $)$ \\
COT Ax. Seg & $\geq 2($ unless $\mu$ in BMU $)$ \\
COT St. Seg & $\leq 60 \mathrm{~cm}$ \\
$\mid$ Track $Z_{0} \mid$ & $<0.02 \mathrm{~cm} \mathrm{w} / \mathrm{si},<0.2 \mathrm{~cm} \mathrm{w} / \mathrm{o} \mathrm{si}$ \\
$\left|d_{0}\right|$ & \\
\hline
\end{tabular}

Table 3.3: Muon identification requirements

is a single isolated track with minimal energy deposits in the calorimeter and a track stub in one of the $\mu$ tracking chambers located on the outside of the CDF detector. For the sake of $\mu$ rejection, we even consider muons which pass all selection requirements but fail to be associated with a hit in the $\mu$ systems. The requirements listed here are summarized in Tab. 3.3. To be identified, the $\mu$ must have a $P_{T}$ of at least $20 \mathrm{GeV}$. We place maxima on the EM and hadronic calorimeter energies of the $\mu$. The EM energy must be no greater than $2 \mathrm{GeV}+M A X\left(0,0.0115 \times\left(P_{\mu}-100 \mathrm{GeV}\right)\right)$ where $P_{\mu}$ is the momentum of the $\mu$ in $\mathrm{GeV}$ and $M A X$ refers to taking the greater of the two options. The hadronic energy must be no greater than $6 \mathrm{GeV}+M A X\left(0,0.028 \times\left(P_{\mu}-100 \mathrm{GeV}\right)\right)$. The track must have an original $\mathrm{z}$ vertex position $\left(Z_{0}\right)$ with $60 \mathrm{~cm}$ of the center of the $\mathrm{CDF}$ detector and its impact parameter with regard to the beamline $\left(d_{0}\right)$ must be less than $0.2 \mathrm{~cm}$ if the track has no silicon hits and $0.02 \mathrm{~cm}$ if the track has hits in the silicon detector. Its track must also have at least 5 hits in 3 or more axial and 2 or more stereo segments of the COT. This requirements is removed for BMU muons as their tracks will likely not pass through the full COT radius. Finally, the $\mu$ must pass the same isolation requirement as the electron. Its calorimeter energy within a 0.4 cone must be less than $10 \%$ of its $P_{T}$.

\subsection{Event Selection Requirements}

So far, we have described the decay signature and selection requirements for the various particles of interest to this analysis. Examples of how these particles appear in the CDF 
detector can be seen in Fig. 3.5 .

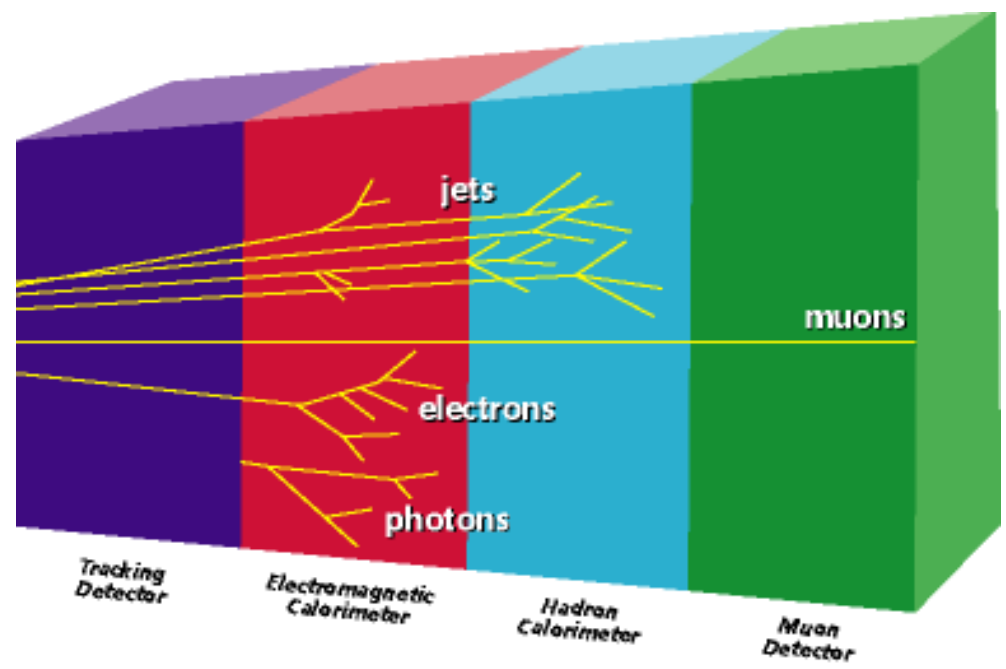

Figure 3.5: Representations of the CDF detector signatures for various common particles 62 .

The final state of the $t \bar{t} \rightarrow \tau+$ jets decay gives 2 jets from $b$ quarks, 2 jets typically from light flavor quarks from a $W$ decay, a single narrow jet from the hadronic $\tau$ lepton decay, and two $\tau$ flavor $\nu$ 's. To select these events, we apply selection requirements which require 4 jets with $\mathrm{E}_{T}>20 \mathrm{GeV}$ (as described in Sec. 3.2.2. $E_{\mathrm{T}}>20 \mathrm{GeV}$ (as described in Sec. 3.2.3), and a single hadronic $\tau$ lepton with the selection requirements listed in Tab. 3.1. Additionally, one of the 4 jets must be identified as coming from a $b$ quark as described in Sec. 3.2.2. Since the signal process only gives a single $\tau$ lepton, we also veto any event which contains an identified electron or $\mu$ with the requirements listed in Tab. 3.2 and Tab. 3.3 respectively. With these requirements, we select a total of 162 candidate $\tau+$ jets events. 


\section{Chapter 4}

\section{Background Models and Estimation}

Several processes have signatures which either are identical to or mimic the hadronic $\tau+$ jets final state. This chapter describes these background processes which can pass the event selection and the methods we use to simulate, reduce, and estimate them. These processes either include a $W$ or $Z$ boson which decays to a hadronic $\tau$ and other particles or have a jet which fakes the signature of a hadronic $\tau$. Additionally, since hadronically decaying $\tau$ 's appear to be narrow jets, any hadronic $\tau$ which does not pass the $\tau$ identification selection requirements results in a fake jet, assuming it is energetic enough to pass the jet selection requirements. The background processes that contribute to this analysis, in order of importance, are QCD multijet productions, $W+$ jets, $Z+$ jets, electroweak diboson production ( $W W, W Z$, and $Z Z$ production), and single top production in either the s or t channel.

Due to the high QCD multijet cross section at hadron colliders and the similarities between the $\tau+$ jet and QCD multijet signals, we find QCD multijet production events dominate the data sample gathered with the standard event selection. To reduce the contribution of QCD multijet events, we introduce a neural network (NN) which is trained to distinguish between $t \bar{t} \rightarrow \tau+$ jets events and QCD multijet events. We will use a data-driven approach to estimate the contribution from the various background sources. This approach predicts the contribution from QCD multijet and $W+$ jets events by fitting the shapes of the NN output distribution for the various signal and background processes to the distribution seen in the data. We optimize the choice of the NN selection value by maximizing the signal significance as predicted by the background estimate, and finally, we produce the estimated contribution from $t \bar{t} \rightarrow \tau$ + jets events and each background contribution. 


\subsection{Monte Carlo Samples}

We generate $t \bar{t}$, diboson, and single top events with the PYтнia [54] Monte Carlo (MC) generator. For all MC in this analysis, the $\tau$ lepton decay is handled by the TAUOLA package [63] which properly accounts for the polarization of the $\tau$. For $t \bar{t} \mathrm{MC}$, we use 21 different samples ranging in mass from 155 to $195 \mathrm{GeV}$. For the entire range of 155 to $195 \mathrm{GeV}$, we use 9 samples produced at a step size of $5 \mathrm{GeV}$. From 165 to $185 \mathrm{GeV}$, we include samples with a step size of $2.5 \mathrm{GeV}$ (adding samples with top quark mass of $167.5,172.5$, etc.). Finally, in the expected central region of 170 to $180 \mathrm{GeV}$, we chose all samples with a step size of $1 \mathrm{GeV}$.

The $W$ and $Z+$ jets backgrounds are simulated with Alpgen [64] MC generator, using Pythia [54] for the hadronization of partons. Both $W$ and $Z+$ jets samples are broken down into contributions from light and heavy flavor. The AlPGEN generator produces simulated events from a theoretical computation of the matrix elements for $W$ or $Z+b b, c c$, or $c+\mathrm{N}$ partons for heavy flavor contributions. For light flavor contributions, a matrix element for $W$ or $Z+\mathrm{N}$ light flavor partons is used. These events then are run through PYTHIA to develop the particle showers from the hadronization of partons. As showering can give rise to heavy flavor partons, jets in the MC events are matched to partons and an overlap removal scheme [65] 66] is used to remove duplicate heavy flavor contributions from matrix element creation and showering effects. All simulated events are run though the CDF detector simulation based on the GEANT3 package [67].

For the QCD multijet background, we use a data based model which will be described in the next section.

\subsection{Data Driven QCD Multijet Estimate}

Due to the difficulty in MC modeling of QCD multijets events, we use data to model these events. This model is particularly important to this analysis because of the high QCD multijet production cross section at the Tevatron. Since QCD multijet events do not produce prompt leptons (leptons produced from the initial interaction) or $\nu$ 's, 
QCD multijet events can generally be vetoed by selecting events with large $E_{\mathrm{T}}$ and leptons which are isolated in the detector's $\eta-\phi$ space (leptons can be produced in the hadronization of jets from QCD multijets, but these leptons appear within the jet cone). However, because $\tau$ 's appear in the CDF detector as narrow jets, QCD multijet events with moderate $E_{\mathrm{T}}$ due to detector energy resolution effects can fake a $\tau+$ jets signal at a substantial rate. Due to this high rate and the large QCD multijet cross section at the Tevatron, we find that QCD multijet production is the dominant background process to $t \bar{t} \rightarrow \tau+$ jets events.

We exploit the similarity between $\tau$ 's and jets to easily define a "fake" $\tau$ candidate to use in modeling the selected QCD multijet events. Since jets tend to not be as narrow as $\tau$ 's, we define "fake" $\tau$ 's as $\tau$ candidates which pass all $\tau$ selection requirements (see Tab. 3.1) except for the track isolation. By requiring that the $\tau$ candidate has at least 1 track in the $\tau$ isolation annulus, we ensure that no single event will be included in both the signal selection and QCD multijet model. Additionally, since we use the same trigger and otherwise identical selection requirements, we avoid introducing any trigger bias into this model. By studying the acceptance of $t \bar{t}$ events with the QCD model selection requirements, we find the $t \bar{t}$ contamination in the fake $\tau+$ jets events is less than $10 \%$.

We test how well this model matches the actual QCD multijet events by selecting data with the standard event selection with certain requirements removed. By removing the $E_{\mathrm{T}}$ and one or more b-tag requirements, we find the selected data is completed dominated by QCD multijet production due to the high QCD multijet cross section. We then compare kinematic distributions between the events from this "QCD multijet enriched" data and events from the QCD multijet model (See Fig. 4.1). We find that the model matches the QCD enriched data extremely well, and the uncertainty is based on the statistical limit from the number of events selected in the QCD multijet model.

With the $E_{\mathrm{T}}$ and b-tagging requirement in the standard event selection, we estimate that roughly $75 \%$ of the 162 selected candidate $t \bar{t} \rightarrow \tau+$ jets events are from QCD multijet production rather than signal. To improve the signal purity of the events, we introduce a NN to identify and remove QCD multijet events. 

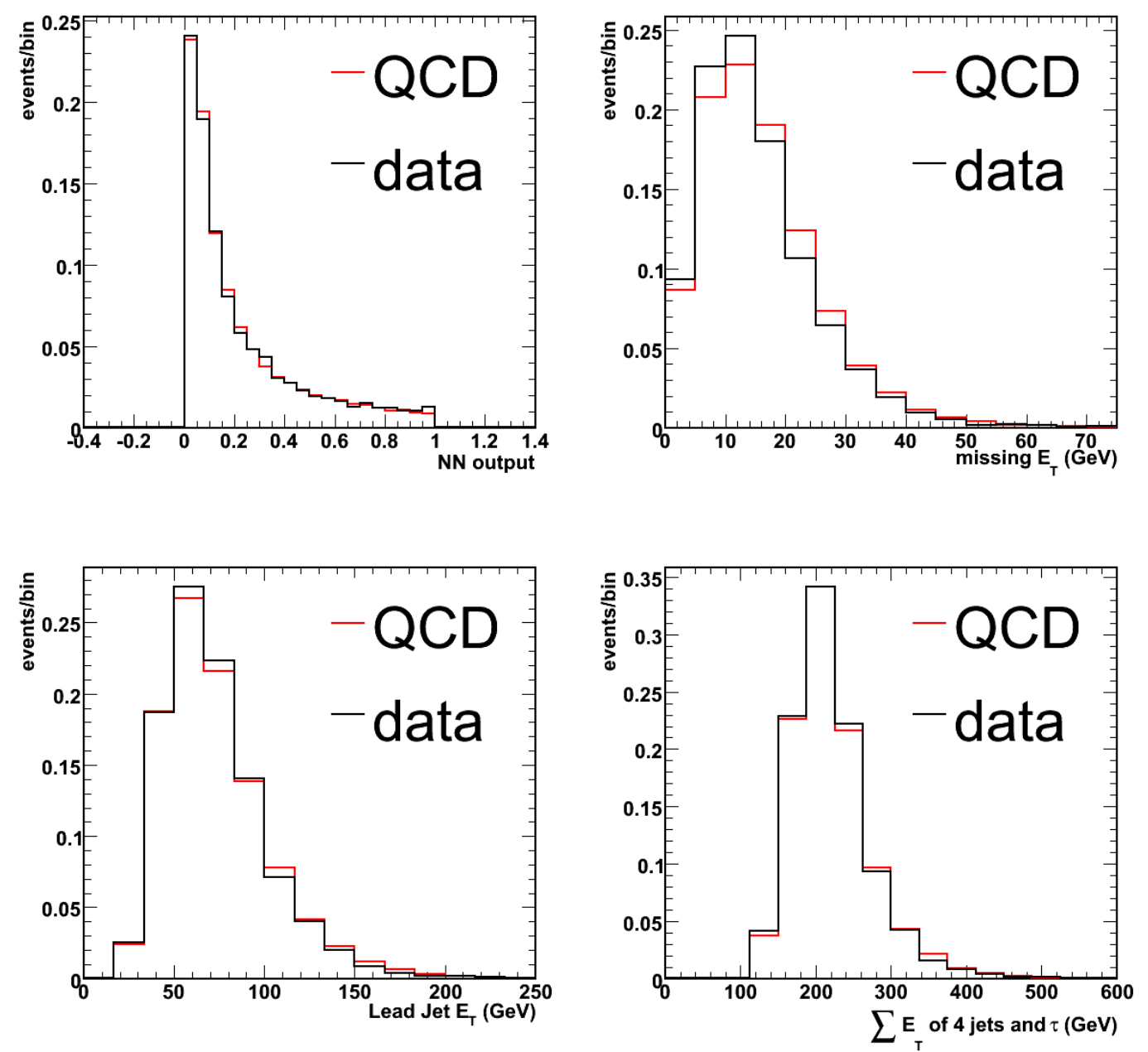

Figure 4.1: Comparison distributions for "QCD multijet enriched" data and the QCD multijet selection. The data is selected with the standard requirements except the $E_{\mathrm{T}}$ and b-tagging requirements are removed. The NN output (upper left), $E_{\mathrm{T}}$ (upper right), lead jet $E_{T}$ (lower left), and $\sum E_{T}$ of the jets and $\tau$ (lower right) are shown. All distributions are normalize to 1 . The agreement in the distributions shows that the QCD multijet model is properly modeling the QCD multijet events in the data. 


\subsubsection{Neural Network for Reduction of QCD Multijet Background}

To improve the QCD multijet rejection, we introduce a Neural Network (NN) designed to distinguish QCD multijet events from $t \bar{t} \rightarrow \tau+$ jets. We use a MultiLayerPerceptron [68] network through the TMultiLayerPerceptron package in Root [69]. The NN is made up of nodes in multiple layers. The first layer contains one node for each input variables. Each node in this layer accepts one input variable, normalizes it, and passes it on to the nodes in the next layer. The last layer has a single node which reads out a value from 0 to 1 . In between the input and output layers can be any number of "hidden layers" each with as many nodes as we desire to use. Generally, the NN performance does not show significant improvement as more hidden layers are added, so we limit ourselves to considering NN's with 1 or 2 hidden layers. Each node in a hidden layer forms a linear combination of the output of the nodes in the previous layer based on a series of weights linking the node to the nodes in the previous layer. The node then computes an output value from a sigmoid function of the input linear combination. The final node in the output layer differs in that its output is computed as a linear function of the input. If the $\mathrm{NN}$ is trained with signal events expecting an output of 1 and background events expecting an output of 0 , the final node returns the probability that the event in question is a signal event based on the input variables.

Generally speaking, the NN is trained on signal and background events with expected output of 1 and 0 , respectively. Training and test samples are built with equivalent numbers of signal and background events distributed randomly throughout the sample. As the $\mathrm{NN}$ is trained, it chooses weights at each training iteration (called an epoch) which minimize the error on the training sample. The error is computed based on the difference between the expected output of 0 or 1 and the computed output from the final node for each event. We chose to train the NN with the Broyden-FletcherGoldfarb-Shanno (BFGS) training method [71] which is a quasi-Newtonian method for finding the global minimum for the NN error in the parameter space of the NN weights. At each epoch, the error from the test sample is also computed, but it is not used to guide the training algorithm. As the $\mathrm{NN}$ training iterates over epochs, the error on the 


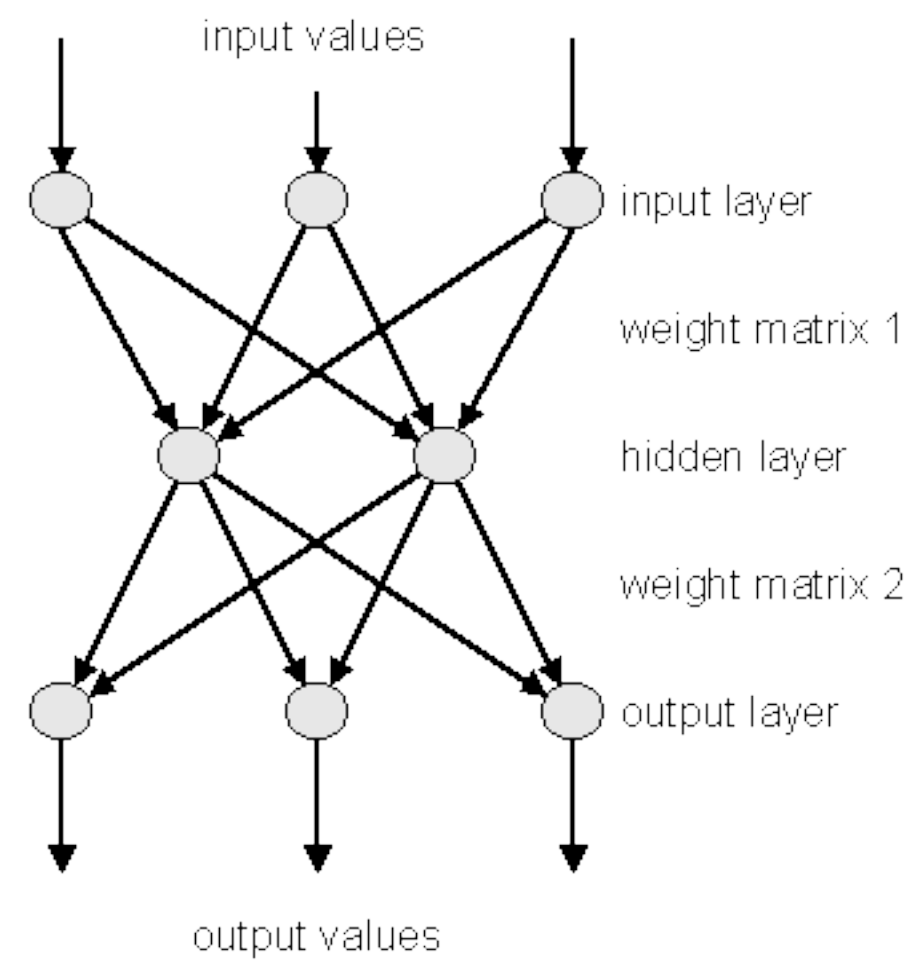

Figure 4.2: Example structure of a MultiLayerPerceptron neural network [70].

training sample decreases as the NN finds the ideal set of weights to differentiate the signal and background samples. The error on the test sample also decreases to a point. Eventually, either the error on these two samples will fail to decrease further, indicating that the NN cannot find any new set of weights which improves the performance or the training sample error continues to decrease while the test sample error begins to increase, indicating that the $\mathrm{NN}$ is "over-trained." An over-trained network is one that begins to separate signal and background events based on details in the training sample which are not indicative of the actual distribution of events.

We considered several input variables based on the differences between QCD multijet events and $t \bar{t} \rightarrow \tau+$ jets events. For example, as QCD multijets events do not explicitly involve $\nu$ 's, we find the distribution of $\mathbb{E}_{\mathrm{T}}$ to be lower in QCD multijet events. Additionally, jets from QCD multijet events tend to be softer than jets from top quark decay. Finally, since QCD multijet events often do not include a $W$ boson, we find a difference in the transverse mass calculated from the $\tau$ and $E_{\mathrm{T}}$ from $t \bar{t}$ decays with true 
$W$ bosons. We use all these features to select the variables on which to train the NN. We considered several NN setups with different input variables and NN structures. We choose the version that gives the best separation between the signal and background samples (as shown in Fig. 4.7) and the lowest total error for the NN output of input signal $(t \bar{t} \rightarrow \tau+$ jets) and background (QCD multijet) events. We find the best performance comes from a NN with 8 input variables and 2 hidden layers of 10 and 4 nodes respectively. The 8 input variables used are:

- $E_{\mathrm{T}}$

- Scalar $\sum E_{T}$ of the tight jets and $\tau$

- Scalar $\sum E_{T}$ of the two highest $E_{T}$ jets

- Scalar $\sum E_{T}$ of the two lowest $E_{T}$ jets and the $\tau$

- Transverse Mass, defined as $M_{\text {trans }}=\sqrt{E_{T}^{2}-P_{T}^{2}}$, of the leptonically decaying $W$

- Lead Jet $E_{T}$

- Average $\eta$ Moment of all non-btagged jets ( $\eta$ moment for a jet is defined as: $\left.<\eta>=\sqrt{\sum_{\text {towers }}\left[\frac{E_{T}^{\text {tower }}}{E_{T}^{\text {jet }}}\left(\eta_{\text {tower }}-\eta_{\text {jet }}\right)^{2}\right]}\right)$

- Lowest Dalitz Variable (defined below)

For any given triplet of jets $\mathrm{i}, \mathrm{j}$, and $\mathrm{k}$, the Dalitz variables (D) are dimensionless variables defined as:

$$
D=\frac{M_{i j}^{2}}{M_{i j k}^{2}+M_{i}^{2}+M_{j}^{2}+M_{k}^{2}}
$$

By this definition, there are three Dalitz variables for each jet triplet, each with one of the three numerators $M_{12}, M_{13}$, and $M_{23}$ and the same denominator. For any jet triplet, the three variables will sum to 1 . We order these Dalitz variables from largest to smallest as $D_{\text {large }}, D_{\text {med }}$, and $D_{\text {small }}$. We then make a multiple entry distribution by overlaying $D_{\text {large }}$ vs. $D_{m e d}, D_{\text {large }}$ vs. $D_{\text {small }}$, and $D_{m e d}$ vs. $D_{\text {small }}$. In this distribution, for $t \bar{t}$ decays the $W$ resonance inside the top resonance will be apparent at a value of $\frac{M_{W}^{2}}{M_{\text {top }}^{2}}$ as can be seen in the left distribution in Fig. 4.3 . Additionally, since QCD multijet 
events do not contain any resonance, these events tend to pile up along the lower edges of the Dalitz distributions as can be seen in the right distribution in Fig. 4.3. The selected events have 4 jets, so there are 4 independent triplets $(123,124,134$, and 234) which give 12 different Dalitz variables for each event. We find that the smallest of the $4 D_{\text {small }}$ possible Dalitz variables from each independent jet combination helps the NN to distinguish QCD multijet and $t \bar{t}$ events.
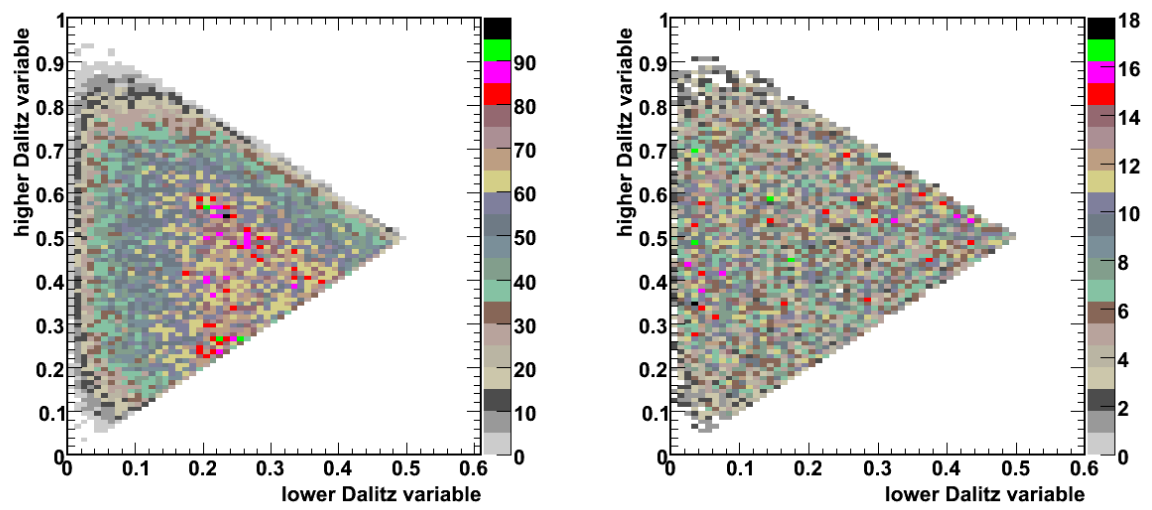

Figure 4.3: Multiple entry distributions of higher vs lower Dalitz variables for $t \bar{t}$ events (left) and QCD multijet events (right). The $W$ resonance within the top resonance creates the triangular shaped pileup in the left distribution.

Normalized distributions of the $t \bar{t}$ and QCD multijet events for each variable used in the NN are shown in Figs. 4.4 and 4.5. The separation between the red line for QCD multijet events and the black line for $\mathrm{MC} t \bar{t}$ events with $M_{\text {top }}=172.5 \mathrm{GeV}$ show the power of these variables to help distinguish $t \bar{t}$ events from QCD multijet events. Additionally, the dashed lines show the same distributions from MC $t \bar{t}$ events with $M_{\text {top }}=165 \mathrm{GeV}$ and $185 \mathrm{GeV}$. Since these distributions do not differ greatly from the nominal distribution with $M_{t o p}=172.5 \mathrm{GeV}$, we conclude that the NN output will not have a strong dependence on the mass of the top quark.

We build training and test samples for the NN using the standard $\tau+$ jets and QCD multijet event selection described above; however, to improve the statistics of QCD multijet sample and to make sure we do not bias the input variables of the NN, we remove the $E_{\mathrm{T}}$ and b-tagging requirements. The $\tau+$ jets sample comes from events 

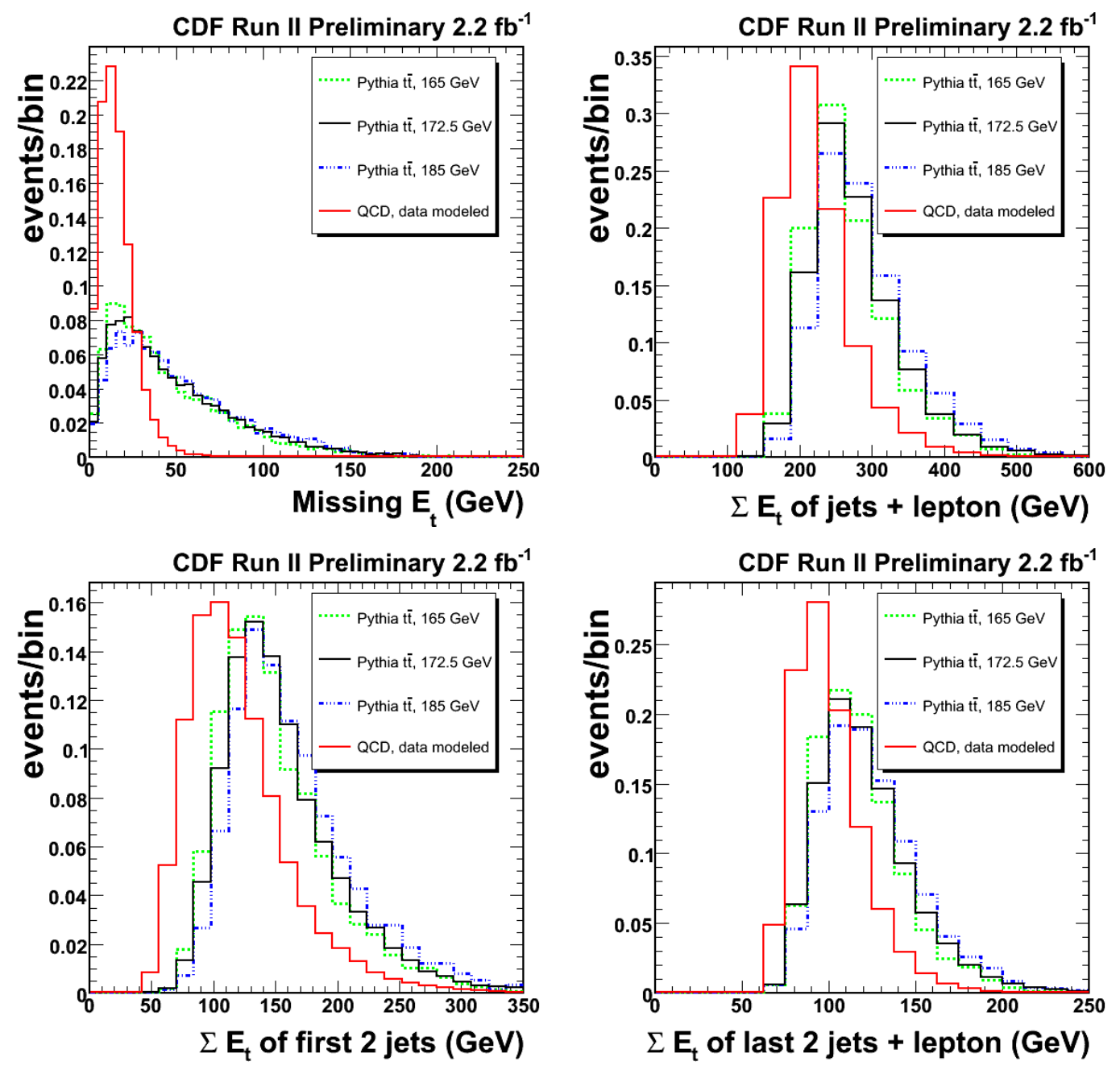

Figure 4.4: The $t \bar{t}$ and QCD multijet distributions for the first four variables used in the NN. Each distribution is normalized to 1 so that they can be shown on the same axis. The red line is the distribution from QCD multijet events, and the black line is from Pythia MC $t \bar{t}$ events with $M_{t o p}=172.5 \mathrm{GeV}$. These variables show good separation between the QCD multijet and $t \bar{t} \rightarrow \tau+$ jets events. Additionally, the dashed lines show the distributions from Pyтнia MC $t \bar{t}$ events with $M_{t o p}=165$ and $185 \mathrm{GeV}$. Comparing these distributions to the nominal distribution with $M_{t o p}=172.5 \mathrm{GeV}$ shows that the NN output does not have a large dependence on $M_{\text {top }}$. 

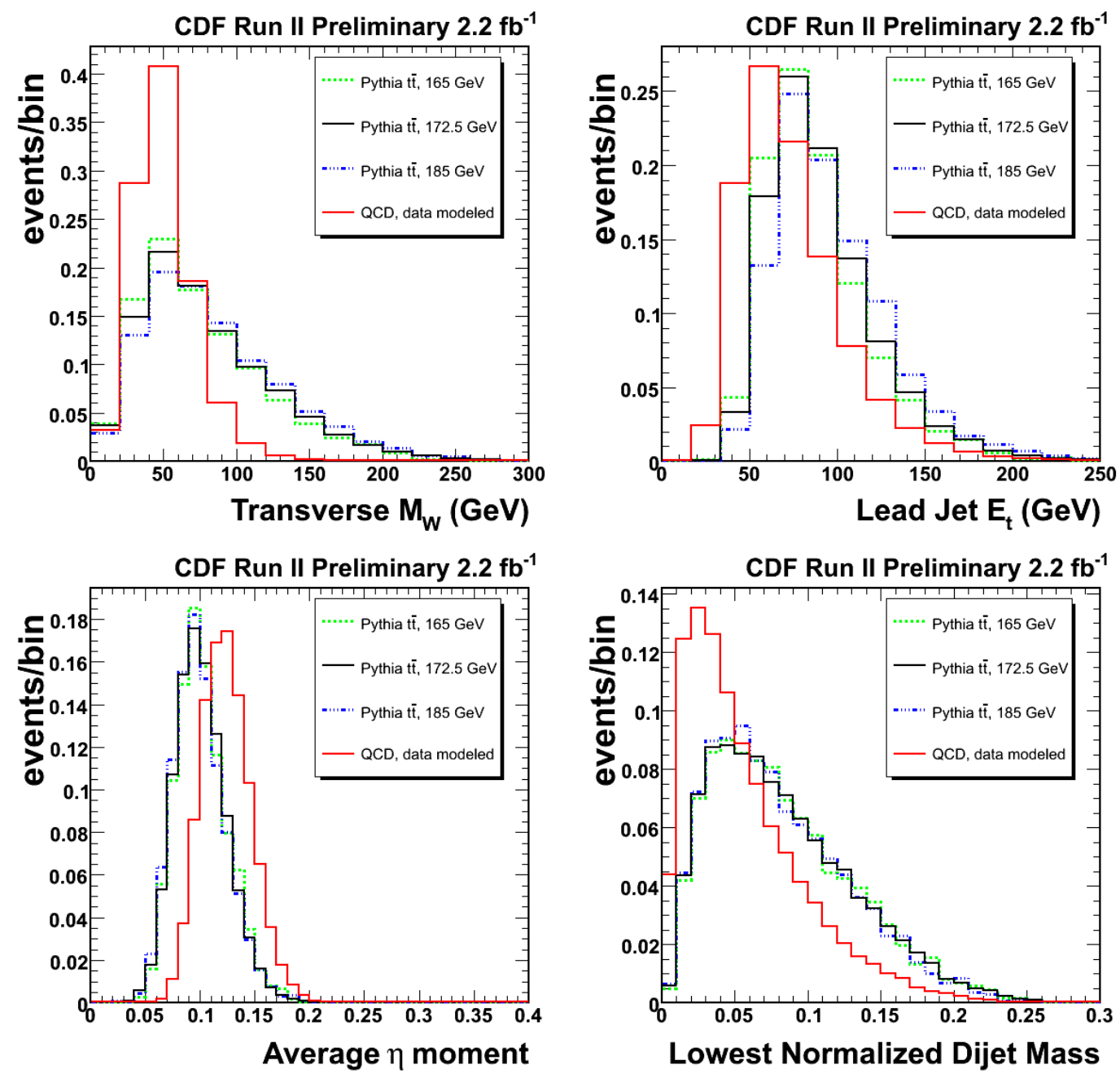

Figure 4.5: The $t \bar{t}$ and QCD multijet distributions for the second four variables used in the NN. Each distribution is normalized to 1 so that they can be shown on the same axis. The red line is the distribution from QCD multijet events, and the black line is from Pyтнia MC $t \bar{t}$ events with $M_{t o p}=172.5 \mathrm{GeV}$. These variables show good separation between the QCD multijet and $t \bar{t} \rightarrow \tau+$ jets events. Additionally, the dashed lines show the distributions from Pythia MC $t \bar{t}$ events with $M_{t o p}=165$ and $185 \mathrm{GeV}$. Comparing these distributions to the nominal distribution with $M_{t o p}=172.5 \mathrm{GeV}$ shows that the NN output does not have a large dependence on $M_{t o p}$. 
selected from $t \bar{t} \mathrm{MC}$ with a top quark mass of $172.5 \mathrm{GeV}$, and QCD multijet events were selected from data as described above. Both training and test samples are built with equal numbers of signal and background events. Due to the total number of selected signal and background events we have to work with, each sample has approximately 18000 events. Despite attempts to over-train the NN, as more epochs are added to the training, the NN eventually fails to find any further improvement, as shown in Fig. 4.6. The output distribution after training the $\mathrm{NN}$ is shown in Fig. 4.7. The clear twin peaks in this distribution shows that the $\mathrm{NN}$ is giving excellent separation between $t \bar{t}$ and QCD multijet events. Finally, we optimize the QCD multijet rejection by throwing out all events with NN output less than 0.85 . The motivation for the selection of 0.85 is described along with the Background Estimate in Sec. 4.2.2.

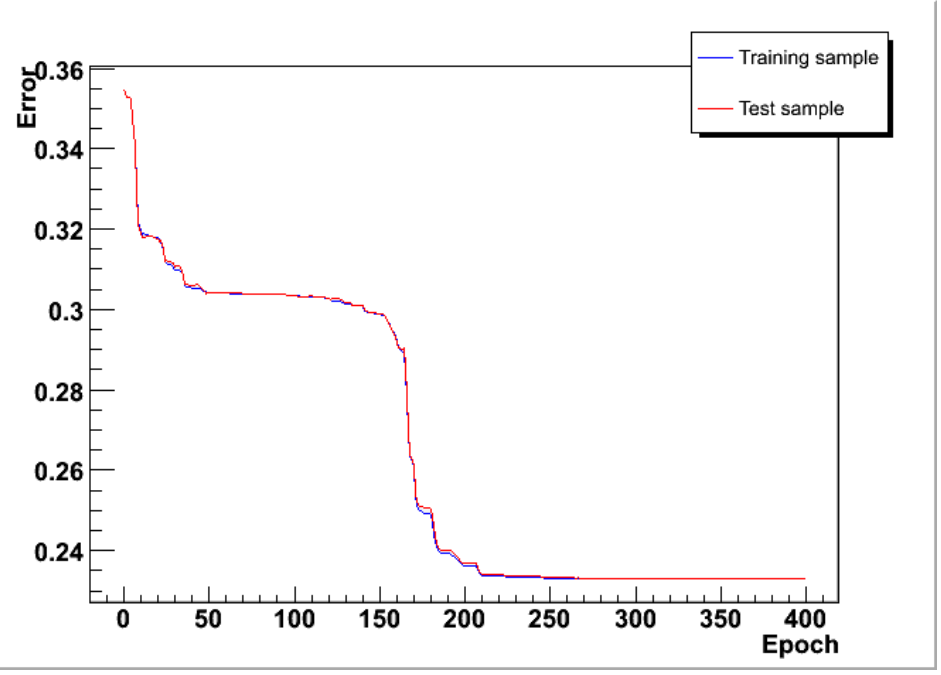

Figure 4.6: Training result from the NN. The blue and red lines show the NN error for the training and test samples, respectively. The NN training algorithm chooses weights which minimize the error for the training sample. After about 250 training epochs, the $\mathrm{NN}$ is unable to find any further improvement. The error for the test distribution is expected to closely follow the training distribution's error unless the NN begins to over-train. If the two lines diverged, we would have to limit the number of epochs to a value less than the value at which the convergence begins. As both lines flatten and show no divergence, we do not need to retrain the $\mathrm{NN}$ with fewer epochs, but the epochs after 250 did not improve the NN performance. 


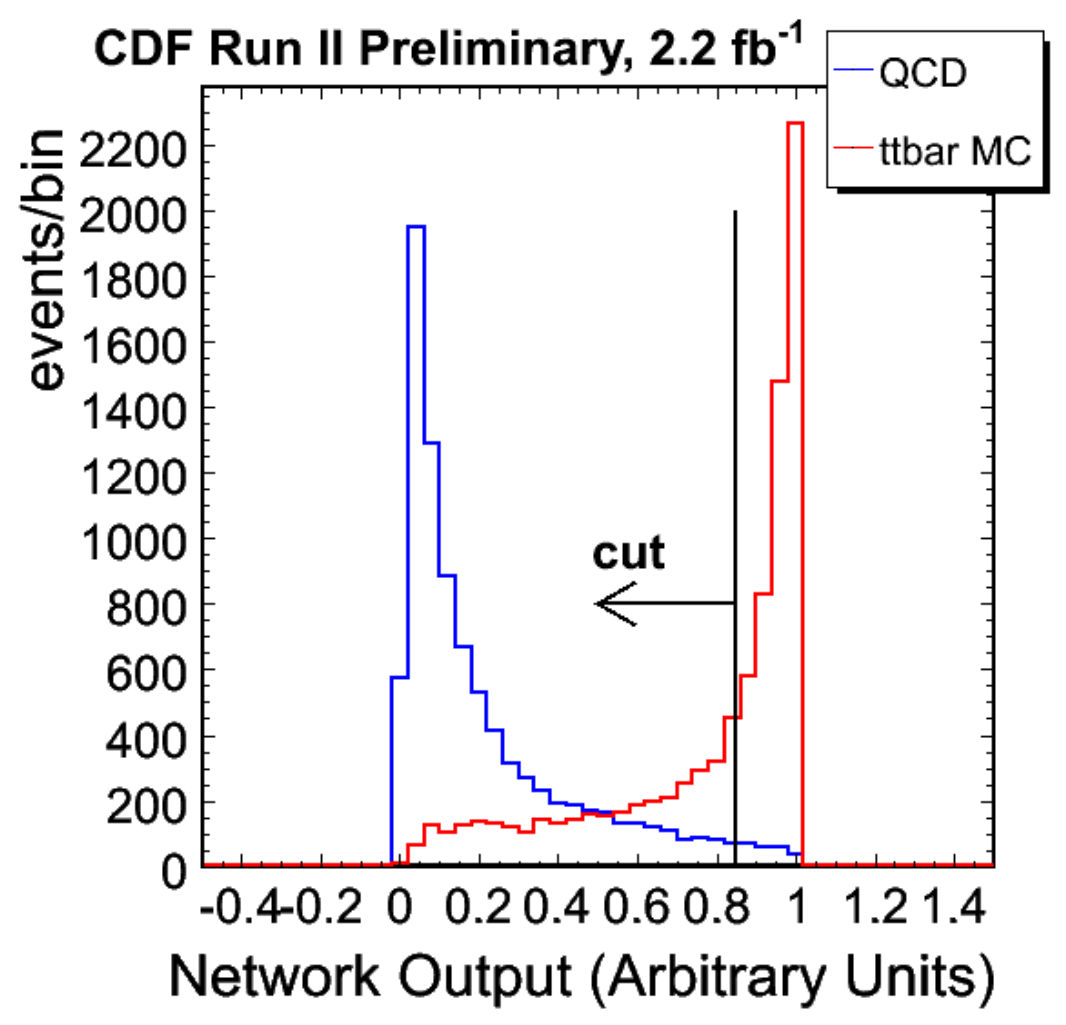

Figure 4.7: Output of the NN after training for the $t \bar{t}$ (red) and QCD multijet (blue) events. We find that the NN provides good separation between the two samples. To reduce the contribution from QCD multijet events in the $\tau+$ jets event selection, we chose to require events to have a $\mathrm{NN}$ output greater than 0.85 to be included in the final selection. This removes approximately $85 \%$ of the QCD multijet events. 


\subsubsection{Neural Network Optimization}

To optimize the NN QCD multijet rejection, we perform the background estimation described later in Sec. 4.3 for several different NN selection values. We assume the $t \bar{t}$ pair production cross section to be $7.4 \mathrm{pb}$ and the top quark mass to be 172.5 $\mathrm{GeV}$. For the NN output selection value, we consider values from 0.2 to 0.95 . Higher values will reduce the contribution from the QCD multijet process, but also reduce the contribution from the $t \bar{t} \rightarrow \tau+$ jets signal. It is important to find the optimum value which balances these two effects. After predicting the signal and background contribution for each possible selection value, we calculate the signal significance $\frac{\sqrt{S}}{\sqrt{S+B}}$ where $S$ and $B$ are the total number of signal and background events as predicted by the background estimate. We chose the value which maximizes this signal significance. As can be seen in Fig. 4.8, we find 0.85 to be the most optimal value for QCD multijet rejection. Of the initial 162 selected events, 41 pass the NN requirement with a selection value of 0.85 .

As a crosscheck, we also looked at $t \bar{t}$ acceptance and sample purity to optimize the NN selection value. The sample purity is defined as the number of expected $t \bar{t} \rightarrow \tau+$ jets events divided by the total number of observed events. The distribution from both of these variables as a function of the selection value and as a function of each other are shown in Fig. 4.9. The chosen selection value of 0.85 corresponds with where the $t \bar{t}$ acceptance begins to significantly decline.

\subsection{Background Estimate}

Due to difficulty in modeling the $b$ quark tagging algorithms and the production of heavy flavor quarks in association with $W$ bosons with $\mathrm{MC}$, we use a data-driven approach to estimate the background contribution similar to that described in [72]. First, we estimate the contribution from each process before the b-tagging requirement is applied (pretag). We calculate the contributions from electroweak background processes which have a minimal contribution to the final total (diboson, single top quark production, and $Z+$ jets events), as well as the signal contribution, by using the theoretical cross 


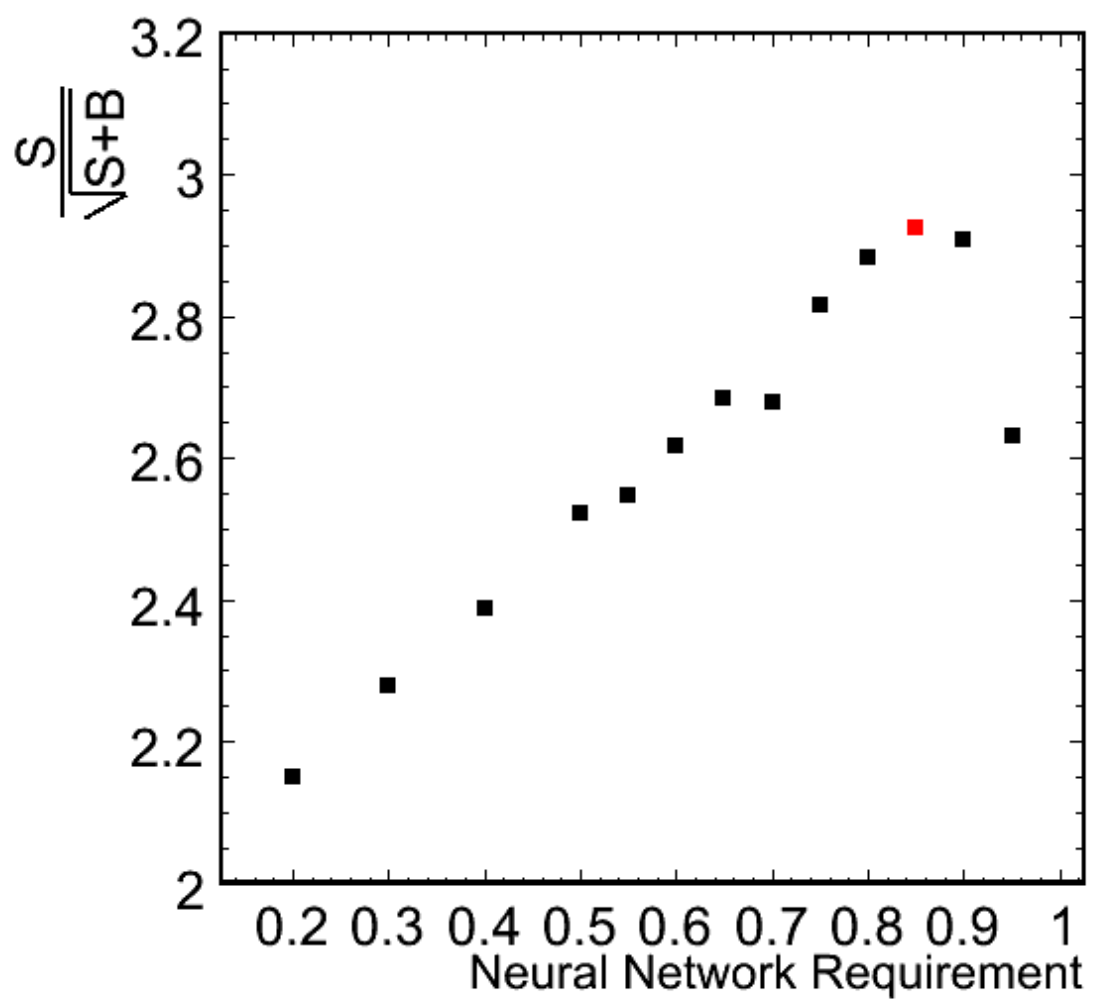

Figure 4.8: $\frac{S}{\sqrt{S+B}}$ versus NN selection value. The red point shows the optimized value at 0.85 . 

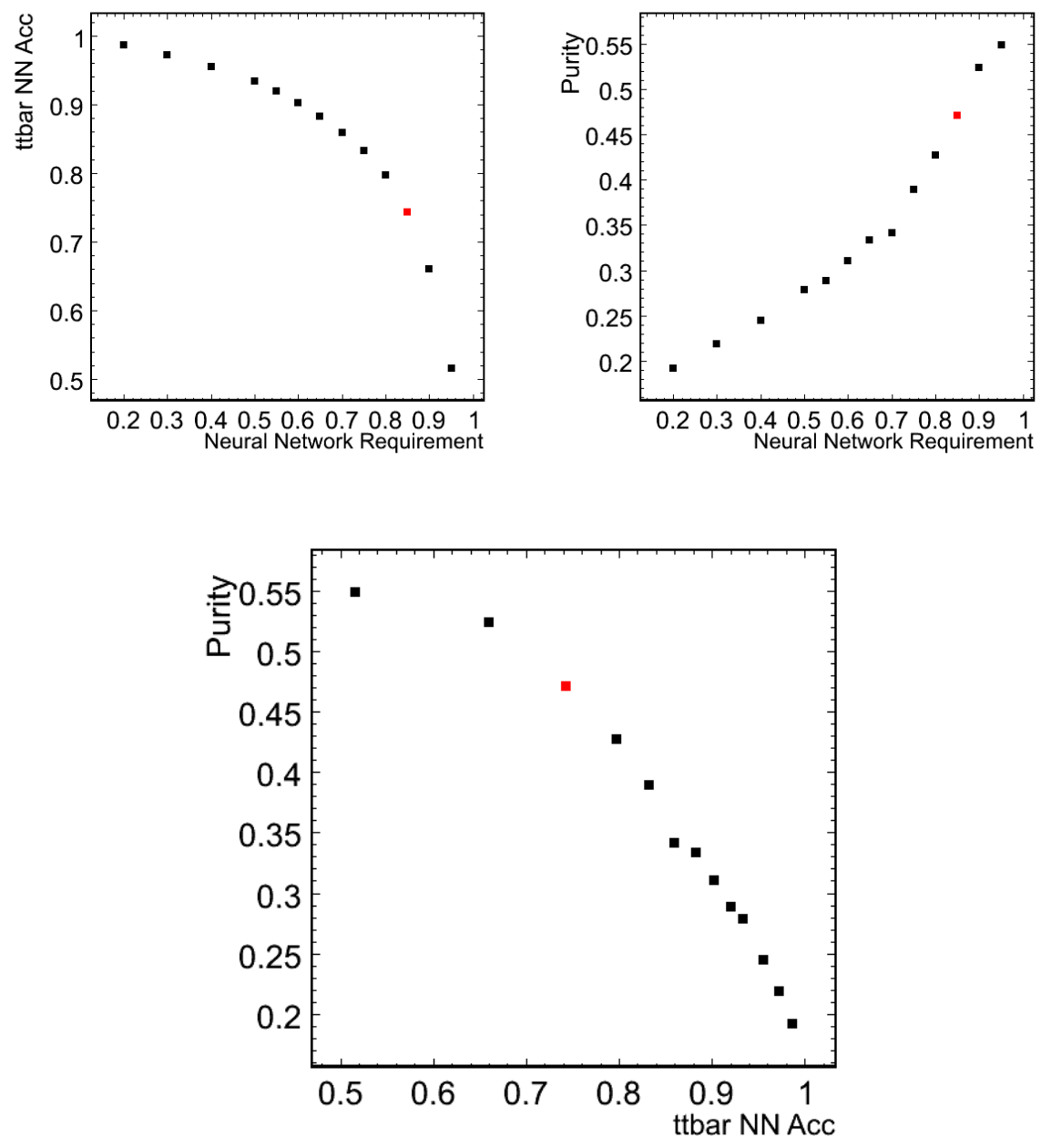

Figure 4.9: $t \bar{t}$ acceptance (top left) and sample purity (top right) versus NN selection value. In the bottom row is the purity vs $t \bar{t}$ acceptance. In all three distributions the chosen NN selection value of 0.85 is highlighted in red. 
section for each process along with the acceptance from MC simulation scaled by the appropriate data/MC scale factors (defined as the ratio of the efficiency of a process in data and $\mathrm{MC}$, which is used to scale the weight of the $\mathrm{MC}$ events so they properly model the data) and the total integrated luminosity of the data sample. The acceptance for any of these processes before the b-tagging requirement is defined as:

$$
A c c=A c c_{\text {evtSel }}^{M C} \cdot \frac{\epsilon_{\text {trigger }}^{\text {data }}}{\epsilon_{\text {trigger }}^{M C}} \cdot \frac{\epsilon_{z 0}^{\text {data }}}{\epsilon_{z 0}^{M C}} \cdot \frac{\epsilon_{\tau I D}^{\text {data }}}{\epsilon_{\tau I D}^{M C}},
$$

where $A c c_{\text {evtSel }}^{M C}$ is the acceptance for events to pass the event selection (defined as the percentage of the total number of generated events surviving the selection requirements) as measured on $\mathrm{MC}$ events before the b-tagging selection requirement, $\epsilon_{\text {trigger }}$ is the efficiency of the trigger selection, $\epsilon_{z 0}$ is the efficiency for the primary vertex of the event to be within $60 \mathrm{~cm}$ of the center of the detector (required to ensure the daughter particles of the process can be well measured by the CDF detector), and $\epsilon_{\tau I D}$ is the efficiency for $\tau$ 's to pass the $\tau$ selection requirements detailed in Tab. 3.1 (where ID standard for identification). $\mathrm{MC}$ and data designations refer to the quantity being measured in MC events or data events, respectively. The three factors of $\frac{\epsilon^{d a t a}}{\epsilon^{M C}}$ are referred to as data/MC scale factors $(\mathrm{SF})$ and are used to rescale measurements made in $\mathrm{MC}$ for factors in which the efficiency is known to be different for data and MC events. We can thus rewrite Eqn. 4.2 as:

$$
A c c=A c c_{\text {evtSel }}^{M C} \cdot S F_{\text {trigger }} \cdot S F_{z 0} \cdot S F_{\tau I D}
$$

It should be noted that the trigger and primary vertex efficiencies in MC are already taken into account in the event selection acceptance as we only select MC events that pass the trigger selection and have a primary vertex within $60 \mathrm{~cm}$ of the center of the detector. The scale factor for the trigger is the product of the level 1 and level 2 trigger scale factors discussed in Sec. 3.1. As the $\epsilon_{z 0}^{M C}$ is equal to 1 due to the explicit selection of $\mathrm{MC}$ events passing this requirement, $S F_{z 0}$ is equal to the $\epsilon_{z 0}^{\text {data }}$. The efficiency $\epsilon_{z 0}^{\text {data }}$ is measured by fitting the luminosity weighted profile of the primary vertex $z_{0}$ position in min-bias data. The efficiency is calculated as the integral of this fit from a $z_{0}$ of $-60 \mathrm{~cm}$ to $+60 \mathrm{~cm}$ divided by the integral of the same function from $-\infty$ to $+\infty$ [73], 


\begin{tabular}{|c|c|c|}
\hline Process & Cross Section (pb) & Acceptance (\%) \\
\hline$t \bar{t}\left(M_{\text {top }}=172.5 \mathrm{GeV}\right)$ & $7.4 \pm 1.0$ & 0.1885 \\
\hline Single Top - s channel & $0.88 \pm 0.05$ & 0.0157 \\
Single Top - t channel & $1.98 \pm 0.08$ & 0.0101 \\
WW & $12.4 \pm 0.25$ & 0.0031 \\
WZ & $3.96 \pm 0.06$ & 0.0039 \\
ZZ & $1.58 \pm 0.05$ & 0.0019 \\
$Z+$ jets & $787.4 \pm 50$ & 0.0002 \\
\hline
\end{tabular}

Table 4.1: Theoretical cross sections [3] and measured MC acceptances used to calculate the signal and background contributions in the selected data events. The acceptances given here do not include the b-tagging selection requirements.

and it is measured to be $97.20 \% \pm 0.05 \%$ [73] [74]. Finally, $S F_{\tau I D}$ is measured from the efficiency of $\tau$ identification in the data and MC. The $\tau$ identification efficiency is calculated as the number of $\tau$ candidates which pass the $\tau$ identification requirements listed in Tab. 3.1 divided by the number of $\tau$ candidates which pass the selection except with the visible mass and isolation requirements removed. This efficiency is calculated by selecting $W \rightarrow \tau \nu$ events in $\mathrm{MC}$ data. Two different models are used to predict the contribution from QCD multijet events in the data. In one model, the estimate is made by fitting the difference in $\phi$ between the $\tau$ candidate and the hadronic recoil. The other model fits a distribution of the component of the hadronic recoil vector parallel to the direction of travel of the $\tau$ candidate. The $\tau$ identification scale factor is measured individually based on each model and the results are averaged to give a $S F_{\tau I D}$ of $0.95 \pm 0.02[75$. The uncertainty is the sum in quadrature of the statistical uncertainty of the measurement and the systematic uncertainty taken as the difference between the results from the two QCD multijet models.

For any process which we simulate with MC, the Acc as calculated in Eqn. 4.3 is be multiplied by the total integrated luminosity of the data and the cross section for the given process to calculate its contribution to the data sample. The cross sections and acceptances for these processes are listed in Tab. 4.1 and a summary of the scale factors is given in Tab. 4.2. With these contributions known, we are only left to evaluate contributions for QCD multijets and $W+$ jets events. 


\begin{tabular}{|c|c|}
\hline Scale Factor & Value \\
\hline Trigger & Event dependent, overall $<0.963 \pm 0.008$ (see Sec. 3.1) \\
$z_{0}$ & $0.9720 \pm 0.0005$ \\
$\tau$ ID & $0.95 \pm 0.02$ \\
\hline
\end{tabular}

Table 4.2: Data/MC scale factors used to calculate the signal and background contributions in the selected data events.

To do this, we use the shape of the NN output distribution for each contribution, as well as the data, before any $\mathrm{NN}$ selection requirement is applied. We then fit these distributions to the data using a binned Poisson likelihood function which allows the contribution from $W+$ jets and QCD multijets events to float while constraining the contributions from all other processes to their previously calculated values. From this fit, whose output shown in Fig. 4.10, we evaluate the percentage of the data events above a certain NN output value which are coming from QCD multijets. Since the peak of the QCD multijets distribution occurs around a $\mathrm{NN}$ value of 0.1 , for any $\mathrm{NN}$ output value above 0.4 the QCD multijets fit is generally derived from data outside of the signal region, and the contribution in the signal region is extrapolated from the fit. Finally, the $W+$ jets category is used as a "catch-all" for any remaining data events:

$$
N_{W+\text { jets }}^{\text {pretag }}=N_{\text {data }}^{\text {pretag }}-N_{E W K+\text { top }}^{\text {pretag }}-f_{Q C D} \cdot N_{\text {data }}^{\text {pretag }},
$$

where $\mathrm{N}_{\text {data }}^{\text {pretag }}$ is the total number of events in the data before the b-tagging requirement, $\mathrm{f}_{Q C D}$ is the fraction of QCD multijets events which survive the NN selection requirement, and $\mathrm{N}_{E W K+\text { top }}$ is the total number of events from diboson, single top, $Z$ + jets, and $t \bar{t}$ production. The fraction $\mathrm{f}_{Q C D}$ is simply defined as the number of $\mathrm{QCD}$ multijet events from the fit which have a $\mathrm{NN}$ value above the selection requirement divided by the total number of data events which survive the NN selection. We find the pretag $\mathrm{f}_{Q C D}$, shown in Fig. 4.10 , is $73 \%$.

At this point, we have completely estimated the contribution from all processes in the data before the b-tagging requirement is applied. Next, we use this estimate as the starting point for evaluating the contribution from each process after the b-tagging 


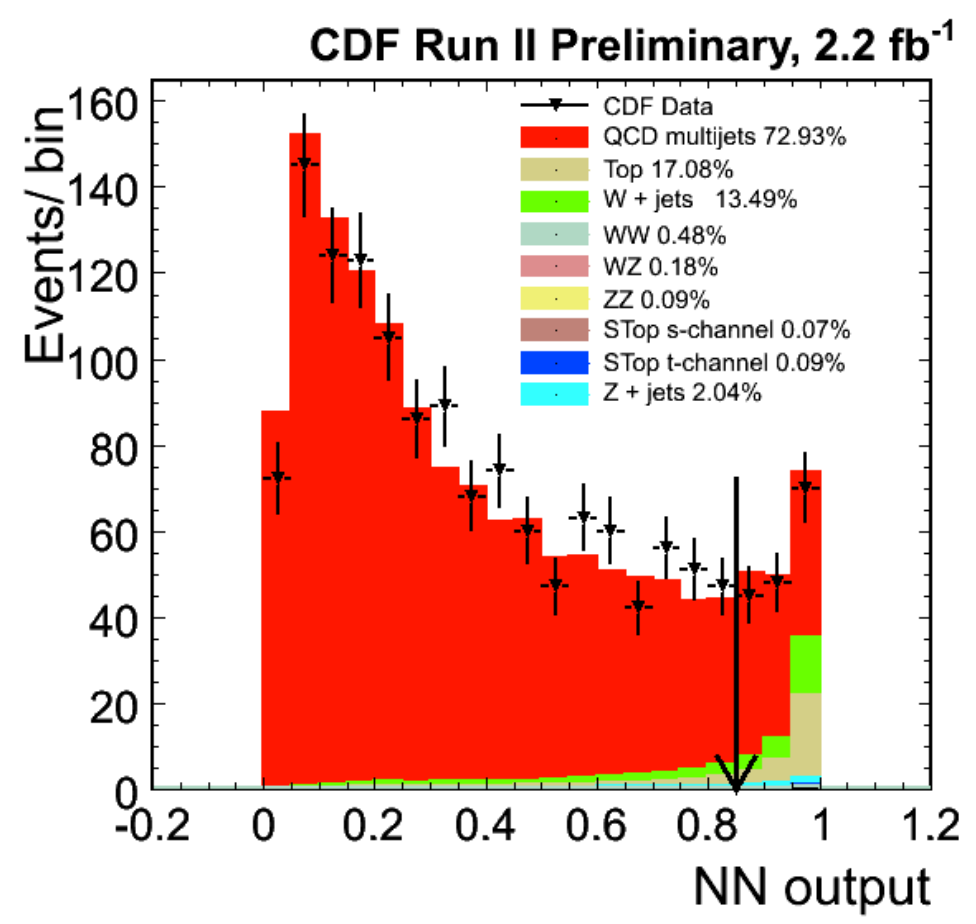

Figure 4.10: NN output distributions for all processes fit to data before the b-tagging requirement is applied to the event selection. The red distribution shows the QCD multijet distribution which dominates the data before the NN selection is applied. The NN selection value of 0.85 is marked with an arrow. We select events above this threshold. The percentages in the legend give the percentage of events from each source after the NN requirement of 0.85 . 
requirement is applied. Since the heavy flavor tagging efficiency is over-estimated [76] and the mistagging of light flavor quarks is very poorly modeled (underestimated) [77] in the $\mathrm{MC}$, we introduce a b-tagging scale factor [76] to reduce the weight of b-tagged events so the predicted number of b-tagged MC events matches the data and a parameterized mistag probability known as the mistag matrix [77] to better model mistagging of light quark jets. The b-tagging scale factor is the ratio of the b-tagging efficiency in data and MC. This efficiency is measured in [76] by selecting dijet events with two jets opposite in $\phi$. One jet is b-tagged and the other jet contains a $9 \mathrm{GeV}$ electron (the electron jet) indicative of a semi-leptonic $b$ decay. The efficiency is simply defined as the fraction of these events in which the electron jet is b-tagged. The full uncertainty on the SF is described in [76], but the lead uncertainty comes from the background contribution from mistagged jets and electrons from $\gamma$ conversion. The mistag matrix is parameterized in terms of the jet $E_{T}, \eta, \phi$, the total sum $E_{T}$ of all jets in the event, and the total number of tracks identified in the silicon vertex detector. The exact value of the mistag probability depends on the parameters of the jet. The mistag rate returns the tag rate as measured from data for a jet with the input parameters and tends to be on the order of 2-7\% [77]. Systematic uncertainties on this tag rate are evaluated for the sample composition, jet $\sum E_{T}$ distribution, and bias from triggered jets as described in [78].

For each MC event, we assign a tagging probability to each jet. If the jet is matched to a heavy flavor hadron ( $\mathrm{B}$ or $\mathrm{C}$ hadron), it is assigned a tagging probability, $p_{\text {tagged }}$, equal to the b-tagging scale factor of $0.95 \pm 0.05$ [76] if it is b-tagged or 0 if it is not b-tagged. If the jet is not matched to a heavy flavor hadron, it is assigned a tagging probability from the mistag matrix regardless of if it is b-tagged or not. With each jet assigned a b-tagging probability, $p_{\text {tagged }}$, we can calculate the probability $\left(P_{\text {tagged }}^{\text {evt }}\right)$ of the event having at least 1 jet tagged as coming from a $b$ quark:

$$
P_{\text {tagged }}^{\text {evt }}=\left(1-\prod_{i}^{N j e t s}\left(1-p_{\text {tagged }}^{i}\right)\right) .
$$

This event b-tagging probability is then used to calculate the tagging efficiency for 
any MC process XX:

$$
\epsilon_{X X}^{t a g}=\frac{\sum_{j}^{N_{X X}^{\text {pretag }}} P_{\text {tagged }}^{j}}{N_{X X}^{\text {pretag }}} .
$$

The exact value of $\epsilon^{t a g}$ is dependent on the number of jets from heavy flavor quarks and the mistag rate of jets from light flavor. However, assuming the small mistag rate to be negligible and the b-tagging efficiency to be $40 \%$ as measured for $b$ quarks from top decays discussed in Sec. 3.2.2 $\epsilon^{\text {tag }}$ would be roughly $62 \%, 38 \%$, and $0 \%$ for events with 2,1 , and 0 jets from $b$ quarks, respectively.

For each MC modeled process (including the $t \bar{t}$ signal), we estimate the number of events passing the b-tagged selection by multiplying this b-tagging efficiency by the number of estimated events before the b-tagging requirement. The resulting contributions to the b-tagged sample from $t \bar{t}$, single top, $W W, W Z, Z Z$, and $Z+$ jets events are all listed in the appropriate rows of Tab. 4.4. The calculation of the contribution to the b-tagged sample is slightly more involved for $W+$ jets events since we break down the contribution into $W+$ heavy flavor and $W+$ light flavor. Heavy flavor events contain true $b$ or $c$ quark while light flavor events have no real heavy flavor and instead have a mistagged jet from a light flavor quark. First, we calculate the contribution from $W+$ heavy flavor:

$$
\begin{aligned}
N_{W+h f}^{\text {tag }} & =\left(N_{\text {data }}^{\text {pretag }}-N_{\text {top }}^{\text {pretag }}-N_{E W K}^{\text {pretag }}-N_{Q C D}^{\text {pretag }}\right) \cdot f_{H F} \cdot K \cdot \epsilon_{W+h f}^{\text {tag }} \\
& =N_{W+j e t s}^{\text {pretag }} \cdot f_{H F} \cdot K \cdot \epsilon_{W+h f}^{\text {tag }}
\end{aligned}
$$

where $f_{H F}$ is the fraction of events with at least one jet matched to heavy flavor, $\mathrm{K}$ is a data/MC SF which is used to account for an underestimation in the amount of heavy flavor in the $W+$ jets MC [79], and $\epsilon_{W+h f}^{t a g}$ is the tagging efficiency for $W+$ heavy flavor events. The contribution $N_{W+h f}^{t a g}$ as well as $f_{H F}$ and $\epsilon_{W+h f}^{\operatorname{tag}}$ are each calculated separately for $W+b \bar{b}, c \bar{c}$, and $c$ processes. For each of the three processes, $f_{H F}$ is calculated by dividing the total number of events with the correct number of heavy flavor quarks by the total number of events seen across ALPGEN+PythiA MC generated with both heavy flavor and light flavor matrix elements. The efficiency $\epsilon^{t a g}$ is measured similarly for all three heavy flavor processes. The values for $f_{H F}$ and $\epsilon^{t a g}$ for $W+$ jets $\mathrm{MC}$ events with $1 b, 2 b, 1 c$, and $2 c$ quarks are listed in Tab. 4.3 . Since 


\begin{tabular}{|c|c|c|}
\hline $\begin{array}{c}\text { Number of heavy } \\
\text { flavor quarks }\end{array}$ & $f_{H F}$ & $\epsilon^{\text {tag }}$ \\
\hline 1b & 5.17 & 27.90 \\
2b & 4.77 & 42.04 \\
1c & 17.80 & 10.08 \\
2c & 9.15 & 16.37 \\
\hline
\end{tabular}

Table 4.3: Heavy flavor fractions $f_{H F}$ and tagging efficiencies $\epsilon^{t a g}$ for $W+$ jets events with $1 b, 2 b, 1 c$, and $2 c$ quarks as calculated from Alpgen+Pythia MC.

the $W+$ jets $\mathrm{MC}$ is found to have a lower contribution from heavy flavor than is seen in the data, a heavy flavor fraction data/MC scale factor $\mathrm{K}$ is introduced. This scale factor can viewed as the ratio of the fraction of data $W+$ jets events with a heavy flavor quark to the fraction of MC $W+$ jets events with a heavy flavor quark. It is measured to be $1.5 \pm 0.3[\underline{80}$ ] by comparing heavy flavor fractions in data and $\mathrm{MC} W$ + jets events with the help of a NN which separates jets from heavy and light flavor quarks in the 1 and 2 jet bins. The resulting contribution from $W+$ heavy flavor events in the b-tagged sample calculated with Eqn. 4.8 are listed in the appropriate rows of Tab. 4.4 .

With the $W+$ heavy flavor estimation in hand, we can use the mistag matrix to determine how many of the remaining pretag $\mathrm{W}+$ jets events, i.e. those not associated with heavy flavor, will contribute to the b-tagged sample. We calculate the contribution from mistagged $W+$ light flavor $(W+$ lf $)$ as:

$$
N_{W+l f}^{\text {tag }}=\left(N_{\text {data }}^{\text {pretag }}-N_{Q C D}^{\text {pretag }}-N_{\text {top }}^{\text {pretag }}-N_{E W K}^{\text {pretag }}-N_{W+h f}^{\text {pretag }}\right) \cdot \epsilon_{\text {mistag }}^{\text {tag }}
$$

where $N_{W+h f}^{\text {pretag }}$ contribution is equal to the contribution of b-tagged heavy flavor calculated in Eqn. 4.8 but without the $\epsilon_{W+h f}^{t a g}$ term, and $\epsilon_{\text {mistag }}^{\text {tag }}$ is equivalent to the probability of an event having at least 1 b-tag as calculated in Eqn. 4.5 except now each jet's b-tagging probability comes from the result of the mistag matrix. The resulting $W+$ lf contribution is also listed in Tab. 4.4

Finally, with all contributions except the QCD multijets accounted for after the b-tagging requirement, we again use a binned Poisson likelihood fit to estimate the contribution from QCD multijets. This time, we fit the NN output distribution of 
data selected with the b-tag requirement to two templates. One template is built from events passing the QCD model selection described in Sec. 4.2 including the requirement of at least 1 b-tagged jet. The second template is built by summing together the contributions from all other processes as listed in Tab. 4.4. The amplitude of both templates is allowed to float in the fit, however, the fractional contribution from each process in the non-QCD template is kept constant. Just as in the pretag scenario, the QCD multijets NN output distribution peaks near 0, so the main contribution to the fit comes from events outside of the signal region, and the expectation in the signal region is then extrapolated. The result of the fit can be seen in Fig. 4.11. The total contribution from b-tagged QCD multijets events is then calculated as the integral of the fit QCD multijets template above the selected NN value. The fraction of tagged QCD multijet events $f_{Q C D}^{t a g}$ is found to be $44.5 \%$ and the total number of estimated QCD multijet events in the data selection is 18.24 events.

From the background estimate, with a $t \bar{t}$ pair production cross section of $7.4 \mathrm{pb}^{-1}$ and a top quark mass of $172.5 \mathrm{GeV}$, we expect to observe 39 events with approximately $18 t \bar{t} \rightarrow \tau+$ jets events and 18 QCD multijet events, and we observe 41 events in the data sample (See Tab. 4.4).

\subsection{Composition Validation}

We compare the predicted distributions of several event variables to the distributions observed in the data to validate the background composition prediction. We look at the distributions for:

- Lead jet $E_{T}$

- Second jet $E_{T}$

- Third jet $E_{T}$

- Fourth jet $E_{T}$

- $\tau P_{T}$ 


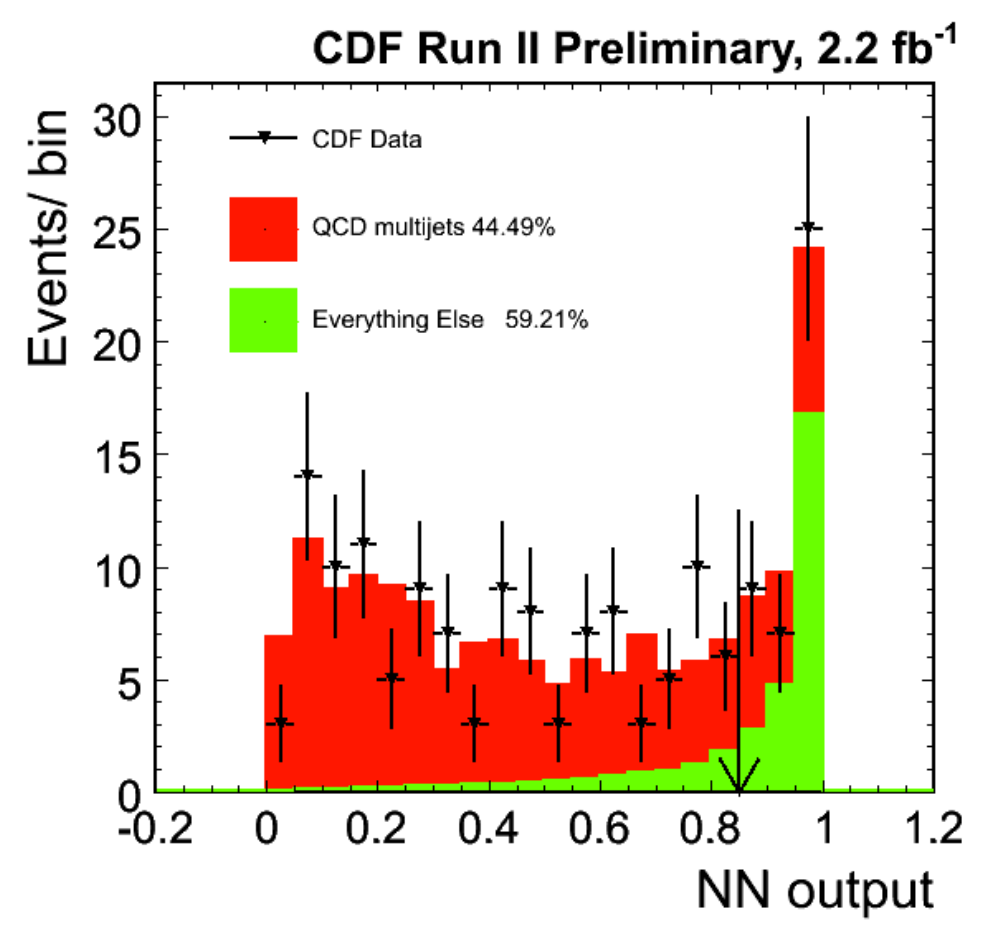

Figure 4.11: NN output distributions for all processes fit to data selected with the btagging requirement. The red distribution is for b-tagged QCD multijet events, and the green distribution is the template formed by combining the expected contributions from all other processes. The NN selection value of 0.85 is marked with an arrow. We select events above this threshold. The percentages given in the legend are the percentage of the events from each contribution after the NN requirement. . 


\begin{tabular}{|l|c|}
\hline Source & Number of Events \\
\hline$W W$ & $0.11 \pm 0.01$ \\
$W Z$ & $0.04 \pm 0.00$ \\
$Z Z$ & $0.04 \pm 0.00$ \\
Stop S-channel & $0.06 \pm 0.01$ \\
Stop T-channel & $0.10 \pm 0.01$ \\
$Z b b$ & $0.29 \pm 0.04$ \\
$W b b$ & $0.57 \pm 0.47$ \\
$W c c$ & $0.34 \pm 0.28$ \\
$W c$ & $0.15 \pm 0.13$ \\
$W+$ lf & $0.46 \pm 0.60$ \\
QCD multijets & $18.24 \pm 4.10$ \\
\hline Total Bkgd & $20.40 \pm 4.18$ \\
\hline Top & $18.17 \pm 2.79$ \\
\hline Total Predicted & $38.57 \pm 5.05$ \\
\hline Observed & 41 \\
\hline
\end{tabular}

Table 4.4: Predicted number of selected $\tau+$ jet events from each considered process after a NN selection value of 0.85 assuming a $t \bar{t}$ pair production cross section of $7.4 \mathrm{pb}$. The estimate predicts roughly 39 events compared to the observed 41 with nearly half of the events coming from $t \bar{t} \rightarrow \tau+$ jets and half from QCD multijet production.

- $E_{\mathrm{T}}$

- b-Tagged jet $E_{T}$

- $\sum E_{T}$ of the $\tau$ and all four jets

- Number of signal tracks in the $\tau$

- Number of b-tagged jets in the event

While some discrepancies can be expected due to the low statistics of only 41 data events, we do not find any concerning discrepancies. A selection of these validation distributions can be seen in Figs. 4.12 through 4.14 

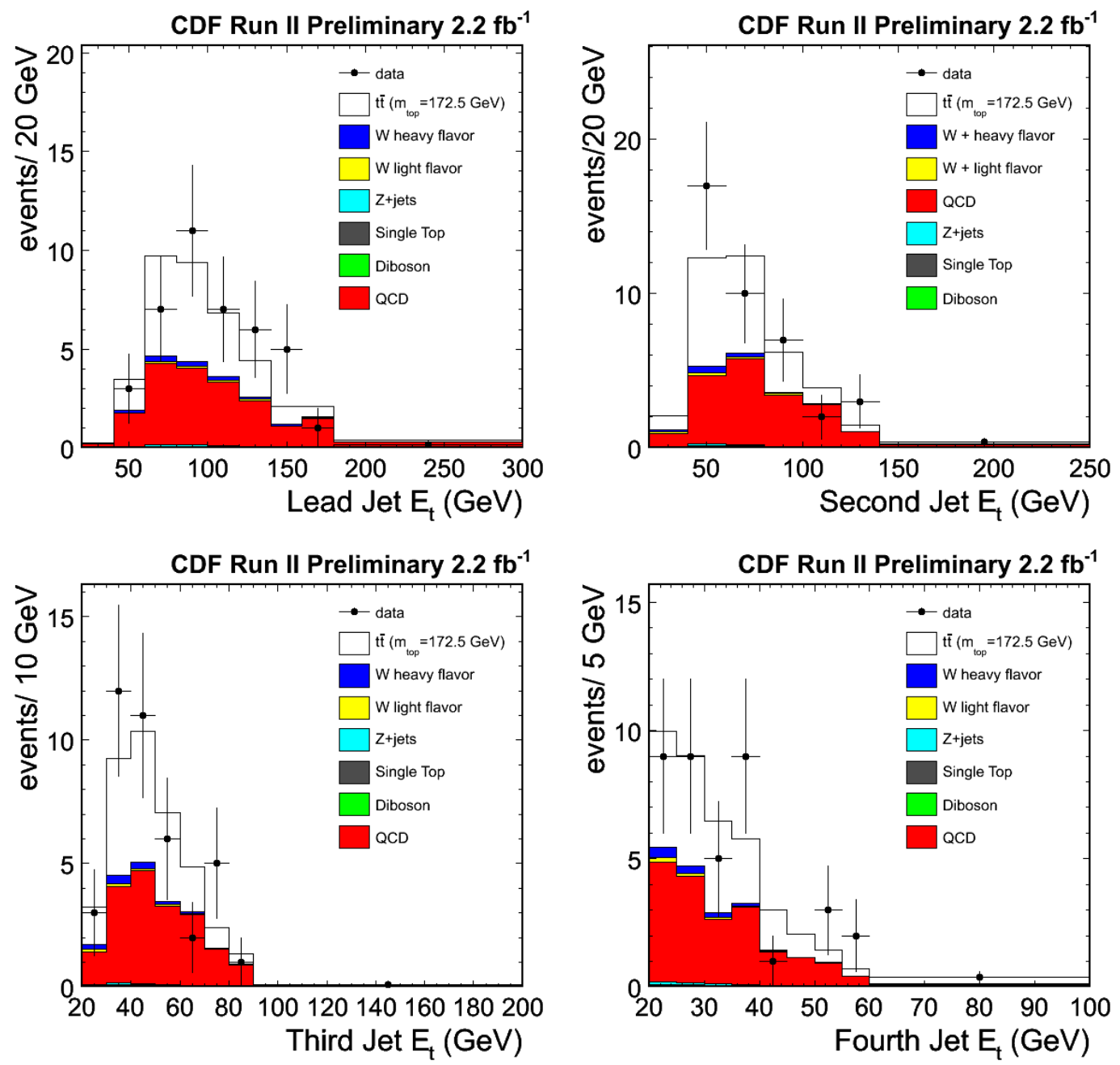

Figure 4.12: The distribution of $P_{T}$ for each jet ranking in order of $P_{t}$ for $\tau+$ jets events. 

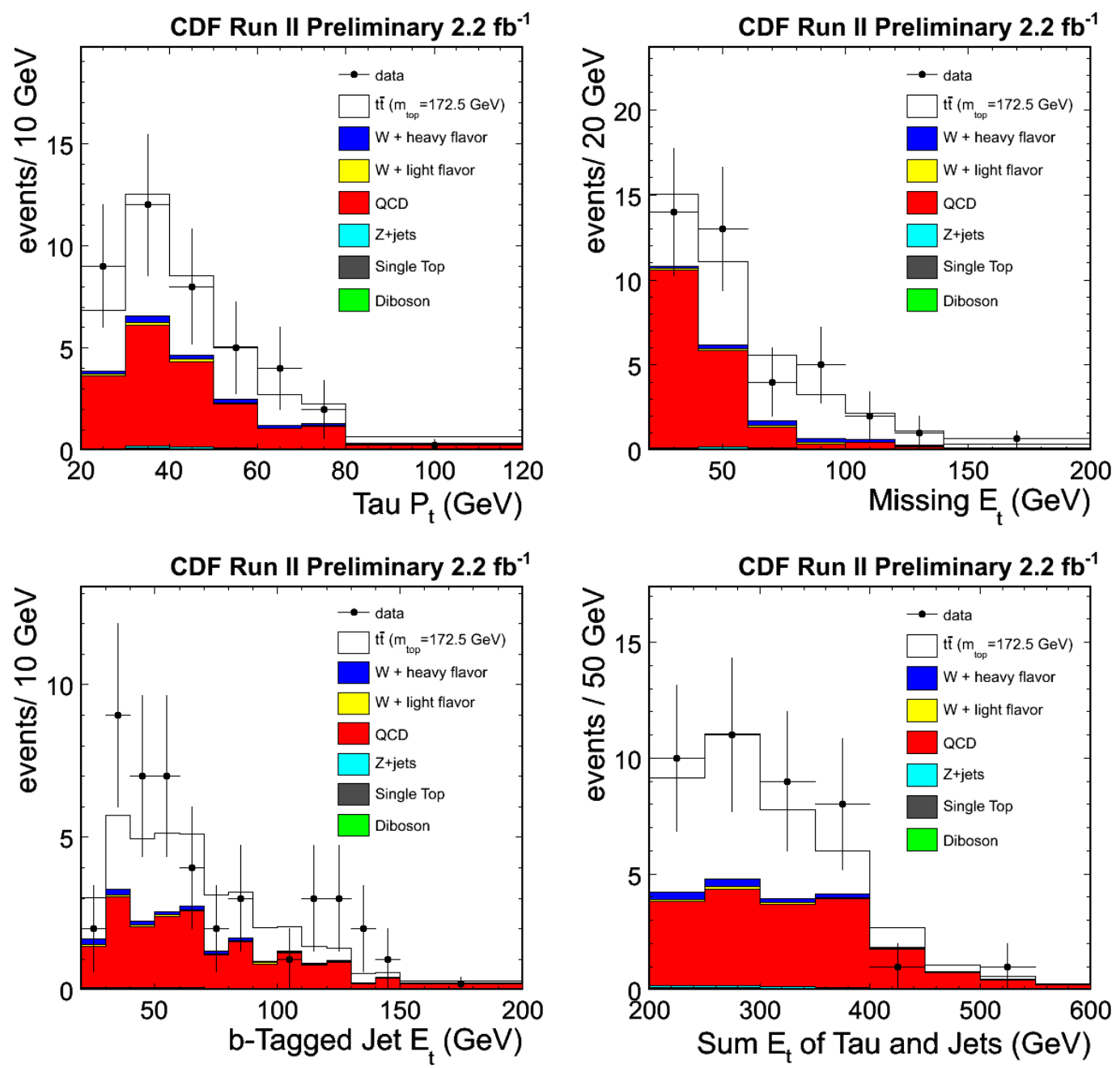

Figure 4.13: The $P_{T}$ of the $\tau$ (upper left), the $E_{T}$ (upper right), the b-tagged jet $E_{T}$ (lower left), and the sum $E_{T}$ of all four jets and the $\tau$ (lower right) are shown. 

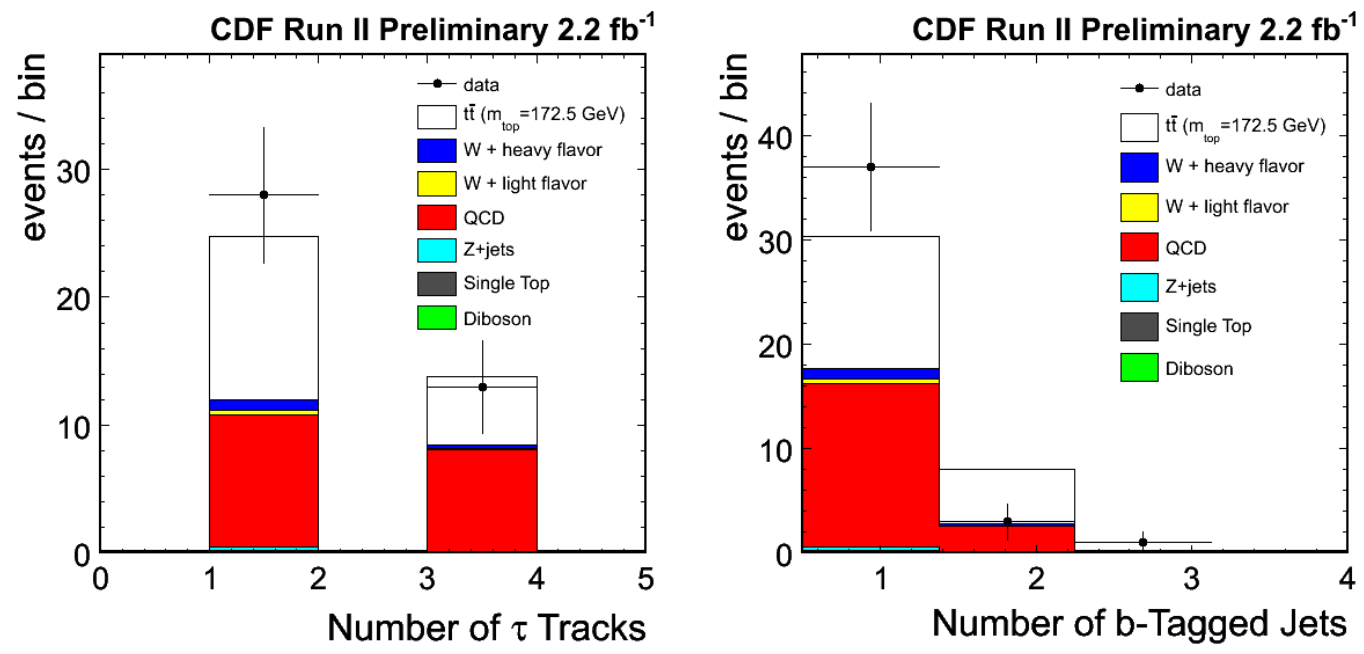

Figure 4.14: Number of tracks in $\tau$ signal cone (left) and number of b-tagged jets (right) for $\tau+$ jets events. 


\section{Chapter 5}

\section{Top Cross Section Analysis}

This chapter describes the $t \bar{t}$ pair production cross section $\left(\sigma_{t \bar{t}}\right)$ measurement in the hadronic $\tau+$ jets decay channel. As of August 2009, $\sigma_{t \bar{t}}$ combined over all other measurements with up to $4.6 \mathrm{fb}^{-1}$ of data at the CDF detector at Fermilab is measured to be $7.5 \pm 0.5 \mathrm{pb}$ assuming a top quark mass of $172.5 \mathrm{GeV}$ [18]. By comparing the result to this value, we test lepton universality in the top quark decay. Additionally, the ability to measure the $t \bar{t}$ cross section in $\tau+$ jets events is a demonstration that we can make measurements in the $t \bar{t} \rightarrow \tau+$ jets decay channel using the CDF detector, and apply the methods described here to the more challenging top quark mass measurement detailed in Chap. 6. This chapter includes a description of the method used in the $\sigma_{t \bar{t}}$ measurement, the important quantities in the measurement, and a full description of the systematic uncertainties on the measurement. The $\sigma_{t \bar{t}}$ measurement itself is largely dependent on the predicted number of background events which has been described previously in Chap. 4 .

\subsection{Cross Section Measurement Method}

In general, a processes cross section is measured as:

$$
\sigma=\frac{N_{d a t a}-N_{b k g d}}{A c c \cdot \epsilon \cdot \mathcal{L}}
$$

where $N_{d a t a}$ and $N_{b k g d}$ are the number of events observed in the data and the number of predicted background events, respectively. The kinematic acceptance for the process being observed (for the case of $\sigma_{t \bar{t}}$, we measure here the acceptance for $p \bar{p} \rightarrow t \bar{t} \rightarrow \tau+$ jets) is $A c c, \epsilon$ is the product of all geometrical and kinematic event selection efficiencies corrected for by data/MC scale factors (SF) when relevant. The SF's used here include 
the trigger, lepton identification (ID), and b-tagging (as defined in Sec. 4.3). Finally $\mathcal{L}$ is the total integrated luminosity of the data.

Recall, the background prediction described in Sec. 4.3 depends on an input $\sigma_{t \bar{t}}$. Therefore, we can not simply use Eqn. 5.1 to measure $\sigma_{t \bar{t}}$. Instead, we construct a Poisson likelihood function (L) which compares the number of events in the data with the signal and background prediction for a given $\sigma_{t \bar{t}}$. We then minimize twice the negative $\log$ of the likelihood $(-2 \cdot \ln L)$ as a function of $\sigma_{t \bar{t}}$ to find the most probable value of $\sigma_{t \bar{t}}$. The factor of 2 has no effect on the best $\sigma_{t \bar{t}}$. It is used to simplify the measured uncertainty on $\sigma_{t \bar{t}}$. A standard statistical deviation, $1 \sigma$ uncertainty, on the minimized parameter of a negative log likelihood function is the difference between the parameter's value at the function minimum and the parameter's value at which the negative log likelihood function rises above its minimum value by a unit of $\frac{1}{2}$ (Note, the value of the negative log likelihood is in arbitrary units and the function's value at the minimum point can be rescaled to any value by the addition of a constant). A proof of this is given in App. A By multiplying the negative log likelihood function by 2, the statistical uncertainty is easily found by finding where the negative log likelihood function increases above its minimum value by 1 unit.

To build the negative log likelihood function, we start with a Poisson probability distribution comparing the number of observed events in the data $\left(N_{\text {observed }}\right)$ to the number of signal and background events predicted for a given $\sigma_{t \bar{t}} N_{\text {predicted }}\left(\sigma_{t \bar{t}}\right)$ :

$$
P\left(N_{\text {observed }} \mid N_{\text {predicted }}\right)=\frac{e^{-N_{\text {predicted }} N_{\text {predicted }}^{N_{\text {observed }}}}}{N_{\text {observed }} !} .
$$

Taking twice the negative log likelihood of this function gives:

$$
-2 \cdot \ln L=-2 \cdot\left(N_{\text {observed }} \cdot \ln \left(N_{\text {predicted }}\left(\sigma_{t \bar{t}}\right)\right)-\ln \left(N_{\text {observed }} !\right)-\left(N_{\text {predicted }}\left(\sigma_{t \bar{t}}\right)\right)\right)
$$

Combining this with Eqn. 5.1 gives:

$$
-2 \cdot \ln L=-2 \cdot\left(N_{d a t a} \cdot \ln \left(\sigma_{t \bar{t}} \cdot D+N_{b}\left(\sigma_{t \bar{t}}\right)\right)-\ln \left(N_{d a t a} !\right)-\left(\sigma_{t \bar{t}} \cdot D+N_{b}\left(\sigma_{t \bar{t}}\right)\right)\right),
$$

where D is the denominator of Eqn. 5.1 and $N_{b}\left(\sigma_{t \bar{t}}\right)$ is the number of events from the background prediction for a given top pair production cross section $\left(\sigma_{t \bar{t}}\right)$. This is the form of the negative log likelihood function used to measure $\sigma_{t \bar{t}}$. 


\subsection{Acceptance and Efficiency for $t \bar{t} \rightarrow \tau+$ jets}

To measure $\sigma_{t \bar{t}}$, we first measure the acceptance, efficiencies, and data/MC SF's needed to compute the denominator of Eqn. 5.1. These have been discussed in the description of the background estimation procedure in Sec. 4.3 , however, we will highlight their particular application to the $t \bar{t}$ cross section here. To evaluate Acc as given in Eqn. 4.3 we first calculate the MC based event selection acceptance $A c c_{\text {evtSel }}^{M C}$. To do this, we use $t \bar{t}$ MC generated with the Pythia MC generator. This MC sample is made up of $5,274,981$ events generated with $M_{t o p}$ of $172.5 \mathrm{GeV}$. All possible decay modes of the top quark (all-hadronic, semi-leptonic, and di-leptonic) are included with their proper branching fractions. Since the final event selection requires 1 or more b-tagged jets and the NN selection, we break down $A c c_{\text {evtSel }}^{M C}$ into three components: the pretag acceptance, b-tagging efficiency $\left(\epsilon_{t a g}\right)$, and the $\mathrm{NN}$ efficiency $\left(\epsilon_{N N}\right)$.

To evaluate the pretag acceptance. We first require that the primary vertex of the MC $t \bar{t}$ events be within $60 \mathrm{~cm}$ of the center of the CDF detector to ensure that the daughter particles from the $t \bar{t}$ decay are observable by the CDF detector. This requirement reduces the total number of generated events to $5,059,191$. Of these roughly 5.1 million events, we find 13592 passing the selection requirements other than the btagging requirement for a total pretag acceptance of $0.269 \%$. This number already includes the acceptance for $\mathrm{MC}$ events to pass the trigger selection $\left(\epsilon_{\text {trigger }}\right)$, but it does not include the level 1 and level 2 data/MC trigger SF's described in Sec. 3.1 . After weighting all of the selected events by the trigger SF's, we are left with 13048.04 events. This result matches the expectation from the trigger SF as the 0.963 level 2 SF far outweighs the small contribution from the level 1 scale factor (see Sec. 3.1) and the total selected events after the trigger weighting is found to be $96.0 \%$ of the selected events before the weighting.

Next, we apply the b-tagging requirement as is described in the Sec. 4.3 and find $\epsilon_{t a g}$ to be $63.1 \%$. Finally, we evaluate $\epsilon_{N N}$. We find $74.3 \%$ of selected events pass the NN selection of $>0.85$. Combining the pretag acceptance with $\epsilon_{t a g}, \epsilon_{N N}$, and the trigger SF gives us a total selection acceptance of $0.12 \%$. 


\begin{tabular}{|l|c|}
\hline Acc & \\
\hline Pretag Acceptance (including trigger acceptance) & $0.00269 \pm 0.00002$ \\
$\epsilon_{N N}$ & $0.731 \pm 0.014$ \\
$\epsilon_{\text {tag }}$ & $0.631 \pm 0.009$ \\
\hline Scale Factors & \\
\hline Lepton ID & $0.95 \pm 0.02$ \\
Trigger (average 11 and 12 combined) & $0.960 \pm 0.008$ \\
z0 & $0.9720 \pm 0.0005$ \\
\hline
\end{tabular}

Table 5.1: Acceptances, efficiencies, and scale factors for $\sigma_{t \bar{t}}$ measurement. Note, the Pretag acceptance includes $\epsilon_{\text {trigger }}$ but not the trigger SF. The trigger SF varies from event to event, but the value listed here is the average SF over all $t \bar{t} \mathrm{MC}$ events considered in this analysis. These are the values used to calculate the total $t \bar{t}$ acceptance in Eqn. 4.3 .

In summary, all numbers required to calculate $A c c$ as given in Eqn. 4.3 are given in Tab. 5.1

\subsection{Fitting for $\sigma_{t \bar{t}}$}

To extract $\sigma_{t \bar{t}}$, we evaluate the $-2 \cdot \ln L$ function from Eqn. 5.4 over a range of 100 cross sections from 5 to $15 \mathrm{pb}$ with a step size of $0.1 \mathrm{pb}$. For each cross section, we estimate the full signal and background prediction as described in Sec. 4.3. The diboson, single top, and $Z+$ jet estimates have no dependence on $\sigma_{t \bar{t}}$, so their contributions remain the same for all evaluated $\sigma_{t \bar{t}}$. Since the QCD multijet fit is mainly derived from events outside of the signal region (NN output values before 0.85 ), we do not expect the result to be sensitive to changes in $\sigma_{t \bar{t}}$. As a result, we do not reperform the fit at each input value of $\sigma_{t \bar{t}}$. The only changes are the number of predicted $t \bar{t}$ events and the $W+$ jets contributions $(W+b b, W+c c, W+c, W+l f)$. We then fit a $2^{\text {nd }}$ order polynomial around the minimum of the $-2 \cdot \ln L$ function given in Eqn. 5.4 to derive the measured cross section and its statistical uncertainty. To avoid effects from possible non-Gaussian shapes in the tails of the likelihood function, we define a fit window \pm 1 $\mathrm{pb}$ around the value of $\sigma_{t \bar{t}}$ which minimizes the negative log likelihood function. Once the fit is performed within the initial fit window, we expand the fit window to $\pm 1 \sigma$ around the fit central value and refit the $-2 \cdot \ln L$ function. We loop this refitting 3 
times which we find is enough to give a stable fit result. The negative log likelihood function and final fit are shown in Fig. 5.1. We measure the cross section to be:

$$
\sigma_{t \bar{t}}=8.8 \pm 3.3 \text { (stat.) pb. }
$$

We describe the calculation of the systematic uncertainties on this measurement next.

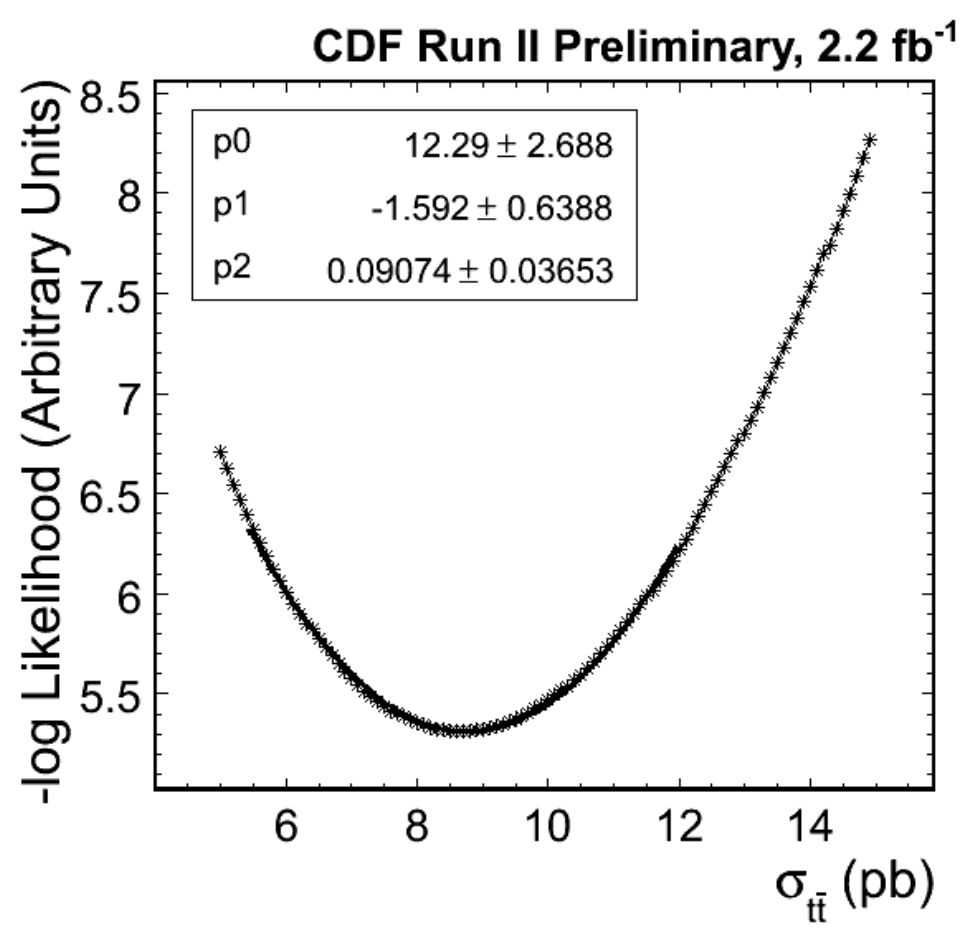

Figure 5.1: The function $-2 \cdot \ln L$ versus input $\sigma_{t \bar{t}}$ as defined in Eqn. 5.4 . The stars $\left(^{*}\right)$ show the calculated values of the function for various input values of $\sigma_{t \bar{t}}$ while the solid line is the fit of a second order polynomial. The cross section is taken to be value which minimizes the fitted function and the statistical uncertainty is the distance along the $\mathrm{x}$-axis traveled from the minimum value before the $-2 \cdot \ln L$ rises to a value of 1 above its minimum.

\subsection{Systematics}

We consider several sources of systematic uncertainties for the cross section measurement. Uncertainties on the acceptance include the jet energy scale (JES), initial and final state radiation, color reconnection, the parton showering model, and the parton distribution functions. Heavy and light flavor tagging SF's, the lepton identification SF, 


\begin{tabular}{|l|c|c|}
\hline Systematic & $\delta \sigma(\mathrm{pb})$ & $\delta \sigma / \sigma(\%)$ \\
\hline Jet Energy Scale & 0.6 & 6.9 \\
IFSR & 0.5 & 5.7 \\
Color Reconnection & 0.4 & 4.6 \\
Tagging & 0.4 & 4.6 \\
Mistag Matrix & 0.1 & 1.1 \\
QCD Fraction & 1.8 & 20.5 \\
K-Factor & 0.1 & 1.1 \\
Parton Showering & 0.5 & 6.0 \\
Lepton ID & 0.2 & 2.3 \\
Trigger Efficiency & 0.1 & 1.1 \\
PDF & 0.5 & 5.7 \\
Luminosity & 0.5 & 6.0 \\
\hline Total & 2.2 & 25.0 \\
\hline
\end{tabular}

Table 5.2: Systematic uncertainties for the $t \bar{t}$ cross section measurement in the $\tau+$ jets decay channel. The uncertainties are given as well as the fractional uncertainty.

and the trigger SF all contribute to the uncertainty on the efficiency. The background uncertainty is made up of the QCD multijet fraction and the $W+$ heavy flavor $\mathrm{K}$-factor uncertainties. Finally, we consider a $6.0 \%$ uncertainty on the luminosity.

To measure the effect of each systematic uncertainty, we vary the parameter of interest by $\pm 1 \sigma$ or substitute a new model for the process in question and reperform the cross section measurement. The statistical uncertainty of the cross section measurement is driven by the data which remains the same for all systematic measurements. We can thus attribute any change in the measurement to the systematic source in question. Even in cases where a new MC sample is used to model a systematic shift, the samples used have large enough statistics such that its statistical uncertainty is negligible. In the following sections, we describe how each source of uncertainty is measured. Table 5.2 lists the contribution from each source. We measure a total systematic uncertainty of $2.2 \mathrm{pb}$ on the top pair cross section.

\subsubsection{Acceptance Uncertainties}

The acceptance term in the cross section measurement is affected by the JES, the amount of initial and final state radiation, color reconnection effects in the MC model, 
the parton showering model used to simulate data, and the parton distribution functions used in the MC model. Each of these must be accounted for in the systematic uncertainty on the acceptance.

\section{Jet Energy Scale}

The uncertainty on the the jet energy corrections are described in Sec. 3.2.2. These corrections are designed to properly scale the energy of jets measured by the CDF detector. Since we select events with 4 jets each with $E_{T}$ above $20 \mathrm{GeV}$, shifts in the jet energy affect the acceptance. To evaluate this uncertainty, we shift the jet energies in the signal MC up and down by the one $\sigma$ uncertainty for each of the jet energy corrections individually (these relative uncertainties are shown in Fig. 3.2). We do not shift the jet energies in the background since the dominant background is the data based QCD multijet background. We then reperform the event selection to account for the new jet energy scale and remeasure $\sigma_{t \bar{t}}$ with the new set of events. This measurement is done separately for all 6 jet energy corrections (see Sec. 3.2.2). For each correction, the up and down JES shifts cause the cross section to move in opposite directions from the nominal value, so we take the uncertainty to be half the total difference between the two shifted values. The total jet energy scale uncertainty is taken as the quadrature sum of the uncertainty from all 6 jet energy corrections giving an uncertainty of 0.6 pb..

\section{Initial and Final State Radiation}

Next, we consider acceptance effects from initial and final state radiation (ISR and FSR). Increasing or decreasing the amount of radiation from either the initial quarks or gluons involved in the $t \bar{t}$ pair production (initial state radiation) or the final daughter particles of the $t \bar{t}$ decay (final state radiation) can change the acceptance by changing the jet multiplicity of the event or the energy of the jets if the extra radiation falls within the cone of an existing jet. The amount of ISR and FSR is estimated from selected Drell-Yan events (dilepton production at hadron colliders mediated by a virtual $\gamma$ from $q \bar{q}$ annihilation) [81. Drell-Yan events are ideal for this measurement since they are 
produced by $q \bar{q}$ annihilation (like most $t \bar{t}$ pairs at the Tevatron) and since the dilepton final state is color neutral, it will not provide any quark or gluon radiation (FSR). This means that any jets in the events are likely evidence of initial state radiation. We thus measure the amount of ISR in these events as a function of the dilepton invariant mass and extrapolate the result and its uncertainty to the top quark mass scale [82].

Since ISR and FSR are the same process occurring at different stages of the event, we assume that they occur at the same rate, and we vary the amount of each simultaneously (I/FSR). To estimate the systematic uncertainty as a result of the I/FSR uncertainty, we use Pythia $t \bar{t}$ MC samples where the amount of ISR and FSR is increased or decreased by its $1 \sigma$ uncertainty. These samples are generated with PYTHIA and are otherwise identical to the standard $t \bar{t}$ MC samples used for the measurement. By replacing the standard $t \bar{t} \mathrm{MC}$ with these new samples and reperforming the $\sigma_{t \bar{t}}$ measurement, we estimate the uncertainty from ISR and FSR. We find that both the up and down samples have a lower acceptance than the nominal MC sample in the 4 jet bin, so both cross section measurements are greater than the nominal value. As a result, we take the uncertainty to $0.5 \mathrm{pb}$ from half the greatest shift from the nominal value.

\section{Color Reconnection}

Color reconnection interactions between quarks and gluons also affect the overall acceptance by changing the jet acceptance. The nominal PyтhiA MC samples we use are made with PYTHIA version 6.2 and are tuned to match Tevatron data (tune A) [83]. This PythiA model does not include color reconnection effects defined as color interactions of the $t \bar{t}$ daughter particles with the quarks from the $p \bar{p}$ collision which do not participate in the initial interactions. A new version of PYTHIA (version 6.4) has been developed which includes a model to include these color reconnection effects, and new Pythia tunes have since been developed which include data from LEP (tunes designated with the "pro" suffix) [84]. MC samples have been generated with this new version of PYTHIA using these new tunes. One sample is generated with tune "Apro" which is similar to "tune A" used in the nominal $t \bar{t} \mathrm{MC}$ samples and does not include 
color reconnection effects. The other is generated with tune "ACRpro" which is identical to tune "Apro" but has the color reconnection model turned on. To estimate the systematic uncertainty on the $t \bar{t}$ cross section from color reconnection effects, we reperform the cross section measurement using these new MC samples in place of the nominal $t \bar{t}$ MC. Since the standard $t \bar{t}$ MC used in the analysis is generated with "tune A" rather than "tune Apro", we do not compare the cross sections measured for the systematic uncertainty with the nominal value. We simply take the difference in the measurement using the "Apro" and "ACRpro" tune samples which is $0.4 \mathrm{pb}$ to be the systematic uncertainty on the $t \bar{t}$ cross section measurement.

\section{Parton Distribution Functions}

The acceptance is also affected by the PDF's used in the MC generation to account for the fraction of proton and anti-proton momentum carried by the quarks or gluons involved in the interaction. The standard $t \bar{t} \mathrm{MC}$ is made with the CTEQ5L PDF set [85. To measure the uncertainty from the PDF's, we account for three different effects: the uncertainty on the parameters in the PDF set, the uncertainty on the value of $\Lambda_{Q C D}$, and the uncertainty in the fraction of $t \bar{t}$ events produced through $g g$ fusion rather than $q \bar{q}$ annihilation. The uncertainty from each effect is measured by reweighting the signal MC events based on a different PDF set and refitting for $\sigma_{t \bar{t}}$. To measure the effect of the uncertainty on the parameterization of the PDF set, we reweight events based on 20 pairs of orthogonal eigenvectors of the CTEQ6M PDF set [86]. The L and M in the CTEQ name refers to the PDF's being calculated to leading order (L) or nextto-leading order $(\mathrm{M})$. Each eigenvector pair accounts for a $\pm 1 \sigma$ effect on a different parameter in the PDF set. For each eigenvector, we take half the total shift between the plus and minus result as the uncertainty. We sum this uncertainty in quadrature over all 20 eigenvector pairs to measure the total uncertainty on the PDF parameters. As a cross check, we also compare the $t \bar{t}$ cross section measurements made with the standard CTEQ5L PDF set and with events reweighted based on the MRST72 PDF set. These two sets use the same value of $\Lambda_{Q C D}$ so any difference in these measurements is due to differences in the parameterization of the PDF sets. We observe no difference 
in the central values of $\sigma_{t \bar{t}}$ so we take the uncertainty from the 20 CTEQ eigenvectors as the first component of the PDF systematic uncertainty on $\sigma_{t \bar{t}}$.

Next, we measure the uncertainty due to a shift in $\Lambda_{Q C D}$. To do this, we reweight the $t \bar{t}$ MC events based on results from two different MRST PDF sets [87] with different values of $\Lambda_{Q C D}$. The MRST72 weighting scheme is built with a $\Lambda_{Q C D}$ value of $228 \mathrm{MeV}$ while the one with MRST75 is built with a $\Lambda_{Q C D}$ value of $300 \mathrm{MeV}$. The cross section measurement is performed with each set of reweighting events, and the difference in the result is taken as the uncertainty from $\Lambda_{Q C D}$.

Finally, we measure the uncertainty from the percentage of $t \bar{t}$ events produced via $g g$ fusion. As discussed in Sec. 1.3 , although leading order calculations estimate the $t \bar{t}$ production from $g g$ fusion to be $95 \%$, next-to-leading-order calculations estimate that the contribution could rise as high as $20 \%$ [17]. Since the $\sigma_{t \bar{t}}$ theoretical calculation is dependent on the gluon PDFs, we include the systematic uncertainty on the $t \bar{t}$ cross section in the PDF systematic uncertainty. In the nominal $t \bar{t} \mathrm{MC}$ used in this analysis, $5 \%$ of the $t \bar{t}$ events are produced through $g g$ fusion. We reweight the MC events to scale the percentage of $g g$ fusion events up to $20 \%$ and reperform the cross section measurement. The difference between this result and the nominal result is taken as the uncertainty on the percentage of events from $g g$ fusion. When added in quadrature, the three uncertainties described above give a total systematic uncertainty from the PDF's of $0.5 \mathrm{pb}$.

\section{Parton Showering}

Finally, we consider acceptance effects from the model used to shower partons in the MC. The nominal $t \bar{t}$ signal MC uses Pyтнia to model the showering. To measure the uncertainty, we replace the $t \bar{t}$ MC with a similar sample generated using the HERWIG MC generator [55] which uses a different model to handle the parton showering. Usually, the uncertainty is taken to be the total difference in the results from the two models. However, we found an $\sim 20 \%$ difference between the two measurements. Typically, this uncertainty is $\sim 3 \%$ in other analysis (like the one in [88]). We investigated this effect and found that it comes from a $20 \%$ larger rate of jets faking $\tau$ 's in Herwig. Upon 
further investigation, detailed in App. E, we found that the HERwiG fake rate did not match what was observed in the data. We measured the acceptance in PYTHIA and HeRwig $t \bar{t}$ MC and found that when we required the reconstructed $\tau$ to match a generated $\tau$ in the MC there was a $6 \%$ acceptance difference between the two generators. As a result, we assign a $6 \%$ systematic uncertainty from the parton showering model giving a systematic uncertainty on the measurement of $\sigma_{t \bar{t}}$ of $0.5 \mathrm{pb}$.

\subsubsection{Efficiency Scale Factor Uncertainty}

Although the tagging and trigger SF's have been included in the description of the acceptance, we separate out their uncertainties as part of the uncertainty on the $\epsilon$ terms in Eqn. 4.3. We also consider the lepton identification SF uncertainty here as well.

\section{b-tagging Scale Factor}

Recall, we find that the MC does not perfectly model the b-tagging rates in the data as described in Sec. 4.3. To account for this, we then apply tagging scale factors for heavy and light flavor jets to to properly weight the MC to match the data. The $S F_{b t a g}$ for jets matched to $b$ quarks is $0.95 \pm 0.05$ [76]. For $c$ quarks, we use the same scale factor, but we double the uncertainty. To measure the uncertainty due to heavy flavor tagging, we reweight events by shifting the tagging scale factor by its uncertainty and reperforming the analysis. The uncertainty is half the difference between the shifted measurements. We do this separately for jets matched to $b$ and $c$ quarks, and sum the two uncertainties in quadrature to quote the total systematic uncertainty due to heavy flavor tagging. We cite a separate uncertainty for mistagging, the rate of b-tagging light quark jets. For jets matched to light flavor, the uncertainty on the mistag rate is measured to be $20 \%$ [76]. To measure the effect of this uncertainty on the $t \bar{t}$ cross section, we shift the mistag rate in the MC up and down by its $20 \%$ uncertainty and reperform the cross section measurement. The quoted uncertainty is half the difference between the shifted measurements. We evaluate the b-tagging uncertainty to be 0.4 $\mathrm{pb}$, however, the mistag uncertainty is only $0.1 \mathrm{pb}$. 


\section{Trigger Scale Factor}

Next we account for the uncertainty from the $S F_{\text {trigger }}$. As is discussed in Sec. 3.1. we use level 1 and level 2 scale factors to model the trigger in the MC. To measure the trigger efficiency uncertainty, we shift these scale factors by their uncertainties, and redo the measurement. The systematic is taken to be half the difference between the shifted measurements. Evaluated to be $0.1 \mathrm{pb}$, this uncertainty is one of the smaller uncertainties on the measurement.

\section{$\tau$ Identification Scale Factor}

Finally, we measure the uncertainty from the $\tau$ identification scale factor. As described in Sec. 4.3, the $\tau$ identification scale factor is measured to be $0.95 \pm 0.22$ [75]. To measure the uncertainty from lepton ID, we shift this scale factor up and down by its uncertainty and redo the measurement. The uncertainty is taken to be half of the difference between the shifted measurements. We find the $\tau$ identification systematic uncertainty on $\sigma_{t \bar{t}}$ to be $0.2 \mathrm{pb}$.

\subsubsection{Background Uncertainty}

There are two sources that contribute to the background systematic uncertainty. The first source is the K-factor heavy flavor correction for $W+$ heavy flavor MC. The second is the uncertainty on the fraction of QCD events measured in the data.

\section{$W+$ Heavy Flavor K-factor}

The $W+$ heavy flavor MC K-factor correction described in Sec. 4.3 has been measured to be $1.5 \pm 0.3[80]$. The uncertainty on the k-factor translates directly into an uncertainty on the number of predicted background events which in turn causes an uncertainty on the $t \bar{t}$ pair production cross section. To measure this uncertainty, we shift the K-factor up and down by its uncertainty and reperform the measurement. We take half the total difference in the shifted measurements as the uncertainty. We find this uncertainty to be $0.1 \mathrm{pb}$. 


\section{QCD Multijets fraction}

The second contribution to the background uncertainty is the fraction of QCD multijet events in the data. To evaluate this we use data events which are selected without a $E_{\mathrm{T}}$ requirement which we find are heavily dominated by QCD multijet events. Recall in Sec. 4.2, we compared the distributions of variables in these events to events from the QCD multijet model to validate the model. We find that the QCD multijet model generally agrees very well with the data. The shape of the NN output is used to fit the contribution of QCD multijet events in the data, therefore we compare the distribution of the NN output between the QCD multijet model and the data to measure the uncertainty on the QCD multijet contribution.

To do this we consider the shape of the NN output in data and in the QCD multijet events selected without the $E_{\mathrm{T}}$ or NN selection requirements. We normalize these distributions to unit area and compare the shapes for data and QCD multijets with and without the b-tagging requirement. We then measure the ratio of these two shapes as a function of the NN output. Figure 5.2 shows the shapes of the NN output as well at the ratio of data to QCD multijet contribution in each bin. We fit this ratio with a $1^{\text {st }}$ order polynomial. For the pretag selected events, we keep the fit below a NN output value of 0.7 as this region is QCD multijet dominated and has very little signal contribution (see Fig. 4.10). For the b-tagged selected events, we move this limit to a NN output of 0.6 as the signal contribution is larger in the b-tagged events (see Fig. 4.11. We take the uncertainty on the slope to represent a $\pm 1 \sigma$ uncertainty on the QCD multijet fraction in the data. To measure the resulting uncertainty on the fraction of QCD multijet events, we develop two reweighting schemes for the QCD multijet events that shift the output of the $\mathrm{NN}$ by the fit slope plus or minus its uncertainty. As an extreme case cross check, we also determine maximum and minimum reweighting schemes for the QCD multijet shape by defining lines that run along the upper and lower edge of the ratio (see Fig. 5.2). This gives us 4 different reweighting scheme for the QCD multijet NN output shape with and without the b-tag requirement. By reweighting the QCD multijet NN output distribution and rerunning the QCD multijet 


\begin{tabular}{|l|c|c|}
\hline Reweighting & Pretag Fraction & b-Tagged Fraction \\
\hline Maximum & 0.816 & 0.618 \\
Up & 0.748 & 0.477 \\
Nominal & 0.729 & 0.445 \\
Down & 0.664 & 0.272 \\
Minimum & 0.618 & 0.184 \\
\hline
\end{tabular}

Table 5.3: Measured QCD multijet fractions from pretag and b-tagged fits using different reweighting schemes. The up and down values are used to measure the QCD multijet fraction uncertainty. The maximum and minimum values are used as an extreme case cross check. We choose half the total shift between the up and down samples as the systematic uncertainty on the fraction of QCD multijet events. As a result, we assign an uncertainty of $4 \%$ on the fraction before the b-tagging requirement and an uncertainty of $10 \%$ on the fraction with the b-tagging requirement.

fit in the background estimation described in Sec. 4.3, we measure how different QCD multijets shapes would change the QCD multijets contribution prediction. To test this, we simultaneously shift the QCD multijets distributions with and without b-tagging. The resulting QCD multijets fractions are listed in Tab. 5.3.

To check the validity of these reweighting schemes, we reweight the QCD multijet NN output shape and compare it that of the data in Fig. 5.3. We observe that both with and without b-tagging, the maximum up and maximum down shapes clearly do not agree with the data shape. Instead, the up and down shifted reweighting schemes tend to agree much better with the NN output shape in the data. As a result, we take the QCD multijet uncertainty from the up and down shifted reweighted results as the $\pm 1 \sigma$ uncertainty on the QCD multijet fraction. We find that a $1 \sigma$ shift of the QCD multijets fraction uncertainty corresponds to change in the QCD multijet fraction of $4 \%$ and $10 \%$ without and with b-tagging, respectively.

To measure the result of this uncertainty on the top pair production cross section, we shift the QCD fraction by this uncertainty and redo the cross section measurement. The uncertainty is taken as half the difference between the two shifted measurements which gives an uncertainty of $1.7 \mathrm{pb}$. At a $20 \%$ relative uncertainty, this is the largest systematic uncertainty on the measurement of $\sigma_{t \bar{t}}$. The uncertainty is largely driven by the statistical uncertainty on the shapes of the data and QCD multijet distributions, 
Pretag Neural Network Output Shape

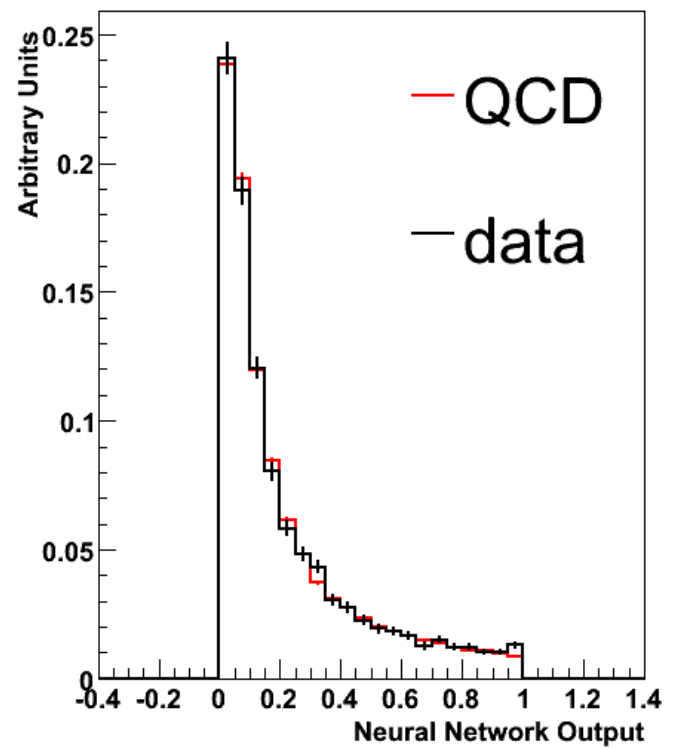

B-tagged Neural Network Output Shape

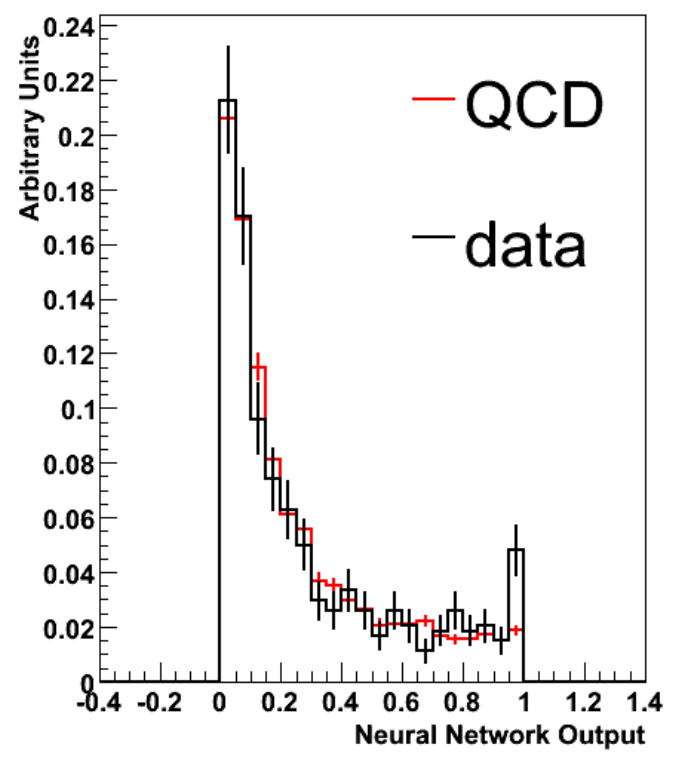

Pretag Neural Network Output Ratio

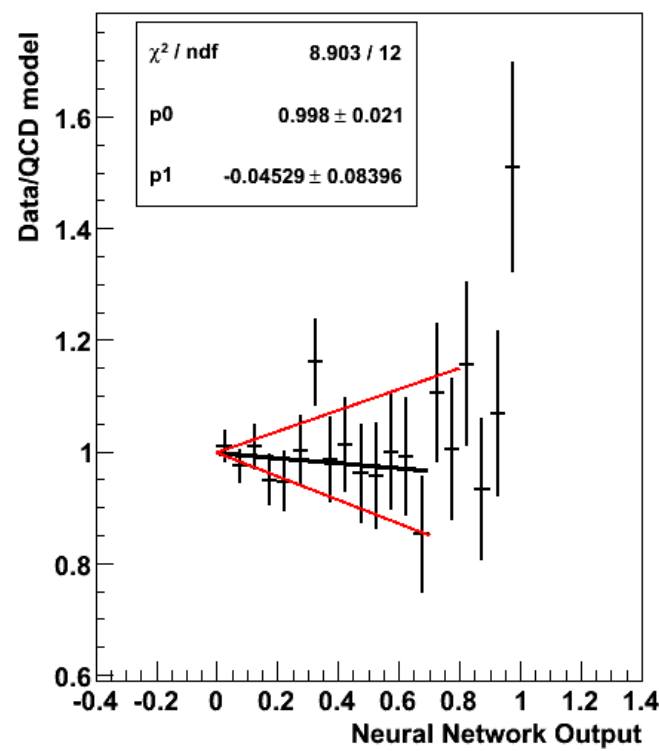

B-tagged Neural Network Output Ratio

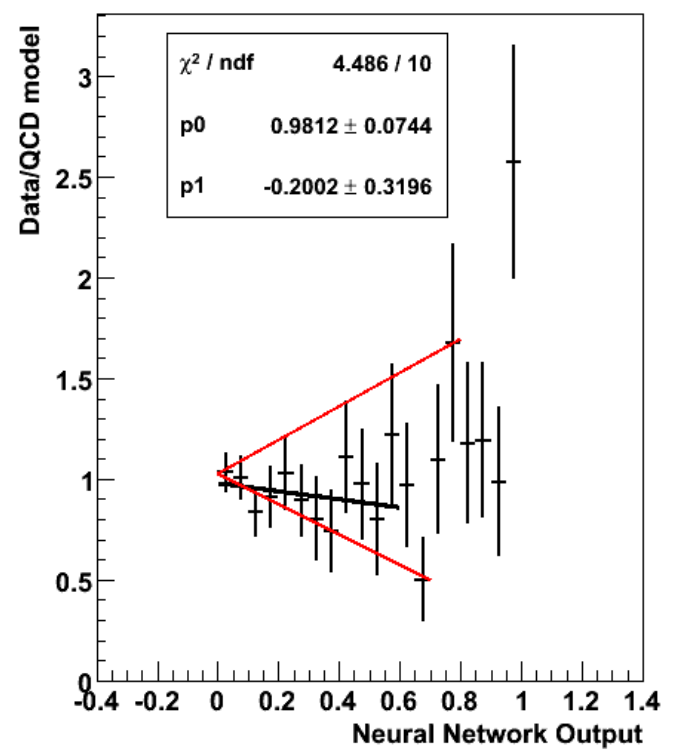

Figure 5.2: Shapes of the QCD multijet and data NN outputs without the $E_{\mathrm{T}}$ requirement. The top row is before the b-tagging requirement, and the bottom row is after the b-tag requirement. On the left, the shapes are normalized to 1 . On the right is the ratio of data to QCD multijets for each bin. The black line is from a 1st order polynomial fit. The red lines were chosen to give a maximum possible shift for use as an extreme case cross check. 

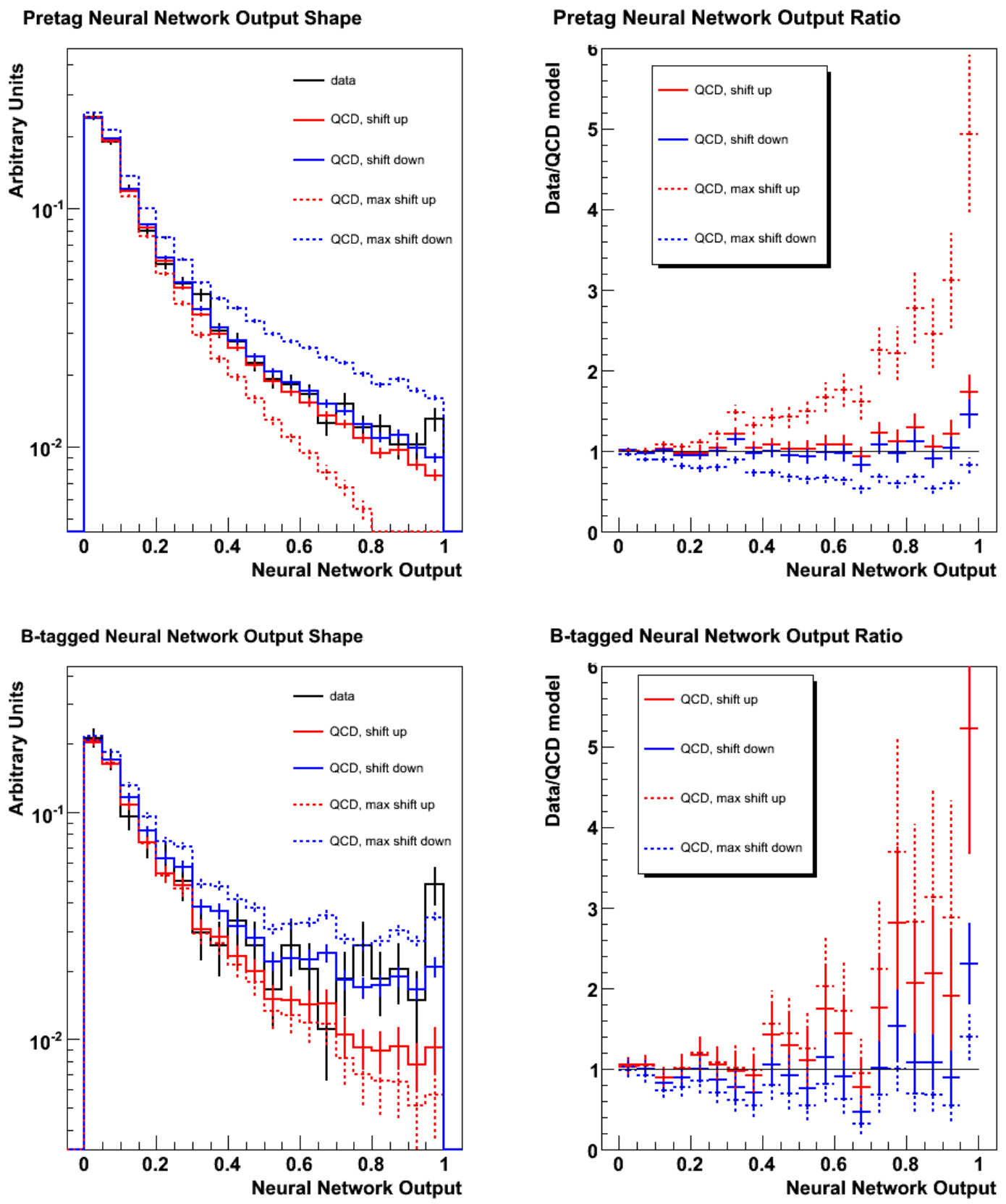

Figure 5.3: On the left is the NN output and the QCD multijet NN output from each of the 4 reweighting schemes given in Tab. 5.3. The ratio of the data to the QCD multijet contribution in each bin from all 4 schemes is shown on the right. The top row shows the results before the b-tagging requirement while the bottom row shows the results after the b-tagging requirement. The solid red and blue lines are the QCD multijet shape after reweighting with the fit line from Fig. 5.2. The dashed red and blue lines are the QCD multijet shape after the maximum/minimum extreme cases reweighting are applied to the QCD multijet shape. 
since we found very good agreement between the two shapes. As a result, this systematic uncertainty is expected to scale as a statistical uncertainty with an increase in data.

\subsubsection{Luminosity Uncertainty}

Based on the CLC accuracy and the uncertainty on the theoretical cross section of inelastic $p \bar{p}$ scattering (both described in Sec. 2.2.6), we measure the uncertainty on the luminosity of the data to $6 \%$ [48]. As a result, we take a $6 \%$ systematic uncertainty on the measured $t \bar{t}$ cross section to account for the uncertainty of measured luminosity. A $6 \%$ effect on the measured cross section gives a systematic uncertainty of $0.5 \mathrm{pb}$.

\section{5 $\sigma_{t \bar{t}}$ Result with Systematic Uncertainty}

Finally, we measure the top pair cross section in the hadronic $\tau+$ jets channel with 2.2 $\mathrm{fb}^{-1}$ of data to be:

$$
\begin{gathered}
\sigma_{t \bar{t}}=8.7 \pm 3.3 \text { (stat.) } \pm 2.2 \text { (syst.) pb } \\
\sigma_{t \bar{t}}=8.7 \pm 4.0 \mathrm{pb}
\end{gathered}
$$

assuming a top quark mass of $172.5 \mathrm{GeV}$.

Each of the systematic uncertainties along with the total systematic uncertainty of $2.2 \mathrm{pb}$ is given in Tab. 5.2. We find the $t \bar{t}$ cross section as measured in the $\tau+$ jets decay channel to be consistent with the CDF average of $7.5 \pm 0.5$ measured with 4.6 $\mathrm{fb}^{-1}$ of data. 


\section{Chapter 6}

\section{Top Mass Analysis Method}

Top quark mass measurements are usually either done as a template analysis or as a matrix element analysis. For this analysis, we use the later. Below, we describe the pros and cons of each method.

In a template analysis, the distributions in the data of certain variables which are dependent on the top quark mass are compared to MC templates of the distributions for different top quark masses. Generally, these analyses use the invariant mass of the reconstructed top quarks and $W$ bosons, but other variables such as the $P_{T}$ of the lepton produced in a lepton + jets $t \bar{t}$ decay could also be used. The mass measurement is made by determining which MC templates best fit the data distributions. In such an analysis, the templates can be constructed rather quickly; however the process of building PE's can be very time consuming. These analyses look at distributions over a large sample of events, so small anomalies in certain events are generally averaged out if the general behavior of the distribution is well understood for background events. As the measurement is built around specific variables, these methods do not use all the information available in the event to measure the top quark mass.

A matrix element analysis uses a more mathematically rigorous method to determine the top quark mass by using all possible information contained in the events. Generally, for each event, a probability distribution dependent on $M_{t o p}$ is calculated from the full kinematics of the event and the matrix element for $t \bar{t}$ production and decay (see Sec. 1.2. The individual event probabilities are then combined in a likelihood function from which the top quark mass is computed. Due to the mathematical calculation of the probability function, each event in a matrix element method can take a long time to process. However, once the probability function is determined for all events, 
building PE's is a very quick process. This offers greater flexibility in customizing the final details of a matrix element analysis method such as the window used to fit the likelihood function. Due to the maximal use of the event information in this method, matrix element measurements of the top quark mass in the lepton + jets decay channel have historically given the lowest uncertainty and tend to have the strongest weight in the combination of the top mass measurements from several different analyses and decay channels, and therefore these measurements lead the top quark mass world average. This analysis is the first such measurement of the top quark mass in the hadronic $\tau+$ jets decay channel.

We measure the top quark mass in the $\tau+$ jets decay channel using a matrix element analysis. The method uses a likelihood function based on signal and background probabilities for each event which is described in Sec. 6.2. The use of a background probability to describe the likelihood that an event is coming from a process other than $t \bar{t}$ decay also makes this analysis unique at CDF. The signal probability is calculated by integrating over the differential cross section for $t \bar{t}$ pair production based on the L.O. Mahlon-Parke $t \bar{t}$ matrix element [89] which will be described in Sec. 6.3.1. The background probability, described in Sec. 6.3.2 is likewise calculated based on the differential cross section for $W+4$ parton $(W+4 \mathrm{p})$ production which is calculated using the VecBos MC 90] algorithm's $W+4$ p matrix element. The two are combined with a signal fraction term which is constrained in the likelihood function and which is calculated from the estimation of the signal and background contributions given in Tab. 4.4

Since jet energies do not map exactly to the energy of the original hadronizing quark, for the signal probability, we calculate the differential cross sections using quark energy and angles from the integration. With the help of certain assumptions which will be described in Sec. 6.3 and the integrated quantities, we solve the event kinematics up to a quadratic equation for the neutrino solution. The signal probability is summed over the four possible solutions to this equation. To map the measured jet energies and angles to the same original quark variables, we use a set of jet-parton transfer functions which will be described in Sec. 6.4. In the case of the background probability, we 
instead numerically integrate the differential cross section using a range of likely quark energies which are derived from the measured jet energies and the parton-jet transfer functions. This calculation will be described in Sec. 6.3.2.

Additionally, to improve the energy resolution of the $\tau$ lepton, we develop a new method to reconstruct the $\nu_{\tau}$ from the $\tau$ decay. This method is described in Sec. 6.1.

Finally, we will describe the fitting algorithm used to extract the top quark mass measurement from the likelihood function in Sec. 6.5, the calibration of the measurement in Sec. 6.6, the expected statistical uncertainty in Sec. 6.7, and the results of the measurement in Sec. 6.8

\section{1 $\nu$ Scan Method for $\tau$ Lepton Reconstruction}

Events from $t \bar{t}$ decay to hadronic $\tau+$ jets, have two $\nu$ 's in the final state. One $\tau$ flavor $\nu$ comes from the leptonic $W$ decay, and another $\tau$ flavor $\nu$ results from the hadronic $\tau$ decay. For clarity sake, we will refer to the $\nu$ from the $W$ decay as $\nu_{W}$ and the $\nu$ from the $\tau$ decay as $\nu_{\tau}$. The reconstruction of a $\tau$ lepton as described in Sec. 3.2.1 only accounts for the hadronic components of the decaying $\tau$, therefore we must account for the $\nu_{\tau}$ to fully reconstruct the $\tau$ lepton. To do this, we devise a new method presented here to predict the 4-momentum of the $\nu$ 's.

First, we consider what we know about the $\nu$ 's and the $t \bar{t}$ event. The $\nu$ 's have very small mass, so we can safely assume that they are massless in the reconstruction. Thus, to fully reconstruct the 4-momentum of both $\nu$ 's in the $t \bar{t} \rightarrow$ hadronic $\tau+$ jets decay, we need the energy of both $\nu$ 's as well as the $\theta$ and $\phi$ angles of their flight. We also know that if we reconstruct the $t \bar{t}$ decay, we can constrain the $\tau$ and $W$ mass to 1.8 $\mathrm{GeV}$ and $80.4 \mathrm{GeV}$, respectively. We turn to $\mathrm{MC}$ simulation to evaluate the angles of two $\nu$ 's. Since the $\tau$ lepton from the $W$ decay tends to be fairly energetic, we find that it is boosted, and as a result its daughter particles generally decay within a very narrow cone. As is seen in Fig. 6.1, the $\theta$ and $\phi$ angles of the $\nu_{\tau}$ are very similar to the $\theta$ and $\phi$ angles of the reconstructed hadronic $\tau$ components. Additionally, the $\nu_{W}$ usually carries more energy than the $\nu_{\tau}$, and as a result, the $\phi$ angle of the $E_{\mathrm{T}}$ tends 
to be close to the $\phi$ of the $\nu_{W}$. As Fig. 6.2 shows, we find that the $\phi$ of the $\nu_{W}$ is almost always within 1 radian of the $\phi$ of the $E_{\mathrm{T}}$. Unfortunately, we have no handle to constrain the $\theta$ angle of the $\nu_{W}$.
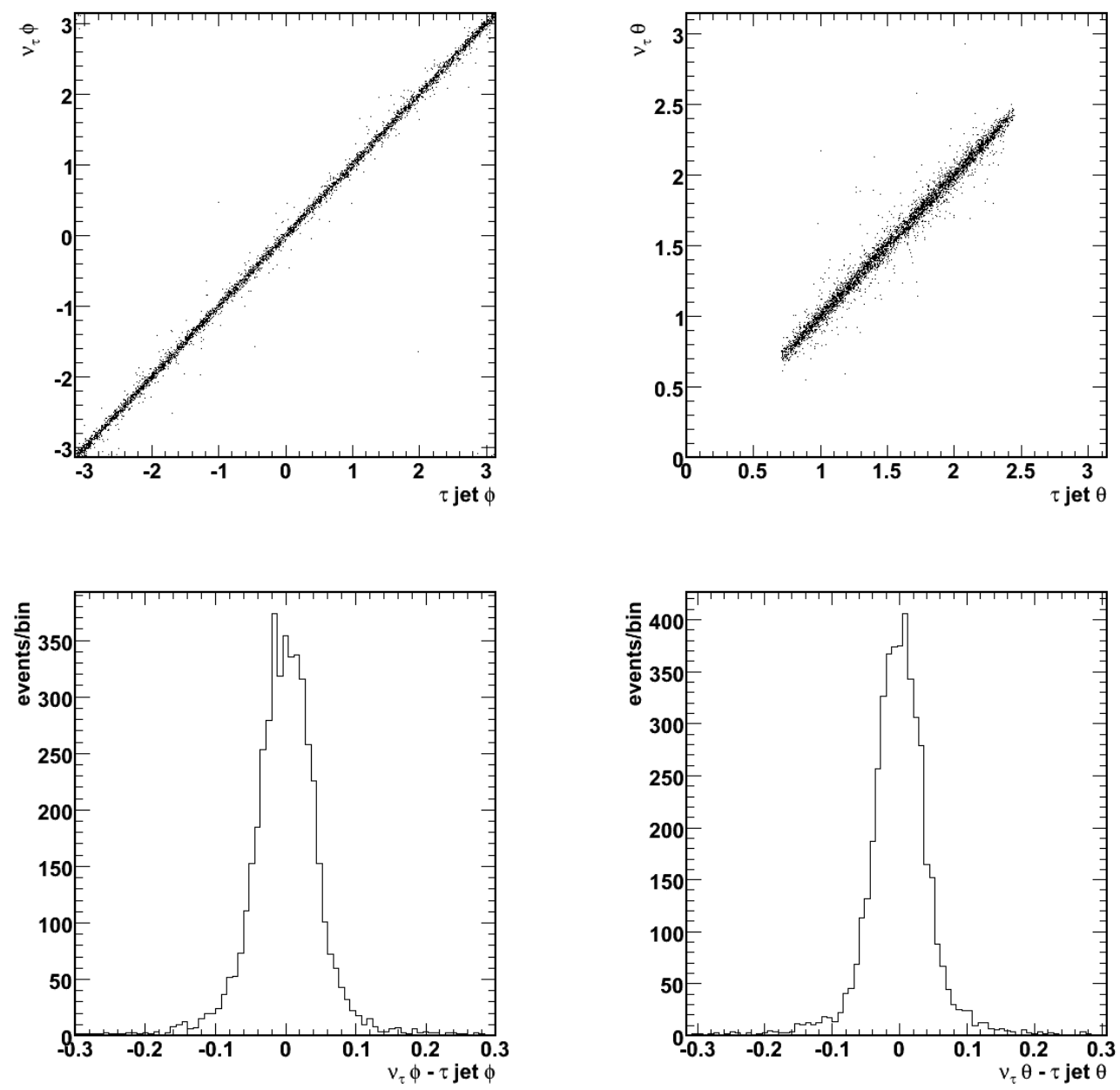

Figure 6.1: The $\phi$ (left) and $\theta$ angles (right) for the reconstructed hadronic $\tau$ compared to the generated $\nu_{\tau}$. Above shows the two angles in relation to each other with the hadronic $\tau$ "jet" on the x-axis and the $\nu_{\tau}$ on the y-axis. Below shows the difference in the angle between the $\tau$ jet and the $\nu_{\tau}$. From these distributions, we can see that the $\nu_{\tau}$ from the $\tau$ lepton decay is almost always nearly collinear with the hadronic decay components of the $\tau$ lepton (within 0.1 radians).

Putting all this information together, we design a 4-dimensional scan over the $\phi$ and $\theta$ of each $\nu$ to determine the most probable 4-momentum of the $\nu$ 's. At each scan point, the 4 angles of the $2 \nu$ 's 4 -momentum are assumed which leaves us with only 2 


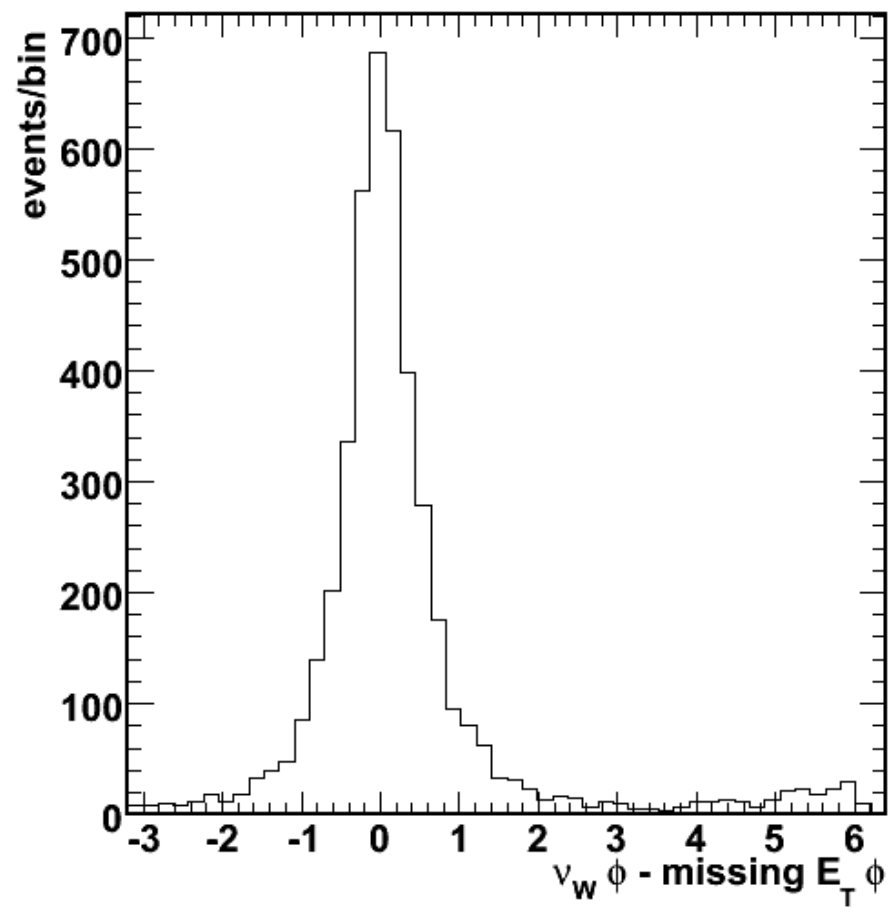

Figure 6.2: Distribution of the difference between the $\nu_{W} \phi$ and the $E_{\mathrm{T}} \phi$ observed in $t \bar{t} \mathrm{MC}$ events. We find that the $\phi$ angle of the $\nu_{W}$ is almost always within 1 radian of the $E_{\mathrm{T}} \phi$. 
unknowns, the energy of each $\nu$. We then can use the mass constraints on the $W$ and $\tau$ lepton along with the reconstructed hadronic $\tau$ 4-momentum to solve for the energy of each $\nu$. This gives a unique solution for each point in the 4 dimensional grid of angles. For the $\nu_{\tau}$, we consider potential $\phi$ and $\theta$ values which are within 0.1 radian of the reconstructed $\tau \phi$ and $\theta$. For the $\nu_{W}$, we consider $\phi$ values with 1 radian of the $\phi$ of the $E_{\mathrm{T}}$. Since we have no handle for $\theta$ for the $\nu_{W}$, it is allowed to range from 0 to $\pi$. We consider 25 points in both angles for the $\nu_{\tau}$ and 50 points for the angles of the $\nu_{W}$. This scan considers over 1.5 million combinations of the 4 angles from the $2 \nu$ 's.

With the 4-vectors of the $\nu$ 's known for each point in the scan, we sum them to predicted the $E_{\mathrm{T}}$ for each point in the scan grid. We then build the following probability function $(\mathrm{P})$ based on the predicted and measured $E_{\mathrm{T}}$ :

$$
P=\exp \frac{-\left(E_{\mathrm{T}_{x}}^{\text {scan }}-E_{\mathrm{T}_{x}^{\text {meas }}}\right)^{2}}{2 \sigma_{E_{\mathrm{T}}}^{2}} \exp \frac{-\left(E_{\mathrm{T}_{y}}^{\text {scan }}-E_{\mathrm{T}_{y}^{m e a s}}^{\text {meas }}\right)^{2}}{2 \sigma_{E_{\mathrm{T}}}^{2}}
$$

where $\sigma_{E_{\mathrm{T}}}$ is the width of a Gaussian function fit to the difference between reconstructed $E_{\mathrm{T}}$ and the $\sum E_{T}$ of the two $\nu$ 's from the truth level MC $t \bar{t}$ events shown in Fig. 6.3. We find $\sigma_{E_{\mathrm{T}}}$ to be $15 \mathrm{GeV}$ from a Gaussian fit to these variables. We choose the set of angles which returns the highest probability to be the correct set. Figure 6.4 shows the resulting selected $\phi$ and $\theta$ angles for the $\nu_{\tau}$ from the scan method vs the true angles for the same $\nu$. With $\nu_{\tau}$ reconstructed, we sum its 4-momentum with the reconstructed hadronic $\tau$ 4-momentum to recover the 4-momentum of the $\tau$ lepton before it decays. See Fig. 6.5 for the final energy resolution of the reconstructed $\tau$ lepton. While this method does a good job of reconstructing the $\nu_{\tau}$, it does not do a very good job of determining the z component of the $\nu_{W}$ 's momentum due to the lack of constraint on the $\theta$ angle of the $\nu_{W}$. As a result, we provide the reconstructed $\tau$ lepton (with $\nu_{\tau}$ added in) to the signal and background probabilities used to measure $M_{\text {top }}$ in the matrix element method, but we ignore the scan results for the $\nu_{W}$. The $\nu_{W}$ will instead be reconstructed within the calculation or the parton level kinematic solution described in App. C

The $\tau$ lepton energy resolution is still not a delta function after recovering the $\nu_{\tau}$. We test the effect this has on the Matrix Element result by running the method with 

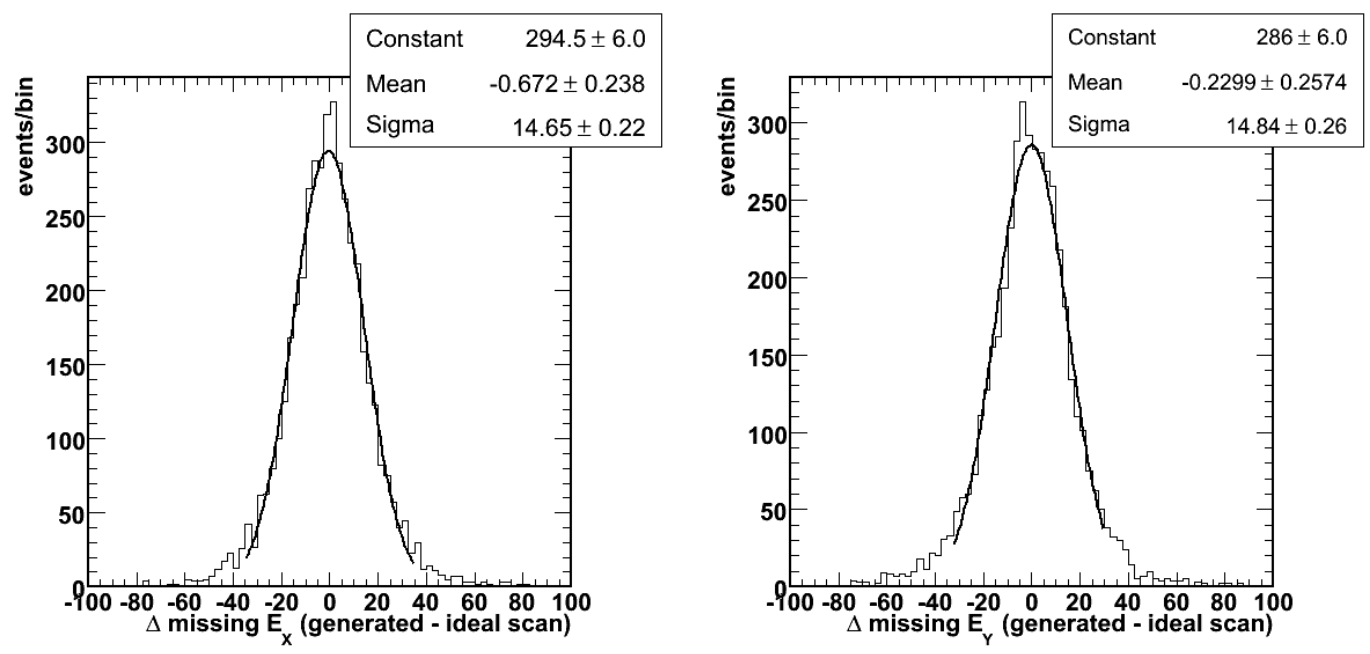

Figure 6.3: The difference between the $\sum E_{T}$ of the two $\nu$ 's (generated $E_{\mathrm{T}}$ ) and the event reconstructed $E_{\mathrm{T}}$. The $E_{\mathrm{T}}$ is broken down into its x component (left) and y component (right). Both are fitted with Gaussian functions, and the width is taken as $\sigma_{E_{\mathrm{T}}}$. Based on these fits, we take $\sigma_{E_{\mathrm{T}}}$ to be $15 \mathrm{GeV}$.

electrons which have had their energy resolution smeared by a Gaussian function fit to the energy resolution measured in $\tau$ 's. A full description of this test is given in App. B. We find that the smeared electrons return a small constant bias on the mass measurement which can be calibrated out and a slight increase in the measurement's uncertainty.

\subsection{Likelihood Function}

To extract the top quark mass from the selected events, we use a maximum likelihood estimation method. In this method, we build a likelihood function based on a probability density function which depends on certain parameters of interest. Assuming that the parameters of interest obey a Gaussian distribution, by maximizing the likelihood function for these parameters over a sample of events, we estimate the central value of the parameters for the parent distribution.

In this analysis, we maximize a likelihood function based on the probability of an event coming from a $t \bar{t}$ decay with a given top quark mass $M_{t o p}$. The likelihood function is built by multiplying the probability distribution from several events together. The 

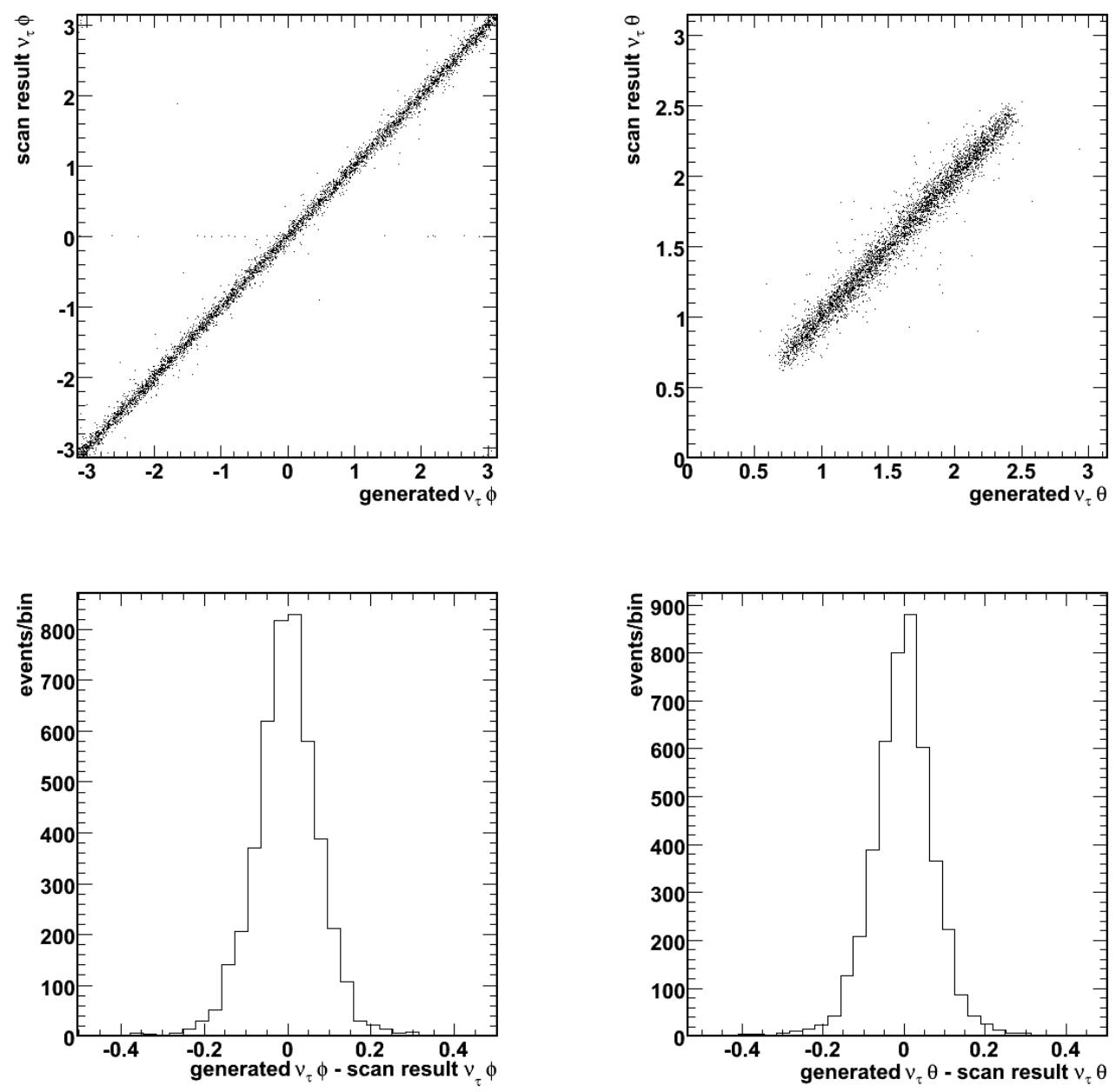

Figure 6.4: The top row shows $\phi$ (left) and $\theta$ (right) for the most probable values for $\nu_{\tau}$ from the $4 \mathrm{D}$ scan (y-axis) versus the true angles from the MC generator information (y-axis). The bottom row shows the difference between the best scan point and truth information for $\phi$ (left) and $\theta$ (right). These distributions show that the 4 dimension scan method does a good job of selecting the correct angles for $\nu_{\tau}$ with little to no observed bias. 

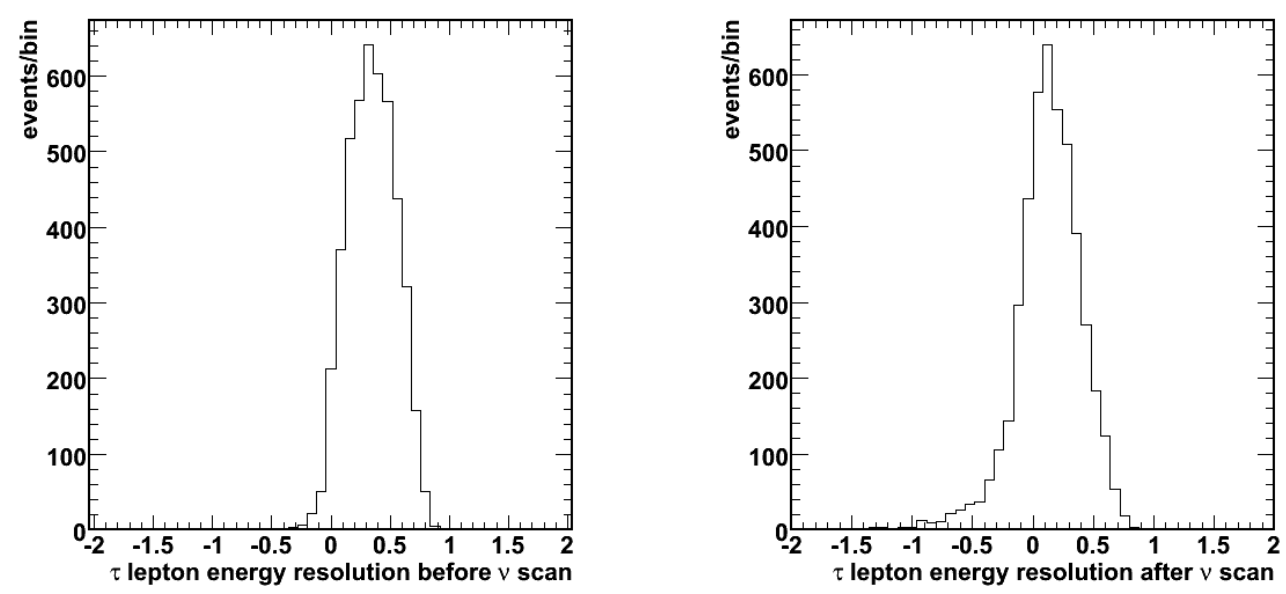

Figure 6.5: Energy resolution of reconstructed $\tau$ 's. The left distribution is made before the $\nu$ recovery algorithm. The right distribution is made after recovering the $\nu$ through the scan method. While there is still an observed offset in the reconstructed energy after the scan method, the offset is significantly reduced from the case with no $\nu$ scan.

probability distribution is based on the differential cross section from a $t \bar{t}$ decay for signal and $W+4$ p production for background. The only parameter of interest in this analysis is $M_{t o p}$. The probability distribution is written as:

$$
P=c_{s} P_{s}\left(\vec{x} ; M_{\text {top }}\right)+A_{b k g d}\left(1-c_{s}\right) P_{b k g d}(\vec{x})
$$

where $P_{s}\left(\vec{x} ; M_{\text {top }}\right)$ and $P_{b k g d}(\vec{x})$ are the signal and background probabilities, $c_{s}$ is the signal probability expected in the data which ranges from 0 to $1, \vec{x}$ represents the measured quantities from the CDF detector, and $A_{b k g d}$ is a relative normalization term used to correct differences between how the two probabilities are calculated. It is important to note that only the signal probability has any dependence on $M_{t o p}$, and for each event this probability is calculated for 31 different top quark masses from $145 \mathrm{GeV}$ to $205 \mathrm{GeV}$ in $2 \mathrm{GeV}$ steps. The background probability is a single value calculated for each event. We expect the background probability to dominate the probability function for background events which will minimize their contribution to the top quark mass measurement. 
The likelihood function, $\mathcal{L}$, is simply defined for $\mathrm{N}$ events as:

$$
\mathcal{L}=\prod_{i=1}^{N} P .
$$

However, to improve the statistical uncertainty on the top mass measurement, we add a Gaussian constraint on the background fraction $\left(1-c_{s}\right)$ to the likelihood function. The background fraction is constrained to be $0.498 \pm 0.106$ from the prediction of signal and background events shown in Tab. 4.4. The likelihood function is thus calculated as:

$$
\mathcal{L}=\prod_{i=1}^{N} P \cdot \exp \left(-\frac{1}{2}\left(\frac{\left(1-c_{s}\right)-0.498}{0.106}\right)^{2}\right)
$$

In practice, it is easier to work with the negative log of the likelihood function rather than the likelihood function itself. This way, we sum the log of the event probabilities rather than multiplying the probabilities themselves which keeps the numbers being considered within a range which can be handled by computers. Minimizing the negative log likelihood is equivalent to maximizing the likelihood function. Since the probability distribution used to build the likelihood function is expected to have a Gaussian shape around the maximum, the negative log likelihood function will have a parabolic shape around the minimum. The statistical uncertainty on the measurement is derived based on the second derivative of the parabola as is described in App. A.

Finally, in order for the maximal likelihood method to work, the probability distribution function used to create the likelihood function must be properly normalized. However, this function only needs to be properly normalized for the parameters of interest. Any other normalizations will be constant with regard to changes in the parameters of interest, and, as a result, they will have no effect on the maximization of the likelihood function. The normalization of the probability function will be described in Sec. 6.3 For this same reason, we often choose to ignore constant factors such as 2 and $\pi$ in the calculation of the signal and background probabilities. To account for differences in the scale between signal and background probabilities from differences in the overall normalization of each, we introduce a constant relative normalization term $A_{b k g d}$ which can absorb any constant term in the background probability. The calculation of the 
value $A_{b k g d}$ is described in Sec. 6.3 .3

\subsection{Event Probability Calculation}

For each event, we calculate a signal and background probability by integrating over the differential cross section $(d \sigma)$ for a $t \bar{t}$ and $W+4$ p process, respectively. The differential cross section is integrated in terms of parton level quantities $\vec{y}$, and the resulting probability is convoluted with "transfer functions" which return the probability that a measured quantity x came from a parton with quantity y. The "transfer functions" $(W(\vec{x}, \vec{y}))$ described in Sec. 6.4 are normalized for all input variables $\vec{x}$, so to normalize the probability, we must divide by the integrated cross section. This gives a probability term of the form:

$$
P=\frac{1}{\sigma} \int d \sigma(\vec{y}) W(\vec{x}, \vec{y}) .
$$

where $\sigma$ is the cross section of the process derived by fully integrating $d \sigma(\vec{y})$. Since the $t \bar{t}$ cross section depends on $M_{t o p}$, it is important to include the integrated cross section normalization term in the signal probability. However, for the background probability, the $W+4$ p production cross section has no dependence on $M_{t o p}$, so it is simply a constant which can be folded into the relative normalization term $A_{b k g d}$.

At this point, we consider the detector acceptance to accurately account for the probability observed in the data. For a single event with measured quantities $\vec{x}$, the acceptance $A c c(\vec{x})$ is either a 1 or 0 if the event passes or does not pass the selection requirements, respectively. We account for this by noting that the probabilities we measure, $P(\vec{x}, \alpha)$, from events passing the selection is related to the ideal probability $P_{x}^{\text {ideal }}(\vec{x}, \alpha)$ where $x$ is $t \bar{t}$ or $W+4 \mathrm{p}$ :

$$
\begin{gathered}
P_{t \bar{t}}\left(\vec{x}, M_{t o p}\right)=\operatorname{Acc}(\vec{x}) P_{t \bar{t}}^{i d e a l}\left(\vec{x} ; M_{t o p}\right), \\
P_{W+4 p}(\vec{x})=\operatorname{Acc}(\vec{x}) P_{W+4 p}^{i d e a l}(\vec{x}) .
\end{gathered}
$$


We integrate these probabilities over the differential space of the measure detector quantities $d \vec{x}$, assuming the probabilities themselves are correctly normalized, and we find:

$$
\begin{aligned}
\left\langle A c c_{t \bar{t}}\left(M_{t o p}\right)\right\rangle & =\int \operatorname{Acc}(\vec{x}) P_{t \bar{t}}^{i d e a l}\left(\vec{x} ; M_{t o p}\right) d x \\
\left\langle A c c_{W+4 p}\right\rangle & =\int A c c(\vec{x}) P_{W+4 p}^{i d e a l}(\vec{x}) d x
\end{aligned}
$$

where $\langle A c c\rangle$ is the average acceptance. This average acceptance is defined as the number of events passing the selection divided by the number of events generated from the process considered. We measure these average acceptances from MC simulation. For $t \bar{t}$ events, the acceptance is a function of $M_{t o p}$ since higher values of the top quark mass generally produce events with higher energy jets which are more likely to pass the selection requirements. On the other hand, the average acceptance for $W+4 \mathrm{p}$ events have no dependence on $M_{t o p}$. As a result, this acceptance is merely a constant and can be folded into the relative acceptance term $A_{b k g d}$.

The differential cross section has the form of:

$$
d \sigma=\frac{(2 \pi)^{4}|M|^{2}}{\sqrt{\left(q_{1} \cdot q_{2}\right)^{2}-m_{q 1}^{2} m_{q 2}^{2}}} d \Phi_{N}\left(q_{1}+q_{2} ; p_{1}, p_{2}, \ldots, p_{N}\right),
$$

where $\mathrm{M}$ is the matrix element for the desired process (see Sec. 1.2), $q_{1}$ and $q_{2}$ are the 4 -vectors of the incident particles, $m_{q 1}$ and $m_{q 2}$ are the masses of the incident particles, and $d \Phi$ is the differential phase space for the observed $\mathrm{N}$ daughter particles with 4 -vectors $p_{i}$ which will be described in Eqn. 6.14

Finally, we convolute the differential cross section $d \sigma(\vec{y})$ with the parton distribution functions (PDF's) to account for the fact that we do not know the momentum carried by the incident partons. The PDF's $f(\tilde{q})$ are defined as the probability that the parton carries a fraction of the longitudinal momentum of the incident proton or anti-proton between $\tilde{q}$ and $\tilde{q}+d \tilde{q}$, where $\tilde{q}$ is defined as the z component of the parton's momentum $q$ divided by the momentum of the proton or anti-proton known to be 980 $\mathrm{GeV}$. The PDF's used in this analysis are derived from fits to experimental data by 
the CTEQ collaboration [85]. For the sake of this analysis, we assume that the initial interacting partons have no transverse momentum. This is a fair assumption, however initial state radiation from the incident partons may cause the $t \bar{t}$ system to gain some transverse momentum. From MC studies shown in Fig. 6.6, we find that the $P_{X}$ and $P_{Y}$ distributions of the $t \bar{t}$ system obey Gaussian forms with means of 0 and widths $\sim 4 \mathrm{GeV}$. Ideally, we would integrate over the initial $P_{X}$ and $P_{Y}$ of the $t \bar{t}$ system with the Gaussian probability included. Unfortunately, including these extra integrals adds extra time to the computing time required to complete the analysis. As a result, we assume no $t \bar{t} P_{T}$ and deal with any consequences of this assumption in the calibration of the measurement and the initial state radiation systematic uncertainty described in Secs. 6.6 and 7.5, respectively.
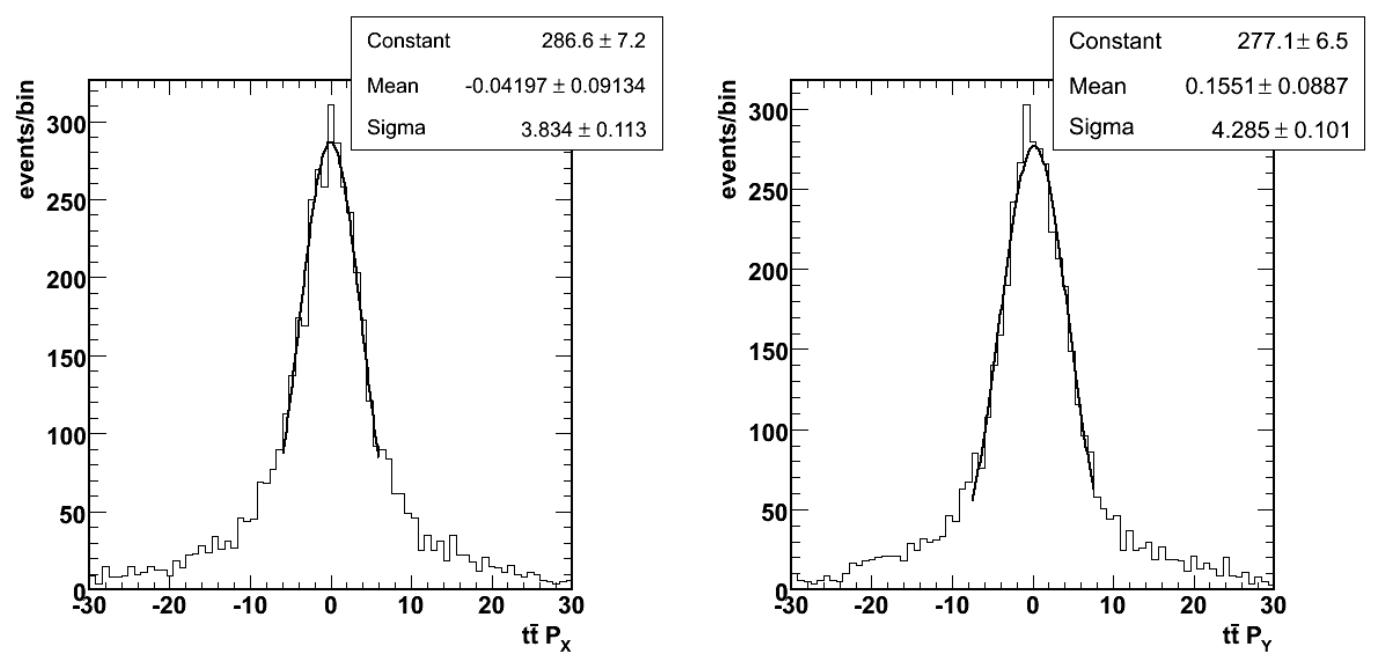

Figure 6.6: Distributions of the $P_{X}$ (left) and $P_{Y}$ (right) of the $t \bar{t}$ system in selected MC $t \bar{t} \rightarrow \tau+$ jets events. The generally low values of $P_{X}$ and $P_{Y}$ supports our assumption of no $P_{T}$ in the $t \bar{t}$ system.

With the PDFs $f(\tilde{q})$, differential cross section $d \sigma$, and transfer functions $W(\vec{x}, \vec{y})$ defined, the probability can be written as:

$$
P=\frac{1}{\langle A c c\rangle \cdot \sigma} \int \frac{(2 \pi)^{4}|M|^{2} f\left(\tilde{q_{1}}\right) f\left(\tilde{q_{2}}\right) W(\vec{x}, \vec{y})}{4 \sqrt{\left(q_{1} \cdot q_{2}\right)^{2}-m_{q 1}^{2} m_{q 2}^{2}}} d \Phi_{6} d \tilde{q_{1}} d \tilde{q_{2}}
$$

We assume that the initial state particles $q_{1}$ and $q_{2}$ are massless and have no transverse 
momentum. With this assumption, we can rewrite

$$
\sqrt{\left(q_{1} \cdot q_{2}\right)^{2}-m_{q 1}^{2} m_{q 2}^{2}}=4\left|q_{1}\right|\left|q_{2}\right|
$$

and we can rewrite Eqn. 6.11 as:

$$
P=\frac{1}{\langle A c c\rangle \sigma} \frac{(2 \pi)^{2}}{16} \int|M|^{2} \frac{f\left(\tilde{q_{1}}\right) f\left(\tilde{q_{2}}\right)}{\left|q_{1}\right|\left|q_{2}\right|} W(\vec{x}, \vec{y}) d \Phi_{6} d \tilde{q_{1}} d \tilde{q_{2}}
$$

The differential phase space for an $\mathrm{N}$ body decay, $d \Phi_{N}$, is defined as:

$$
d \Phi_{N}\left(P ; p_{1}, p_{2}, \ldots, p_{N}\right)=\delta^{4}\left(P-\sum_{i=1}^{N}\right) p_{i} \prod_{i=1}^{N} \frac{d^{3} p_{i}}{(2 \pi)^{3} 2 E_{i}},
$$

where $\mathrm{P}$ represents the 4 -vector of the colliding partons and $p_{i}$ and $E_{i}$ represent the 4 -vectors and energy of the $i^{\text {th }}$ decay daughter particle, respectively. For the 6 particle final state, this is defined as:

$$
d \Phi_{6}=\delta^{4}\left(\left(q_{1}+q_{2}\right)-\sum_{i}^{\tau, \nu, 4 q} p_{i}\right) \prod_{i}^{\tau, \nu, 4 q} \frac{d^{3} p_{i}}{(2 \pi)^{3} 2 E_{i}}
$$

where $q_{1}$ and $q_{2}$ represent the 4 -vectors of the incident partons. We use the delta function $\left(\delta^{4}\left(\left(q_{1}+q_{2}\right)-\sum_{i}^{\tau, \nu, 4 q} p_{i}\right)\right)$ in Eqn. 6.15 to compute the integrals over $d \tilde{q}_{1}, d \tilde{q}_{2}, d p_{\nu}^{x}$, and $d p_{\nu}^{y}$. However, first we must relate $d \tilde{q_{1}}$ and $d \tilde{q_{2}}$ to the quantities $q_{1}$ and $q_{2}$ in the delta function. The longitudinal fraction of the proton or anti-proton momentum carried by $q$ can be written as:

$$
\tilde{q}=\frac{q^{z}}{E_{p}}
$$

where $E_{p}$ is the total energy of the proton or anti-proton which is equal to $980 \mathrm{GeV}$. If, we assume that the incident partons carry no transverse momentum, then $|q|=q^{z}$, and we can rewrite $d \tilde{q_{1}} d \tilde{q_{2}}$ as:

$$
\begin{aligned}
d \tilde{q_{1}} d \tilde{q_{2}} & =d\left(\frac{\left|q_{1}\right|}{E_{p}}\right) d\left(\frac{\left|q_{2}\right|}{E_{\bar{p}}}\right)=\frac{1}{2 E_{p} E_{\bar{p}}} d\left(\left|q_{1}\right|+\left|q_{2}\right|\right) d\left(\left|q_{1}\right|-\left|q_{2}\right|\right), \\
& =\frac{1}{2 E_{p} E_{\bar{p}}} d\left(\left|q_{1}\right|+\left|q_{2}\right|\right) d\left(\left|q_{1}\right|-\left|q_{2}\right|\right) .
\end{aligned}
$$

With this change of variables and integration, the differential phase space now becomes:

$$
d \Phi=\frac{1}{2 E_{p} E_{\bar{p}}} \frac{d p_{\nu}^{z}}{(2 \pi)^{3} 2 E_{\nu}} \prod_{i}^{\tau, b, b, j, j} \frac{d^{3} p_{i}}{(2 \pi)^{3} 2 E_{i}}
$$


Finally, we break the 3 dimensional integrals over the jet vectors into their spherical coordinates of momentum $\rho$ and solid angle $\Omega$ :

$$
d \Phi=\frac{1}{2^{6} \cdot(2 \pi)^{18}} \frac{1}{2 E_{p} E_{\bar{p}}} \frac{d p_{\nu}^{z}}{E_{\nu}} \frac{d^{3} p_{\tau}}{E_{\tau}} \prod_{i}^{b, b, j, j} \frac{\rho^{2} d \rho_{i} d^{2} \Omega_{i}}{E_{i}}
$$

\subsubsection{Signal Probability Calculation}

For each event, recall that the signal probability is calculated for 31 different top quark mass values ranging in $2 \mathrm{GeV}$ steps from 145 to $205 \mathrm{GeV}$. Since we already know the value of $M_{t o p}$ in the $t \bar{t}$ MC samples, to save computational time with the signal MC samples, we only calculate the signal probability for 21 mass points centered around the known $M_{t o p}$ for the particular signal MC. The signal probability uses the leading order $t \bar{t}$ matrix element $(M)$ calculated by Mahlon and Parke [89]. This matrix element averages over the spin and color of the initial state partons and sums over all possible spins and color of the final state daughter particles. The matrix element is only calculated for $q \bar{q}$ annihilation to $t \bar{t}$, however, at the Tevatron, this process accounts for approximately $85 \%$ of the $t \bar{t}$ pairs produced. We do test this assumption in the systematic uncertainties by rescaling the percentage of $t \bar{t}$ events coming from $q \bar{q}$ annihilation and $g g$ fusion described in Sec. 7.7. The $t \bar{t}$ matrix element, M, is written as:

$$
|M|^{2}=\frac{g_{s}^{4}}{9} F \bar{F}\left(2-\beta^{2} \sin ^{2} \theta_{q t}\right)
$$

where $g_{s}$ is the strong coupling constant, $\theta_{q t}$ is the angle between the incident parton and the outgoing top quark in the $t \bar{t}$ center-of-mass frame, $\beta$ is the relativistic velocity of the top quark, and $F$ and $\bar{F}$ are identical form factors which describe coupling and interactions of the leptonic and hadronic decay of the top and anti-top quark. Assuming the top decays semileptonically $\left(t \rightarrow W^{+} b \rightarrow \bar{\tau} \nu b\right)$ and the anti-top decays hadronically $\left(\bar{t} \rightarrow W^{-} \bar{b} \rightarrow \bar{u}, d, \bar{b}\right)$, these form factors are written as:

$$
\begin{aligned}
& F=\frac{g_{W}}{4} \frac{\left(m_{t}^{2}-m_{\bar{\tau} \nu}^{2}\right)}{\left(m_{b \bar{\tau} \nu}^{2}-m_{t}^{2}\right)^{2}+m_{t}^{2} \Gamma_{t}^{2}} \frac{m_{t}^{2}\left(1-c_{\bar{\tau} b}^{2}\right)+m_{\bar{\tau} \nu}^{2}\left(1+c_{\bar{\tau} b}\right)^{2}}{\left(m_{\bar{\tau} \nu}^{2}-m_{W}^{2}\right)^{2}+m_{W}^{2} \Gamma_{W}^{2}}, \\
& \bar{F}=\frac{g_{W}}{4} \frac{\left(m_{t}^{2}-m_{d \bar{u}}^{2}\right)}{\left(m_{\bar{b} d \bar{u}}^{2}-m_{t}^{2}\right)^{2}+m_{t}^{2} \Gamma_{t}^{2}} \frac{m_{t}^{2}\left(1-c_{d \bar{b}}^{2}\right)+m_{d \bar{u}}^{2}\left(1+c_{d \bar{b}}\right)^{2}}{\left(m_{d \bar{u}}^{2}-m_{W}^{2}\right)^{2}+m_{W}^{2} \Gamma_{W}^{2}},
\end{aligned}
$$


where $g_{W}$ is the weak force coupling constant, $m_{i}$ and $\Gamma_{i}$ are the mass and width of particle $i$, and $c_{i j}$ is the cosine of the angle between particle $i$ and particle $j$ in the rest frame of the relevant $W$ boson. These factors are easily adapted for the case where the top decays hadronically and the anti-top decays semileptonically.

As the top quark width $\Gamma_{t}$ is much smaller than the top quark mass, we use the narrow width approximation [91] to approximate the top quark decay Breit-Wigner functions with delta functions:

$$
\frac{1}{\left(m_{\text {event }}^{2}-m_{\text {pole }}^{2}\right)^{2}+m_{\text {pole }}^{2} \Gamma^{2}} \approx \frac{\pi}{m_{\text {pole }} \Gamma} \delta\left(m_{\text {event }}^{2}-m_{\text {pole }}^{2}\right) .
$$

Using this approximation in both form factors introduces two additional delta functions into the integration over the differential phase space. These delta functions reduce the number of integrals needed to compute the signal probability from 16 to 14 which saves considerable computing time. Functionally, with this approximation, we calculate the matrix element for each event assuming the measured top quark mass is equivalent to the top quark pole mass we are considering in the signal probability. Additionally, it should be noted that the two quarks (or jets) from the hadronic $W$ decay are indistinguishable from each other. As a result, we average the form factor for the hadronically decaying top quark over the two possible assignments for the $u$ and $d$ quarks.

We calculate $\Gamma_{t}$ from the standard three body top decay expression:

$$
d \Gamma_{t}=\frac{1}{2^{7}(2 \pi)^{5}} \frac{|M|^{2}}{m_{t}}\left(1-\frac{m_{W}^{2}}{m_{t}^{2}}\right) d m_{W}^{2} d \Omega_{W} d \Omega_{b},
$$

where $\Omega_{W}$ and $\Omega_{b}$ are the solid angles of the $W$ boson and $b$ quark, respectively, by substituting in the matrix element Eqn. 6.19 and using the narrow width approximation, this gives a top decay width $\Gamma_{t}$ :

$$
\Gamma_{t}=\frac{g_{W}^{4} m_{t}^{3} \Theta}{32^{6}(2 \pi)^{3}} \frac{1-3\left(m_{W} / m_{t}\right)^{4}-2\left(m_{W} / m_{t}\right)^{6}}{m_{W} \Gamma_{W}},
$$

where,

$$
\Theta \equiv \arctan \left(\frac{\left(m_{t}-m_{b}\right)^{2}-m_{w}^{2}}{m_{W} \Gamma_{W}}\right)-\arctan \left(\frac{-m_{W}^{2}}{m_{W} \Gamma_{W}}\right) .
$$




\section{Integrating the Signal Probability}

To compute the signal probability, we first determine the assignment of the 4 measured jets to the $b$ quark from the hadronic top decay $\left(b_{h a d}\right.$ or $\left.b h\right)$, the $b$ quark from the semi-leptonic top decay $\left(b_{l e p}\right.$ or $\left.b l\right)$, and the two light quark jets from the $W$ decay $\left(j_{1}\right.$ and $j_{2}$, sometimes expressed simply as $j 1$ or 1 and $j 2$ or 2 in subscripts for the sake of simplicity). There are 24 unique possible assignments of the 4 jets, however, due to the interchangeability of the two light quark jets from the $\mathrm{W}$, we must only consider 12 possible assignments. As we require events to have at least 1 jet tagged as coming from a $b$ quark, we use this information to reduce the possible combinations down to 6 for events with a single b-tagged jet and 2 for events with two b-tagged jet. Occasionally, an event may have three b-tags. This usually comes from the $W$ boson decaying to a charm quark which is then incorrectly b-tagged. In this case, we cannot properly identify which of the three b-tagged jets are actually coming from a $b$ quark, and we consider all 12 possible combinations without using b-tagging information. For each event, we average the signal probability over all possible combinations.

For the integration, we find the large phase space of the momentum variables $\rho_{j 1}, \rho_{j 2}, \rho_{b h}, \rho_{b l}$, and $p_{\nu}^{z}$ from Eqn. 6.18 cumbersome to work with in a numerical integration. Instead, we choose to change the variables $\rho_{j 2}, \rho_{b}, \rho_{\bar{b}}$, and $p_{\nu}^{z}$ into the square of the hadronically and leptonically decaying top quark masses $\left(m_{t h}^{2}\right.$ and $\left.m_{t l}^{2}\right)$ and $W$ boson masses $\left(m_{W h}^{2}\right.$ and $\left.m_{W l}^{2}\right)$. This is done with the Jacobian transformation:

$$
J\left(\frac{m_{t h}^{2} m_{W h}^{2} m_{t l}^{2} m_{W l}^{2}}{\rho_{j} 2 \rho_{b} \rho_{\bar{b}} p_{\nu}^{z}}\right)=a_{11} a_{21}\left(a_{33} a_{44}-a_{34} a_{43}\right),
$$


where the $a_{p q}$ factors are defined as:

$$
\begin{aligned}
a_{11} & \equiv \frac{d m_{t h}^{2}}{d \rho_{b h}}=2\left(\frac{\rho_{b h}}{E_{b h}} E_{j 1}-\rho_{j 1} \cos \theta_{b 1}\right)+2\left(\frac{\rho_{b h}}{E_{b h}} E_{j 2}-\rho_{j 2} \cos \theta_{b 2}\right) \\
a_{21} & \equiv \frac{d m_{W h}^{2}}{d \rho_{j 2}}=2 \rho_{j 1}\left(1-\cos \theta_{12}\right) \\
a_{43} & \equiv \frac{d m_{W l}^{2}}{d \rho_{b l}}=2\left[\frac{d p_{\nu}^{x}}{d \rho_{b l}}\left(\frac{\rho_{\tau}}{\rho_{\nu}} p_{\nu-p_{\tau}^{x}}^{x}\right)+\frac{d p_{\nu}^{y}}{d \rho_{b \tau}}\left(\frac{\rho_{\tau}}{\rho_{\nu}} p_{\nu-p_{\tau}^{y}}^{y}\right)\right] \\
a_{44} & \equiv \frac{d m_{W l}^{2}}{d p_{\nu}^{z}}=2\left(\frac{\rho_{\tau}}{\rho_{\nu}} p_{\nu}^{z}-p_{\tau}^{z}\right) \\
a_{34} & \equiv \frac{d m_{t l}^{2}}{d p_{\nu}^{z}}=a_{44}+2\left(\frac{E_{b l}}{\rho_{\nu}} p_{\nu}^{z}-\rho_{b l} \cos \theta_{b \tau}\right)
\end{aligned}
$$

and

$$
\begin{aligned}
a_{33} \equiv & \frac{d m_{t l}^{2}}{d \rho_{b l}}=a_{43}+2 \frac{\rho_{b l}}{E_{b l}} \rho_{\tau}-2 p_{\tau} \cos \theta_{\tau b}+2\left[\frac{\rho_{b l}}{E_{b l}} \rho_{\nu}+\frac{E_{b l}}{\rho_{\nu}}\left(\frac{d \rho_{\nu} x}{d \rho_{b l}} \rho_{\nu}^{x}+\frac{d \rho_{\nu}^{y}}{d \rho_{b l}} \rho_{\nu}^{x}\right)\right] \\
& -2\left[\sin \theta_{b \tau} \cos \phi_{b l}\left(p_{\nu}^{x}+\rho_{b l} \frac{d \rho_{\nu}^{x}}{d \rho_{b l}}\right)+\sin \theta_{b \tau} \sin \phi_{b l}\left(p_{\nu}^{y}+\rho_{b l} \frac{d \rho_{\nu}^{y}}{d \rho_{b l}}\right)+\cos \theta_{b \tau} p_{\nu}^{z}\right]
\end{aligned}
$$

where all angles are defined as the angle between the two objects identified by the subscripts 11 and:

$$
\frac{d \rho_{\nu}^{x}}{d \rho_{b l}}=-\sin \theta_{b l} \cos \phi_{b l}, \quad \frac{d \rho_{\nu}^{y}}{d \rho_{b l}}=-\sin \theta_{b l} \sin \phi_{b l}
$$

We use the delta functions from the narrow width approximation (see Eqn. 6.22) in the form factors (see Eqn. 6.21) to remove the integrals over the square of the hadronic and leptonic side top quark masses. The 16 dimensional differential phase space is thus reduced to the following 14 dimensional function:

$$
\frac{1}{(2 \pi)^{18} 2^{6}} \frac{1}{2 E_{p} E_{\bar{p}}} \frac{1}{J} \frac{\rho_{b h}^{2} \rho_{j 1}^{2} \rho_{j 2}^{2} \rho_{b l}^{2}}{E_{b h} E_{b l} E_{j 1} E_{j 1} E_{\tau} E_{\nu}} d^{3} p_{\tau} d m_{W h}^{2} d m_{W l}^{2} d \rho_{j 1} d^{2} \Omega_{j 1} d^{2} \Omega_{j 2} d^{2} \Omega_{b h} d^{2} \Omega_{b l} .
$$

Unfortunately, this 14 dimension integral still requires long CPU time to calculate for all the MC events needed to validate and calibrate the measurement. As a result, we make a few more assumptions regarding the parton level quantities. First, we assume that the momentum and angles of the $\tau$ lepton are well measured by the detector, and

\footnotetext{
${ }^{1}$ the identity of the $b$ as coming from the hadronic or leptonic decay side is to be inferred by if the angle is between a $b$ quark and a light quark or a $b$ quark and a lepton
} 
we introduce a delta function $\delta^{3}\left(\overrightarrow{p_{\tau}}-\vec{p}_{\tau}^{\text {measured }}\right)$ to remove the integral $d^{3} p_{\tau}$. While the $\tau$ angles are relatively well measured by the CDF detector, the measured $\tau$ momentum has a significant resolution spread.

To test the validity of this assumption, we use a previous version of this analysis which measures $M_{t o p}$ using electron and muon + jets events. We smeared the energy resolution of electrons in selected electron + jets $t \bar{t}$ MC and studied how this effected the measurement of $M_{t o p}$. The details of this test can be found in App. B. We find that the observed energy resolution of the $\tau$ lepton results in a small constant bias in the measurement of $M_{t o p}$. Since this bias shows no dependence on $M_{t o p}$, we are comfortable using this assumption and correcting for any resulting bias in the calibration of the method described in Sec. 6.6. The hadronically decaying top quark's mass resolution is much better than that of the leptonically decaying top quark even when the lepton's energy is well measured because of the uncertainty on the measurement of the $\nu$ 's energy in the reconstruction of the leptonically decaying top quark. As a result, additional energy resolution on the leptonically decaying top quark introduced by the lepton does not have a large effect on the measurement of $M_{t o p}$.

This assumption leaves us with 11 dimensions to integrate over, however we can further reduce this by noting that the $\theta$ and $\phi$ angles of the jets are good approximations of the same angles of the parton level quarks. We find no bias in the resolution of the quark angles themselves, however, we do find a bias when we look at the angles between the two quarks from the $W$ boson decay $\left(\alpha_{12}\right)$ and between the resulting $W$ and the hadronic side $b$ quark $\left(\alpha_{W b}\right)$. This effect and how we deal with it will be further detailed in Sec. 6.4. For now, it is noted that we perform a change of the jet angle variables into a new set of variables with a Jacobian of 1 . In the new variable frame, we integrate over $\alpha_{12}$ and $\alpha_{W b}$, and we introduce delta functions to handle the other 6 angles. As a result, the 8 dimensions of the differential phase space devoted to the jet angles reduces to 2. This leaves us with 5 dimensions to integrate over: $d \rho_{1}, d m_{W h}^{2}, d m_{W l}^{2}, d \cos \left(\alpha_{12}\right)$, and $d \cos \left(\alpha_{W b}\right)$.

With the added assumptions that the top and anti-top mass are identical in each event and that the $b$ quark is massless, for any point in this integration, we reconstruct 
the full kinematics of the event up to a quadratic equation for the neutrino momentum. For the full details of this solution, see App. C.

We integrate the differential cross section using the VEGAS adaptive MC integration algorithm [92. This algorithm is based on the principle of importance sampling. Essentially, it samples more often from points in the integration space which give the largest contributions to the probability density function. For each pass of the integration algorithm over the integration space, the algorithm keeps track of a histogram of the resulting probability density function. It then uses this histogram to choose the best sampling points for the next pass. Such an algorithm will asymptotically converge on the correctly integrated function. These algorithms perform better on smooth distributions without sharp peaks. To smooth out the integrand, we make one more change of variables from $m_{W h}^{2}$ and $m_{W l}^{2}$ to $\mu_{h}$ and $\mu_{l}$ which remove the Breit-Wigner distributions from the $W$ boson in Eqn. 6.21:

$$
\int \frac{d m^{2}}{\left(m^{2}-M_{W}^{2}\right)^{2}+M_{W}^{2} \Gamma_{W}^{2}} \rightarrow \int \frac{d \mu}{M_{W} \Gamma_{W}},
$$

where $m$ is the $W$ boson mass being integrated over for the event $\left(m_{W h}\right.$ and $\left.m_{W l}\right)$ and $M_{W}$ is the $W$ boson pole mass equal to $80.4 \mathrm{GeV}[3]$. This implies the definition of $\mu$ to be:

$$
m^{2}=M_{W} \Gamma_{W} \tan \mu+M_{W}^{2}
$$

All Breit-Wigner functions have thus been removed from the differential cross section leaving a smoothly varying function for the VEGAS algorithm to integrate. We find 10000 sampling points to be enough to properly integrate the function.

Finally, we check several distributions to verify that the signal probability is correctly working. First, in Fig. 6.7, we show a histogram of the negative log likelihood of the signal probability from each event. This is calculated across several top quark mass values, therefore we choose the mass with the highest value of $P_{\text {sig }}$ from each event. Next, we show a series of mass variables using the signal probability to select the most probable assignment of the 4 jets to parton level quantities. We scan each event for the jet-parton assignment which yields the highest signal probability across all considered mass points. We then use that assignment to make distributions of the dijet mass from 
the hadronically decaying $W$ (Fig. 6.8a), the trijet mass from the hadronically decaying top (Fig. 6.8b), the mass of the $\tau$ and the $b$ from the semi-leptonically decaying top (Fig. 6.8c), and the mass of the light quark jet from the hadronically decaying $W$ and the same side $b$ (Fig. 6.8d). Due to the interchangeability of the two light quark jets from the $W$, this final distribution has two entries per event. We expect to see peaks in the $t \bar{t} \mathrm{MC}$ in Figs. $6.8 \mathrm{a}$ and $6.8 \mathrm{~b}$ corresponding to the $W$ boson and top quark mass (although we do not expect these peaks at the measured $W$ and top quark mass due to the jet energy resolution), respectively. Figures $6.8 \mathrm{c}$ and $6.8 \mathrm{~d}$ test the kinematics of the top quark decay. Assuming the top quark decays as $t \rightarrow W b$, there should be

a cutoff in the $t \bar{t} \mathrm{MC}$ distributions at a value of $\sqrt{M_{t o p}^{2}-M_{W}^{2}} \approx 150 \mathrm{GeV}$. For all 4 variables, the QCD multijet background contribution should be relatively flat. Both the observation of the expected distributions in $t \bar{t} \mathrm{MC}$ and the agreement between the data and MC expectation provides strong evidence that the matrix element method is correctly identifying the most probably jet-parton assignment.

\section{$t \bar{t}$ Pair Production Cross Section Top Mass Dependence}

The $t \bar{t}$ production cross section is used to normalize the signal probability calculation. This cross section has a strong dependence on the top quark mass. Since the likelihood function is insensitive to multiplicative constants, we only determine this normalization correctly up to an overall constant scale if we expect to properly normalize the probability. Rather than depend on a theoretically calculated $t \bar{t}$ pair production cross section, we fully integrate the differential cross section with the 14 dimension differential phase space from Eqn. 6.35 for 39 mass points in $2 \mathrm{GeV}$ steps between 135 and $210 \mathrm{GeV}$. As a cross check, we compare the resulting distribution to the leading order theoretical cross section and the leading order PYтнIA prediction in Fig. 6.9. The resulting distribution is consistent with both leading order calculations, so we feel confident in the signal probability calculation.

We fit a function to the resulting distribution versus $M_{t o p}$ to determine the dependence of the cross section on top quark mass and use the result to normalize the signal probability as shown in Eqn. 6.13. 


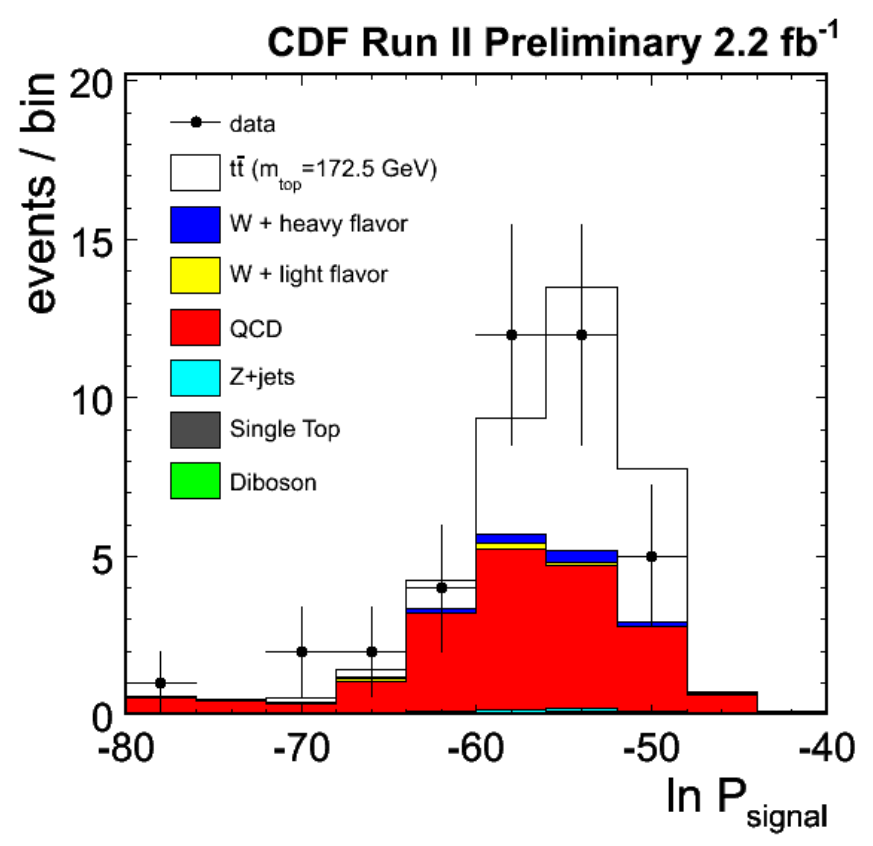

Figure 6.7: Negative log of the signal probability taken from the highest probability point along the top quark mass axis for each event. The points show the data events. The stacked histograms show the expected distribution from each process scaled to its predicted contribution. 


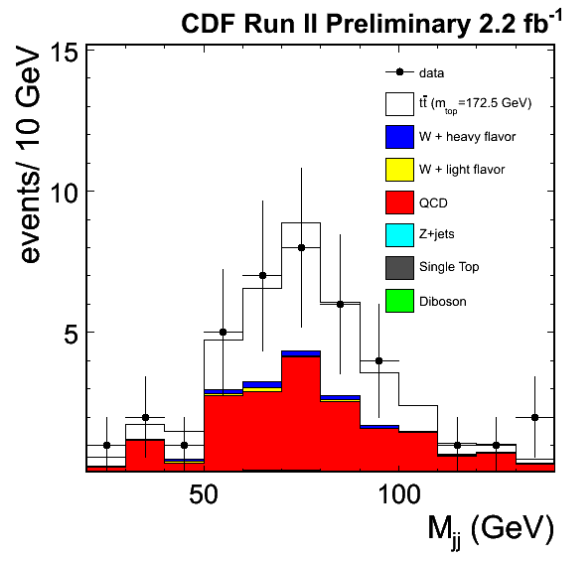

(a) $M_{j j}$

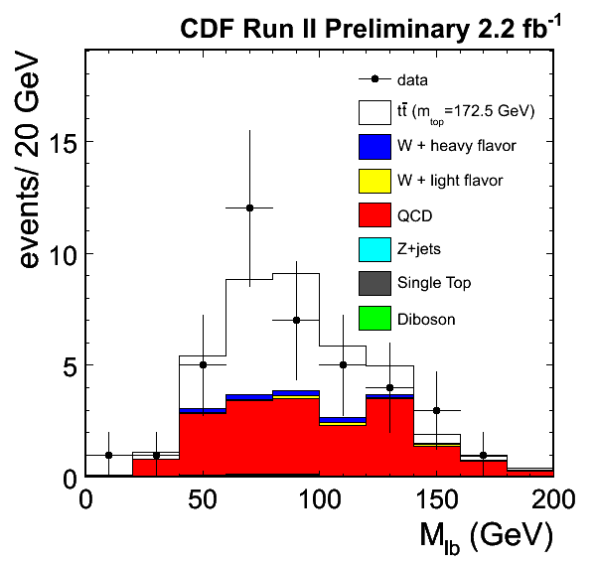

(c) $M_{l b}$

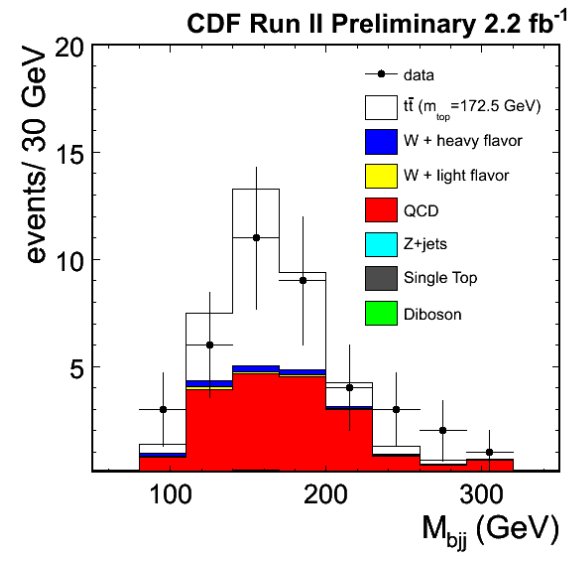

(b) $M_{b j j}$

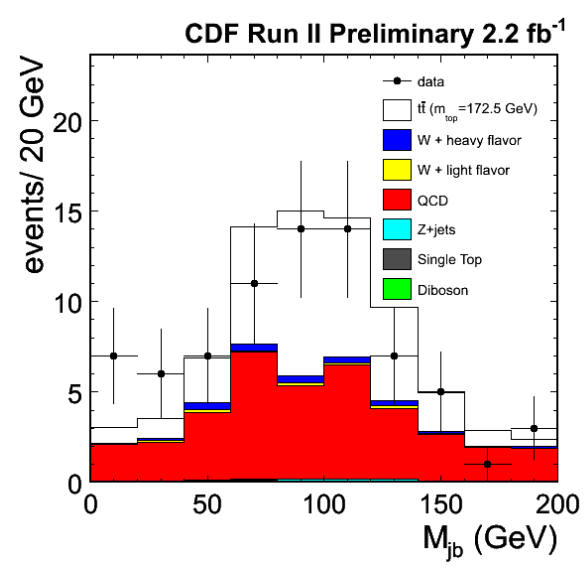

(d) $M_{j b}$

Figure 6.8: Distributions of several invariant mass variables using the signal probability term to pick out the most probable jet-parton assignment. The points show the data events. The stacked histograms give the expected distribution for all processes scaled to their predicted contribution. In Figs. (a) and (b), the $t \bar{t}$ MC peaks near the $W$ boson and top quark mass (jet energy resolution causes these variable to peak slightly below their expected values) while the QCD multijet background show no observable resonances. Figures (c) and (d) are kinematic distributions which test the hypothesis of a top quark decaying to a $W$ boson. Assuming the top quark decays to a $W$ boson and a $b$ quark, the $t \bar{t} \mathrm{MC}$ will show a cutoff at a value of $\sqrt{M_{t o p}^{2}-M_{W}^{2}} \approx 150 \mathrm{GeV}$, and the QCD multijet sample has a flat distribution as the result of the lack of any resonances in the events. It should be noted that due to the symmetry of the two jets from the $W$ boson decay, we enter both possible combinations of the $b$ quark and the light quark jets in Fig. (d) leading to two entries per event in this distribution. The observation of the expected results in the $\mathrm{MC}$ and the agreement between the $\mathrm{MC}$ expectation and the data points is strong evidence that the matrix element method is correctly identifying the most probably jet-parton assignment. 


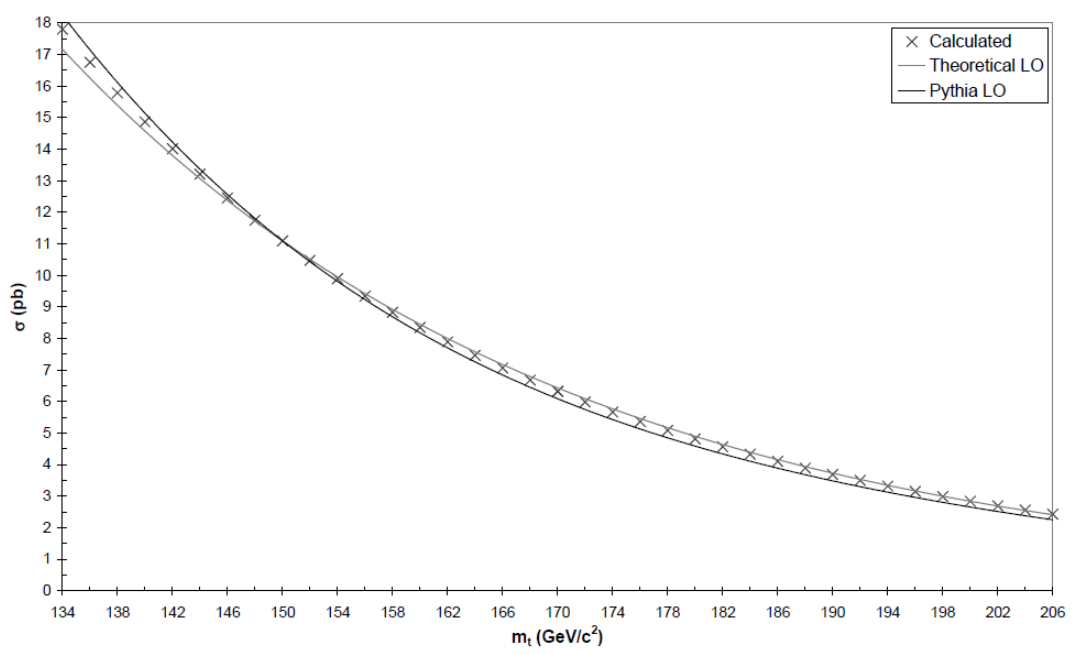

Figure 6.9: The $t \bar{t}$ pair production cross section $\left(\sigma_{t \bar{t}}\right)$ shown versus $M_{t o p}$. The crosses show the 39 calculated points from the integration of the differential cross section. For comparison, the PYTHIA leading-order cross section and the theoretical leading-order cross section are also shown in black and gray, respectively. As the shape and not the overall scale is important to the normalization of the signal probability, all three distributions are scaled to agree at an $M_{t o p}$ of $150 \mathrm{GeV}$ [93.

\section{Mean Acceptance}

The average acceptance, $\langle A c c\rangle$ in Eqn. 6.13, as a function of $M_{t o p}$ is used to normalize the signal probability, therefore we fit an acceptance function to the $t \bar{t}$ MC. First, we measure the acceptance defined as the number of events passing the selection requirements divided by the total number of $\mathrm{MC}$ events generated for several values of top mass from 150 to $200 \mathrm{GeV}$. Noting a linear dependence on $M_{t o p}$, we fit this distribution with a $1^{\text {st }}$ order polynomial. The measured $\tau+$ jets acceptance for $t \bar{t} \mathrm{MC}$ along with the fit used to normalized the likelihood function is shown in Fig. 6.10.

\subsubsection{Background Probability Calculation}

The background probability uses a similar calculation as the signal probability, but it uses a matrix element for $W+4 \mathrm{p}$ production from the VECBOs MC generator [90] as a description of the background. The choice to use a $W+4$ p matrix element to calculate the background probability is a holdover from a previous version of this analysis which 


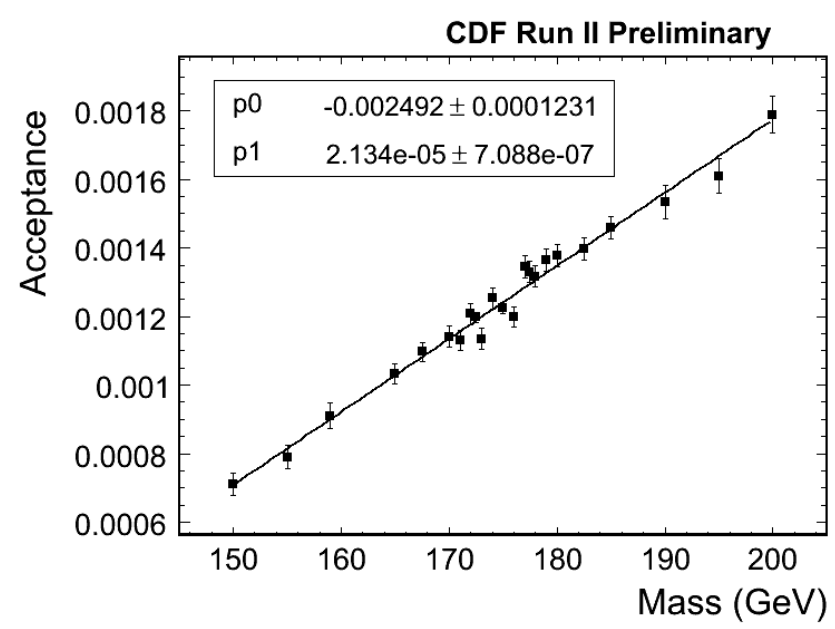

Figure 6.10: Acceptance for $t \bar{t} \rightarrow \tau+$ jets events measured from MC samples as a function of $M_{\text {top }}$ ranging from 150 to $200 \mathrm{GeV}$.

measured the top quark mass in the $e / \mu+$ jets decay channels. In these channels, the dominant background is $W+$ jets. In the $\tau+$ jets channel considered here, QCD multijet events with a jet faking a $\tau$ dominate the background events. However, this process cannot be easily and accurately described with a simple matrix element, so we chose to keep the $W+4 \mathrm{p}$ matrix element to approximate the background. Although this may not be an ideal choice for the majority of the background events, we find that, combined with the differences in the signal probability, this $W+4 \mathrm{p}$ background probability still provides reasonable separation between signal and background events as can be seen in Figs. 6.7 and 6.12 .

The background probability has no dependence on $M_{t o p}$, so for each event we calculate a single background probability. Unlike the signal probability, the average acceptance and $W+4 \mathrm{p}$ production cross section are constants with respect to $M_{t o p}$. As a result, we can ignore these factors in the likelihood function and fold them into the relative normalization term $A_{b k g d}$.

To calculate the background probability, we start with the integral over the differential cross section given in Eqn. 6.13. Unlike in the case of $t \bar{t}$ decay, we must consider all 24 possible ways to assign the 4 measured jets to the 4 produced quarks, so we must average the cross section calculation over all 24 combinations. Just as in 
the signal probability calculation, we use the 4 dimensional delta function in the differential phase space to complete the integrals over $d \widetilde{q_{1}}, d \tilde{q_{2}}, p_{\nu}^{x}$, and $p_{\nu}^{y}$. This leaves 16 dimensions to integrate in the differential phase space. Next, we use Eqn. 6.30 from the signal probability Jacobian to change the variable $d^{z} p_{n u}$ to $m_{W}^{2}$. This leaves us with the following differential phase space:

$$
d \Phi=\frac{1}{2^{6} \cdot(2 \pi)^{18}} \frac{1}{2 E_{p} E_{\bar{p}}} \frac{1}{2 \rho_{\tau}\left(\rho_{\tau} p_{\nu}^{z}-p_{\tau}^{x} \rho_{\nu}\right)} d m_{W}^{2} d^{3} p_{\tau} \prod_{i}^{4 q} \frac{\rho^{2} d \rho_{i} d^{2} \Omega_{i}}{E_{i}} .
$$

To save time in the calculation, we remove a few more of the integrals using certain assumptions. First, we use a delta function to set the $W$ mass to $80.4 \mathrm{GeV}$. By doing this however, we must sum over the two possible solutions for the $p_{\nu}^{z}$. Next, we assume that the $\tau$ 3-momentum and the quark angles are well measured by the CDF detector. Previously, in the signal probability, we found that the quark angles appear to be well measured by the CDF detector, but that there was some bias when observing the angles between partons from the decay of a parent particle. Since the partons in the background probability matrix element are not coming from a $W$ boson or top quark, we no longer need to worry about these biases, so we can safely assume all 8 angles are well measured. This leaves just the 4 integrals over the quark momenta in the differential phase space.

The Vecbos matrix element is summed over several leading order diagrams for $W$ $+4 \mathrm{p}$ production. As a result, it is more complicated to calculate than the $t \bar{t}$ matrix element and requires considerable more time to compute. For this reason, trying to integrate the background probability for all events with the VEGAS MC integration method used for the signal probability would be impossible within a reasonable time frame. Instead, we develop a custom iterative numerical integration algorithm. For each event, we use the jet energy transfer function to determine the probability distribution of the parton energy $E^{y}$ that would give rise to the measured jet energy $E^{x}$. We then use these probability distributions to select 4 parton energies and use them to calculate the background probability. We iterate this process repeatedly and average the background probability from each iteration until the result converges. As seen in Fig. 6.11, previous 


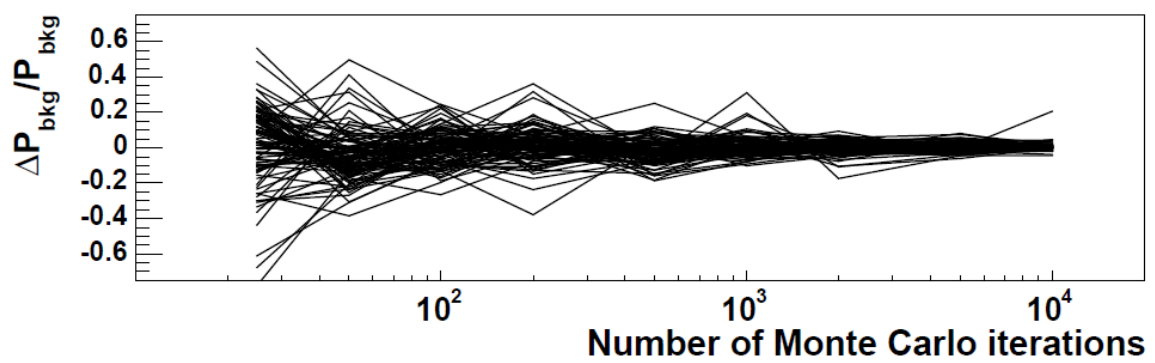

Figure 6.11: The fractional variation in the calculated background probability shown as a function of the number of calculation iterations for several different events. We find the background probability converges within 20\% after 400 iterations [94].

studies show that with 400 iterations, the resulting background probability converges within $20 \%$ 94. Since the background probability has no dependence on the top quark mass and is only used to minimize the contribution to the measurement from background events, this is a sufficiently precise calculation.

\subsubsection{Relative Normalization}

The signal and background probabilities are computed with two different methods, therefore it is possible that the two probabilities are not on the same scale. In fact, since we often ignore any constant quantities that have no $M_{t o p}$ dependence, it is highly unlikely that the two probabilities are properly scaled. We fix this by introducing a relative normalization parameter $A_{b k g d}$ which is applied to the background probability to properly rescale it. We chose the value of this parameter to minimize the uncertainty on the mass distribution. If the parameter is too large, the background probability will wash out the signal probability in signal events and the uncertainty on the $M_{t o p}$ measurement will increase. On the other hand, if the parameter is too small, the background probability will not be large enough to smooth out the fluctuations in the signal probability in background events, and the resulting fluctuations will add to the uncertainty in the measurement of $M_{t o p}$. To optimize $A_{b k g d}$, we run PE's with different values of $A_{b k g d}$ ranging from $1 \times 10^{-13}$ to $1 \times 10^{-6}$. Each PE is built with fully simulated backgrounds and $t \bar{t}$ MC signal events with a top quark mass of $172.5 \mathrm{GeV}$. Figure 6.13 


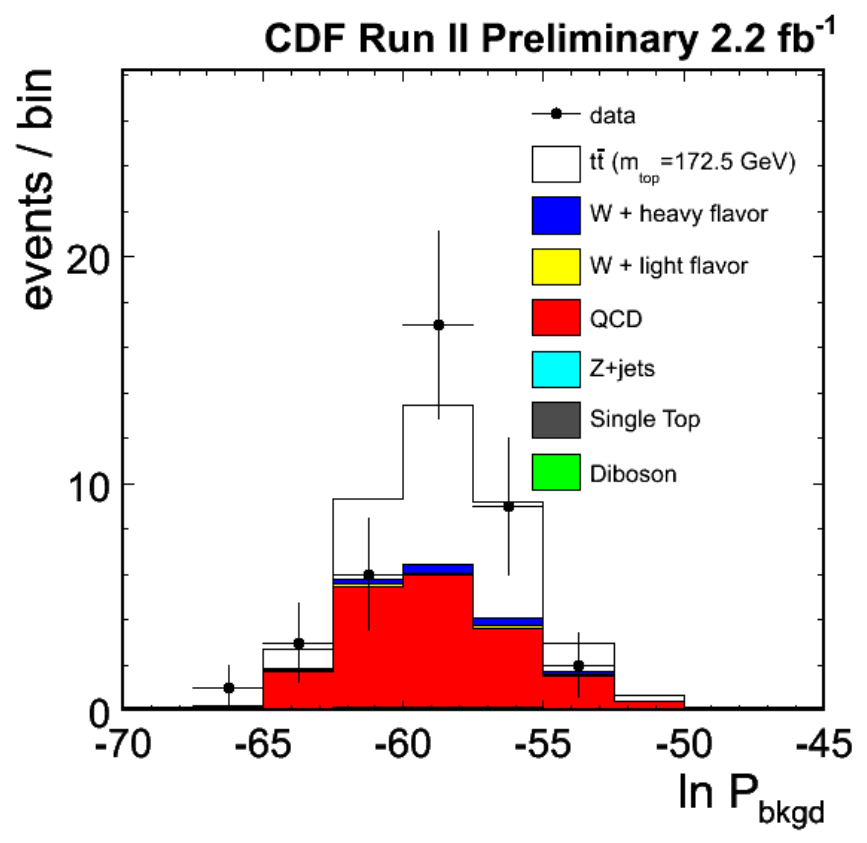

Figure 6.12: Negative log of the background probability. The points show the data events. The stacked histograms give the expected distribution based on the predicted contributions from signal and background processes. We find the data matches the MC expectation well. 
shows the distribution of the measured uncertainty as a function of $A_{b k g d}$ from these pseudoexperiments (PE's) which we use to select $10^{-9}$ to be the ideal value of $A_{b k g d}$.

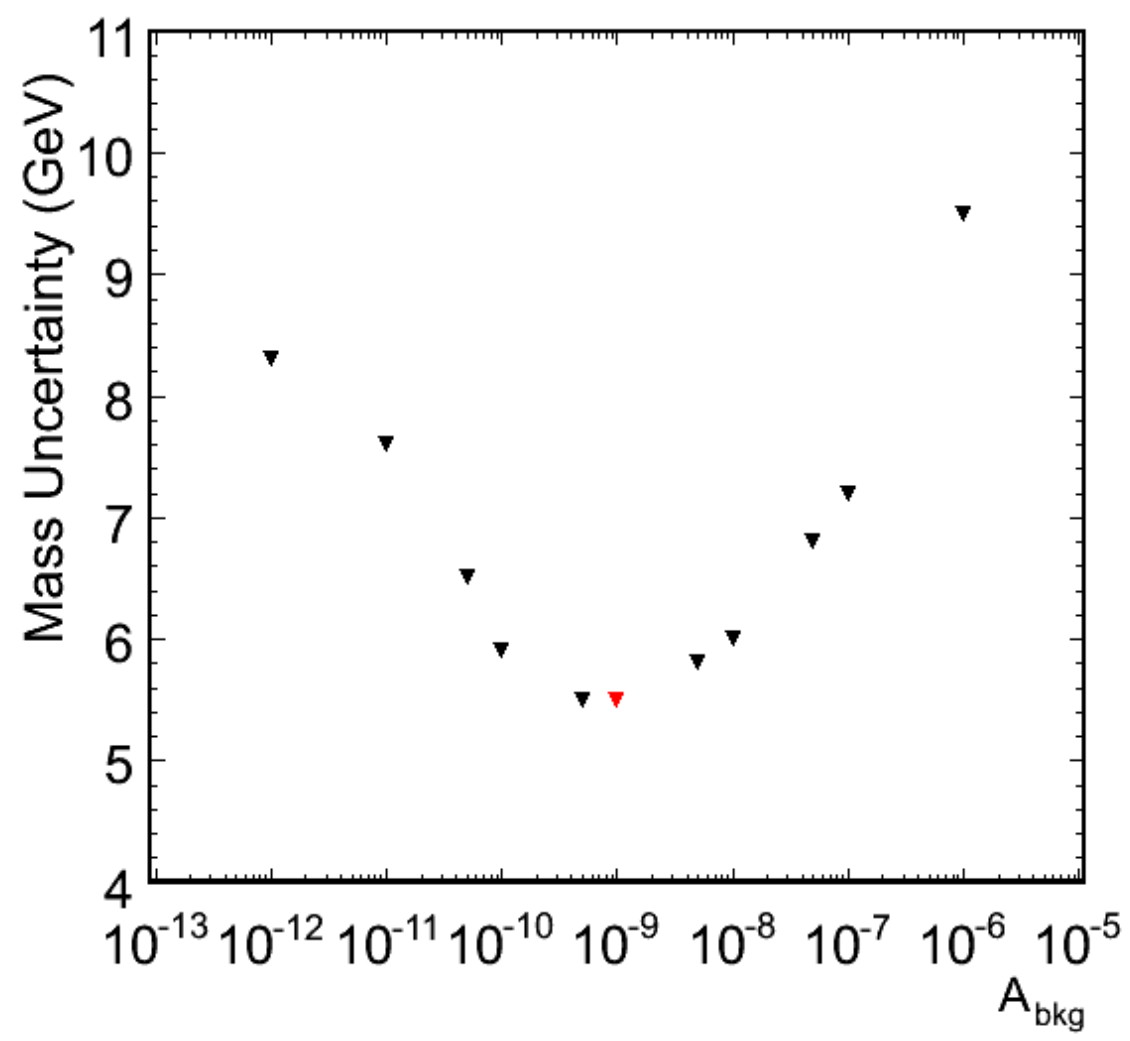

Figure 6.13: Uncertainty on $M_{\text {top }}$ for $172.5 \mathrm{GeV}$ PE's versus measured $A_{b k g}$ for $\tau+$ jets events. The red point at $1 \times 10^{-9}$ is the minimum point we chose to use for $A_{b k g d}$.

\subsection{Parton-Jet Transfer Functions}

The Transfer Function (TF) is a mapping function which is used to account for differences between quantities measured in the detector and the same quantity of the produced particle. We use notation $W(\vec{x}, \vec{y})$ to represent the TF where $\vec{x}$ refers to measured quantities and $\vec{y}$ refers to the true quantity. In this analysis, we do not actually measure the $\nu$, and we assume that the $\tau$ lepton is well measured by the detector. As a result, the only differences we have to account for in the TF are the differences between the measured jet quantities and the underlying parton quantities. We fold the delta 
function for the $\tau$ reconstruction which was used in the probability calculation into the TF which we can thus write:

$$
W_{\vec{x}, \vec{y}}=\delta^{3}\left(\overrightarrow{p_{\tau}^{y}}-\overrightarrow{p_{\tau}^{x}}\right) \prod_{i}^{4}\left(\frac{1}{\left(\rho_{i}^{x}\right)^{2}} W_{E}\left(E_{i}^{x}, E_{i}^{y}\right)\right) W_{A}\left(\overrightarrow{\Omega^{x}}, \overrightarrow{\Omega_{i}^{y}}\right),
$$

where $W_{E}\left(E^{x}, E^{y}\right)$ is the probability function for the jet-parton energy mapping and $W_{A}\left(\Omega_{i}^{x}, \Omega_{i}^{y}\right)$ is the probability function for the jet-parton angle mapping. The details of these two components will be further described in Secs. 6.4.1 and 6.4.2. As is implied in Eqn. 6.39, the energy TF's for each jet are assumed to be independent and thus are separated out for each jet. However, as will be explained in Sec. 6.4.2, the same assumption does not hold for the angular component of the TF. Both components of the TF are developed by comparing the energy and angles of matched jet-parton pairs from Pythia $t \bar{t}$ MC.

The TF are used slightly differently in the signal and background probability calculation. In the case of the signal probability, for each point in the integration, the $\vec{y}$ quantities are input into the TF along with the measured $\vec{x}$ quantities. The TF then returns a probability that a parton with quantity $y$ would produce a measured quark with quantity $x$. The differential cross section is then convoluted with the product of these probabilities at each point in the integration space for each mass point considered. This leads to the signal probability term maximizing for the most likely jet-parton assignment and top quark mass which would give an event with quantities $\vec{x}$. In the case of the background probability, we use the TF to predict the probability distribution of a possible parton level quantity $y$ that would result in a measured quantity $x$. We then randomly select values of $y$ from this distribution when iterating the calculation of the background probability for the integration algorithm.

The fit parameters of the TF's have not changed since a previous version of this matrix element analysis which selected electron and muon + jets events [53]. Since then, new signal and background MC samples have been generated with a higher luminosity profile to better match the luminosity profile observed in the data. Additionally, we are now selection $\tau+$ jet events which could potentially have a different jet energy spectrum. We check that the TF fits are still valid in the new MC and in the $\tau+$ 
jets event selection to make sure the current fits are still valid. This study is described in App. D. We find that the TF fits are still valid for the new MC and the $\tau+$ jets selection, so we do not need to refit the TFs for this analysis.

\subsubsection{Energy}

The energy component of the TF is used to account for differences between the energies of the quarks produced in the top quark decay and the resulting jets we measure in the CDF detector. Even after applying the jet energy corrections as described in Sec. 3.2 .2 , we know that the jet energies do not match the underlying parton energies. We check the distribution of $\delta$, defined as the energy difference between the jet and parton energy for pairs of matched jets and partons from $t \bar{t} \mathrm{MC}$, and we find a distribution that we can approximate with the sum of two Gaussian functions. The first Gaussian function is used to fit the peak of the function while the second Gaussian fits the tails of the distribution. The double Gaussian function requires 5 parameters: the mean of both Gaussians $\left(p_{1}\right.$ and $\left.p_{4}\right)$, the $\sigma$ of both Gaussians $\left(p_{2}\right.$ and $\left.p_{5}\right)$, and a normalization term between the two $\left(p_{3}\right)$. Each of these $p_{i}$ terms is parameterized by a constant term and a term which is dependent on the energy of the parton, $E^{y}$ :

$$
p_{i}=a_{i}+b_{i} \cdot E^{y} .
$$

The energy TF thus takes the form:

$$
W_{E}\left(\delta, E^{y}\right)=\frac{1}{2 \pi\left(\left|p_{2}\right|+\left|p_{3}\right|\left|p_{5}\right|\right)}\left(e^{\frac{-\left(\delta-p_{1}\right)^{2}}{2 p_{2}^{2}}}+\left|p_{3}\right| \cdot e^{\frac{-\left(\delta-p_{4}\right)^{2}}{2 p_{5}^{2}}}\right),
$$

where the normalization term $\frac{1}{2 \pi\left(\left|p_{2}\right|+\left|p_{3}\right|\left|p_{5}\right|\right)}$ comes from the normalization requirement $\int W_{E}\left(\delta, E^{y}\right) d^{3} \vec{p}^{x}=1$.

We find that jets from $b$ quarks obey a different distribution than jets from light flavor quarks, so we split these two samples up and fit transfer functions for each independently. In the signal probability calculation, we determine whether to use the $b$ quark or the light flavor quark energy TF based on which type of quark the jet is assigned to based on the particular jet-parton assignment being considered. The background probability is based on a $W+4$ parton process with no explicit heavy 
flavor quarks, so in this case, we use the light flavor quark transfer function with all 4 jets.

We determine the $a_{i}$ and $b_{i}$ parameters by fitting the energy TF to the distribution of $\delta$ for well matched jet-parton pairs. These pairs are taken from approximately 60000 Pyтhia $t \bar{t}$ MC events which pass the event selection and where all 4 jets are matched to quarks from the $t \bar{t}$ decay within an $\eta-\phi$ cone of size 0.4 . Additionally, thanks to the $\eta$ dependent jet energy correction described in Sec. 3.2.2, the mean value of $\delta$ is generally constant as a function of $\eta$. However, we do find that the spread of $\delta$ increases as the jet $\eta$ increases. As a result, we separate the jet-parton pairs into 8 different bins in $\eta$. The $\eta$ bin sizes are non-constant and are chosen so that each bin contains approximately 15000 jet-parton pairs. The 8 bins are: $0.0 \leq|\eta|<0.15,0.15 \leq|\eta|<0.30,0.30 \leq|\eta|<0.45$, $0.45 \leq|\eta|<0.62,0.62 \leq|\eta|<0.82,0.82 \leq|\eta|<1.10,1.10 \leq|\eta|<1.50,1.50 \leq|\eta|$.

The resulting TF fits compared the energy resolution, $\frac{E_{\text {jet }}-E_{\text {parton }}}{E_{\text {parton }}}$, can be seen for light flavor quarks in Fig. 6.14 and $b$ quarks in Fig. 6.15.

\subsubsection{Angle}

Generally, the $\theta$ and $\phi$ angles of the jets are very good measurements of the same angles of the underlying partons. When we check the resolution functions of these angles from the two light flavor quarks from the $W$ boson decay in Fig. 6.16, they all appear to be nearly delta functions. We see similar behavior in the angles of the $b$ quarks. However, when we attempt to reconstruct the $W$ boson mass using the energy of the two quarks from the MC combined with the angles of the matched jets, we observe a bias (see Fig. 6.17). When the $W$ boson decays into 2 massless particles, its mass is calculated as: $m_{W}^{2}=2 E_{1} E_{2}\left(1-\cos \theta_{12}\right)$, where $E_{i}$ is the energy of the $i^{t h}$ particle and $\theta_{12}$ is the angle between the two daughters. Since we take $E_{1}$ and $E_{2}$ directly from the parton information in the $\mathrm{MC}$, a bias in the $W$ mass must be coming from a bias in the measured angle between the two jets. To learn more, we check the resolution distribution of angle between the two light quark jets and find that it is skewed. From Fig. 6.18 we see that the measured jets tend to be slightly closer together than the actual partons. This occurs because of the color connections between the two light 

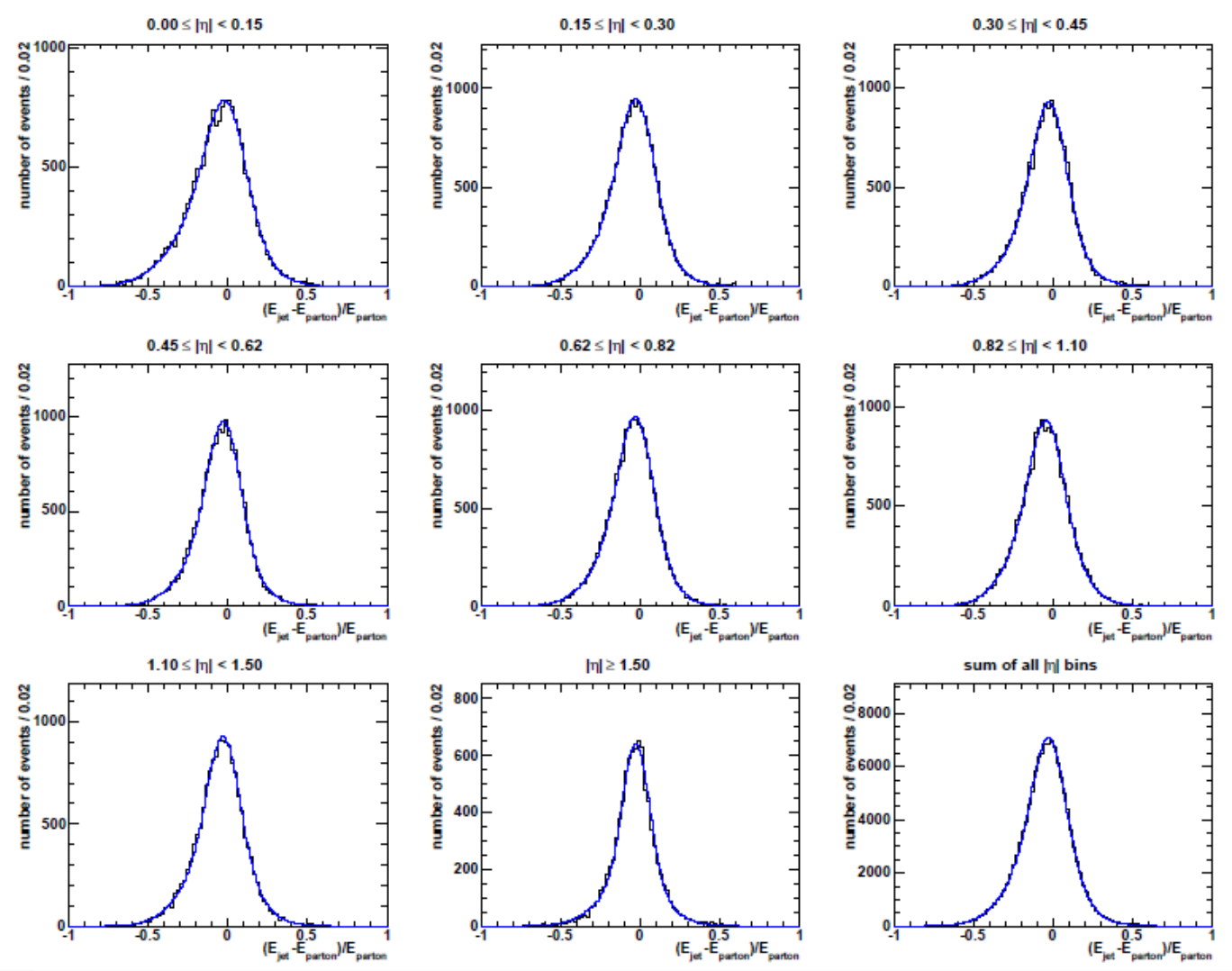

Figure 6.14: Energy resolution for jet-parton pairs, defined as $\frac{E_{\text {jet }}-E_{\text {parton }}}{E_{\text {parton }}}$, from light flavor quarks. The distribution is shown for each of the $8 \eta$ bins and finally with all all bins combined. The blue line shows the TF fit used in the analysis [93]. 

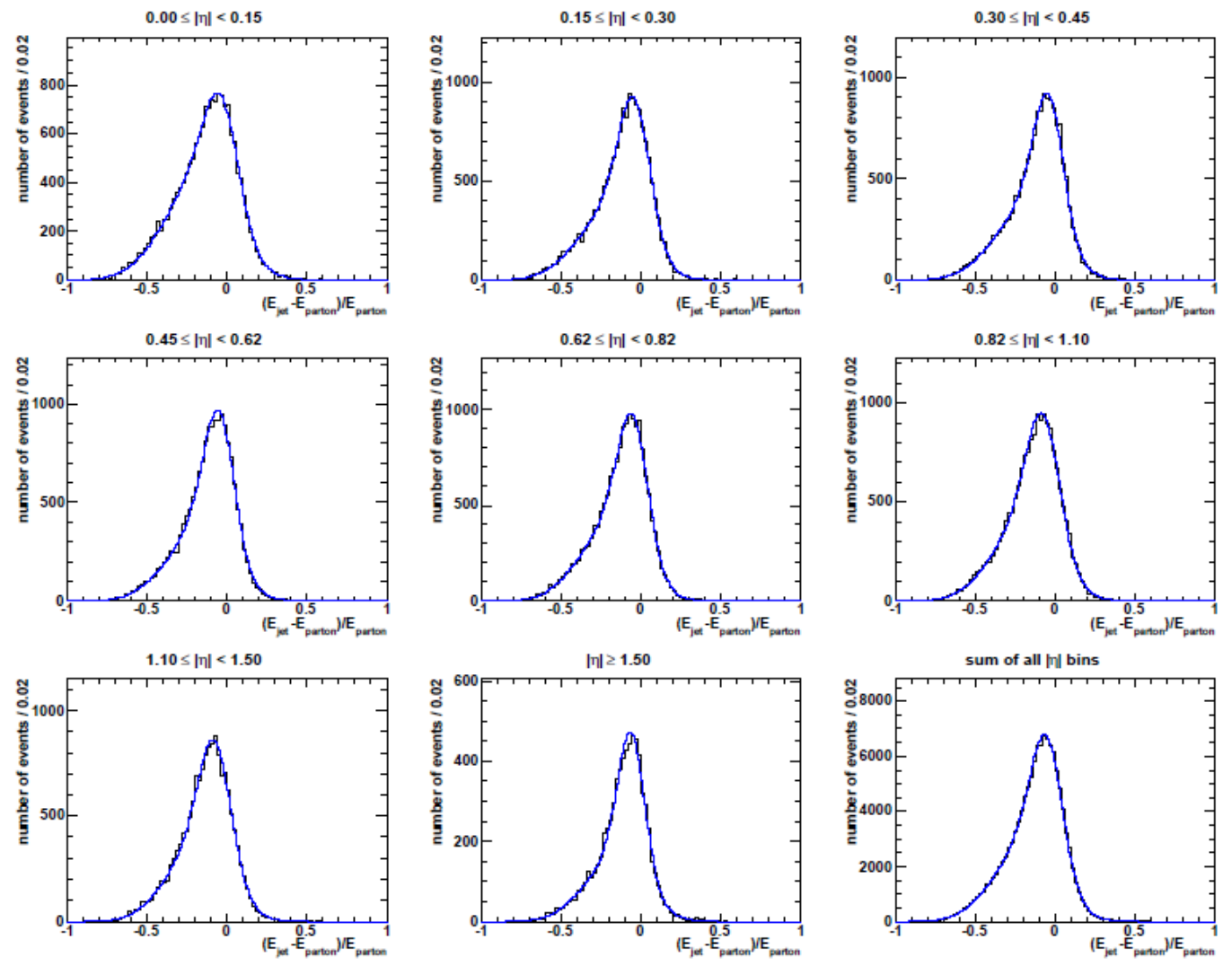

Figure 6.15: Energy resolution for jet-parton pairs, defined as $\frac{E_{\text {jet }}-E_{\text {parton }}}{E_{\text {parton }}}$, from $b$ quarks. The distribution is shown for each of the $8 \eta$ bins and finally with all all bins combined. The blue line shows the TF fit used in the analysis [93]. 
flavor quarks from the $W$ boson decay. The $W$ is a color singlet, therefore the two light flavor quarks into which it decays have opposite color charge. These quarks thus share a color interaction which causes their radiation as they hadronize to slightly skew towards each other. This leads to the jets from these quarks being reconstructed slightly closer together than the actual produced quarks. This effect is consistent with the lack of a bias observed in the resolution of each angle individually. Since the $W$ boson can decay in nearly any direction within the full $4 \pi$ solid angle (the only limitations are based on the jet selection requirements we place on the solid angle), this skewness is distributed randomly and equally in all directions around a single jet's angles. This causes the resolution in the measurement of an angle to spread, but it does not cause any observable bias. By the same reasoning, we find a smaller but similar effect in the angle between the $b$ quark and $W$ boson from the hadronic top quark decay.

We believe these biases come from color interactions between daughter particles of the hadronically decaying $W$ and top quark, therefore any such effects would be negligible in the matrix element for the background probability. As a result, we do not use the angular TF in the calculation of the background probability. For that calculation, we assume that the quark angles are well measured by the corresponding jet angles.

In the signal probability, we develop an angular TF to correct for this skewness. First, we perform a change of variables of the angles into a coordinate system in which the angle between the two light quark jets is easily parameterized. This is done by rotating the coordinate axis in which the $\hat{z}$ axis is normal to the direction of the two light quark jets. In this new coordinate plane, the angle between the two jets can be easily defined as an angle in the $\mathrm{x}-\mathrm{y}$ plane. We thus change the angular variables from $\theta_{1}, \theta_{2}, \phi_{1}$, and $\phi_{2}$ to the $\theta$ and $\phi$ angles which define the rotation of the axis, $\alpha_{12}$ the angle between the two light flavor quark jets, and $c_{12}$ the angle which defines the bisector of the angle between the two light quark jets. We chose to use the angle of the bisector because it remains constant even when the two jets skew closer together. For details regarding the exact equations used to in this change of variables, see [93]. The thing to note is that the Jacobian for this change of variables is equal to -1 which 

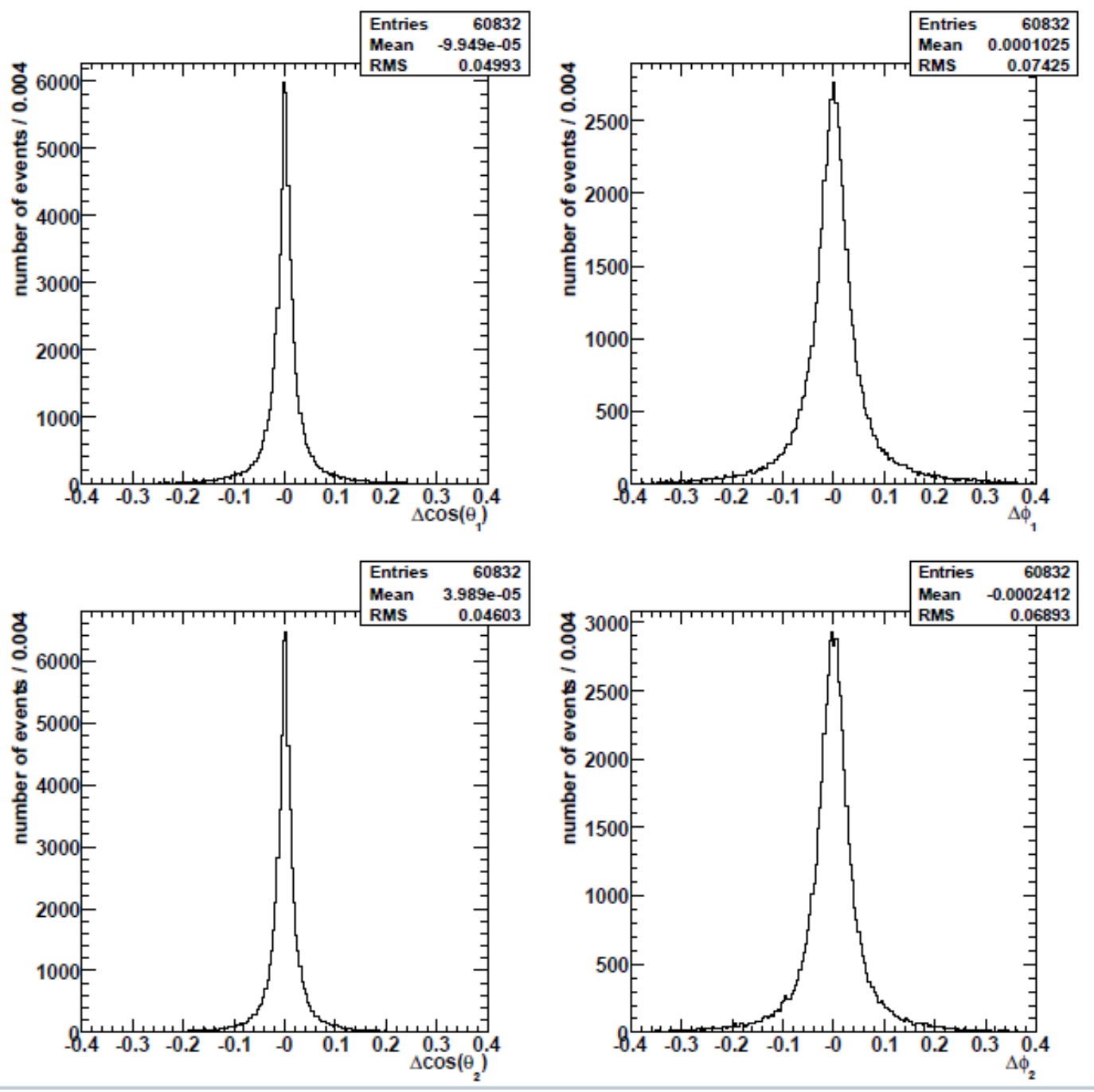

Figure 6.16: Angular resolution for the angles of the two light flavor quarks from the $W$ boson decay [93. The resolution is defined as the the difference between the quark angle and the measured jet angle. Note that the $\theta$ angle is shown in terms of $\cos \theta$ to match the natural spherical integrand over the solid angle, $d \cos \theta d \phi$. All four distributions are centered at 0 and show now significant bias. 


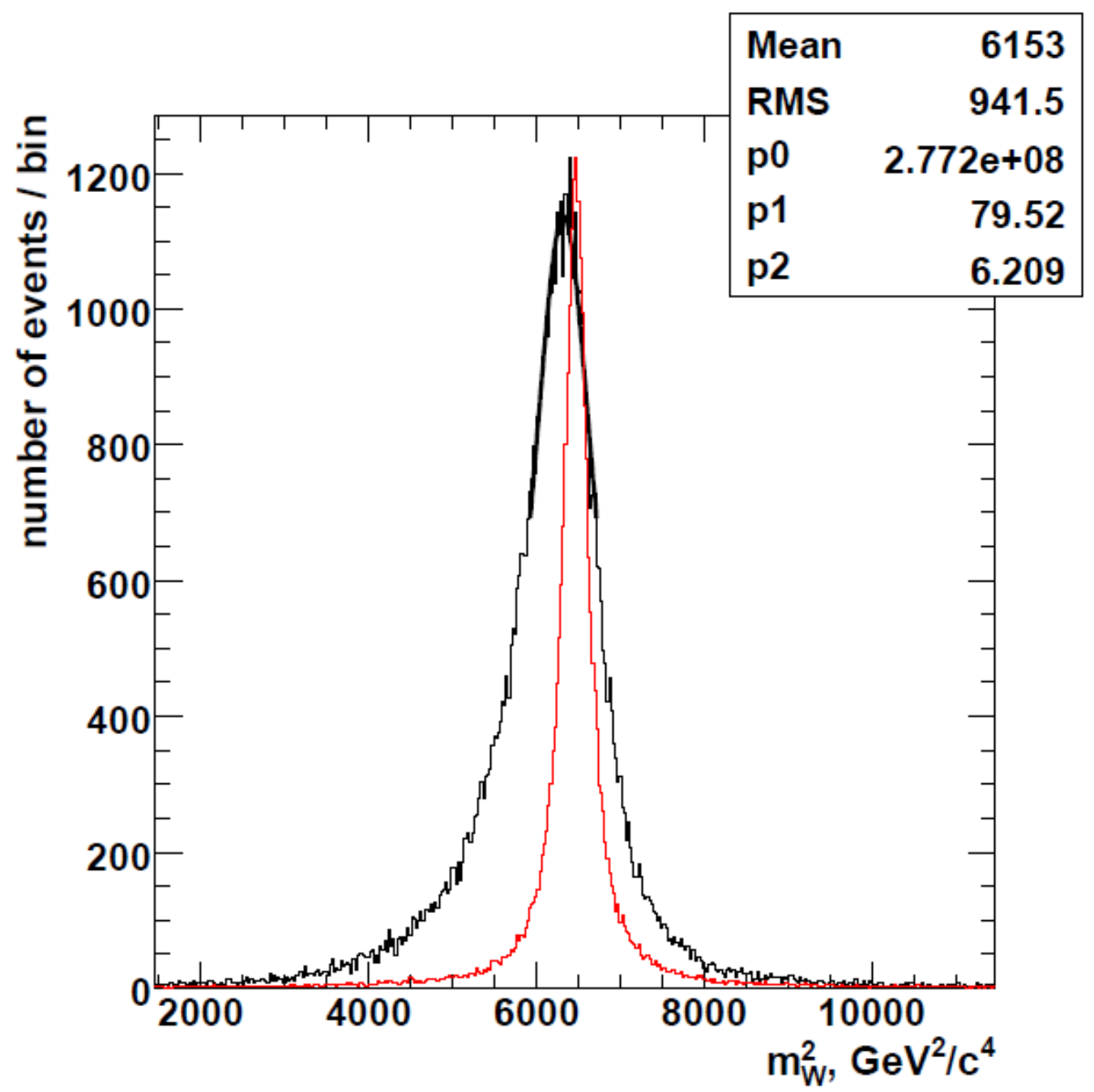

Figure 6.17: Distribution of $m_{W}^{2}$ as determined by the 4-vectors of the two decay daughters [93]. For both distributions, the decay daughter particles' energy is taken from the quark information in the MC. However, the angles of the decay daughters are taken from the quarks to build the red distribution and the jets to build the black distribution. Using the measured angles from the reconstructed jets not only lowers the peak of the distribution, but it also introduces a significant bias towards lower $m_{W}^{2}$. 


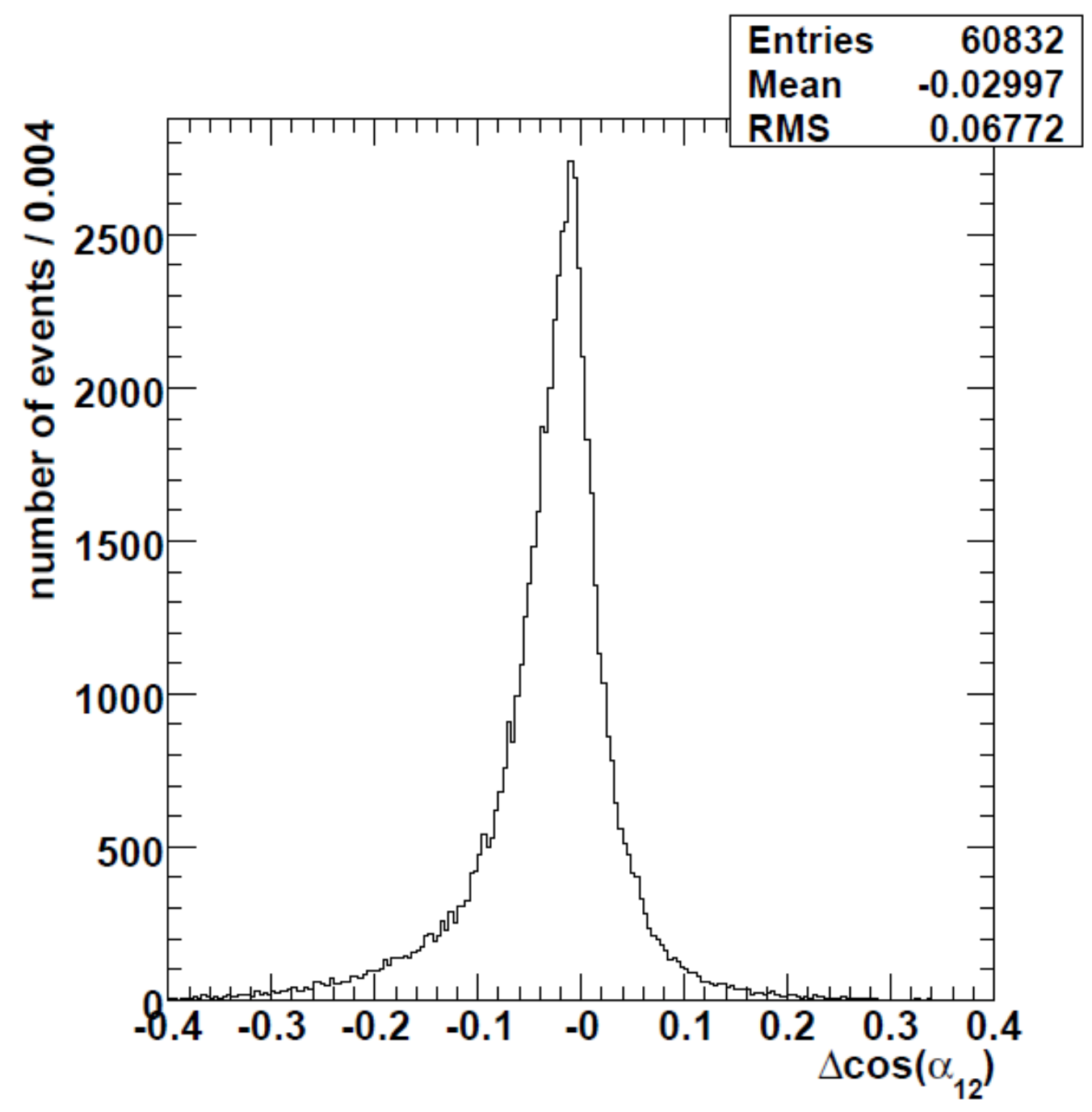

Figure 6.18: Distribution of $\Delta \cos \alpha_{12}$ defined as $\cos \alpha_{12}^{y}-\cos \alpha_{12}^{x}$ where $\alpha_{12}$ is the angle between the two daughters from the $W$ boson decay [93]. We see that there is a bias toward the reconstructed jets being closer together than the true quarks. This effect is not observable in the resolution of the angles by themselves as it is washed out by the spherical symmetry of the $W$ boson decay. This bias explains the effect on $m_{W}^{2}$ observed in Fig. 6.17. 
means we are able to fully describe the angles from the two jets without introducing any extra variables. In this new coordinate system, we find the skewness only exists in the angle between the jets. The other 3 variables have narrow resolutions centered at 0 , so we choose to treat them as delta functions in the signal probability calculation.

The angular $\mathrm{TF}$ for the angle between the two jets from the $W$ decay is fit to the distribution of $\Delta \cos \alpha_{12} \equiv \cos \alpha_{12}^{y}-\cos \alpha_{12}^{x}$. We chose a skew-Cauchy distribution summed with two Gaussian functions to fit a distribution with 10 fit parameters $p_{i}\left(p_{0}\right.$ through $p_{9}$ ) with the form:

$$
W_{A}^{12}\left(\Delta \cos \alpha_{12}\right)=\frac{1}{p_{0}+p_{4}+p_{7}}\left(p_{0} \frac{1+\frac{2}{\pi} \arctan \left(p_{2} p_{3} X_{1}\right)}{\pi p_{2}\left(1+X_{1}^{2}\right)}+p_{4} \frac{e^{-\frac{1}{2} X_{2}^{2}}}{\sqrt{2 \pi} p_{6}}+p_{7} \frac{e^{-\frac{1}{2} X_{3}^{2}}}{\sqrt{2 \pi} p_{9}}\right)
$$

where:

$$
\begin{aligned}
& X_{1}=\frac{\Delta \cos \alpha_{12}-p_{1}}{p_{2}} \\
& X_{2}=\frac{\Delta \cos \alpha_{12}-p_{5}}{p_{6}} \\
& X_{2}=\frac{\Delta \cos \alpha_{12}-p_{8}}{p_{9}} .
\end{aligned}
$$

As shown in Fig. 6.19, the bias in $\alpha_{12}$ is very small when the two jets are back to back and grows as the $\cos \alpha_{12}$ increases which again points to the effect being the result of color interactions between the quarks. As a result, we separate the angular TF into 12 bins in $\cos \alpha_{12}$. The first 10 bins are 0.2 steps from a $\cos \alpha_{12}$ of -1.0 to 1.0. The final two bins are used for cases where the jets are produced nearly collinearly with the $W$ boson. When the jets are nearly collinear with the $W$ boson, they are close enough together that any large skewness that would cause them to reconstruct closer together may lead to the jets failing to reconstruct as separate jets. These events would then fail to pass the selection requirements which leads to us not observing the skewness in this case. The distribution of $\Delta \cos \alpha_{12}$ and the angular TF for each bin is shown in Fig. 6.19. The different widths for the distribution across the 12 bins is actually just a result of the definition of $\cos \alpha_{12}$. Changes in $\Delta \cos \theta_{12}$ represent a larger effect when $\cos \alpha_{12}$ 

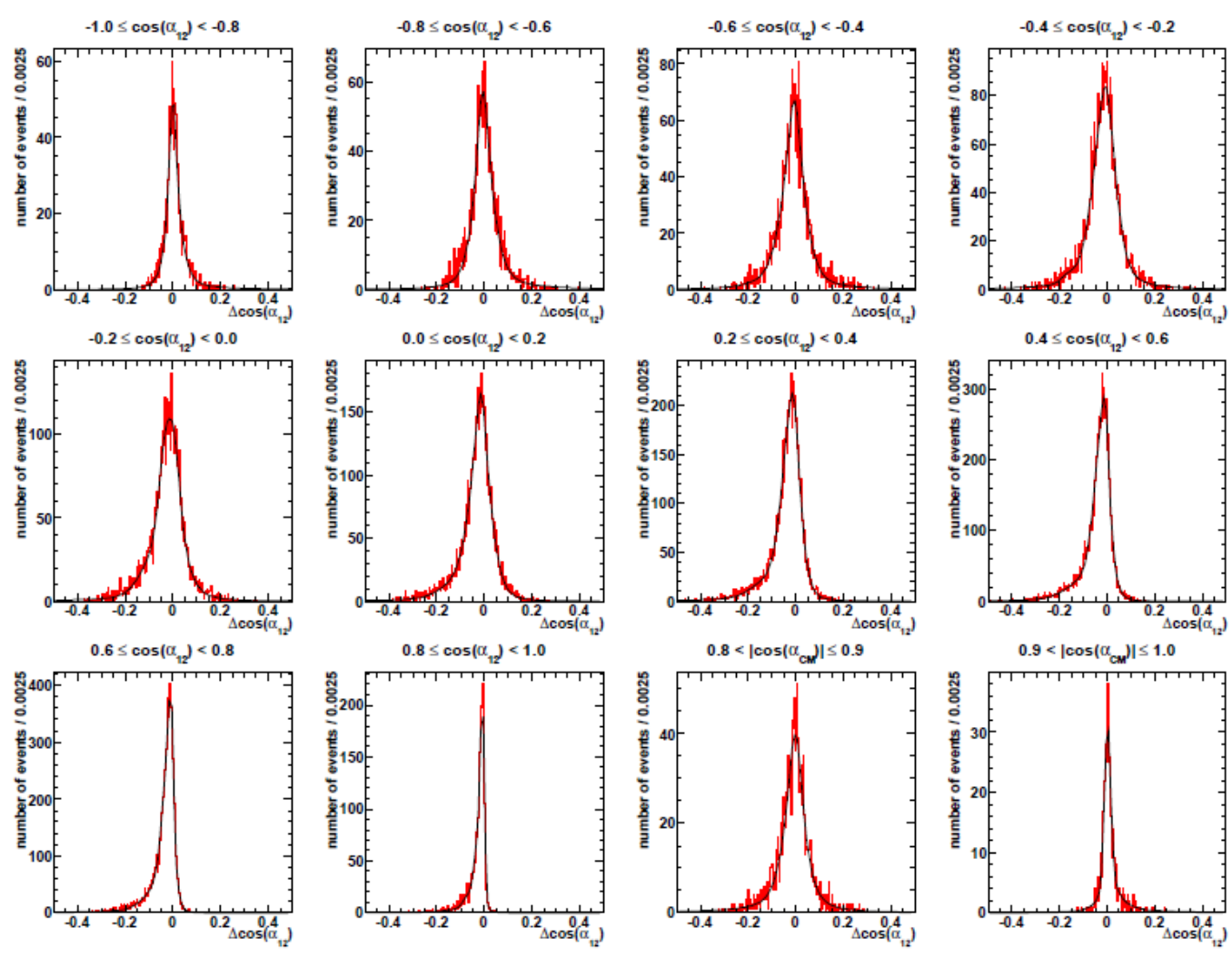

Figure 6.19: Distribution of $\Delta \cos \alpha_{12}$ shown for all 12 bins in which the angular TF component is fit. The red line shows the distribution from MC while the black line shows the resulting TF fit 93 .

is large than when it is closer to 0 . In each of the 12 bins, we fit the $p_{i}$ parameters with a Minuit 95] fitter just as we did with the energy TFs.

Finally, we test the results of this piece of the angular TF by convoluting the distribution of $\Delta \cos \theta_{12}$ with this light quark angular TF. The distribution before and after applying the angular TF is shown in Fig. 6.20. Although the light quark angular transfer function smears out the distribution of $\Delta \cos \theta_{12}$, we observe that the distribution no longer shows any skewness. Thus, by integrating over $\cos \theta_{12}$ in the signal probability and convoluting the probability with this TF, we remove the effect of the bias on the measurement.

A similar but smaller effect is seen in the angle between the $W$ boson and $b$ quark from the hadronic top quark decay (Fig. 6.21). In this case though, the angles of the $W$ 


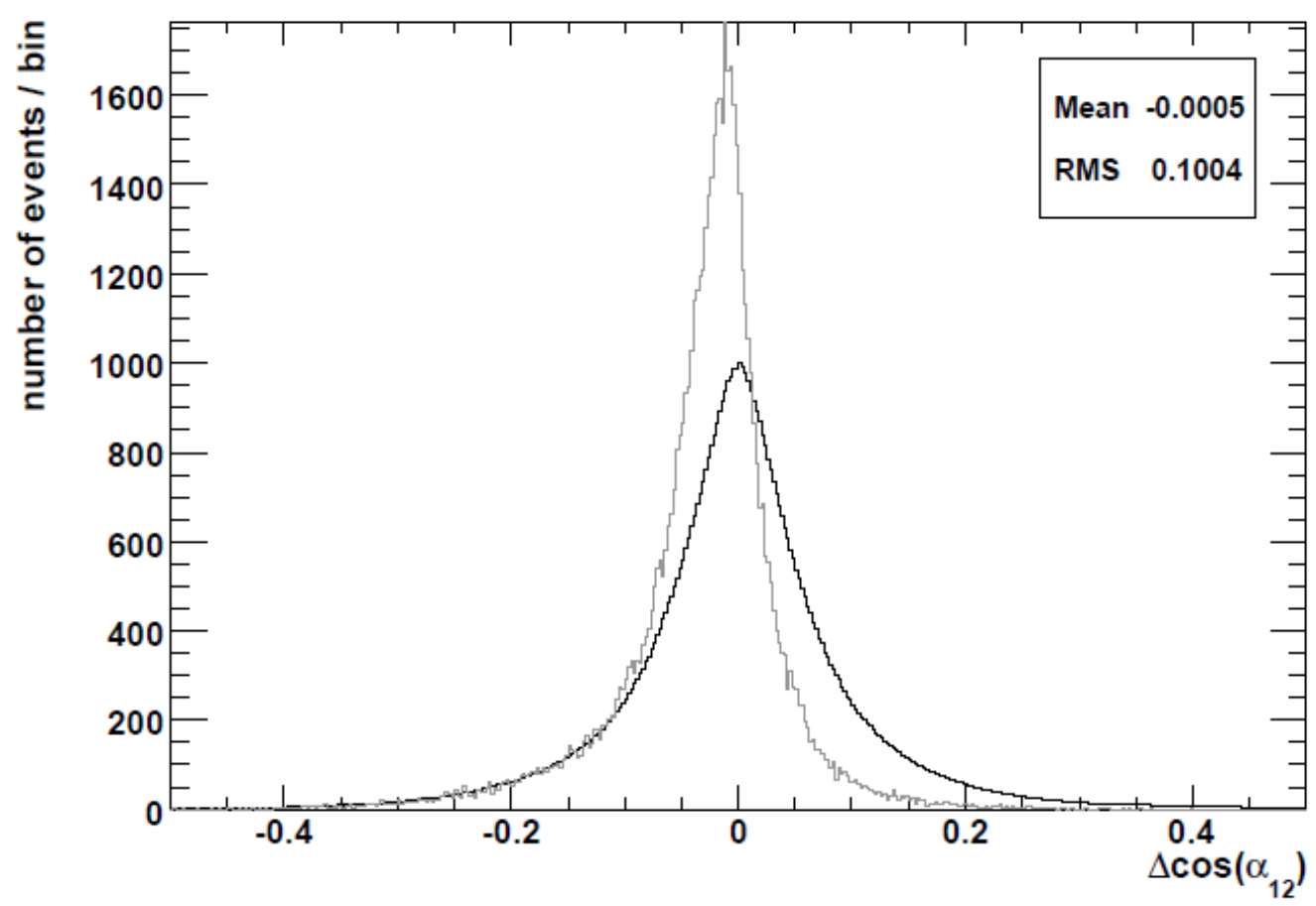

Figure 6.20: Distribution of $\Delta \cos \alpha_{12}$ [93]. The gray line shows the original skewed distribution before the angular TF component is applied. The black line shows the result after the original distribution is convoluted with the angular TF $\alpha_{12}$ component. Although the angular TF increases the width of the resolution, it removes any bias from the reconstructed angles. As a result, by convoluting the signal probability by the angular TF and integrating over the variables $\cos \alpha_{12}$, we can calculate the signal probability without the effects of the bias from the measured jet angles. 
appear correct while the $b$ quark shifts towards the $W$. We perform a similar change of variable with the only difference being that the constant value of $c_{W b}$ is now chosen to be the $\phi$ angle of the $W$ boson in the new coordinate frame. We parameterize an angular TF for the angle between the $W$ and $b$ quark with a skew-Cauchy distribution summed with a single Gaussian function to fit the distribution of $\Delta \cos \alpha_{W b} \equiv \cos \alpha_{W b}^{y}-\cos \alpha_{W b}^{x}$. This equation has seven parameters $q_{i}\left(q_{0}\right.$ through $\left.q_{6}\right)$ and takes the form:

$$
W_{A}^{W b}\left(\Delta \cos \alpha_{W b}\right)=\frac{1}{q_{0}+q_{4}}\left(q_{0} \frac{1+\frac{2}{\pi} \arctan \left(q_{2} q_{3} Y_{1}\right)}{\pi q_{2}\left(1+Y_{1}^{2}\right)}+q_{4} \frac{e^{-\frac{1}{2} Y_{2}^{2}}}{\sqrt{2 \pi} q_{6}}\right),
$$

where:

$$
\begin{gathered}
Y_{1}=\frac{\Delta \cos \alpha_{W b}-q_{1}}{q_{2}} \\
Y_{2}=\frac{\Delta \cos \alpha_{W b}-q_{5}}{q_{6}} .
\end{gathered}
$$

We use the same 12 bin setup as we did for the $\cos \alpha_{12}$ function. The distribution from MC events as well as the fit angular TF across all 12 bins are shown in Fig. 6.22 .

Putting this all together, the angular TF is the product of the two functions for $\Delta \cos \alpha_{12}$ and $\Delta \cos \alpha_{W B}$ given in Eqns. 6.42 and 6.46. We then integrate over the two angles $\cos \alpha_{12}$ and $\cos \alpha_{W b}$ in the signal probability calculation while all other parton angles necessary for the calculation of the event kinematics (as given in App. C) are assumed to be well measured by the corresponding jet angles. The resulting effects on the $W$ boson and top quark mass from using the angular TF are shown in Fig. 6.23

\subsection{Maximization and Fit of the Likelihood Function}

After calculating the negative log likelihood function as a function of $M_{\text {top }}$ as detailed in Sec. 6.2 we fit it with a $2^{\text {nd }}$ order polynomial to extract $M_{t o p}$ and its statistical uncertainty. The function is expected to be parabolic due to the Gaussian shape of the signal probability combined with the flat term with respect to $M_{\text {top }}$ from the background probability.

First, we use the Minuit function minimization algorithm [95] to maximize the likelihood function versus the signal fraction $c_{s}$ for each step in the range of considered 


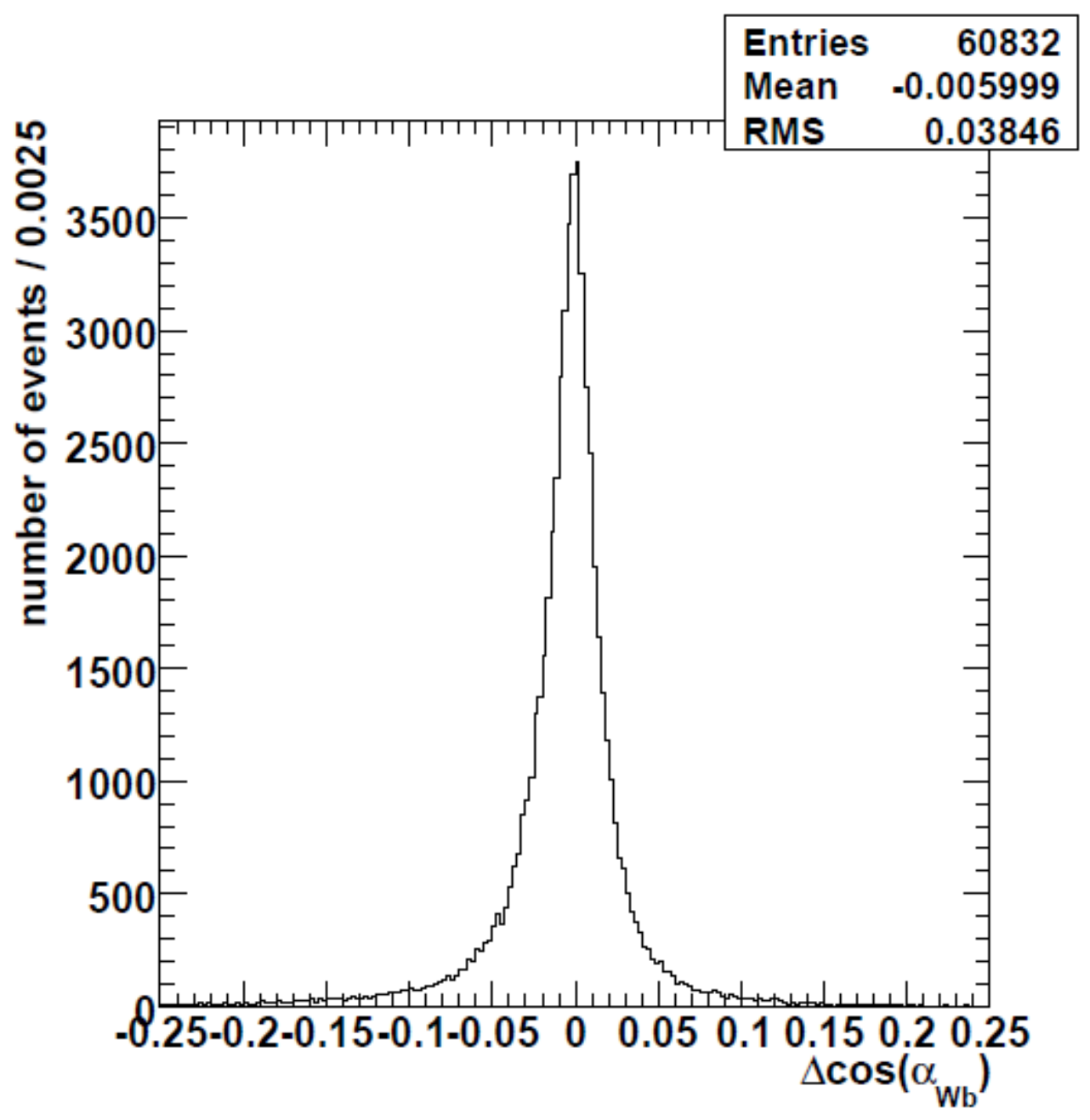

Figure 6.21: Distribution of $\Delta \cos \alpha_{W b}$ defined as $\cos \alpha_{W b}^{y}-\cos \alpha_{W b}^{x}$ where $\alpha_{W b}$ is the angle between the $W$ boson and the $b$ quark from the top quark decay [93]. Similarly to the result from the two light flavor quark jets seen in Fig. 6.18, we see a bias toward the reconstructed particles being closer together than the true particles. 

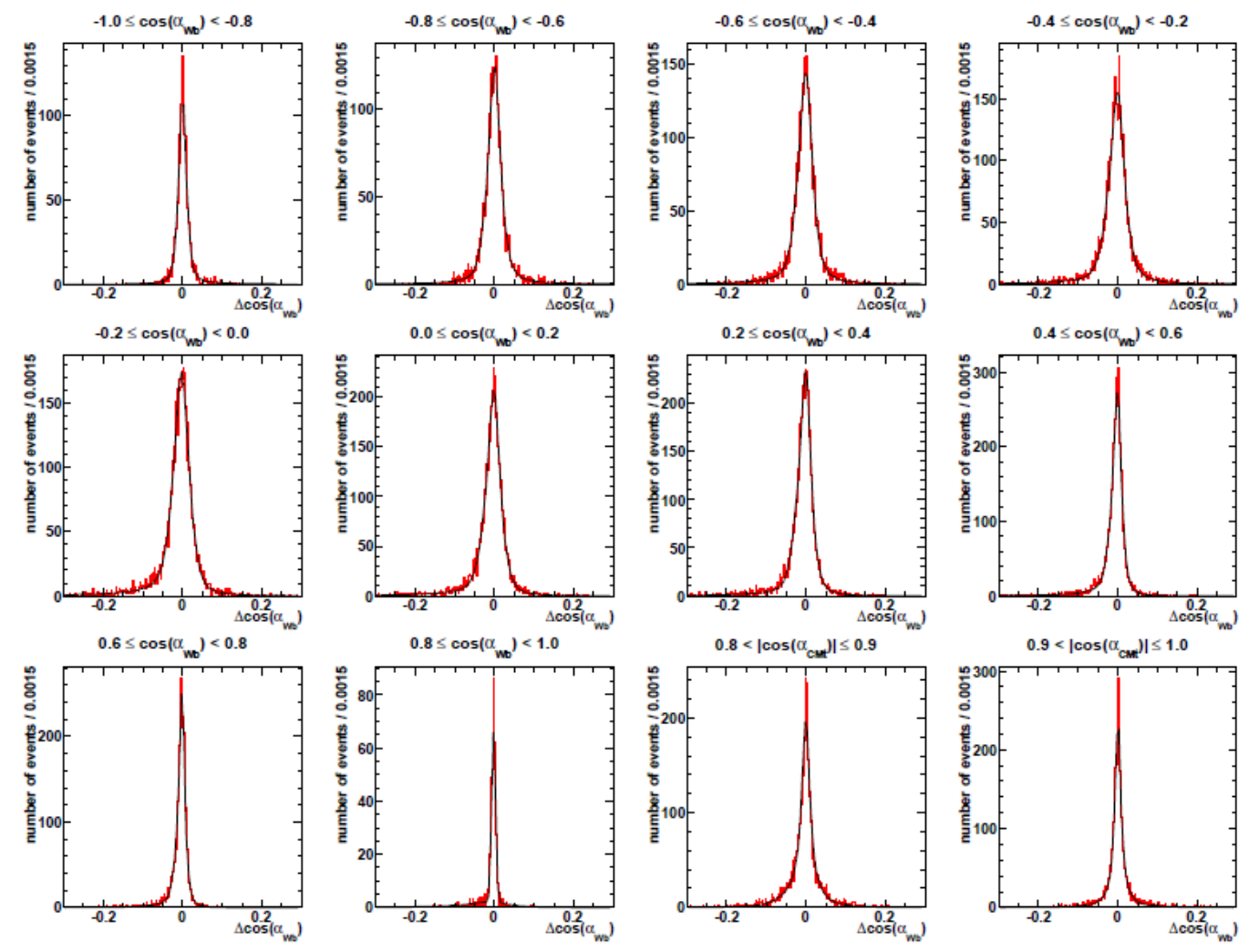

Figure 6.22: Distribution of $\Delta \cos \alpha_{W b}$ shown for all 12 bins in which the angular TF component is fit. The red line shows the distribution from MC while the black line shows the resulting TF fit [93]. 

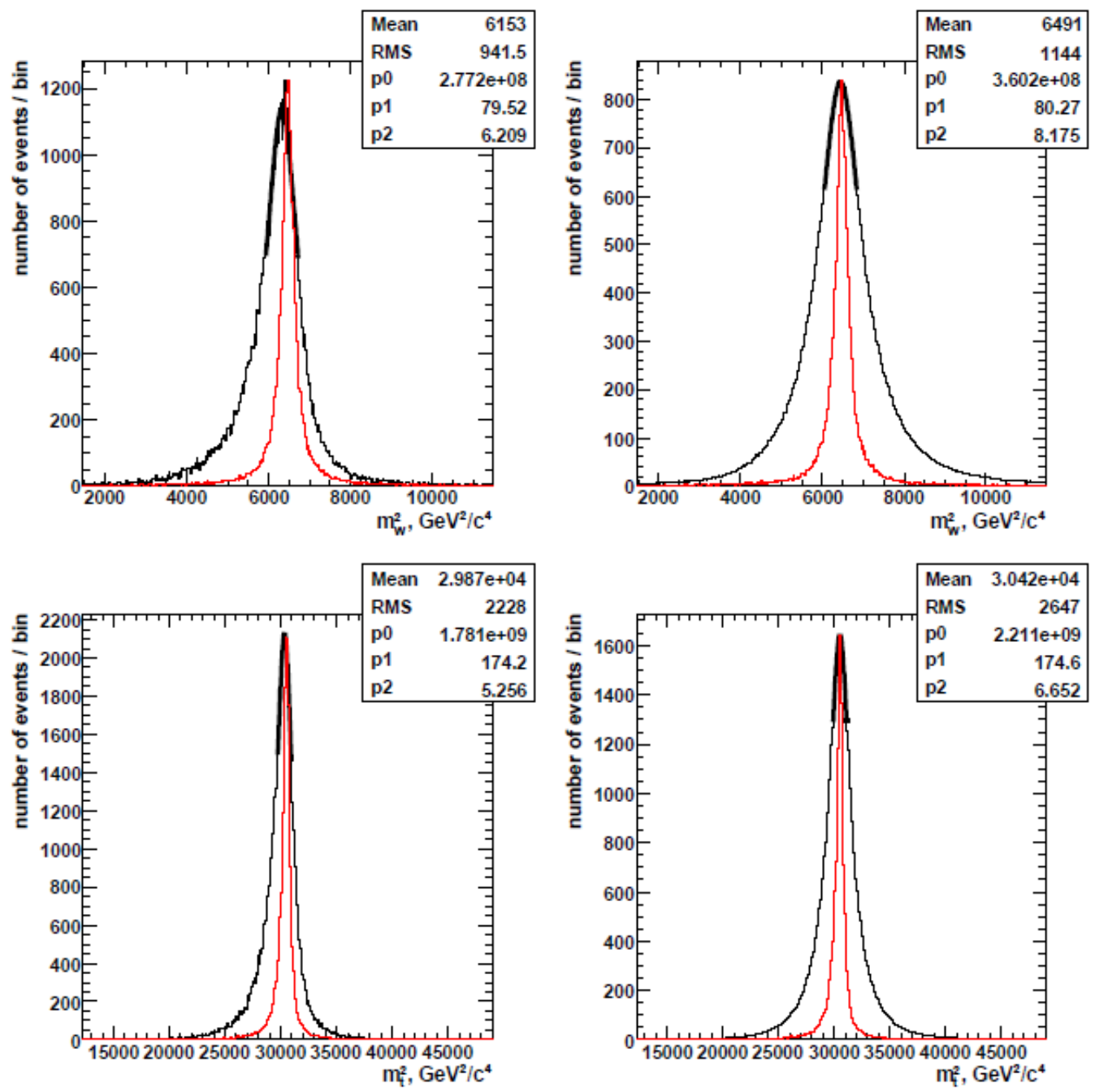

Figure 6.23: Final cross check of the angular TF [93]. The top row shows $m_{W}^{2}$ from the 4 -vectors of its two daughter particles. The bottom row shows $M_{\text {top }}^{2}$ as calculated from the 4 -vectors of the $W$ boson and $b$ quark. In all 4 distributions, the red distribution is made using the 4-vectors of the truth level objects from the MC. The black distribution is made using the energies of the truth level objects in the $\mathrm{MC}$ but the angles of the reconstructed objects. The left and right columns show the result before and after the measured angles are convoluted by the angular transfer function. For both the $W$ boson and the top quark, by comparing the left and right columns we can see that the application of the angular transfer both moves the peak of the distribution to the correct value and symmetrizes the distribution about this peak. 
top mass. The parameter $c_{s}$ is allowed to vary between 0 and 1 , but it is highly restricted by the Gaussian constraint on $c_{s}$ in the likelihood function.

Once the likelihood is maximized for $c_{s}$, we produce the distribution of the negative $\log$ likelihood as a function of top quark mass. We use a $\chi^{2}$ regression to fit this distribution with a $2^{\text {nd }}$ order polynomial. The fitter begins with a $12 \mathrm{GeV}$ window centered around the minimum bin. If the $12 \mathrm{GeV}$ window exceeds the edge of the considered distribution, we restrict the fit window to the edge of the considered distribution. We extract the central value of $M_{t o p}$ from the fit by finding the point at which the derivative of the fit is equal to 0 . A $1 \sigma$ statistical uncertainty on $M_{t o p}$ is derived as the distance in $M_{\text {top }}$ from the central value at which the negative log likelihood function increases by $\frac{1}{2}$. For a $2^{\text {nd }}$ order polynomial of form $A x^{2}+B x+C=0$, this gives an $M_{\text {top }}$ measurement of $-\frac{B}{2 A} \pm \frac{1}{\sqrt{2 A}}$ (The derivation of this is given in App. A.).

With the mean and standard deviation calculated from the original fit, we then refit with a new window to remove bias from the arbitrarily chosen fit window. We resize the fit window to the mean \pm 2 times the standard deviation calculated from the fit. Since approximately $95 \%$ of the population of a Gaussian is within $2 \sigma$ of the mean, this method should ensure that we fit over a range the includes the majority of the information while avoiding any edge effects from fitting too far out into the tails of the distribution. We iteratively loop over this $2 \sigma$ fit window three times which we have found is enough iterations to stablize fit.

\subsection{Calibration}

Before we measure $M_{t o p}$ in the data, we check that the method is properly calibrated. We use the $21 \mathrm{MC}$ signal samples with different masses to do this. We measure $M_{\text {top }}$ in each sample and compare it to the known $M_{t o p}$ for that sample. Since the samples are not correlated, we also consider possible statistical fluctuations in the top quark mass measurement between samples. To measure $M_{t o p}$ for each sample, we generate an ensemble of PE's from each signal MC with fully simulated backgrounds. We choose to select PE's with 41 events to match the data. For each mass sample, we run multiple 
PE's and fit a Gaussian function to the resulting distribution of measured $M_{t o p}$. As we increase the number of PE's, the Gaussian becomes smoother, and we can better measure the mean $M_{t o p}$. However, we are limited by the total number of events from which we can select to build independent PE's. We could build more PE's either by generating more $\mathrm{MC}$ events, but this is a time consuming process to generate enough MC to get a significant addition to the number of events passing the selection and then running these events through calculations of the signal and background probabilities. The other way to generate more PE's is to resample the MC events. As we build PE's, we resample events with replacement from the MC events. We limit the amount of resampling to the square of the number of independent PE's we can generate from the original MC events without resampling. Above this threshold, there is a risk that many of the PE's may be filled with the same resampled event multiple times. As long we stay below this threshold, we resample events to build more PE's without altering the mean of the measured Gaussian distribution as resampling the events provides no extra information. This allows us to develop a smooth Gaussian distribution that can be well fit. We fit this distribution with a Gaussian function using a $\chi^{2}$ fitter to derive the mean of the top quark mass measurement for each sample.

Since the different mass samples are uncorrelated, we consider the possible spread of the mass measurement from statistical fluctuations so that we do not confuse a statistical fluctuation for a bug in the measurement. Unfortunately, due to the use of resampling, we cannot simply measure the RMS of the resulting Gaussian distribution to estimate the statistical uncertainty on the mean. Although resampling provides no extra information in the calculation of the mean, it does artificially shrink the width of the distribution as it simulates having more independent events than we really do. As a result, we use the bootstrapping method [96] to estimate the uncertainty on the mean of the distribution. This method is detailed in App. F.

With the mean and uncertainty of the top quark mass measured from each of the $21 \mathrm{MC}$ samples, we check the distribution of output $M_{\text {top }}$ versus input $M_{\text {top }}$. We fit this with a $1^{\text {st }}$ order polynomial using a $\chi^{2}$ regression. Ideally, we expect to find a 1:1 relationship between the measured and input mass which would give a line with a slope 
of 1 and an y-intercept of 0 . However, we find in Fig. 6.24 that the fit line does not meet these requirements. From the fit, we derive the following calibration function for the top quark mass:

$$
M_{\text {top }}^{\text {corr }}=\frac{\left(M_{\text {top }}^{\text {meas }}-30.94\right)}{0.806} .
$$

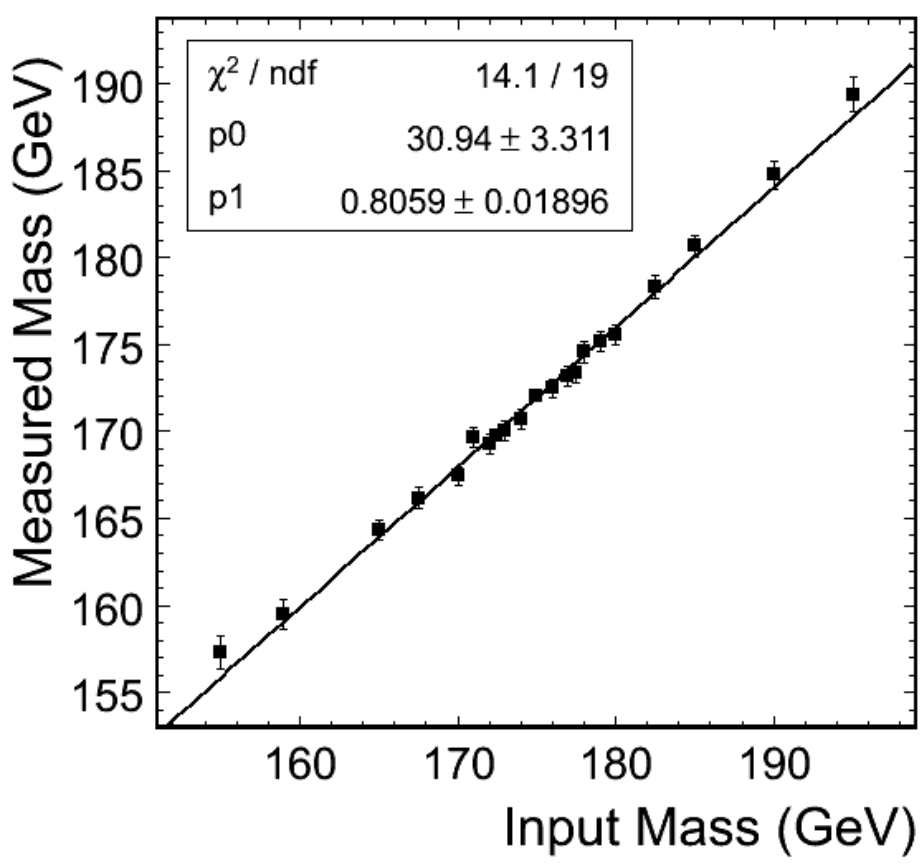

Figure 6.24: Output fitted $M_{t o p}$ versus input $M_{t o p}$ in $\tau+$ jets events. The fit is used to derive the initial calibration function in Eqn. 6.49.

We apply this calibration function to the resulting measurement from each PE and again check the output fitted $M_{\text {top }}$ against the input $M_{t o p}$ in Fig. 6.25 to make sure the calibration function behaved as intended. We now find the expected result with a slope of 1 and an y-intercept of 0 within the uncertainties of the fit.

Finally, we check the "pull" distributions for each sample to verify that there is no bias in the measurement and that we are correctly evaluating the uncertainty. The pull is defined as:

$$
\text { Pull }=\frac{M_{\text {top }}^{\text {measured }}-M_{\text {top }}^{\text {truth }}}{\sigma_{M}^{\text {measured }}},
$$

where $M_{\text {top }}^{\text {measured }}$ is the measured top quark mass from a PE, $M_{\text {top }}^{\text {truth }}$ is the true top 


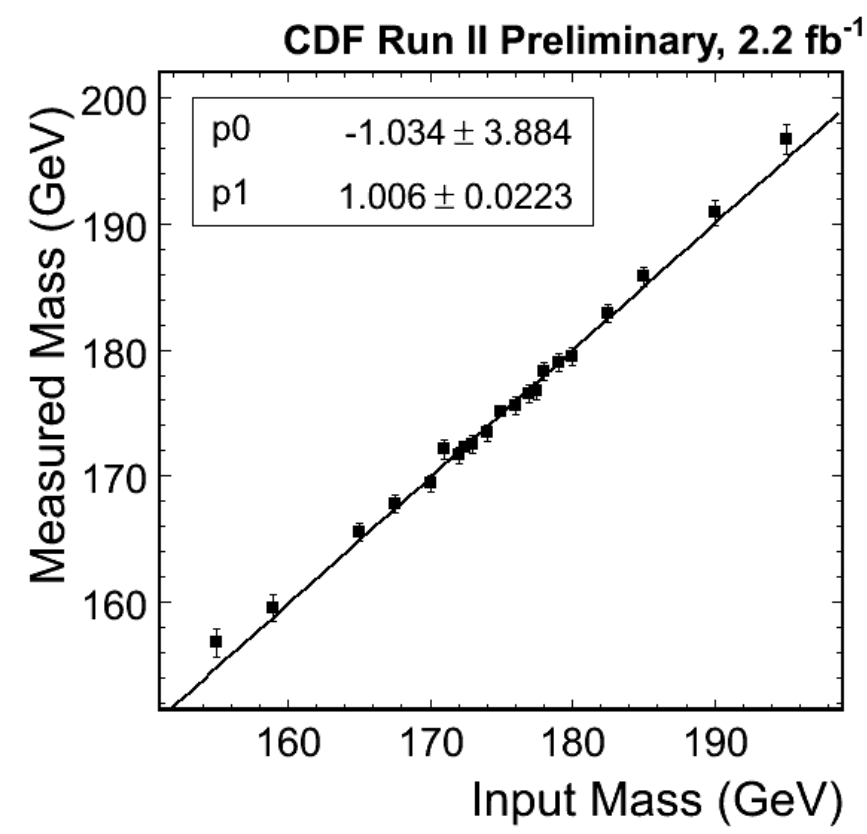

Figure 6.25: Output fitted $M_{t o p}$ versus input $M_{t o p}$ in $\tau+$ jets events after the initial calibration is applied. We now find no bias in the measurement.

quark mass in the signal $\mathrm{MC}$, and $\sigma_{M}^{\text {measured }}$ is the uncertainty on the measured top quark mass from a single PE. If the measurement is properly calibrated and the uncertainty is being correctly estimated, the distribution of pulls from the PE's in an ensemble for a given input $M_{t o p}$ should be Gaussian with a mean of 0 and a width of 1 .

Due to the previous calibration function, we already know that the measurement is unbiased, so first, we check the distribution of pull widths versus the input $M_{t o p}$ in Fig. 6.26. We find that the uncertainty is being underestimated by a factor of nearly 1.76 which has no apparent dependence on $M_{t o p}$, so, to correct for this, we apply the following calibration function to the uncertainty from each PE:

$$
\delta M_{\text {top }}^{\text {corr }}=(1.76) \times \delta M_{\text {top }}
$$

The size of this correction is partly due to the method used to calibrate the top quark mass. Because the initial calibration function (Eqn. 6.49) has a dependence on $M_{t o p}$, the function shifts higher values of $M_{t o p}$ more than it shifts lower values of $M_{t o p}$. This essentially stretches the x-axis of the original likelihood function. If we 
were to apply the calibration across the entire likelihood function, we would stretch the function along the $\mathrm{x}$-axis and measure a greater uncertainty than what we measure from the uncalibrated likelihood function. Rather than applying the calibration across the entire likelihood function, we choose to apply it to the measured top quark mass. This means that we do not account for the stretching of the likelihood function when we measure the uncertainty on the top quark mass, and we expect to underestimate its value as a result. From the initial calibration function (Eqn. 6.49), we estimate that the calibration should increase the uncertainty by a factor of $\frac{1}{0.806}=1.24$. By dividing 1.76 by this value, we find that the correction needed for the uncertainty would be 1.42 if we had applied the calibration across the entire likelihood function.

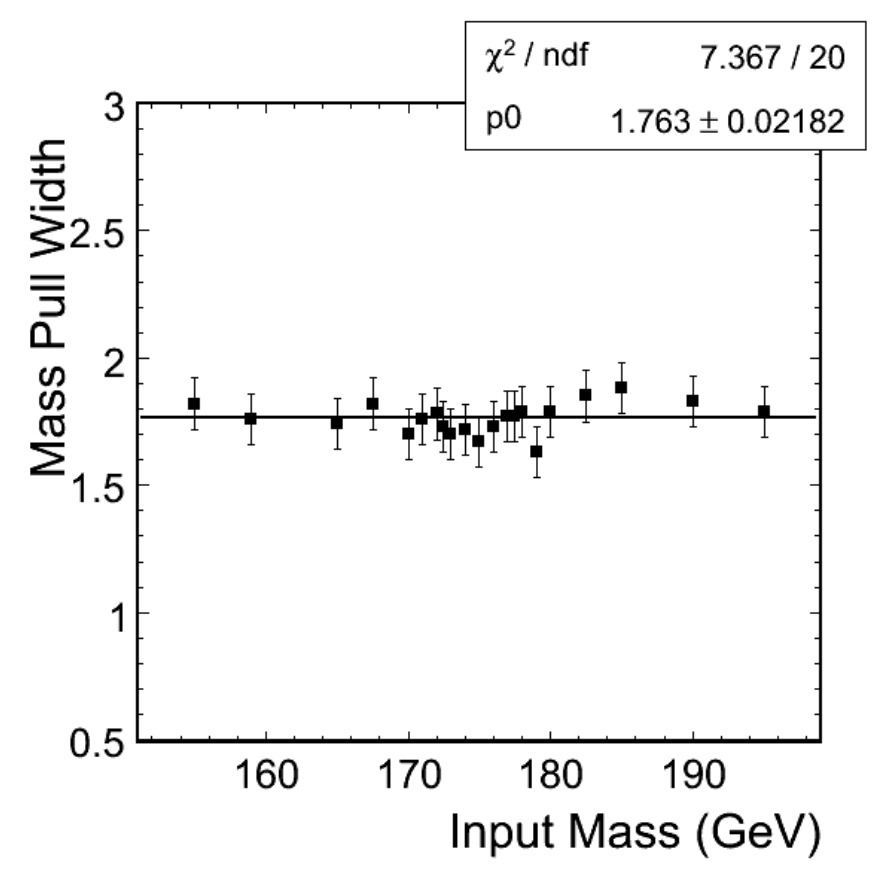

Figure 6.26: Mass measurement pull width versus input $M_{\text {top }}$ for $\tau+$ jets events after the initial calibration function is applied. We find that the uncertainty on the measurement of $M_{t o p}$ is underestimated by a factor of 1.76 .

Now that we have calibrated the $M_{t o p}$ measurement and its uncertainty, we again check to make sure that the analysis is unbiased. Figure 6.27 shows the residual (defined as the measured $M_{t o p}$ minus the input $M_{t o p}$ ), pull, and pull width as a function of input $M_{\text {top }}$. We can see from this figure that the measurement and its uncertainty is now 
unbiased.

\subsection{Expected Uncertainty}

With a fully calibrated method, the final thing to do before we make the measurement in the data is to estimate the statistical uncertainty we expect to measure. We check the distribution of the uncertainty on the measured $M_{t o p}$ from each of the 21 signal MC samples. Since the relative uncertainty is expected to be fairly stable across $M_{t o p}$, we find the expected uncertainty has a linear dependence on $M_{t o p}$. To predict the uncertainty for any measured value of $M_{t o p}$, we fit this distribution with a $1^{\text {st }}$ order polynomial in Fig. 6.28. For the nominal signal point with mass $172.5 \mathrm{GeV}$, we expect a statistical uncertainty of $6.0 \mathrm{GeV}$ for the measurement.

\subsection{Measurement of $M_{t o p}$}

We now measure the top quark mass in the data by forming the likelihood function from the 41 events 2 The negative log likelihood function is shown in Fig. 6.29. This likelihood distribution has not yet had the calibration functions detailed in Sec. 6.6 applied. We fit the likelihood function and apply the calibration functions to derive the top quark mass measurement. After doing so, we measure the top quark mass to be $172.7 \pm 9.3$ (stat.) GeV. Since the measured statistical uncertainty is higher than the expected uncertainty of $6.0 \mathrm{GeV}$, we check the measured uncertainty against the uncertainty from an ensemble of PE's with a top quark mass of $172.5 \mathrm{GeV}$ in Fig. 6.30. We find that $4 \%$ of PE's have a higher uncertainty than the value we measure. The systematic uncertainties on the measurement of $M_{\text {top }}$ are detailed in Chap. 7.

\footnotetext{
${ }^{2}$ We lost two events due to the quadratic solver being unable to find an appropriate solution to the top quark decay kinematics. We seem to have possibly gotten unlucky here as this tends to happen with 1 in every 100 selected MC $t \bar{t}$ events.
} 

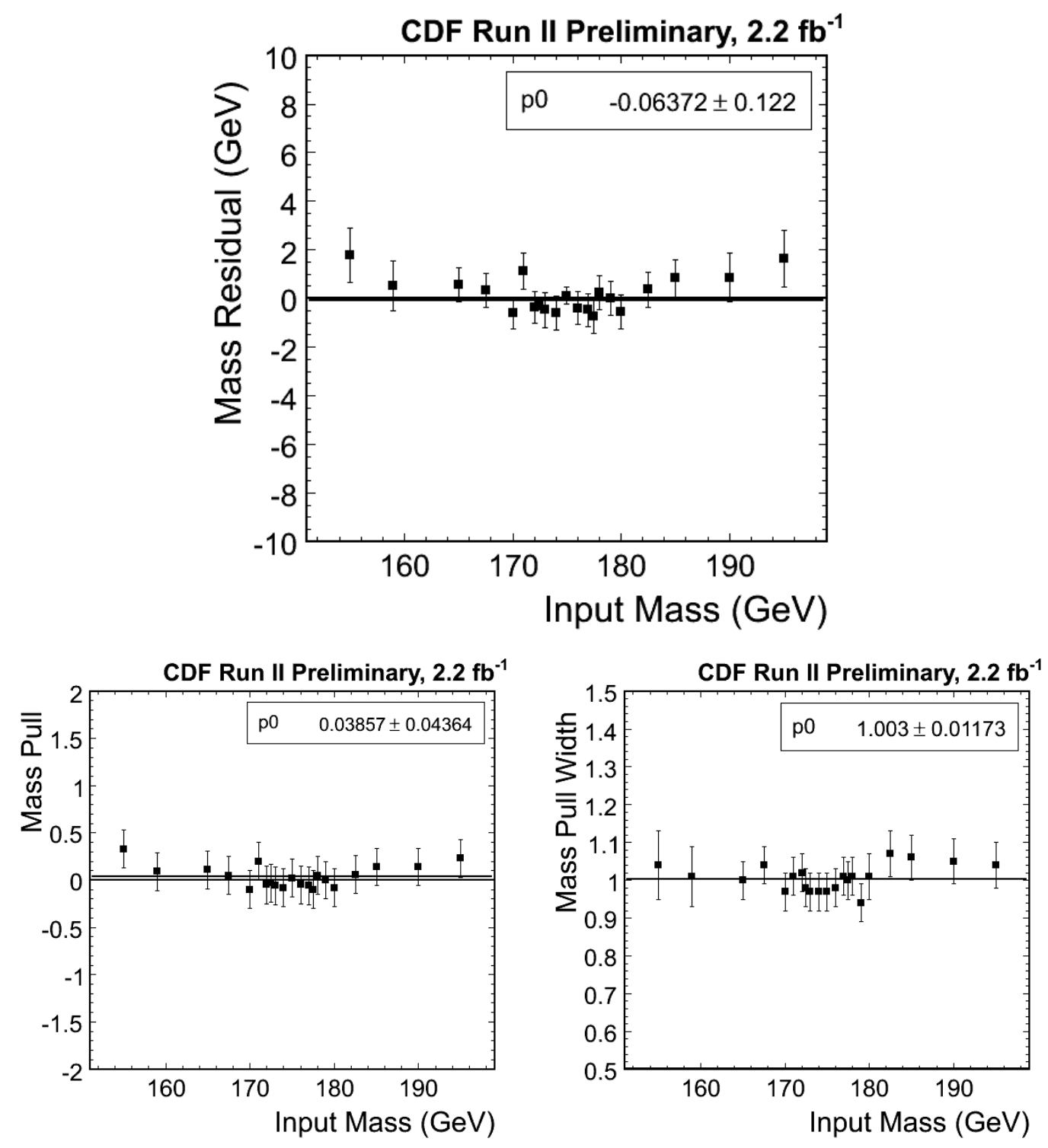

Figure 6.27: Residual mass (top), mass pull mean (bottom left) and mass pull width (bottom right) versus input $M_{\text {top }}$ for $\tau+$ jets events after all calibrations are applied. We now find that the $M_{\text {top }}$ measurement and its uncertainty are unbiased. 


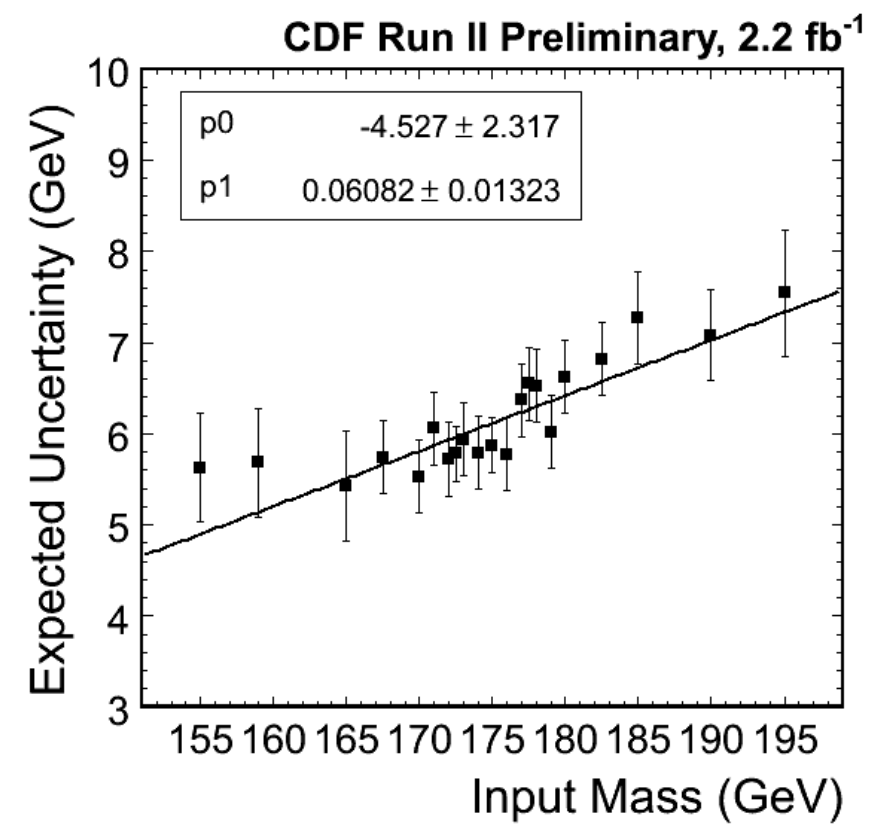

Figure 6.28: Expected statistical uncertainty on the top quark mass measured in $\tau+$ jets events as a function of $M_{t o p}$. For the nominal point of $M_{t o p}=172.5 \mathrm{GeV}$, we expect a statistical uncertainty of $6.0 \mathrm{GeV}$. 


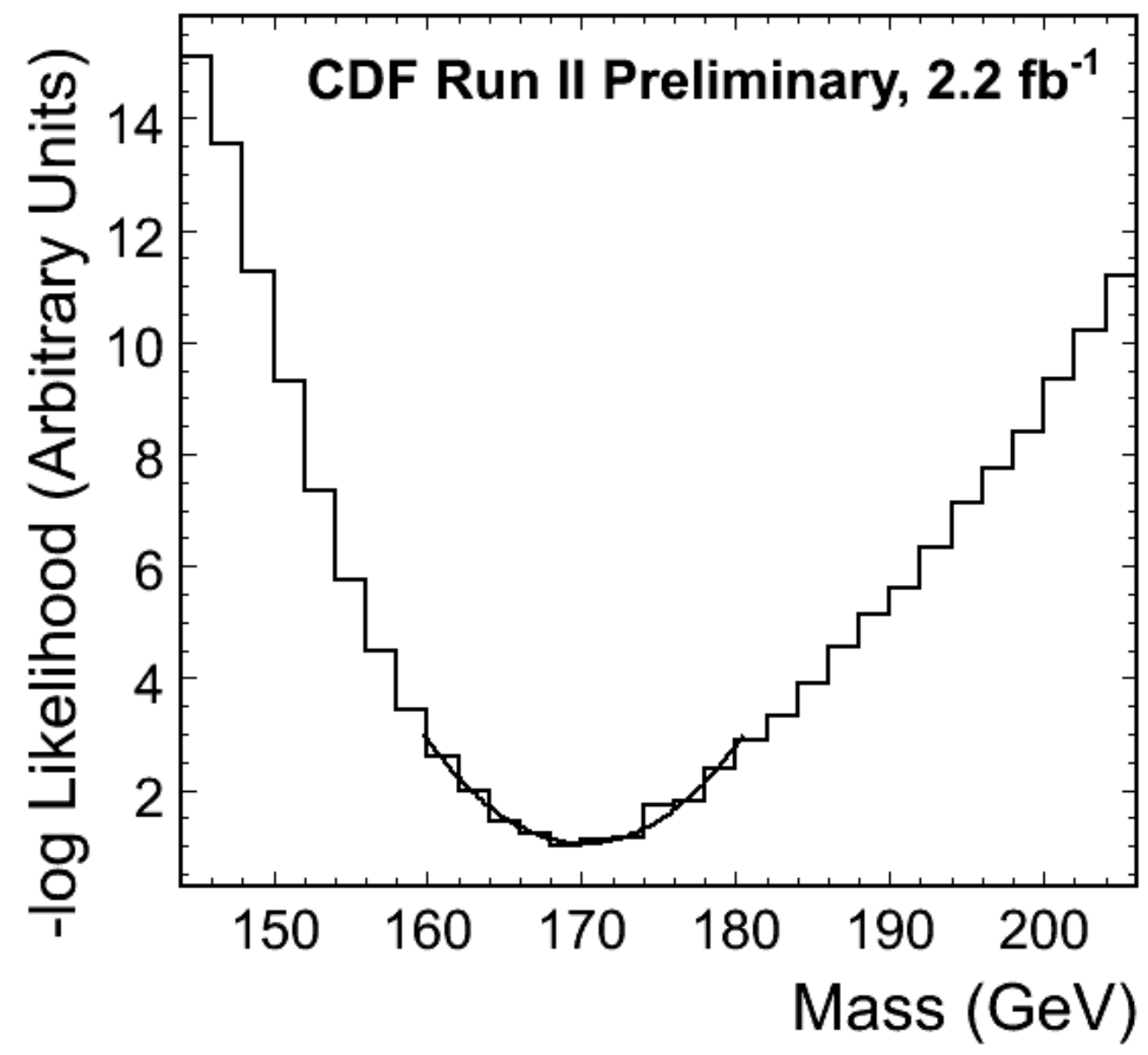

Figure 6.29: Negative log likelihood for all data events before calibration functions have been applied. The fit is shown on top of the likelihood. 


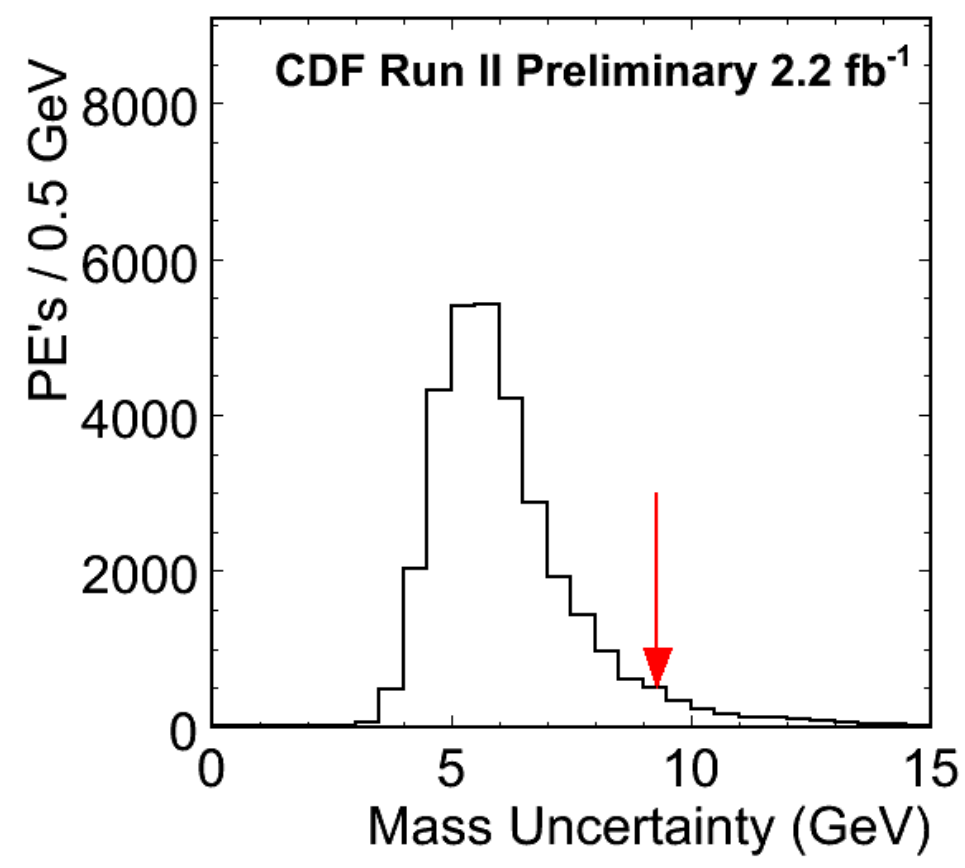

Figure 6.30: Expected uncertainty from an ensemble of PE's using $t \bar{t} \mathrm{MC}$ with the top quark mass equal to $172.5 \mathrm{GeV}$. The red line marks the $9.3 \mathrm{GeV}$ value we measured in the data. We find that approximately $4 \%$ of PE's give a statistical uncertainty greater than the value we measured. 


\section{Chapter 7 Systematic Uncertainties for $M_{t o p}$}

\section{$7.1 \quad$ Introduction}

This chapter describes the evaluation of the systematic uncertainties for the top quark mass measurement using hadronic $\tau+$ jets events. It includes a description of how each uncertainty is determined and additional MC samples for the measurement. Some of the methods and samples used to measure these uncertainties have already been described previously in Sec. 5.4. The systematic uncertainties considered for the $M_{t o p}$ measurement are the jet energy scale, MC generator, initial and final state radiation (I/FSR), color reconnection, background fraction, MC statistics, parton distribution function (PDF), gg fusion fraction, b-jet energy scale, lepton $P_{T}$, pileup, and calibration uncertainties.

\subsection{Overview}

For each systematic source, the uncertainty is evaluated by running an ensemble of pseudo-experiments (PE's) where the $t \bar{t} \mathrm{MC}$ has been altered to account for the changes due to the systematic source. If the systematic source is represented by a $\pm 1 \sigma$ shift, equivalent to a $68 \%$ confidence limit assuming Guassian errors, the uncertainty is then taken to be half the difference between the $1 \sigma$ shifted measurements if the shifts occur in opposite directions from the nominal value. If both shifted measurements move in the same direction from the nominal, the uncertainty is taken to be half of the largest difference from the nominal value. For systematic sources due to differences between two different models (such as the MC generator and color reconnection uncertainties), the uncertainty is taken to be the difference between the measured result using each 
model.

For systematic sources which are modeled by reweighting the $\mathrm{MC}$ events or changing the input parameters of the PE's (specifically, the PDF, gg fusion fraction, b-jet energy scale, background fraction, and pileup uncertainties), the $M_{\text {top }}$ measurements use the same or very similar sets of events. This means that the resulting measurements are highly correlated. Therefore, we ignore the statistical uncertainties on these measurements, and any differences between them can be assumed to be due to the systematic source. For some systematic sources (specifically the JES, MC generator, I/FSR, color reconnection, and lepton identification $\mathrm{SF}$ ) the uncertainty is measured using different MC samples produced by varying the systematic source. For these measurements, we run a single large $\mathrm{PE}$ with all signal $\mathrm{MC}$ events included and record the uncertainty on the measurement. When calculating the systematic uncertainty due to these sources, the uncertainty on the $M_{\text {top }}$ measurements is also considered because statistical fluctuations in the measurements could lead to underestimating the actual uncertainty. If the uncertainty on the different measurements is greater than the difference itself, we take uncertainty on the difference as the systematic uncertainty rather than the difference.

The following sections describe each systematic source and how the uncertainty is evaluated. The total systematic on the $M_{t o p}$ measurement and the contribution from each source is summarized in Tab. 7.1.

\subsection{Jet Energy Scale}

As described in Sec. 3.2.2, a series of energy corrections are applied to each jet to correct their energy for gaps and inefficiencies in the CDF calorimeters and the jet reconstruction algorithm. Each level of correction has an associated $1 \sigma$ uncertainty which we use to assign a systematic uncertainty on the $M_{t o p}$ measurement similarly to the method used for the $\sigma_{t \bar{t}}$ measurement described in Sec. 5.4.1.

To measure the effect of the JES systematic on $M_{t o p}$, we shift the energy of each jet in the signal $t \bar{t} \mathrm{MC}$ up or down by each $1 \sigma$ uncertainty. This is done separately for each of the 6 individual jet corrections. We rerun the event selection for each of the shifts 


\begin{tabular}{|l||r|}
\hline Source & Uncertainty $(\mathrm{GeV})$ \\
\hline JES & 3.37 \\
MC Generator & 0.50 \\
ISR/FSR & 0.34 \\
Color Reconnection & 0.50 \\
Background Fraction & 0.47 \\
MC Statistics & 0.14 \\
PDF & 0.12 \\
gg fusion & 0.17 \\
B-jet & 0.39 \\
Lepton $p_{T}$ & 0.19 \\
Pileup & 0.95 \\
Calibration & 0.17 \\
\hline Total & 3.7 \\
\hline
\end{tabular}

Table 7.1: Total systematic uncertainties on $M_{t o p}$ for the $\tau+$ jets channel.

to account for changes in the event acceptance due to the rescaled jet energies. Recall that the lead background source (QCD multijets) is modeled using data (see Sec. 4.2). Therefore we do not shift the jet energies in the background samples. Although the acceptance differences cause the PE's to contain slightly different sets of events, the majority of events are the same across the shifted samples, so we treat the resulting measurements as highly correlated. For each jet energy correction, the systematic is taken to be half the difference between the up and down shifted samples. The measurement of $M_{t o p}$ with jets shifted by a $\pm 1 \sigma$ uncertainty along with the difference used to estimate the systematic and the systematic itself for each jet energy correction are listed in Tab. 7.2. We find the JES systematic uncertainty on $M_{t o p}$ to be $3.37 \mathrm{GeV}$ with the largest contribution coming from the absolute and out of cone corrections. This is expected since these two corrections have the largest fraction uncertainty per jet as is shown in Fig. 3.2 .

\subsection{Generator}

As detailed in Sec. 4.1, the signal $t \bar{t}$ MC used in this analysis is generated using PYTHIA. To evaluate the uncertainty due to the generator and the parton showering model used in PythiA, we compare the nominal result of the $M_{t o p}$ measurement with a result 


\begin{tabular}{|l||c|c|c|c|}
\hline \multicolumn{1}{|l||}{ Nominal $M_{\text {top }}$ value: } & \multicolumn{4}{c|}{$172.55 \mathrm{GeV}$} \\
\hline Correction & $+1 \sigma(\mathrm{GeV})$ & $-1 \sigma(\mathrm{GeV})$ & Diff $(\mathrm{GeV})$ & Syst $(\mathrm{GeV})$ \\
\hline Relative & 173.78 & 172.09 & 1.69 & 0.84 \\
Multiple Interaction & 172.79 & 172.57 & 0.24 & 0.12 \\
Absolute $(\eta)$ & 174.72 & 170.69 & 4.03 & 2.02 \\
Underling Event & 173.01 & 172.35 & 0.66 & 0.33 \\
Out of Cone & 175.58 & 170.59 & 4.97 & 2.49 \\
Splash Out & 173.38 & 172.30 & 1.08 & 0.54 \\
\hline Total & - & - & - & 3.37 \\
\hline
\end{tabular}

Table 7.2: Summary of the JES systematic uncertainty on the measurement of $M_{\text {top }}$ in the $\tau+$ jets decay channel. The $M_{\text {top }}$ measurement with a $\pm 1 \sigma$ is given for each jet correction. The systematic (Syst) uncertainty is taken to be half the total difference (Diff) between the shifted measurement (or the largest shift from the nominal measurement in the case of the multiple interaction correction). The final row shows the sum in quadrature of the uncertainty from each correction which is taken as the total JES systematic uncertainty on the measurement of $M_{t o p}$.

from a large sample of $t \bar{t}$ events generated with the HERwIG MC generator. Both the Pythia and Herwig MC samples are generated with a top quark mass of $172.5 \mathrm{GeV}$. We take the difference between the two measurements to be the systematic uncertainty from the MC generator model. However, since the two MC samples are made up of separate events, we consider possible statistical fluctuations on each $M_{\text {top }}$ measurement that could mask a larger systematic uncertainty if one or both measurements were to statistically fluctuate toward the nominal value.

As discussed in Sec. 6.6, because we use event resampling to generate PE's, the statistical uncertainty on the $M_{t o p}$ measurements will be artificially small. To properly measure the statistical uncertainty on the $M_{\text {top }}$ measurement we either use the bootstrap method described in App. F or build 1 large PE using all the signal events in the MC sample. We choose to use the 1 large PE method when evaluating systematic uncertainties since it returns roughly an equivalent answer as the bootstrap method but is much faster to run. However, since we do not have enough independent background events to properly build the single large PE with as many signal MC events as possible, we resample the background events in making the PE. For this reason, although we trust that the single large PE accurately estimates the uncertainty on the top quark 


\begin{tabular}{|l||c|c|c|}
\hline Source & PYTHIA $(\mathrm{GeV})$ & HeRWIG $(\mathrm{GeV})$ & Diff $(\mathrm{GeV})$ \\
\hline MC Generator & $172.30 \pm 0.34$ & $172.53 \pm 0.36$ & $0.23 \pm 0.50$ \\
\hline \hline Systematic $(\mathrm{GeV})$ & & & 0.50 \\
\hline
\end{tabular}

Table 7.3: Overview of the calculation of the MC Generator systematic uncertainties for the $M_{t o p}$ measurement in the $\tau+$ jets channel. The systematic uncertainty is taken to be the difference between the measurements made with PyTHIA and HeRWig MC samples (Diff). However, since the statistical uncertainty on the difference is larger than the actual difference between the measurements, we take the systematic uncertainty to be the statistical uncertainty on the difference between the $M_{t o p}$ measurements.

mass measurement, we take the central value of the systematic uncertainty on the $M_{\text {top }}$ measurement from the difference between the samples measured with an ensemble of PE's. We choose this because the central value from the 1 large PE measurement may be slightly biased due to the resampling of background events. The resulting $M_{t o p}$ measurements from both Pythia and Herwig MC generators can be seen in Tab. 7.3. Since the statistical uncertainty on the difference between the two measurements is greater than the difference itself, we take the systematic uncertainty from the MC generator model to be equal to the statistical uncertainty on the difference to ensure that we do not underestimate the systematic uncertainty because of statistical fluctuations on the $M_{t o p}$ measurements. We find the MC generator systematic uncertainty on $M_{t o p}$ is $0.50 \mathrm{GeV}$.

\subsection{Initial and Final State Radiation}

We consider an uncertainty on the $M_{t o p}$ measurement as a result of mismodeling the amount of ISR or FSR in the MC as described in Sec. 5.4.1. This radiation affects the $M_{t o p}$ measurement by either altering the jet multiplicity in the event (from extra radiated jets) or the jet energy spectrum (when the extra radiation falls within the jet cone of an existing $t \bar{t}$ daughter jet). Pyтнia $t \bar{t}$ MC samples are generated with the amount of ISR and FSR simultaneously scaled up or down by the $1 \sigma$ uncertainty as defined in Sec. 5.4.1. We then evaluate the ISR/FSR systematic uncertainty on the top quark mass by measuring $M_{t o p}$ with both MC samples. Just as in the MC generator 


\begin{tabular}{|l||c|c|c|}
\hline \multicolumn{1}{|c||}{ Nominal value of $M_{\text {top }}$} & \multicolumn{3}{c|}{$172.30 \pm 0.34 \mathrm{GeV}$} \\
\hline & More $(\mathrm{GeV})$ & Less $(\mathrm{GeV})$ & Diff $(\mathrm{GeV})$ \\
\hline IFSR & $172.69 \pm 0.58$ & $172.53 \pm 0.53$ & $0.39 \pm 0.67$ \\
\hline Systematic $(\mathrm{GeV})$ & - & - & 0.34 \\
\hline
\end{tabular}

Table 7.4: Details of the ISR/FSR systematic uncertainty on the measurement of $M_{\text {top }}$ in the $\tau+$ jets decay channel. The nominal $M_{\text {top }}$ measurement is given along with measurements made with $t \bar{t}$ MC samples with ISR and FSR turned simultaneously up (More) or down (Less). Since both ISR/FSR varied samples return measurements shifted in the same direction from the nominal value, we take the systematic uncertainty on the $M_{\text {top }}$ measurement to be the half the greatest difference from nominal (Diff). The largest difference from nominal is listed in the final column. As the statistical uncertainty on this difference is larger than the difference itself, the systematic uncertainty on the $M_{t o p}$ measurement given in the last row is taken to be half the statistical uncertainty on the difference rather than half the difference.

systematic in Sec. 7.4 these measurements are done with separate MC samples so we consider the statistical uncertainty of each measurement. The More and Less I/FSR samples are treated as $\pm 1 \sigma$ shifts on the effect from I/FSR mismodeling. The measured values of $M_{t o p}$ for varying ISR/FSR are listed in Tab. 7.4. We observe that the More and Less samples both measure a higher top quark mass than the nominal value of $172.30 \mathrm{GeV}$. As a result, we take the systematic to be half the greatest shift from the nominal value. Again, we find that the uncertainty on this difference is greater than the difference itself, so we take half the uncertainty rather than half the difference as the systematic which gives an uncertainty on $M_{\text {top }}$ of $0.34 \mathrm{GeV}$.

\subsection{Parton Distribution Functions}

The components of the PDF uncertainty are previously described in Sec. 5.4.1 with the main difference being that we separate the uncertainty due to the fraction of $g g$ fusion produced $t \bar{t}$ events into its own category to make the measurement easier to combine with other CDF analyses. We use the same reweighting schemes described in Sec. 5.4.1 to evaluate the uncertainty on the CTEQ PDF's and the uncertainty from different values of $\Lambda_{Q C D}$. We again find the central values of the $M_{t o p}$ measurement obtained with the CTEQ5L [85] and MRST72 [87] PDF's to be consistent, so the first component 
of the PDF uncertainty on $M_{t o p}$ is taken from the uncertainty measured over the 20 sets of CTEQ eigenvectors. The quadrature sum of this uncertainty with the difference in the $M_{t o p}$ measurements obtained using MRST72 and MRST75 which are derived with different values of $\operatorname{Lambda_{QCD}}$ is taken to be the total PDF uncertainty on $M_{t o p}$. We evaluate this uncertainty to be $0.12 \mathrm{GeV}$.

\subsection{Fraction of $g g \rightarrow t \bar{t}$ Produced Events}

As detailed in Sec. 1.3, $t \bar{t}$ pairs are produced either through $q \bar{q}$ annihilation or gluongluon $(g g)$ fusion. At the center-of-mass energy of the Tevatron, $q \bar{q}$ annihilation dominates the production of $t \bar{t}$ pairs. The $t \bar{t}$ Pythia MC we use produces $t \bar{t}$ pairs from gg fusion about $5 \%$ of the time which is the LO expectation. Additionally, the Mahlon Parke ME used in the mass measurement described in Sec. 6.3.1 assumes $q \bar{q}$ production of the $t \bar{t}$ pair. We consider a systematic uncertainty from NLO effects which predict as many as $20 \%$ of events produced from gg fusion [17]. To estimate this uncertainty, we reweight the events to bring the effective fraction of $t \bar{t}$ events from gg fusion production from $5 \%$ to $20 \%$. Table 7.7 shows the results of the gg fraction systematic uncertainty evaluation. We find the systematic uncertainty on the $M_{\text {top }}$ measurement as a result of fraction of $g g \rightarrow t \bar{t}$ production to be $0.17 \mathrm{GeV}$.

\subsection{Color Reconnection}

The MC we use in this analysis does not account for color charge interactions between the final state particles and the beam remnants defined as the partons in the initial interacting protons and anti-protons which are not involved in the interaction. Such effects are called "Color Reconnection" effects and are modeled in a new version of Pythia. This new Pythia model and the method used to evaluate the color reconnection systematic are described in Sec. 5.4.1. The systematic is taken as the difference in $M_{\text {top }}$ measurements with $\mathrm{MC}$ samples with and without color reconnection effects. As the samples are independently generated, the statistical uncertainty on the $M_{\text {top }}$ measurements is also considered. Tab. 7.8 gives the details of this systematic uncertainty 


\begin{tabular}{|l||c|c|c|c|}
\hline Eigenvector & Up $(\mathrm{GeV})$ & Down $(\mathrm{GeV})$ & Diff $(\mathrm{GeV})$ & Syst $(\mathrm{GeV})$ \\
\hline 1 & 174.40 & 174.42 & 0.02 & 0.010 \\
2 & 174.36 & 174.39 & 0.03 & 0.015 \\
3 & 174.41 & 174.38 & 0.03 & 0.015 \\
4 & 174.39 & 174.36 & 0.03 & 0.015 \\
5 & 174.43 & 174.41 & 0.02 & 0.010 \\
6 & 174.41 & 174.40 & 0.01 & 0.005 \\
7 & 174.39 & 174.42 & 0.03 & 0.015 \\
8 & 174.40 & 174.38 & 0.02 & 0.010 \\
9 & 174.41 & 174.38 & 0.03 & 0.015 \\
10 & 174.41 & 174.42 & 0.01 & 0.005 \\
11 & 174.42 & 174.41 & 0.01 & 0.005 \\
12 & 174.41 & 174.36 & 0.05 & 0.025 \\
13 & 174.37 & 174.38 & 0.01 & 0.005 \\
14 & 174.39 & 174.40 & 0.01 & 0.005 \\
15 & 174.40 & 174.37 & 0.03 & 0.015 \\
16 & 174.51 & 174.36 & 0.15 & 0.075 \\
17 & 174.36 & 174.39 & 0.03 & 0.015 \\
18 & 174.39 & 174.41 & 0.02 & 0.001 \\
19 & 174.40 & 174.39 & 0.01 & 0.005 \\
20 & 174.42 & 174.41 & 0.01 & 0.005 \\
\hline Total & - & - & - & 0.09 \\
\hline & CTEQ5L & MRST72 & Diff & Syst \\
\hline & 174.39 & 174.39 & 0.00 & 0.00 \\
\hline Systematic & - & - & - & 0.09 \\
\hline
\end{tabular}

Table 7.5: Breakdown of the first part of the PDF systematic uncertainty for $M_{t o p}$ in the $\tau+$ jets decay channel. First, the $M_{\text {top }}$ measurements are given for each set of CTEQ eigenvectors along with the difference between the measurements (Diff), and the resulting systematic (Syst) which is taken to be half the difference. These twenty systematic uncertainties are summed in quadrature to give a total systematic uncertainty of $0.09 \mathrm{GeV}$. Next, we check for differences between the CTEQ5L and MRST72 PDF models. We find that the two models give the same $M_{\text {top }}$ measurement. We take $0.09 \mathrm{GeV}$, the larger of these two effects, to be the first part of the PDF systematic uncertainty on $M_{t o p}$. 


\begin{tabular}{|l||c|c|c|}
\hline & - & - & Syst $(\mathrm{GeV})$ \\
\hline Part 1 & - & - & 0.09 \\
\hline & MRST72 $(\mathrm{GeV})$ & MRST75 $(\mathrm{GeV})$ & Syst $(\mathrm{GeV})$ \\
\hline Part 2 & 174.39 & 174.47 & 0.08 \\
\hline Total & - & - & 0.12 \\
\hline
\end{tabular}

Table 7.6: Details of the PDF systematic uncertainty for $M_{t o p}$ in the $\tau+$ jets decay channel. The first part (Part 1) is taken from Tab. 7.5. The second part is calculated as the difference in $M_{\text {top }}$ measurements using different values of $\Lambda_{Q C D}$ represented by the MRST72 and MRST75 PDF's. We take the second part of the systematic to be the difference in the result between the two measurements, giving a systematic of $0.08 \mathrm{GeV}$. Summing these two systematic in quadrature gives the total systematic uncertainty on $M_{\text {top }}$ due to PDF uncertainties of $0.12 \mathrm{GeV}$.

\begin{tabular}{|l||c|c|c|}
\hline & $5 \%$ gg $(\mathrm{GeV})$ & $20 \%$ gg $(\mathrm{GeV})$ & Diff $(\mathrm{GeV})$ \\
\hline Pythia $t \bar{t} \mathrm{MC}$ & 174.39 & 174.56 & 0.17 \\
\hline Systematic $(\mathrm{GeV})$ & - & - & 0.17 \\
\hline
\end{tabular}

Table 7.7: Details of the top quark mass measurement systematic uncertainty due to the fraction of $t \bar{t}$ pairs produced by gg fusion in the $\tau+$ jets decay channel. The $5 \%$ measurement is simply the nominal value measured with the standard PYTHIA MC sample with a top quark mass of $175.0 \mathrm{GeV}$. We then reweight the events in this sample so that the effective fraction of $t \bar{t}$ pairs produced by gg fusion is $20 \%$. The systematic uncertainty on the $M_{\text {top }}$ measurement is simply the difference between the two measured $M_{t o p}$ values. 


\begin{tabular}{|l||c|c|c|}
\hline Source & Apro $(\mathrm{GeV})$ & ACRpro $(\mathrm{GeV})$ & Diff $(\mathrm{GeV})$ \\
\hline Color Recon. & $172.17 \pm 0.36$ & $172.46 \pm 0.35$ & $0.29 \pm 0.50$ \\
\hline Total & - & - & 0.50 \\
\hline
\end{tabular}

Table 7.8: Details on the color reconnection systematic uncertainties on $M_{t o p}$ measurement in the $\tau+$ jets channel. The systematic is taken as the difference in $M_{\text {top }}$ measurements made with two different MC samples generated with (ACRpro) and without (Apro) color reconnection effects.

which is evaluated to be $0.50 \mathrm{GeV}$.

\subsection{Background Fraction}

We estimate the contribution of each background source in Sec. 4.3 . These contributions are handled in the mass measurement through the introduction of a background probability calculated for each event. To test and calibrate the method, we use ensembles of PE's with fully simulated backgrounds. The number of events from each background source is taken from the expectation described in Tab. 4.4. However, the effect of the uncertainty on the expectation for each background source is considered when measuring the systematic uncertainty on the top quark mass. To account for this uncertainty, we run PE's with the number of events from each background source fluctuated up and down by its $1 \sigma$ uncertainty. This is done for each of the main backgrounds: QCD multijets, $W+\mathrm{bb}, W+\mathrm{cc}, W+\mathrm{c}$, and $W+$ light flavor. The systematic uncertainty on $M_{t o p}$ is measured for each background source, and then the total uncertainty is taken from summing each contribution in quadrature giving a value of $0.47 \mathrm{GeV}$. For a breakdown of this uncertainty, see Tab. 7.9. We find that the largest contribution to this systematic uncertainty comes from the uncertainty on the estimated number of QCD multijet events. This is expected as the QCD multijet background is the by far the largest background source and has the most uncertainty on its estimated contribution. 


\begin{tabular}{|l||c|c|c|c|}
\hline \multicolumn{1}{|l||}{ Nominal } & \multicolumn{4}{c|}{$172.30 \mathrm{GeV}$} \\
\hline Source & $+1 \sigma$ & $-1 \sigma$ & Diff $(\mathrm{GeV})$ & Systematic $(\mathrm{GeV})$ \\
\hline Wbb & 172.20 & 172.25 & 0.10 & 0.05 \\
Wcc & 172.42 & 172.37 & 0.12 & 0.06 \\
Wc & 172.30 & 172.37 & 0.07 & 0.035 \\
Wlf & 172.16 & 172.38 & 0.22 & 0.11 \\
QCD & 171.76 & 172.65 & 0.89 & 0.445 \\
\hline Total & - & - & - & 0.47 \\
\hline
\end{tabular}

Table 7.9: Details for the calculation of the background systematic uncertainties on $M_{t o p}$ in the $\tau+$ jets channels. The top quark mass is measured with the contribution from each of the 5 major background sources fluctuated up and down by its $1 \sigma$ uncertainty. The systematic uncertainty is taken to be half the difference (Diff) between the up and down measurements or between the largest shift from nominal for each background source. In the final row, the total systematic uncertainty is given by taking the sum in quadrature of the uncertainty from each source.

\subsection{Statistics}

After applying all of the calibration functions, we fit the mass residual with a function which is constant versus $M_{\text {top }}$ and find it to be $-0.005 \pm 0.14 \mathrm{GeV}$ as can be seen in Fig. 6.27. The $0.14 \mathrm{GeV}$ uncertainty is due to the limited statistics of the MC samples, and we assign this uncertainty on the top quark mass measurement.

\subsection{B Jet Energy Scale}

We consider three sources due to the systematic uncertainty on the measurement of $b$ quark jets energies as described in [97]. Since all the measurements for the systematic uncertainties come from either reweighting events or shifting the energies of the $b$ quark jets in selected events, all $M_{\text {top }}$ measurements use the same events and any changes in the measured mass mean is attributed to the systematic uncertainty. As a result, we do not need to account for the statistical uncertainty of each top quark mass measurement used to estimate the systematic uncertainty.

First, we consider a systematic uncertainty to account for the Bowler parameters used in the PyThIA fragmentation model of the jets [98] 97. We reweight events using Bowler parameters derived from LEP and SLD data [99]. The measurements of $M_{\text {top }}$ 
are made with each set of reweighted events. The uncertainty is taken to be the largest difference between the measurements made with the LEP and SLD reweighting and the original measurement made with unweighted MC events. Next, we consider the shift caused by a $1 \sigma$ shift in the semi-leptonic branching ratios in the decay of heavy flavor partons formed by $b$ and $c$ quarks [97]. Again, we reweight events with these ratios shifted up and down by $1 \sigma$ and take the uncertainty to be half the total difference between the shifted measurements.

Finally, we consider a systematic uncertainty due to the $b$ jet energy. To measure this uncertainty, we shift the energies of all jets matched to $b$ quarks up and down by $1 \%$. Studies at CDF have shown that the b-tagging efficiency will not be largely affected by a $1 \%$ shift in the b-jet energy scale, so we do not consider changes in the event selection caused by such a shift. We then measure the systematic from a $1 \%$ shift in the $b$ jet energy scale to be half the difference between the two shifted $M_{t o p}$ measurements. Since a $1 \%$ shift in calorimeter response is estimated to provide a $0.2 \%$ shift on the $b$ jet energy scale [97, we take the final systematic to be 0.2 times the systematic measured for a $1 \%$ shift in the $b$ jet energy scale. For a breakdown of these three components and the resulting $b$ jet systematic uncertainty, see Tab. 7.10. Combining these three effects, we estimate the b-jet energy scale systematic on the top quark mass measurement to be $0.39 \mathrm{GeV}$.

\subsection{Lepton Energy Scale}

Just as shifts in the jet energies can cause the reconstructed top quark mass to shift, a shift in the lepton energy scale can also cause changes in the measured top quark mass. For $\tau+$ jets events, we first determine the uncertainty on the $\tau$ energy. From a previous study performed in [100], $W$ 's decaying to a $\tau$ and $\nu$ are selected using a very similar set of $\tau$ requirements to those given in Tab. 3.1. By requiring high $E_{\mathrm{T}}$, a very clean sample of $W \rightarrow \tau \nu$ events with little QCD multijet contribution is used to obtain the $\tau P_{T}$ distribution in the data and from expected MC. The $\tau$ energy is shifted, and Kolmogorov-Smirnov (KS) tests [101] are performed to check for agreement between the data and MC. This study shows that a 1\% energy shift correctly estimated the $\tau$ 


\begin{tabular}{|c|c|c|c|c|}
\hline Nominal & \multicolumn{4}{|c|}{$174.39 \mathrm{GeV}$} \\
\hline & B Frag SLD & B Frag LEP & Diff $(\mathrm{GeV})$ & Syst (GeV) \\
\hline$t \bar{t} \mathrm{MC}$ & 174.67 & 174.60 & 0.28 & 0.28 \\
\hline & SemiLep BR up & SemiLep BR down & Diff $(\mathrm{GeV})$ & Syst $(\mathrm{GeV})$ \\
\hline$t \bar{t} \mathrm{MC}$ & 174.13 & 174.56 & 0.43 & 0.22 \\
\hline & $b$ jet $\operatorname{En}+1 \%$ & $b$ jet En $-1 \%$ & Diff $(\mathrm{GeV})$ & Syst (GeV) \\
\hline Nominal & \multicolumn{4}{|c|}{$172.55 \mathrm{GeV}$} \\
\hline$t \bar{t} \mathrm{MC}$ & 173.66 & 172.08 & 1.58 & 0.16 \\
\hline Total & - & - & - & 0.39 \\
\hline
\end{tabular}

Table 7.10: Measurements of $M_{t o p}$ used to evaluate the three components of the total systematic uncertainty are shown. First, the uncertainty (Syst) due to the Bowler parameters is taken as the largest difference (Diff) between the nominal and the two reweighted sets of events. Next, the systematic is shown for a $1 \sigma$ uncertainty in the semileptonic branching ratio (SemiLep BR). Both of these are evaluated using $t \bar{t}$ MC with $M_{t o p}=175.0 \mathrm{GeV}$. Finally, the systematic uncertainty due to the calorimeter energy scale is measured to be $0.2 \%$ of the difference observed for a $1 \%$ shift in the calorimeter energy scale. These measurements are made with $t \bar{t} \mathrm{MC}$ with $M_{\text {top }}=172.5$ $\mathrm{GeV}$. The three uncertainties are summed in quadrature to evaluate the total b-jet energy scale systematic on $M_{t o p}$.

energy uncertainty [100].

We rerun the event selection with the energy of all identified $\tau$ 's shifted up and down by $1 \%$ to account for both changes from the energy scale and event acceptance. Although the event selection changes slightly with this shift, the majority of events in the shifted samples are still the same, so changes in $M_{\text {top }}$ measurements are due to the change in lepton energy, and we ignore the statistical uncertainty on these measurements. Also, it is worth noting that the detector measures the $\tau$ energy from the hadronic decay components of the $\tau$ lepton. Since, we are measuring the systematic uncertainty due to the $\tau$ energy scale in the CDF detector, we shift the energy of the $\tau$ "jet" before applying the neutrino recovery method described in Sec. 6.1. We find both shifted results to be greater than the nominal value, as seen in Tab. 7.11. We take the systematic uncertainty on the lepton energy scale to be half the largest shift from the nominal value which gives a $\tau$ energy scale systematic uncertainty on $M_{\text {top }}$ of $0.19 \mathrm{GeV}$. 

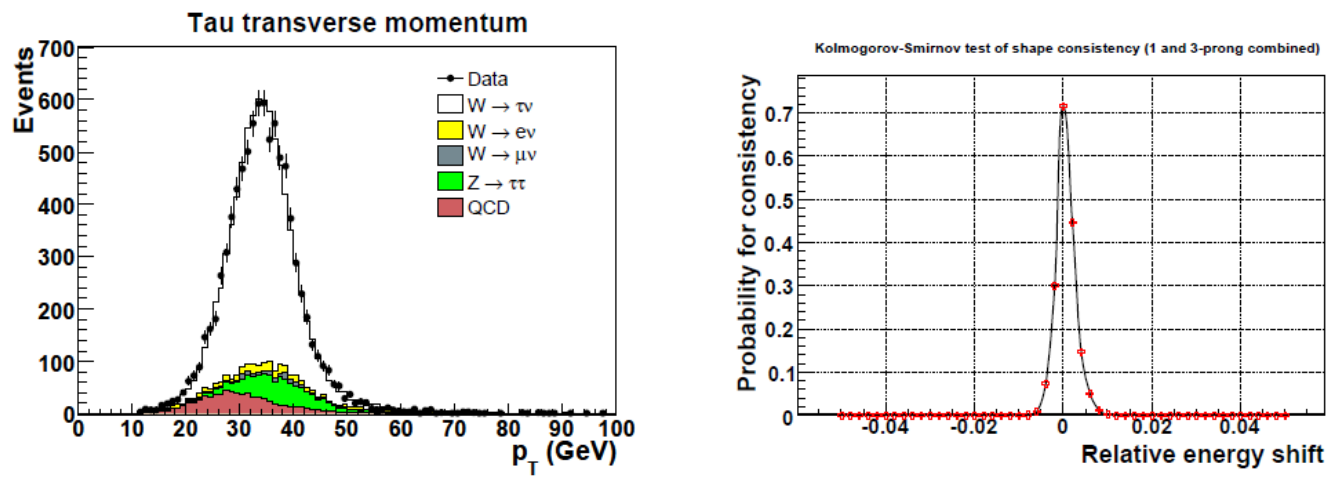

Figure 7.1: On the left is shown the distribution of $\tau P_{T}$ in data and MC events selected with $E_{\mathrm{T}}>30 \mathrm{GeV}$ to reduce the QCD multijet contribution taken from [100]. The $\tau$ energy spectrum is then shifted, and a KS test is performed to measure the agreement in the data and $\mathrm{MC}$. The results of the $\mathrm{KS}$ test for various energy scale shifts can be seen on the right again taken from [100]. We conclude from this test that a $1 \%$ shift on the $\tau$ energy corresponds to a $1 \sigma$ shift in the $\tau$ energy scale. [100].

\begin{tabular}{|l||c|c|c|c|}
\hline \multicolumn{1}{|l||}{ nominal } & \multicolumn{4}{|c|}{$172.55 \mathrm{GeV}$} \\
\hline Lepton & Energy $+1 \%(\mathrm{GeV})$ & Energy $-1 \%(\mathrm{GeV})$ & Diff $(\mathrm{GeV})$ & Syst $(\mathrm{GeV})$ \\
\hline Tau & 172.79 & 172.93 & 0.38 & 0.19 \\
\hline
\end{tabular}

Table 7.11: Details for the lepton energy scale systematic uncertainty for the $M_{t o p}$ measurement in the $\tau+$ jets decay channel. We shift the $\tau$ energies in $t \bar{t} \mathrm{MC}$ up and down by $1 \%$ and reselect events and measure $M_{t o p}$. As both new measurements differ in the same direction from the nominal measurement, we take half the greatest difference (Diff) from the nominal value as the systematic uncertainty (Syst) on $M_{t o p}$. 


\subsection{Pile-up}

We consider as a source of uncertainty the possibility of identifying extra energy in $t \bar{t}$ event from interactions between partons in the colliding $p$ and $\bar{p}$ other than the two involved in the hard collision which produces the $t \bar{t}$ pair. We refer to this effect as pile-up. We measure the uncertainty due to pile-up effects in two different ways and, to be conservative, chose to use the larger of the two measurements as the systematic uncertainty on $M_{t o p}$. The first method we will call known "mismodeling" as it describes the uncertainty due to the difference in the luminosity profile between the data and the $\mathrm{MC}$ events. The second we will call "unknown mismodeling" as it describes a possible mismodeling in the minimum bias events which we use to model additional parton interactions within an event.

The luminosity profile is the data is known to not match that in the $t \bar{t} \mathrm{MC}$. This effect can be observed by checking the number of vertices observed in each event $\left(N_{v t x}\right)$, as shown in Fig. 7.2, since the luminosity profile directly affects this distribution. As the instantaneous luminosity increases, the number of particles at the collision point increase. This leads to more interactions, and thus more event vertices. To estimate this uncertainty, we reweight events in the $t \bar{t} \mathrm{MC}$ sample so that the $N_{v t x}$ distribution matches that in the data. The uncertainty is the difference between the results with and without these weights.

"Unknown mismodeling" refers to a possible mismodeling of the events we use to model soft parton interactions. The term soft interaction is used to describe any interaction between partons in the colliding $p$ and $\bar{p}$ which do not lead to $2 \rightarrow 2$ hard scattering events. These interactions are a constant background in the collisions we are interested in, but unfortunately, they are governed by non-perturbative QCD which is poorly modeled with MC. To estimate the contribution from these events we select events which have a minimum bias on the selection sample. Generally, we impose selection requirements such as a minimum jet $E_{T}$ which bias the selection toward $2 \rightarrow 2$ hard scattering interactions. By relaxing these requirements, we allow more soft parton interaction events the sample. In practice, we use a trigger which selects events from 


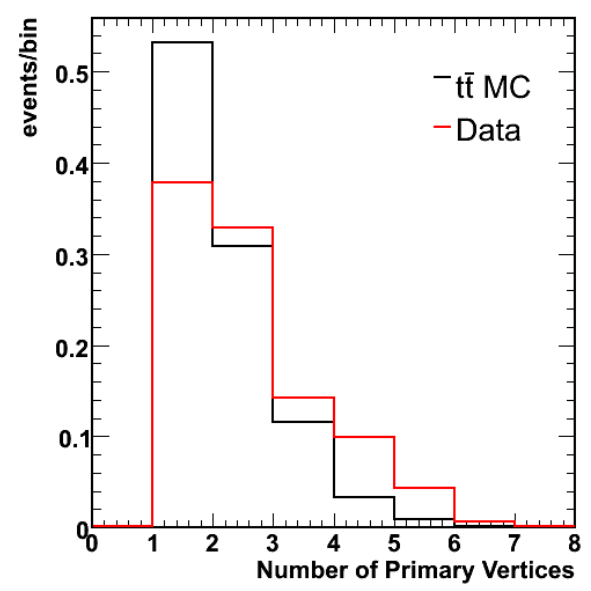

Figure 7.2: Number of primary vertices in data (red) and $t \bar{t} \mathrm{MC}$ (black). Data events have an average of 2.24 vertices, and $t \bar{t} \mathrm{MC}$ events have an average of 1.68 primary vertices.

all possible types of interactions at a rate which is proportional to their production. This gives data events which have minimum bias towards hard scattering interactions. We then use this sample to model the background of soft parton interactions. The "unknown mismodeling" uncertainty refers to any uncertainty from mismodeling of these soft interactions.

It has been found that the jet energy response versus the number of interactions in an event in $t \bar{t} \mathrm{MC}$ events does not match the observed effect in minimum bias events. Unfortunately, there is not enough statistics in $t \bar{t}$ data events to check if this effect is seen there as well. Instead, we use the discrepancy observed in the MC to estimate the the size of the possible effect of mismodeling of the minimum bias events. From MC studies, the observed slope of the jet energy response is approximately $250 \mathrm{MeV}$ per jet per vertex. We know that the response of the multiple interaction jet energy scale correction is $107 \mathrm{MeV}$ per jet per vertex. As a result, we model the uncertainty by scaling the multiple interaction jet correction systematic uncertainty up by a factor of $\frac{250}{107}=2.3$. However, this measurement is complicated by the fact that the multiple interaction correction depends on $N_{v t x}-1$ and is evaluated with $\mathrm{MC}$ which has the wrong $N_{v t x}$ distribution. To fix this, we reweight the uncertainty by the average number 


\begin{tabular}{|c|c|c|c|}
\hline \multicolumn{4}{|c|}{ Known Mismodeling } \\
\hline & Nominal $(\mathrm{GeV})$ & $N_{\text {vrtx }}$ Weighted $(\mathrm{GeV})$ & Diff $(\mathrm{GeV})$ \\
\hline & 172.30 & 171.35 & 0.95 \\
\hline \multicolumn{4}{|c|}{ Unknown Mismodeling } \\
\hline$\left\langle N_{\text {vrtx }}^{\text {data }}\right\rangle$ & $\left.<N_{\text {vrtx }}^{\text {MC }}\right\rangle$ & SF & Systematic \\
\hline 2.244 & 1.680 & 0.267 & 0.50 \\
\hline Systematic & \multicolumn{3}{c}{0.95} \\
\hline
\end{tabular}

Table 7.12: Details for the calculation of the pile-up systematic uncertainty for $M_{t o p}$ measured in the $\tau+$ jets decay channel. The "known mismodeling" is taken as the difference (Diff) between the $M_{t o p}$ measurements made with $t \bar{t} \mathrm{MC}$ and the same $t \bar{t}$ MC reweighted to match the luminosity profile observed in the data. The "unknown mismodeling" is derived from the average number of vertices observed in the events $\left(<N_{v t x}>\right)$ and the multiple interaction JES systematic uncertainty on the top quark mass which was measured to be 0.12 in Sec. 7.3 . The systematic is evaluated as $\frac{\left\langle N_{v t x}^{d a t a}\right\rangle-1}{\left\langle N_{v t x}^{M L C}\right\rangle-1}$ times the SF of 0.267 . We take the larger of the two as the pileup systematic uncertainty on $M_{t o p}$.

of vertices per data event minus 1 divided by the average number of vertices in the MC event minus 1. Altogether, the unknown mismodeling pileup systematic is estimated as $\left(2.3 \cdot \frac{N_{v r t x}^{\text {data }}-1}{N_{v r t x}^{M C}-1}\right)$ times the multiple interaction systematic measured in Sec. 7.3

As can be seen in Tab. 7.12, the "known mismodeling" piece of the pile-up systematic uncertainty is larger than the "unknown mismodeling" piece, so we take the known mismodeling uncertainty of $0.95 \mathrm{GeV}$ as the total pile-up systematic uncertainty on the top quark mass measurement.

\subsection{4 $M_{\text {top }}$ Measurement Calibration}

The final systematic uncertainty we consider comes from the calibration functions for the top quark mass measurement we derived in Sec. 6.6. To calibrate the mass measurement, we fit a 1st order polynomial to the distribution of the output measured mass versus the input mass in Fig. 6.24. We estimate the calibration uncertainty by shifting the derived correction function within the $1 \sigma$ uncertainties of the resulting fit. We then reperform the top quark mass measurement on $t \bar{t} \mathrm{MC}$ with $M_{t o p}$ of $172.5 \mathrm{GeV}$ using these two different calibration functions, and we take half the total shift as the uncertainty. Table 7.13 lists results from each calibration function. We find the systematic 


\begin{tabular}{|l|c|c|c|}
\hline Nominal & \multicolumn{3}{|c|}{$172.30 \mathrm{GeV}$} \\
\hline$+1 \sigma$ & $-1 \sigma$ & Diff $(\mathrm{GeV})$ & Systematic $(\mathrm{GeV})$ \\
\hline 172.36 & 172.02 & 0.34 & 0.17 \\
\hline
\end{tabular}

Table 7.13: Details for the calculation of the calibration systematic uncertainty for the $M_{\text {top }}$ measurement in the $\tau+$ jets decay channel. The calibration function is shifted up and down by its $1 \sigma$ uncertainty and the $M_{t o p}$ measurement is reperformed on $t \bar{t} \mathrm{MC}$ with $M_{\text {top }}$ equal to $172.5 \mathrm{GeV}$. The first two columns of the table give these measurements. We take half the total difference between these two measurements (Diff) to be the systematic uncertainty on the $M_{t o p}$ measurement (Syst).

uncertainty on the top quark mass measurement due to the measurement calibration to be $0.17 \mathrm{GeV}$. 


\section{Chapter 8}

\section{Conclusion}

We have successfully measured the $t \bar{t}$ pair production cross section and the top quark mass in the $\tau+$ jets decay channel at the CDF detector at the Tevatron. The cross section measurement is the first in this decay channel at CDF, and the top mass measurement is the first ever such measurement in $\tau+$ jets $t \bar{t}$ decays in the world. Both measurements are made with $2.2 \mathrm{fb}^{-1}$ of data. We find 41 candidate $\tau+$ jets events in this data sample of which we expect approximately half to be $t \bar{t}$ decays and the other half to be QCD multijet production. We find the cross section to be $8.8 \pm 3.3($ stat.) \pm 2.2 (syst.) pb assuming an $M_{\text {top }}$ of $172.5 \mathrm{GeV}$. This is in good agreement with the CDF average with $4.6 \mathrm{fb}^{-1}$ of $7.5 \pm 0.5 \mathrm{pb}$ assuming an $M_{\text {top }}$ of $172.5 \mathrm{GeV}$ [18] as well as the DØ measurement in the $\tau+$ jets decay channel with $1 \mathrm{fb}^{-1}$ of data of $6.9 \pm 2.2 \mathrm{pb}$ assuming a top quark mass of $170.0 \mathrm{GeV}$ [1]. The top quark mass measurement is made using a matrix element method. Analyses using this method use the maximum amount of information available in the event to measure the top quark mass and, as a result, are generally the most precise mass measurements. We find the top quark mass to be $172.7 \pm 9.3$ (stat.) \pm 3.7 (syst.) $\mathrm{GeV}$. This is also in good agreement with the Tevatron mass combination with $5.8 \mathrm{fb}^{-1}$ of $173.2 \pm 0.9 \mathrm{GeV}$ [16].

Both measurements show promise for significant reduction in their uncertainties if the full CDF dataset of $\sim 10 \mathrm{fb}^{-1}$ were to be used. The statistical uncertainty reduces as a factor of $\frac{1}{\sqrt{N}}$, where $\mathrm{N}$ is the number of events, and an increase in the amount of data by 4 or 5 times should reduce the statistical uncertainty on each measurement by roughly a factor of 2 . There is also reason to be optimistic regarding the systematic uncertainties for both measurement. The systematic uncertainty on the cross section measurement is dominated by the uncertainty on the QCD multijet fraction as shown 
in Tab. 5.2. Since this uncertainty is determined by comparing the data-driven QCD multijet model to QCD multijet dominated data, the uncertainty is limited by the statistics of the data-driven model. Therefore, with more data, this uncertainty is likely to reduce approximately as a statistical uncertainty would. As for the $M_{t o p}$ measurement, as seen in Tab. 7.1 the total systematic uncertainty is dominated by far by the JES uncertainty. Several $M_{t o p}$ measurements simultaneously measure $M_{t o p}$ and JES by taking advantage of the dijet mass from the hadronic side $W$ decay (see [53]). With only 41 data events, we found that this analysis was not sensitive to the JES through the $W$ mass, however, we expect the measurement to be sensitive once the dataset grows by about a factor of 4 . As a result, it is possible that with the full CDF dataset, this analysis would have the sensitivity to perform an in-situ jet energy scale calibration which would reduce the JES systematic uncertainty.

Neither measurement shows any evidence against the premise of lepton universality. Meanwhile, the success of these measurements show that we can use $\tau$ leptons for complicated analyses at hadron colliders. This is particularly noteworthy as, due to its higher mass, the $\tau$ may be a preferred decay channel for various possible new physics signals (such as a charged Higgs boson as described in [23]). Additionally, the use of the NN to reduce the QCD multijet background may be of interest for analyses at the LHC as the QCD multijet background is several orders of magnitude larger there than at the Tevatron. 


\section{Appendix A}

\section{Measurement from a Negative Log Likelihood Function}

In this analysis, we chose to work with the negative log of the calculated likelihood function. For a likelihood function based on Gaussian probabilities, the resulting negative log likelihood takes the form of a $2^{\text {nd }}$ order polynomial. This can be simply shown:

$$
\begin{aligned}
-\log \mathcal{L} & =-\log \left(A \exp \left(\frac{-[x-\mu]^{2}}{2 \sigma^{2}}\right)\right), \\
-\log \mathcal{L} & =\frac{[x-\mu]^{2}}{2 \sigma^{2}}+\log A
\end{aligned}
$$

where $\mathrm{A}$ is some constant factor, $\mu$ is the mean value of the parameter being measured, and $\sigma$ is the standard deviation of that parameter. As a result, we fit the negative log likelihood function with a $2^{\text {nd }}$ order polynomial of the form $A x^{2}+B x+C=0$. To find the central value of the parameter being measured, $\mu$, we simply minimize the negative $\log$ likelihood function by setting the first derivative of the polynomial to 0 and solving for $\mathrm{x}$. For a $2^{\text {nd }}$ order polynomial, this is:

$$
\begin{array}{r}
\frac{d}{d x}\left(A x^{2}+B x+C\right)=0, \\
2 A x+B=0, \\
x=-\frac{B}{2 A}=\mu .
\end{array}
$$

To calculate the statistical uncertainty on $\mu, \sigma$, we consider the form of the negative $\log$ likelihood function in Eqn. A.2. For simplicity sake, we can arbitrarily rescale the likelihood function so that its value is 0 at an $x=\mu$. Then the value of $\mathcal{L}$ at a point $\mathrm{N}$ 
standard deviations from the minimizing value of $\mathrm{x}, x=\mu+N \cdot \sigma$, is found to be:

$$
\begin{aligned}
-\log L_{\mu+N \sigma} & =\frac{[\mu+N \cdot \sigma-\mu]^{2}}{2 \sigma^{2}} \\
-\log L_{\mu+N \sigma} & =\frac{N^{2} \cdot \sigma^{2}}{2 \sigma^{2}} \\
-\log L_{\mu+N \sigma} & =\frac{N^{2}}{2}
\end{aligned}
$$

As a result, a $1 \sigma$ uncertainty on $\mu$ will be at the point that the likelihood function rises in value by $1 / 2$ unit above its minimum value, while a $2 \sigma$ uncertainty will be at the point that the likelihood function rises in value by $4 / 2$ units above its minimum, etc.

Assuming the likelihood minimizes at 0 , we can thus simply calculate the value of $\sigma$ by calculating $x_{\sigma}-\mu$, where $x_{\sigma}=\mu+\sigma$ and $\mu=-\frac{B}{2 A}$ :

$$
\begin{array}{r}
L_{1 \sigma}=L_{0}+\frac{1}{2} \\
A x_{\sigma}^{2}+B x_{\sigma}+C=A \mu^{2}+B \mu+C+\frac{1}{2} \\
A x_{\sigma}^{2}+B x_{\sigma}=A \cdot\left(\frac{B^{2}}{4 A^{2}}\right)+B \cdot \frac{-B}{2 A}+\frac{1}{2} \\
A x_{\sigma}^{2}+B x_{\sigma}=\frac{B^{2}}{4 A}-\frac{B^{2}}{2 A}+\frac{1}{2} \\
A x_{\sigma}^{2}+B x_{\sigma}+\frac{B^{2}-2 A}{4 A}=0 \\
x_{\sigma}=\frac{-B \pm \sqrt{B^{2}-\left(B^{2}-2 A\right)}}{2 A} \\
x_{\sigma}=-\frac{B}{2 A} \pm \frac{\sqrt{2 A}}{2 A} \\
x_{\sigma}=\mu \pm \frac{1}{\sqrt{2 A}} .
\end{array}
$$

So the standard deviation is found to be $\frac{1}{\sqrt{2 A}}$. 


\section{Appendix B Smeared Electron Test}

While the $\nu$ scan method described in Sec. 6.1 improves the energy resolution of the $\tau$, the reconstructed $\tau$ 's energy resolution still does not approximate a delta function like the energy resolution of an electron or a $\mu$. To estimate the effect on the top quark mass measurement from the spread of the energy resolution, we devise a test based on a previous version of this analysis which is designed to measure $M_{t o p}$ from $e$ and $\mu+$ jets events [53]. We start by fitting a Gaussian function to the energy resolution of $\tau$ 's selected from $t \bar{t} \rightarrow \tau+$ jets MC events. We then select a sample of $t \bar{t} \rightarrow$ electron + jets $\mathrm{MC}$ events. We make a second selection of these same electron + jets events with the electron energy resolution smeared by the Gaussian function from the $\tau$ sample. This process is demonstrated in Fig. B.1.

Next, we throw ensembles of PE's with the matrix element method to measure $M_{t o p}$ in the smeared and unsmeared electron + jets samples. The is done with three different top quark mass points $(170 \mathrm{GeV}, 175 \mathrm{GeV}$, and $180 \mathrm{GeV})$, and the smeared and unsmeared measurements are compared at each point. The results are shown in Tab. B.1. First, we do this with the background probability "turned off" in the likelihood function $\left(A_{b k g d}\right.$ is set to 0$)$. In this case, we find that the smearing of the lepton energy resolution in this model presents a relatively constant $2 \mathrm{GeV}$ shift on the central value. Next, we repeat the measurement with the background probability "turned on". Even though the events being considered are all $t \bar{t}$ signal events, the inclusion of the background probability helps smooth out fluctuations in the likelihood function from signal events which do not appear match the signal hypothesis (usually events in which the 4 jets do not match to the 4 partons). In this case, we find the smeared electrons give a constant $0.5 \mathrm{GeV}$ bias. In both the cases, the smearing of the lepton energy 
resolution has little to no effect on the statistical uncertainty of the measurement. As a result, any bias in the $\tau+$ jets $M_{t o p}$ measurement from the $\tau$ energy resolution can be removed during the method calibration (described in Sec. 6.6).
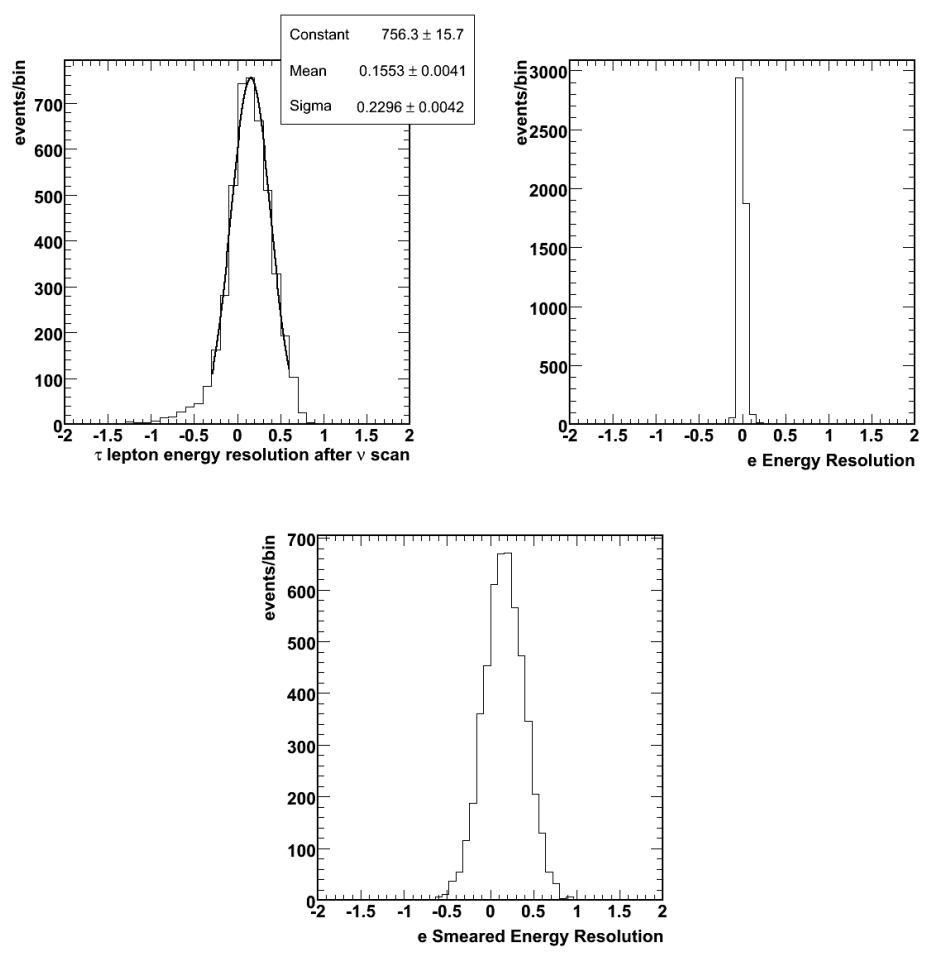

Figure B.1: Corrected $\tau$ energy resolution (upper left), electron energy resolution (upper right), and electron energy resolution after being smeared to match $\tau$ energy resolution (bottom). 


\begin{tabular}{|l|c|c|}
\hline Input Mass & Unsmeared Output Mass & Smeared Output Mass \\
\hline Signal Probability & & \\
\hline $170 \mathrm{GeV}$ & $170.5 \pm 0.5$ & $172.7 \pm 0.5$ \\
$175 \mathrm{GeV}$ & $174.7 \pm 0.3$ & $176.6 \pm 0.3$ \\
$180 \mathrm{GeV}$ & $179.4 \pm 0.5$ & $182.0 \pm 0.5$ \\
\hline Signal and Bkgd & & \\
\hline $170 \mathrm{GeV}$ & $169.9 \pm 0.5$ & $170.4 \pm 0.5$ \\
$175 \mathrm{GeV}$ & $173.8 \pm 0.3$ & $174.3 \pm 0.3$ \\
$180 \mathrm{GeV}$ & $179.4 \pm 0.5$ & $180.1 \pm 0.6$ \\
\hline
\end{tabular}

Table B.1: Results of smeared electron test. The top half of the table details the $M_{\text {top }}$ measurements using just the signal probability $\left(A_{b k g d}\right.$ set to 0$)$. The bottom half details the measurement with the same events, but the likelihood function includes the background probability. Despite these being signal events, the inclusion of the background probability smooths out the contribution from signal events which do not fit the signal assumption. 


\section{Appendix C}

\section{Kinematic Event Solution}

We present here the kinematic solution of a $t \bar{t} \rightarrow \tau+$ jet event based on measured detector quantities and the integrated variables. First we discuss the solution for all the particles from the hadronically decaying top. Then, we do the same for the leptonically decaying top. For the solution, we assume the top and anti-top quark masses are the same.

\section{C.1 Hadronic Side}

On the hadronic side of the equation, we have three jets. One of the light quark jet energies is covered by the integration, and we use it to solve for the other two. We demonstrate in Sec. 6.4 .2 that there are biases in the angles between the two light quark jets from the $\mathrm{W}$ decay and between the $W$ and the $b$ quark. To accont for this, we integrate over the variables $\cos \alpha_{12}$ and $\cos \alpha_{W b}$.

- Assumptions: $m_{j_{1}}=m_{j_{2}}=0, m_{b_{h a d}}=4.8 \mathrm{GeV}$

- Integrated variables: $m_{t o p_{h a d}}^{2}, m_{W_{h a d}}^{2}, p_{j_{1}}, \cos \alpha_{12}, \cos \alpha_{W b}$

- To Be Determined: $p_{j_{2}}, p_{b_{h a d}}$

Knowing the $W$ mass and the momentum of jet 1 allows us to easily calculate the second jet momentum:

$$
\begin{aligned}
m_{W_{h a d}}^{2} & =E_{W_{h a d}}^{2}-\vec{p}_{W_{h a d}}^{2} \\
m_{W_{h a d}}^{2} & =\left(E_{j_{1}}+E_{j_{2}}\right)^{2}-\left(\vec{p}_{j_{1}}+\vec{p}_{j_{2}}\right)^{2} \\
m_{W_{h a d}}^{2} & =m_{j_{1}}^{2}+m_{j_{2}}^{2}+2 p_{j_{1}} p_{j_{2}}\left(1-\cos \alpha_{12}\right) \\
p_{j_{2}} & =\frac{m_{W_{h a d}}^{2}}{2 p_{j_{1}}\left(1-\cos \alpha_{12}\right)} .
\end{aligned}
$$


From Eqn. C.4. we can now calculate $p_{j_{2}}$ from the integrated variables $m_{W_{h a d}}^{2}, p_{j_{1}}$, and $\cos \alpha_{12}$.

Now that we have the light quark momenta, we are prepared to calculate the momentum of the hadronic side $b$ quark.

$$
\begin{aligned}
& m_{t_{h a d}}^{2}=E_{t_{h a d}}^{2}-\vec{p}_{t_{h a d}}^{2} \\
& m_{t_{h a d}}^{2}=m_{W_{h a d}}^{2}+m_{b_{h a d}}^{2}+2\left(E_{W_{h a d}} E_{b_{h a d}}-\vec{p}_{W_{h a d}} \cdot \vec{p}_{b_{h a d}}\right) .
\end{aligned}
$$

To simplify things, we substitute $\mathrm{M}$ for the known quantity:

$$
M=\frac{\left(m_{t_{h a d}}^{2}-m_{W_{h a d}}^{2}-m_{b_{h a d}}^{2}\right)}{2} .
$$

We now have:

$$
\begin{aligned}
M & =E_{W_{h a d}} E_{b_{h a d}}-\vec{p}_{W_{h a d}} \cdot \vec{p}_{b_{h a d}} \\
M & =\left(p_{j_{1}}+p_{j_{2}}\right) E_{b_{h a d}}-\left(p_{j_{1}}+p_{j_{2}}\right) p_{b_{h a d}} \cos \alpha_{W b} \\
M & =P E_{b_{h a d}}-D p_{b_{h a d}}
\end{aligned}
$$

where:

$$
\begin{gathered}
P=p_{j_{1}}+p_{j_{2}}, \\
D=P \cos \alpha_{W b} .
\end{gathered}
$$

We now solve for $p_{b_{h a d}}$ :

$$
\begin{aligned}
M & +D p_{b_{h a d}}=P \sqrt{\left(m_{b_{h a d}}^{2}+p_{b_{h a d}}^{2}\right)}, \\
M^{2} & +2 M D p_{b_{h a d}}+D^{2} p_{b_{h a d}}^{2}=P^{2} m_{b_{h a d}}^{2}+P^{2} p_{b_{h a d}}^{2}, \\
0 & =\left(D^{2}-P^{2}\right) p_{b_{h a d}}^{2}+2 M D p_{b_{h a d}}+M^{2}-P^{2} m_{b_{h a d}}^{2}, \\
p_{b_{h a d}} & =\frac{-M D \pm P \sqrt{M^{2}+m_{b_{h a d}}^{2}\left(D^{2}-P^{2}\right)}}{\left(D^{2}-P^{2}\right)} .
\end{aligned}
$$

Before we move on to the leptonic side, we first check that the momentum of the hadronic side $b$ quark is positive. We then calculate $E_{b_{h a d}}=\sqrt{p_{b_{h a d}}^{2}+m_{b_{h a d}}^{2}}$. 


\section{C.2 Leptonic Side}

With the decay kinematics of the hadronically decaying top quark fully solved, we move onto the leptonically decaying top quark. We make a few assumptions here to simplify the equations and the integration due to time constraint. First, we assume that the $\tau$ lepton is massless. Since the $W$ mass is $80.4 \mathrm{GeV}$ and the $\tau$ mass is $1.8 \mathrm{GeV}, m_{W}^{2} \gg m_{\tau}^{2}$, and we find this assumption is valid. Second, we assume that the $t \bar{t}$ system has no initial transverse momentum.

- Known variables: $p_{\tau}, \theta_{\tau}, \phi_{\tau}, \theta_{b_{l e p}}, \phi_{b_{l e p}}$

- Assumptions: $m_{b_{l e p}}=4.8 \mathrm{GeV}, m_{\tau}=0, p_{t \bar{t}}^{x}=p_{t \bar{t}}^{y}=0$

- Integrated variables: $m_{W_{l e p}}^{2}$

- To Be Determined: $p_{b_{l e p}}, p_{\nu}, \theta_{\nu}, \phi_{\nu}$

First, we want to define a few variables for simplicity sake. Although we know the $\theta$ and $\phi$ angles for the lepton and jets, we prefer to work with the $\mathrm{x}, \mathrm{y}$, and z components of the momentum. To simplify the notation, we define the terms scn, ssn, and cn, where $\mathrm{n}$ can be $\mathrm{t}$ for the $\tau$ lepton, $\mathrm{bl}$ for the leptonic $b$ jet, or nu for the neutrino:

$$
\begin{gathered}
p_{n}^{x}=p_{n} \sin \phi_{n} \cos \theta_{n}=p_{n}(s c n) \\
p_{n}^{y}=p_{n} \sin \phi_{n} \sin \theta_{n}=p_{n}(s s n) \\
p_{n}^{z} \quad=p_{n} \cos \phi_{n}=p_{n}(c n) .
\end{gathered}
$$

Next, we want to define the variables $S_{x}\left(\operatorname{Sum} P_{x}\right)$ and $S_{y}$ from known quantities to account for the unknown $\mathrm{x}$ and $\mathrm{y}$ momentum of the leptonic side $b$ quark and the neutrino.

$$
S_{x}=p_{j_{1}}^{x}+p_{j_{2}}^{x}+p_{b_{h a d}}^{x}+p_{\tau}^{x}
$$

which gives:

$$
\begin{aligned}
p_{t \bar{t}}^{x} & =S_{x}+p_{b_{l e p}}^{x}+p_{\nu}^{x}=0 \\
S_{x} & =-p_{b_{l e p}}^{x}-p_{\nu}^{x}
\end{aligned}
$$


We similarly define $S_{y}$ :

$$
S_{y}=-p_{b_{l e p}}^{y}-p_{\nu}^{y}
$$

We can use these equations to define the neutrino momentum in terms of the $\nu$ angles and known quantities.

$$
\begin{aligned}
p_{b_{l e p}}^{x} & =-S_{x}-p_{\nu}^{x}, \\
p_{b_{l e p}}(s c b l) & =-S_{x}-p_{\nu}(s c n u) . \\
p_{b_{l e p}}^{y} & =-S_{y}-p_{\nu}^{y}, \\
p_{b_{l e p}}(s s b l) & =-S_{y}-p_{\nu}(\text { ssnu }) .
\end{aligned}
$$

Combining Eqns.C.25 and (C.27) and eliminating $p_{b_{l e p}}$ gives:

$$
p_{\nu}((\operatorname{scnu})(s s b l)-(\operatorname{ssnu})(s c b l))=S_{y}(s c b l)-S_{x}(s s b l) .
$$

We now define the variable the known variable $\alpha_{0}$ as:

$$
\alpha_{0}=S_{y}(s c b l)-S_{x}(s s b l)
$$

and we can write:

$$
p_{\nu}=\frac{\alpha_{0}}{(s c n u)(s s b l)-(s s n u)(s c b l)} .
$$

Similarly, we can solve for $p_{b_{l e p}}$, which will be useful later:

$$
p_{b_{l e p}}=\frac{S_{y}(\operatorname{scnu})-S_{x}(\operatorname{ssnu})}{(\operatorname{scbl})(\operatorname{ssnu})-(\operatorname{ssbl})(\operatorname{scnu})} .
$$

We also write down the following equation for the $\nu$ momentum which again will be useful later:

$$
\begin{array}{r}
p_{\nu_{x}}^{2}+p_{\nu_{y}}^{2}+p_{\nu_{z}}^{2}=p_{\nu}^{2} \\
(\operatorname{scnu})^{2}+(\operatorname{ssnu})^{2}+(\text { cnu })^{2}=1 .
\end{array}
$$


Now we can use the mass of the $W$ and the mass of the top to get the final two equations. First the $W$ mass gives:

$$
\begin{aligned}
m_{W_{l e p}}^{2} & =E_{W_{l e p}}^{2}-\vec{p}_{W_{l e p}}^{2} \\
m_{W_{l e p}}^{2} & =m_{\tau}^{2}+m_{\nu}^{2}+2\left(E_{\tau} E_{\nu}-\overrightarrow{p_{\tau}} \cdot \vec{p}_{\nu}\right) \\
\frac{m_{W_{l e p}}^{2}}{2} & =E_{\tau} E_{\nu}-\overrightarrow{p_{\tau}} \cdot \vec{p}_{\nu} \\
\frac{m_{W_{l e p}}^{2}}{2} & =p_{\tau} p_{\nu}-\frac{p_{\tau_{x}} p_{\nu_{x}}+p_{\tau_{y}} p_{\nu_{y}}+p_{\tau_{z}} p_{\nu_{z}}}{p_{\tau} p_{\nu}} p_{\tau} p_{\nu} \\
\frac{m_{W_{l e p}}^{2}}{2 p_{\tau}} & =p_{\nu}(1-(s c t)(\text { scnu })-(\text { sst })(\text { ssnu })-(\text { ct })(\text { cnu }))
\end{aligned}
$$

We now define the known variable variable $\alpha_{1}$ as:

$$
\alpha_{1}=\frac{m_{W_{l e p}}^{2}}{2 \alpha_{0} p_{\tau}}
$$

so we can write:

$$
\frac{\alpha_{1} \alpha_{0}}{p_{\nu}}=1-(s c t)(s c n u)-(s s t)(s s n u)-(c t)(c n u) .
$$

Equations C.30, C.33, C.40, and top quark mass constraint are 4 indepedent equations we use to solve for the 4 unknown variables.

First, we combine Eqns. C.30 and C.40 to remove $p_{\nu}$.

$$
\begin{aligned}
& \frac{\alpha_{1} \alpha_{0}}{\alpha_{0}}=\frac{(1-(s c t)(\text { scnu })-(s s t)(\text { ssnu })-(c t)(\text { cnu }))}{[(s s b l)(s c n u)-(s c b l)(\text { ssnu })]}, \\
& (c t)(c n u)=1-\left[(s c t)+\alpha_{1}(s s b l)\right](s c n u)-\left[(s s t)-\alpha_{1}(s c b l)\right](s s n u), \\
& (\text { cnu })=\frac{1-\beta_{1}(\text { scnu })-\beta_{2}(\text { ssnu })}{(c t)},
\end{aligned}
$$

where we define $\beta_{1}$ and $\beta_{2}$ as:

$$
\begin{aligned}
& \beta_{1}=(s c t)+\alpha_{1}(s s b l), \\
& \beta_{2}=(s s t)-\alpha_{1}(s c b l) .
\end{aligned}
$$

Next, we combine Eqn. C.43 with Eqn. C.33 to eliminate cnu.

$$
(\text { scnu })^{2}+(\text { ssnu })^{2}+\frac{1}{(c t)^{2}}\left[1-\beta_{1}(\text { scnu })-\beta_{2}(\text { ssnu })\right]^{2}=1,
$$




$$
\begin{gathered}
(c t)^{2}(\text { scnu })^{2}+(c t)^{2}(\text { ssnu })^{2}+1-2 \beta_{1}(\text { scnu })-2 \beta_{2}(\text { ssnu })+ \\
2 \beta_{1} \beta_{2}(\text { scnu })(\text { ssnu })+\beta_{1}^{2}(\text { scnu })^{2}+\beta_{2}^{2}(\text { ssnu })^{2}=(c t)^{2}, \\
{\left[(c t)^{2}+\beta_{1}^{2}\right](\text { scnu })^{2}+2 \beta_{1} \beta_{2}(\text { scnu })(\text { ssnu })+\left[(c t)^{2}+\beta_{2}^{2}\right](\text { ssnu })^{2}-} \\
2 \beta_{1}(\text { scnu })-2 \beta_{2}(\text { ssnu })+\left[1-(c t)^{2}\right]=0, \\
a(\text { scnu })^{2}+2 b(\text { scnu })(\text { ssnu })+c(\text { ssnu })^{2}+2 d(\text { scnu })+2 e(\text { ssnu })+f=0,
\end{gathered}
$$

where:

$$
\begin{aligned}
a & =(c t)^{2}+\beta_{1}^{2}, \\
b & =\beta_{1} \beta_{2}, \\
c & =(c t)^{2}+\beta_{2}^{2}, \\
d & =-\beta_{1}, \\
e & =-\beta_{2}, \\
f & =1-(c t)^{2} .
\end{aligned}
$$

Next, we turn to the equation for the top mass.

$$
\begin{aligned}
m_{t}^{2} & =E_{t}^{2}-\vec{p}_{t}^{2} \\
m_{t}^{2} & =m_{W_{l e p}}^{2}+m_{b_{l e p}}^{2}+2\left(E_{W_{l e p}} E_{b_{l e p}}-\vec{p}_{W_{l e p}} \cdot \vec{p}_{b_{l e p}}\right) .
\end{aligned}
$$

Again, we define the variable $M_{l}$ to make things simpler:

$$
M_{l}=\frac{m_{t}^{2}-m_{W_{l e p}}^{2}-m_{b_{l e p}}^{2}}{2} .
$$

Now:

$$
\begin{aligned}
& M_{l}=E_{W_{l e p}} E_{b_{l e p}}-\vec{p}_{W_{l e p}} \cdot \vec{p}_{b_{l e p}}, \\
& M_{l}=\left(E_{\tau}+E_{\nu}\right) E_{b_{l e p}}-\left(\vec{p}_{\tau}+\vec{p}_{\nu}\right) \cdot \vec{p}_{b_{l e p}}, \\
& M_{l}=p_{\tau} E_{b_{l e p}}+p_{\nu} E_{b_{l e p}}-p_{\tau} p_{b_{l e p}} \cos \theta_{b \tau}-p_{b_{l e p}} p_{\nu}\left(\frac{\vec{p}_{b_{l e p}} \cdot \vec{p}_{\nu}}{p_{b_{l e p}} p_{\nu}}\right)
\end{aligned}
$$


Here, we assume that $m_{b_{l e p}}=0$ to further simplify the equation. Once we do this, we can continue:

$$
\begin{aligned}
M_{l} & =p_{\tau} p_{b_{l e p}}\left(1-\cos \theta_{b \tau}\right),+p_{b_{l e p}} p_{\nu}\left(1-\frac{\vec{p}_{b_{l e p}} \cdot \vec{p}_{\nu}}{p_{b_{l e p}} p_{\nu}}\right), \\
\frac{M_{l}}{p_{b_{l e p}}} & =d_{0}+p_{\nu}[1-(\operatorname{scnu})(s c b l)-(s s n u)(s s b l)-(c n u)(c b l)],
\end{aligned}
$$

where $d_{0}$ is defined as:

$$
d_{0}=p_{\tau} p_{b_{l} e p}\left(1-\cos \theta_{b \tau}\right)
$$

We substitute Eqn. C.30 for $p_{\nu}$ and Eqn. C.43 for cnu. We will skip the algebraic manipulations, but this substitution simplifies to:

$$
\frac{M_{l}}{p_{b_{l e p}}}=\frac{\gamma_{0}+\gamma_{1}(\operatorname{scnu})+\gamma_{2}(\operatorname{ssnu})}{[(s c n u)(s s b l)-(\operatorname{ssnu})(s c b l)]}
$$

where:

$$
\begin{aligned}
\gamma_{0} & =\alpha_{0}\left(1-\frac{(c b l)}{(c t)}\right), \\
\gamma_{1} & =\left[d_{0}(s s b l)-\alpha_{0}(s c b l)+\frac{\beta_{1}(c b l)}{(c t)}\right], \\
\gamma_{2} & =\left[-d_{0}(s c b l)-\alpha_{0}(s s b l)+\frac{\beta_{2}(c b l)}{(c t)}\right] .
\end{aligned}
$$

We substitute Eqn. C.31 into Eqn. C.64 to continue:

$$
\frac{-M_{l}(s c b l)(s s n u)+M_{l}(s s b l)(s c n u)}{S_{x}(\operatorname{ssnu})-S_{y}(\operatorname{scnu})}=\frac{\gamma_{0}+\gamma_{1}(s c n u)+\gamma_{2}(s s n u)}{(\operatorname{scnu})(s s b l)-(\operatorname{ssnu})(s c b l)} \text {. }
$$

Gathering like terms gives us:

$$
A(\operatorname{scnu})^{2}+2 B(\operatorname{scnu})(\operatorname{ssn} u)+C(\operatorname{ssnu})^{2}+2 D_{2}(\operatorname{scnu})+2 E(\operatorname{ssnu})=0,
$$

where:

$$
\begin{aligned}
A & =M_{l}(s s b l)^{2}+\gamma_{1} S_{y} \\
B & =-M_{l}(s s b l)(s c b l)-\frac{1}{2} \gamma_{1} S_{x}+\frac{1}{2} \gamma_{2} S_{y}, \\
C & =M_{l}(s c b l)^{2}-\gamma_{2} S_{x}, \\
D_{2} & =2\left(\frac{1}{2} \gamma_{0} S_{y}\right), \\
E & =2\left(-\frac{1}{2} \gamma_{0} S_{x}\right) .
\end{aligned}
$$


We define the variable $c d i v=c / C$ and multiply Eqn. C.69 by it. Adding this to Eqn. C.48 gives:

$$
a^{\prime}(\text { scnu })^{2}+2 b^{\prime}(\text { scnu })(\text { ssnu })+2 d^{\prime}(\text { scnu })+2 e^{\prime}(\text { ssnu })+f=0
$$

where:

$$
\begin{aligned}
a^{\prime} & =a-(c d i v) A \\
b^{\prime} & =b-(c d i v) B \\
d^{\prime} & =d-(\text { cdiv }) D_{2} \\
e^{\prime} & =e-(\text { cdiv }) E
\end{aligned}
$$

Solving Eqn. C.75 for ssnu gives:

$$
\text { ssnu }=\frac{-f-a^{\prime}(\operatorname{scnu})^{2}-2 d^{\prime}(\operatorname{scn} u)}{2 b^{\prime}(\operatorname{scn} u)+2 e^{\prime}} .
$$

We use this in Eqn. C.48 to solve for scnu. First, we square ssnu:

$$
(\text { ssnu })^{2}=\frac{a^{\prime 2}(\text { scnu })^{4}+G_{3}(\text { scnu })^{3}+G_{2}(\text { scnu })^{2}+G_{1}(\text { scnu })+f^{2}}{4\left[b^{\prime}(\text { scnu })+e^{\prime}\right]^{2}},
$$

where:

$$
\begin{aligned}
& G_{3}=4 d^{\prime} a^{\prime}, \\
& G_{2}=2 a^{\prime} f+4 d^{\prime 2}, \\
& G_{1}=4 d^{\prime} f .
\end{aligned}
$$

Also, we rewrite ssnu with the same denominator as Eqn. C.82 to make the calculation easier.

$$
\begin{aligned}
\text { ssnu } & =\frac{-a^{\prime}(\text { scnu })^{2}-2 d^{\prime}(\operatorname{scn} u)-f}{2\left[b^{\prime}(\operatorname{scn} u)+e^{\prime}\right]} \times \frac{b^{\prime}(\text { scnu })+e^{\prime}}{b^{\prime}(\operatorname{scn} u)+e^{\prime}} \\
\text { ssnu } & =\frac{\Lambda_{3}(\operatorname{scn} u)^{3}+\Lambda_{2}(\text { scnu })^{2}+\Lambda_{1}(\text { scnu })+\Lambda_{0}}{2\left[b^{\prime}(\operatorname{scn} u)+e^{\prime}\right]^{2}}
\end{aligned}
$$


where

$$
\begin{aligned}
& \Lambda_{3}=-a^{\prime} b^{\prime}, \\
& \Lambda_{2}=-a^{\prime} e^{\prime}-2 b^{\prime} d^{\prime}, \\
& \Lambda_{1}=-2 d^{\prime} e^{\prime}-f b^{\prime}, \\
& \Lambda_{0}=-f e^{\prime} .
\end{aligned}
$$

Now that we have defined ssnu and $(\operatorname{ssnu})^{2}$ in terms of scnu, we simply plug them back into Eqn. C.48 and collect like terms:

$$
\begin{gathered}
c(\text { ssnu })^{2}+2 b(\text { scnu })(\text { ssnu })+2 e(\text { ssnu })=-f-a(\operatorname{scnu})^{2}-2 d(\operatorname{scnu}), \\
\beta_{4}(\text { scnu })+\beta_{3}(\text { scnu })+\beta_{2}(\text { scnu })+\beta_{1}(\text { scnu })+\beta_{0}=0,
\end{gathered}
$$

where:

$$
\begin{aligned}
& \beta_{4}=a^{\prime 2} c+4 a b^{\prime 2}+4 b \Lambda_{3}, \\
& \beta_{3}=8 a b^{\prime} e^{\prime}+8 d b^{2}+c G_{3}+4 b \Lambda_{2}+4 e \Lambda_{3}, \\
& \beta_{2}=4 a e^{\prime 2}+16 d b^{\prime} e^{\prime}+4 b^{\prime 2} f+c G_{2}+4 b^{\prime} \Lambda_{1}+4 e \Lambda_{2}, \\
& \beta_{1}=8 d e^{\prime 2}+8 b^{\prime} e^{\prime} f+4 e \Lambda_{1}+4 b \Lambda_{0}+c G_{1}, \\
& \beta_{0}=c f^{2}+4 e \Lambda_{0}+4 f e^{\prime 2} .
\end{aligned}
$$

Solving for the four roots of Eqn. C.93 gives 4 possible solutions for scnu. We can substitute these solutions into previous equations now to obtain ssnu, cnu, and then more importantly $p_{\nu}$ and $p_{b_{l e p}}$.

We impose a few requirements to ensure only good solutions are used. First, we reject any solution which gives the same leptonic $b$ quark momentum as a previous solution. Next, we sum the squares of scnu, ssnu, and cnu and require the sum to be within the range $0.99-1.01$. Finally, we reject any solution with a negative leptonic $b$ momentum or with a neutrino momentum greater than $800 \mathrm{GeV}$. 


\section{Appendix D Validating Transfer Function Fits on New Selection}

It should be noted that the parameters used in building the transfer functions were fit from older MC events which were selected for $e$ or $\mu+$ jets events [53] [93]. We have since added new MC samples with higher instantaneous luminosity to better match the luminosity profile in the more recent data, and we are dealing explicitly with selected $\tau+$ jets events. We do not expect the existence of $\tau$ 's in the selected events, the use of the NN to veto QCD multijet events, or the new luminosity profile to change the shape of the transfer function, but we need to confirm this. To check the energy transfer functions, we check the distribution of $\delta$ (defined as $E_{p r t}-E_{\text {jet }}$ where $E_{\text {prt }}$ and $E_{\text {jet }}$ are the parton and jet energies, respectively) for jets in $\mathrm{MC}$ events with all 4 jets well matched to partons. We do this for light quark and $b$ quark partons separately in the original MC used to fit the TF's, the higher luminosity $\mathrm{MC}, \tau \mathrm{MC}$ events before the $\mathrm{NN}$ selection requirement, and $\tau \mathrm{MC}$ events after the $\mathrm{NN}$ selection requirement. As Fig. D.1 shows, the distributions look the same for all 4 samples, so we can use the previously fit parameters from [93]. For the angular transfer functions, using the same events, we check the distribution of $\theta_{p 1 p 2}-\theta_{j 1 j 2}$, where $\theta_{p 1 p 2}$ refers to the angle between the two partons from the $W$ decay and $\theta_{j 1 j 2}$ refers to the angle between the two jets matched to those partons. Again, we show this distribution for the original MC, the high luminosity $\mathrm{MC}, \tau$ selected $\mathrm{MC}$ events without the $\mathrm{NN}$ selection requirement, and $\tau$ selected MC after the NN selection requirement. We do the same for the distribution of $\theta_{p W p b}-\theta_{j W j b}$ where now the angle refers to the angle between the hadronic side $W$ and $b$ quark. As Fig. D.2 shows, these distributions also look the same for all 4 samples, so we can safely use the previously fit parameters. 

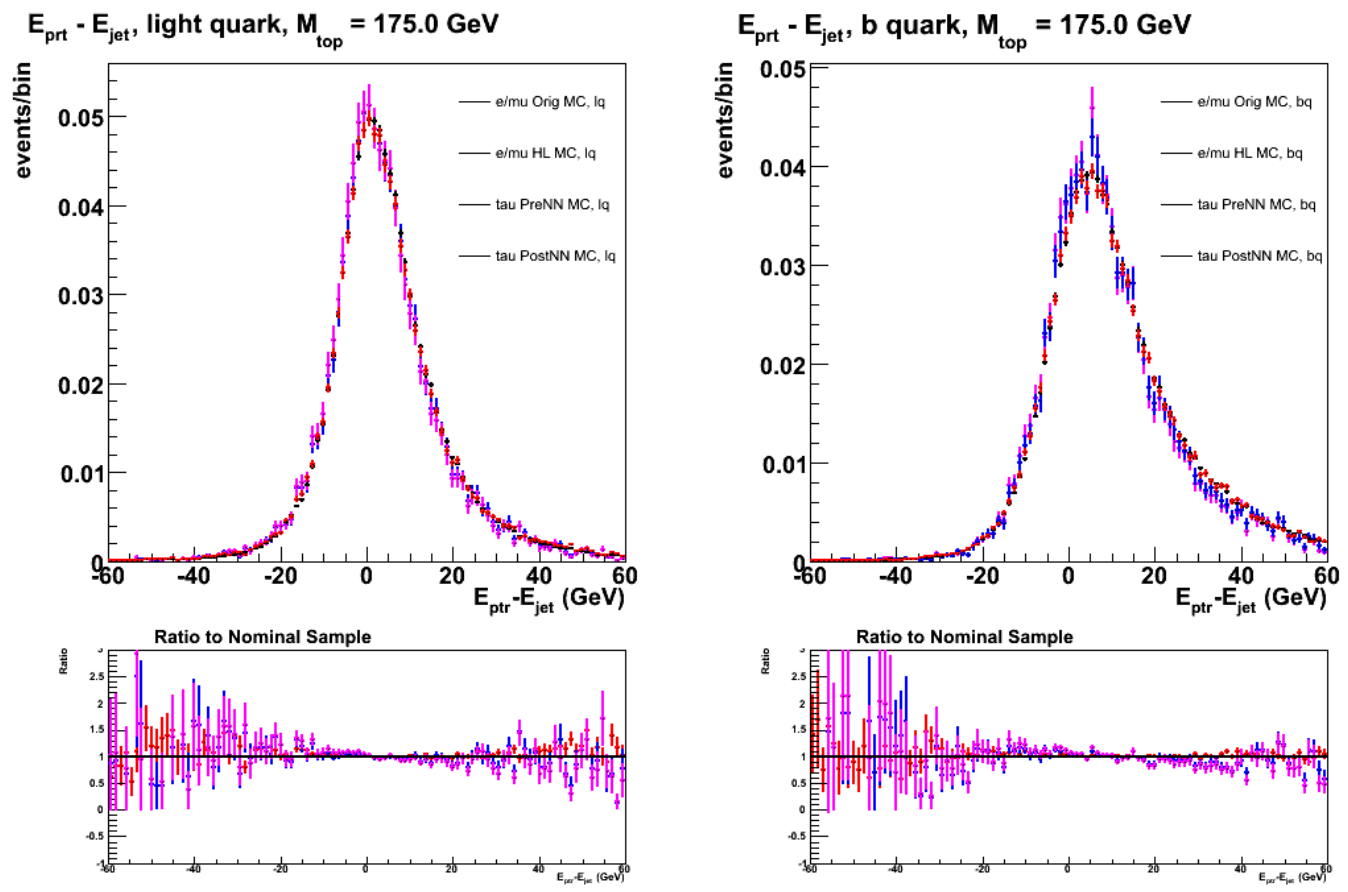

Figure D.1: Energy transfer function distribution for light quark jets (left) and $b$ quark jets (right) for the original MC, high luminosity MC, and $\tau$ events before and after the NN selection requirements are applied. The distributions on the bottom show the ratio of the distribution in the original $\mathrm{MC}$ used to fit the $\mathrm{TF}$ parameters to the distribution in the other three MC samples. 

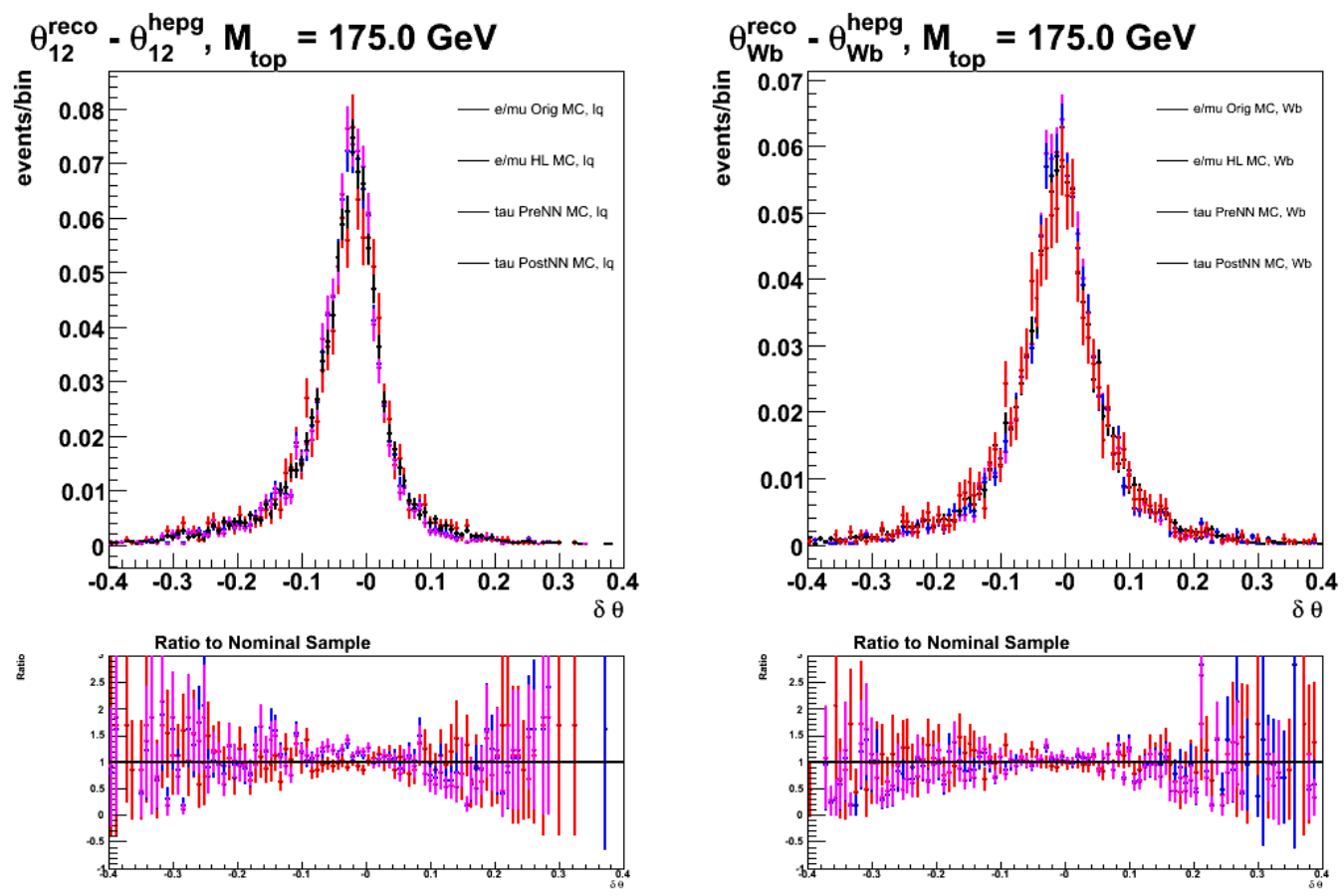

Figure D.2: Angle transfer function distribution for the two jets from the $W$ (left) and the $W$ and $b$ from the hadronically decaying top quark (right) for the original MC, high luminosity $\mathrm{MC}$, and $\tau$ events before and after the NN selection requirements are applied. The distributions on the bottom show the ratio of the distribution in the original MC used to fit the TF parameters to the distribution in the other three MC samples. 


\section{Appendix E}

\section{Parton Showering Systematic on $\sigma_{t \bar{t}}$}

The parton showering systematic on the $\sigma_{t \bar{t}}$ measurement is usually taken to be the difference in the $\sigma_{t \bar{t}}$ as measured using $t \bar{t}$ MC generated with PyTHIA and HeRWIG as described in Sec. 5.4.1. For most analyses measuring the $\sigma_{t \bar{t}}$, this systematic uncertainty is usually in the range of $1-3 \%$ (for example, see [88]). In the $t \bar{t} \rightarrow \tau+$ jets events, we measured a $20.5 \%$ difference between the Pythia and Herwig result.

To verify that the effect is correctly measured, we check the acceptance for $\tau+$ jets events in Pythia and Herwig. Pythia gives an acceptance of $0.121 \%$ while Herwig gives an acceptance of $0.149 \%$ which is a $23.1 \%$ difference. To determine what is causing this difference, we compare the acceptance in the two MC samples at various stages of the event selection and find that the difference comes in at the $\tau$ identification stage.

Since the two MC generators use the same branching fractions for top, $W$, and $\tau$ decay, the higher acceptance in $\tau$ selection suggests that something in the HerwiG events is faking a $\tau$ signature at a higher rate than in PyтніA. We find that if we require the reconstructed $\tau$ to be matched to a generated $\tau$ in the MC, then the acceptance difference between PythiA and HeRwig drops to just 6\%. This makes it clear that the acceptance difference is coming from a higher $\tau$ fake rate in HERwIG. To determine exactly what is faking $\tau$ 's, we match reconstructed $\tau$ 's in PyTHIA and HeRwig to one of the 6 daughters from the $t \bar{t}$ decay. As Fig. E.1 shows, the additional fake $\tau$ 's in Herwig are coming from light quark jets.

Now, we ask the queston, "why do Herwig jets fake $\tau$ 's more often than PythiA jets?" To do this, we apply the $\tau$ selection requirements sequentially and track how many candidates are left after each requirement. Not surprisingly, the requirements giving the largest differences in acceptance are the jet rejection requirements on the 


\section{Tau Matching Test}

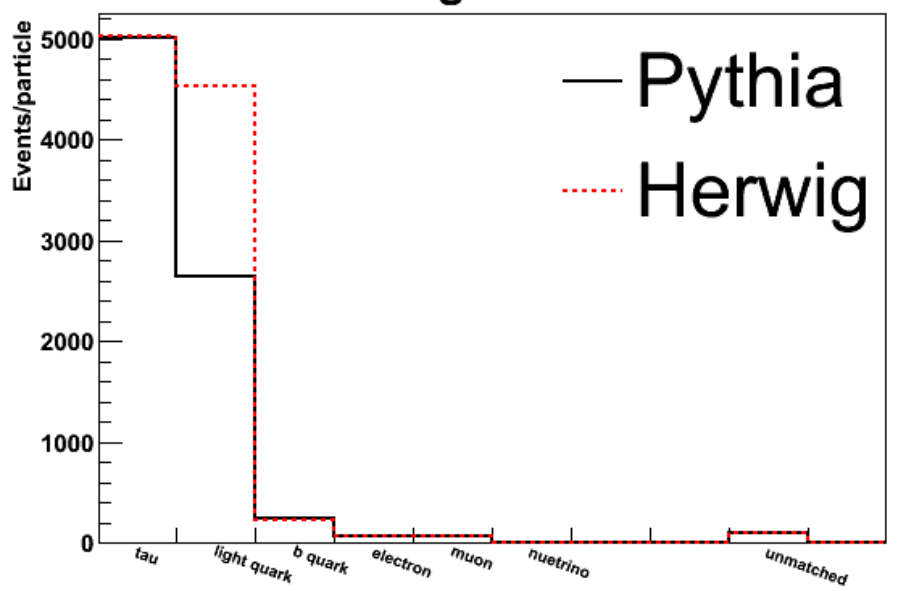

Figure E.1: Distribution showing which of $t \bar{t}$ daughter particles is creating the reconstructed $\tau$ in Pythia and Herwig. The Herwig distribution is scaled so that that first bin will match the result in PYTHIA.

visible mass, the number of tracks in the isolation cone, the calorimeter isolation, and the number of tracks in the signal cone. The visible mass and tracks in isolation annulus requirements are passing more HERWIG $\tau$ 's than PYTHIA. The calorimeter isolation and signal track requirement work in the opposite direction, but they cannot reject all the jets faking $\tau$ 's that are passing the first two requirements. To check this effect, we make distributions of these four variables with all other selection requirements applied for all $\tau$ 's and $\tau$ 's which are matched to generated light quarks. The results can be seen in Figs. E.2 and E.3. As the distributions show, HeRwig jets tend to give more $\tau$ 's with lower visible mass and no tracks in the isolation annulus while PYTHIA jets tend to give more isolated $\tau$ 's with 1 or 3 signal tracks.

In an effort to understand why HERWIG jets give fake $\tau$ 's with different distributions in these variables than PYTHIA jets, we check several other $\tau$ variables for $\tau$ 's matched to generated $\tau$ 's and generated light quarks in both $\mathrm{MC}$ samples. We find the largest difference to be in the number of $\pi^{0}$ s associated with the $\tau$ and the visible mass of the $\tau$. As can be seen in Fig. E.4. HeRwig jets fakes $\tau$ 's with fewer $\pi^{0} s$ than PYTHIA jets. Additionally, the Herwig $\tau$ fakes also have lower visible mass. As visible mass is defined as the mass of the tracks and $\pi^{0} s$ associated with the $\tau$, this variable is directly 

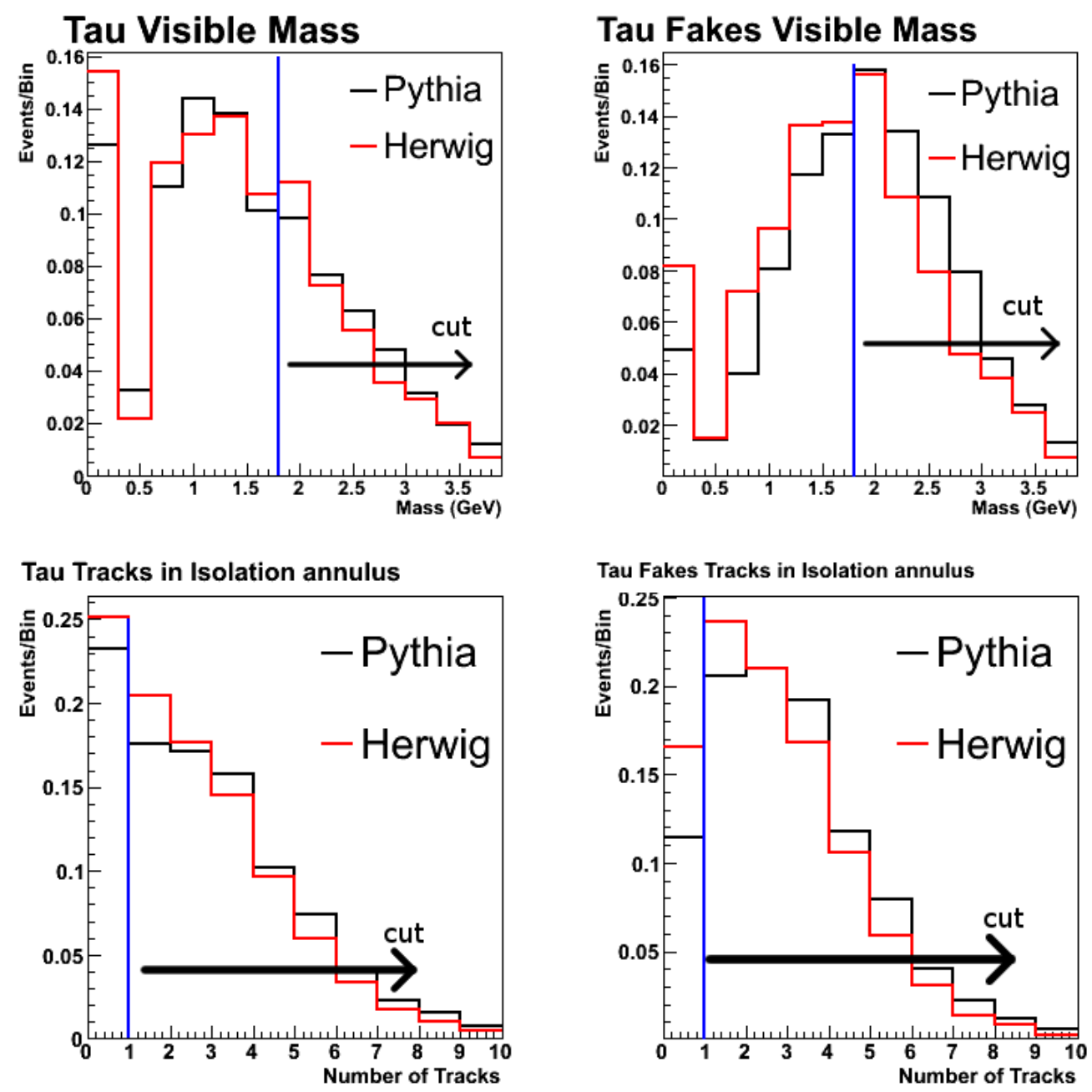

Figure E.2: N - 1 distributions for the visible mass (top) and tracks in isolation annulus (bottom) requirements. On the left, the distribution is made for all $\tau$ 's, and on the right these variables are shown for $\tau$ 's which are matched to generated light quarks. In the case of the light quarks faking $\tau$ 's, HERWIG jets give more $\tau$ 's with lower visible mass and no tracks in the isolation annulus than PYTHIA jets. 

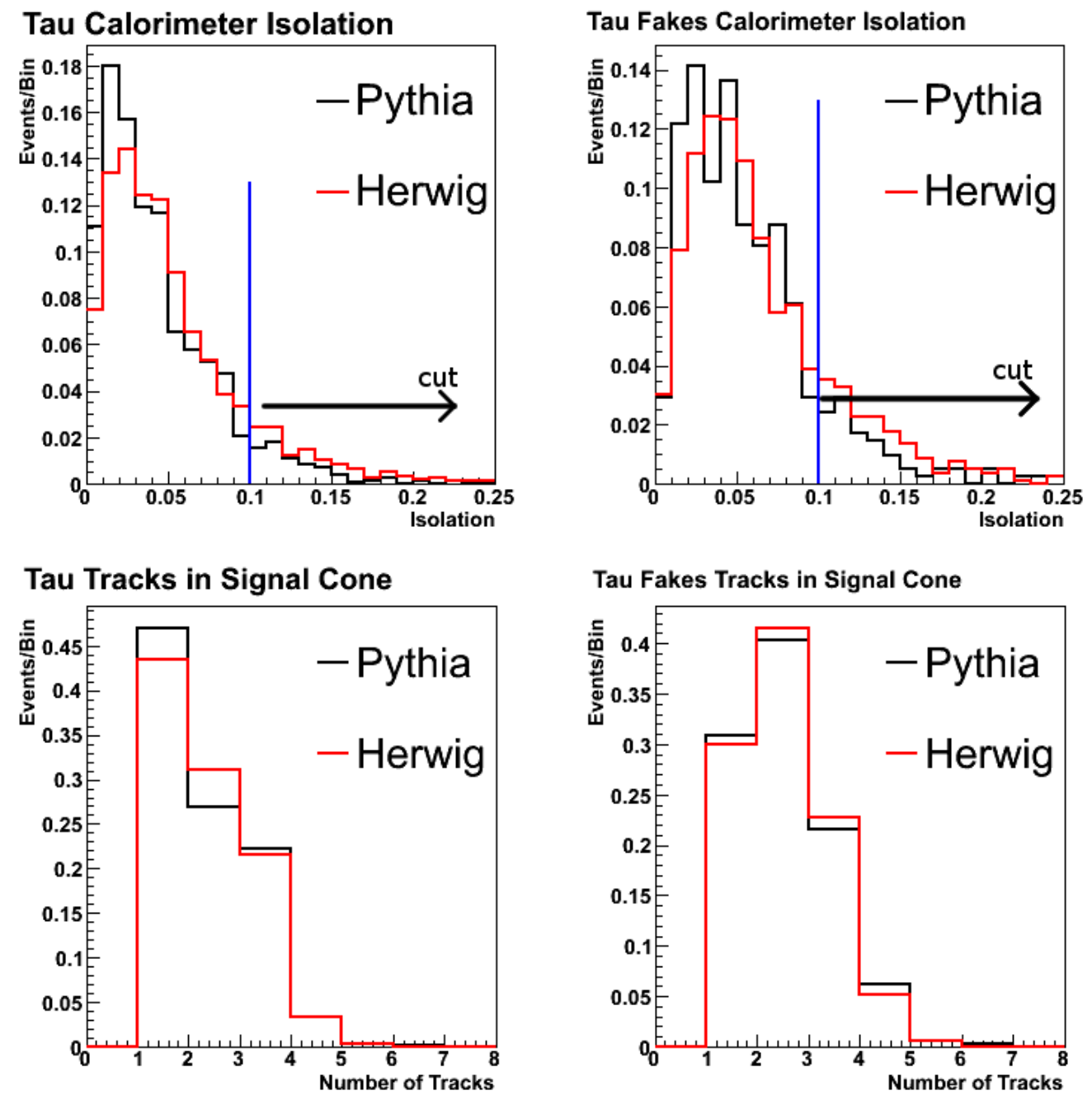

Figure E.3: N - 1 distributions for the calorimeter isolation (top) and tracks in signal cone (bottom) requirements. On the left, the distribution is made for all $\tau$ 's, and on the right these variables are shown for $\tau$ 's which are matched to generated light quarks. In the case of the light quarks faking $\tau$ 's, HERwiG jets are less isolated. 
related to the number of $\pi^{0} s$.

\section{Tau Matched Taus}

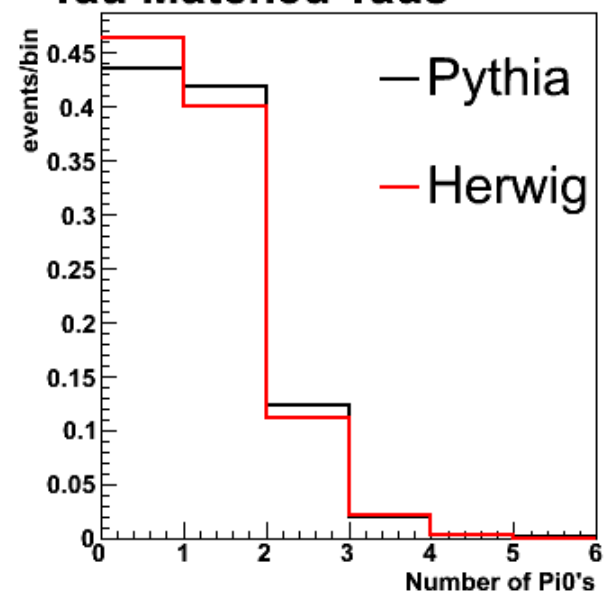

Tau Matched Taus

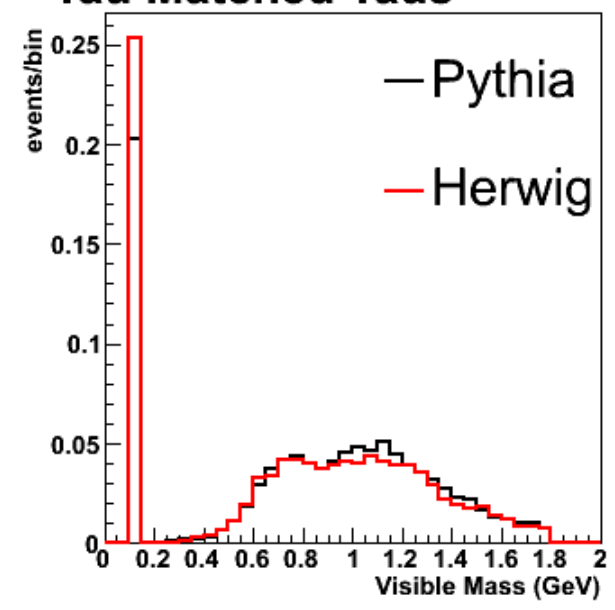

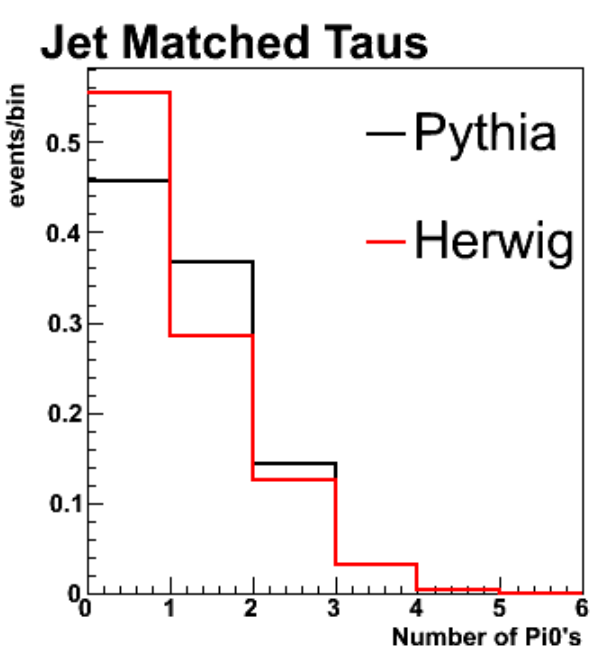

Jet Matched Taus

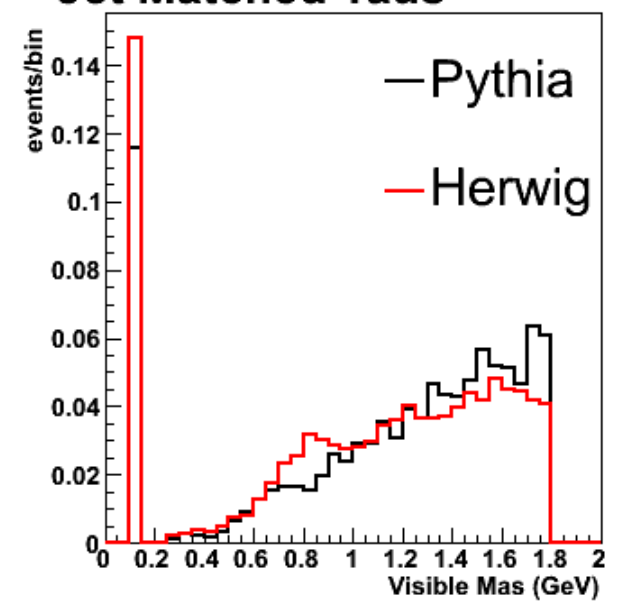

Figure E.4: Number of $\pi^{0}$ 's (top) and visible mass (bottom) for reconstructed $\tau$ 's matched to generated $\tau$ 's (left) and generated light quarks (right) from PYTHIA and HERWIG.

We check the jets from PythiA and Herwig to see if we can discover why these jets give the observed $\tau$ distributions. We find that HERWIG jets tend to have lower EM fraction and track multiplicity than PYTHIA jets. The lower EM fraction in HeRwig jets explains the fewer identified $\pi^{0} s$ and lower visible mass in $\tau$ fakes than from PyтнiA jets. This allows more fake $\tau$ 's from HERWIG jets to pass the $\tau$ selection requirements. Likewise, the lower track multiplicity in HERWIG jets causes the tracks in isolation annulus requirement to remove fewer fake $\tau$ 's than in PYTHIA. 
We discovered that there is significant disagreement in the ratio of charged and neutral particles in the jets with low $P_{T}$ between Pythia and Herwig [102. Both generators are tuned to match LEP data well, but the discrepancy has not been tuned for hadron colliders. To determine which model better matches the $\tau$ fake rate in CDF data, we compare $\tau$ variables in events from the QCD multijets enriched data sample (data selected without the $E_{\mathrm{T}}, \mathrm{NN}$, or b-tagging requirement), the QCD multijets model, and Pythia and Herwig MC in which the $\tau$ has been matched to a light quark jet. Distributions for the number of $\pi^{0}$ 's and the visible mass of the $\tau$ are shown in Fig. E.5. As we expect, the QCD multijets enriched data and the QCD model agree well in both cases which provides a good cross check for the study. We find that the PYTHIA MC generator does a better job of modeling the observed $\tau$ fakes than HeRwiG. This is particularly striking for the visible mass where the HERwIG MC generator gives a significantly different shape than that observed in data, QCD multijets, or PYTHIA.

With the evidence showing that the PyтнIA MC generator models the fake rate in data better than the HeRwig model, we reweight HeRwig events in which the $\tau$ is matched to a light quark jet by the visible mass distribution from Fig. E.2. Doing this gives us Herwig events in which the $\tau$ fake rate is closer to that observed in PythiA. After reweighting, we again check the acceptance and measure the cross section with events from Pythia and the reweighted Herwig. The results are given in Tab. E.1. After reweighting, we find that the difference between the two MC generators to be $\sim 3 \%$.

Since we have shown that the $\tau$ fake rate in HERWIG is higher than that observed in the data, it does not seem necessary to take a $20 \%$ systematc uncertainty on the parton showering generator. We instead assign a $6 \%$ uncertainty on the $\mathrm{MC}$ generator. This matches the acceptance difference observed in PYTHIA and HERWIG after requiring that the reconstructed $\tau$ be matched to a generated $\tau$. It is also a conservative estimate of the uncertainty since $6 \%$ is roughly twice the difference between the PYTHIA and the reweighted HERwIG results and twice the difference generally seen in electron or muon + jets analyses. 


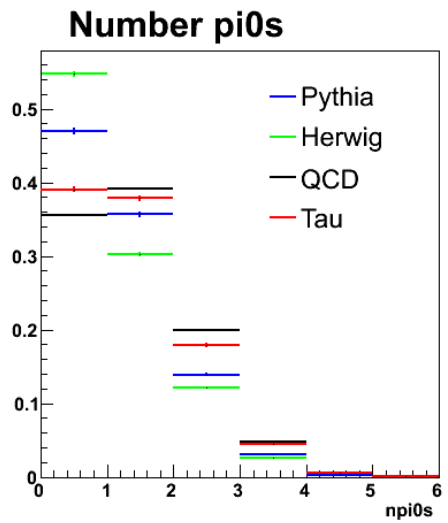

Visible mass

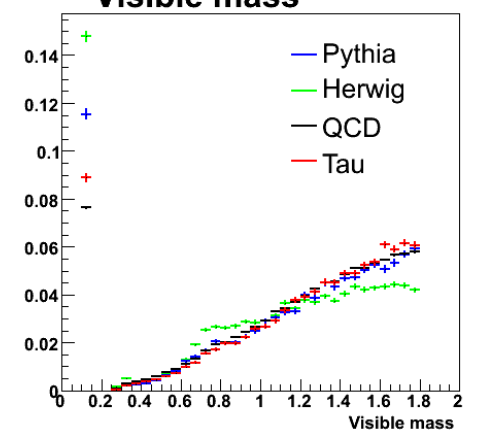

Data/MC Comparison

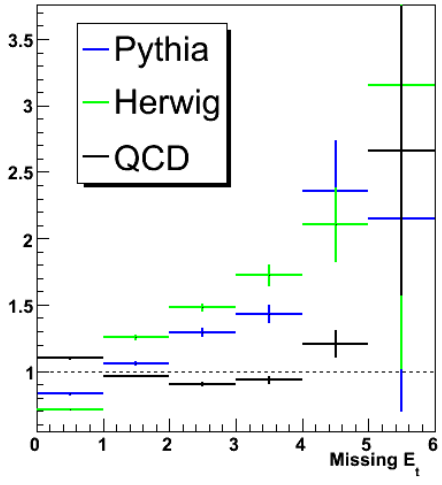

Data/MC Comparison

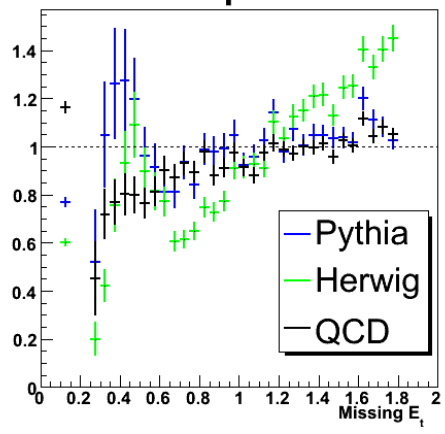

Figure E.5: Distributions of variables from $\tau$ 's matched to light quark jets from PYTHIA, HERWIG, QCD multijets and data without the $E_{\mathrm{T}}, \mathrm{NN}$, and b-tagging requirements applied. On the left, the normalized distribution is shown for Pythia, Herwig, QCD multijets, and data jets. On the right, the ratio of QCD/Data, PYTHIA/Data and HeRwig/Data is shown for each bin. The top distribution is for the number of $\pi^{0}$ 's and the bottom is for the visible mass.

\begin{tabular}{|l||c|c|}
\hline Pretag & Acc (\%) & \% Difference \\
\hline Pythia & 0.1885 & \\
Herwig & 0.2350 & 24.7 \\
Herwig Reweighted & 0.1961 & 4.0 \\
\hline \hline Tag & Acc (\%) & $\%$ Difference \\
\hline Pythia & 0.1209 & \\
Herwig & 0.1494 & 23.6 \\
Herwig Reweighted & .1247 & 3.1 \\
\hline \hline Final & $\sigma_{t \bar{t}}(\mathrm{pb})$ & $\%$ Difference \\
\hline Pythia & 8.8 & \\
Herwig & 7.0 & 20.5 \\
Herwig Reweighted & 8.5 & 3.4 \\
\hline
\end{tabular}

Table E.1: Acceptance and $\sigma_{t \bar{t}}$ measured from Pythia, Herwig, and reweighted HeRWIG. The reweighting brings the difference down from $\sim 20 \%$ to $\sim 3 \%$. 


\section{Appendix F}

\section{The Bootstrapping Method}

A proper way to measure the uncertainty on the mean value of a measurement is to gather multiple datasets from the parent distribution, and measure the mean of each dataset. We then extract the uncertainty on a given measured mean from the distribution of the means. However, in some cases, such as this one, this would be an extremely time consuming process. Instead, we use a statistical procedure called bootstrapping to simulate the creation of multiple datasets[96] [103]. The idea behind the bootstrapping method is to use the single dataset we have to create multiple datasets all representative of random selection from the same parent distribution. We then use the spread of the means of all the new datasets to measure the uncertainty on the mean. We begin with a single set of $\mathrm{N}$ events, and we assume this set of events is a good representation of the parent distribution of all such events. This is a safe assumption as long as $\mathrm{N}$ is large enough. We then create $\mathrm{P}$ new datasets named $X_{1}, X_{2}, \ldots, X_{P}$ each with $\mathrm{N}$ events by randomly sampling events with replacement from the original set of $\mathrm{N}$ events. Although each of the $\mathrm{X}$ datasets will have repeated events within them, if the original set of $\mathrm{N}$ events is a good representation of the full parent distribution, then each of the $\mathrm{X}$ datasets randomly selected from these events should be a good approximation of an independent set of $\mathrm{N}$ events selected from the parent distribution. We have thus created $\mathrm{P}$ datasets each with $\mathrm{N}$ events without having to generate more events. We carry out the measurement of $M_{t o p}$ on each sample, and consider the distribution of the resulting means $\mu_{1}, \mu_{2}, \ldots, \mu_{P}$. We fit this distribution with a Gaussian function and take the width of the Gaussian to be the uncertainty on the measurement of the mean. See Fig. F.1 for a graphical representation of this process.

To clarify this procedure, we provide an example of the bootstrapping method with 
a toy Gaussian model in Fig. F.2. In this model, we wish to measure a variable $\mathrm{X}$ which comes from a normal distribution (Fig. F.2a). To estimate the mean value of $\mathrm{X}$ $(\langle X\rangle)$, we measure $\mathrm{X}$ across an ensemble of 1000 event PEs. However, for the sake of this model, assume we only have 20000 unique events to use in the measurement. The distribution of $\mathrm{X}$ in these 20000 events is displayed in Fig. F.2b along with the shape of the parent distribution to guide to the eye. The problem is that without resampling events, we are only able to create 20 unique PE's with these events. As can be seen in Fig. F.2c we can not estimate the distribution of $\mathrm{X}$ very well from only 10 PE's. As a result, we choose to resample the events from the 20000 original unique events by a factor of 20 which allows us to create $400 \mathrm{PE}$ 's. With 400 measurement of $\langle X\rangle$, shown in Fig. F.2d, we now fit a Gaussian to measure the mean value of $\mathrm{X}$. In this model, we measure $\langle X\rangle$ to be -0.002 . We measure the standard deviation from the 400 PEs to be 0.032. Since we used 400 PE's to calculate this, we would estimate the uncertainty on the mean to be $0.032 / \sqrt{400}=0.002$. However, we do not really have enough events to form 400 unique PE's, so this method underestimates the uncertainty on the measurement of the mean. To correctly measure the uncertainty on the mean, we use the bootstrapping method. We use the original 20000 unique events to create 500 new samples with 20000 events by randomly sampling with replacement from the unique events. Two of these 500 new distributions can be seen in Figs. F.2e and F.2f. They are displayed along with the parent distribution scaled to the maximum bin to show that these bootstrapped samples are decent estimations of the parent distribution. Even though these distributions are derived from the same events, they are all statistically different. We measure $\langle X\rangle$ in each of these bootstrapped samples by creating ensembles of 1000 event PE's along with the same resampling factor of 20 we used before. We then fit a Gaussian to the distribution of means measured from the 500 bootstrapped samples. The standard deviation of this distribution shown in Fig. F.2g] is taken to be the uncertainty on the measurement of $\langle X\rangle$. With this model, we estimate $\langle X\rangle$ to be $-0.002 \pm 0.007$. This is in good agreement with 0 , the true mean value of $\mathrm{X}$ from the parent distribution. 


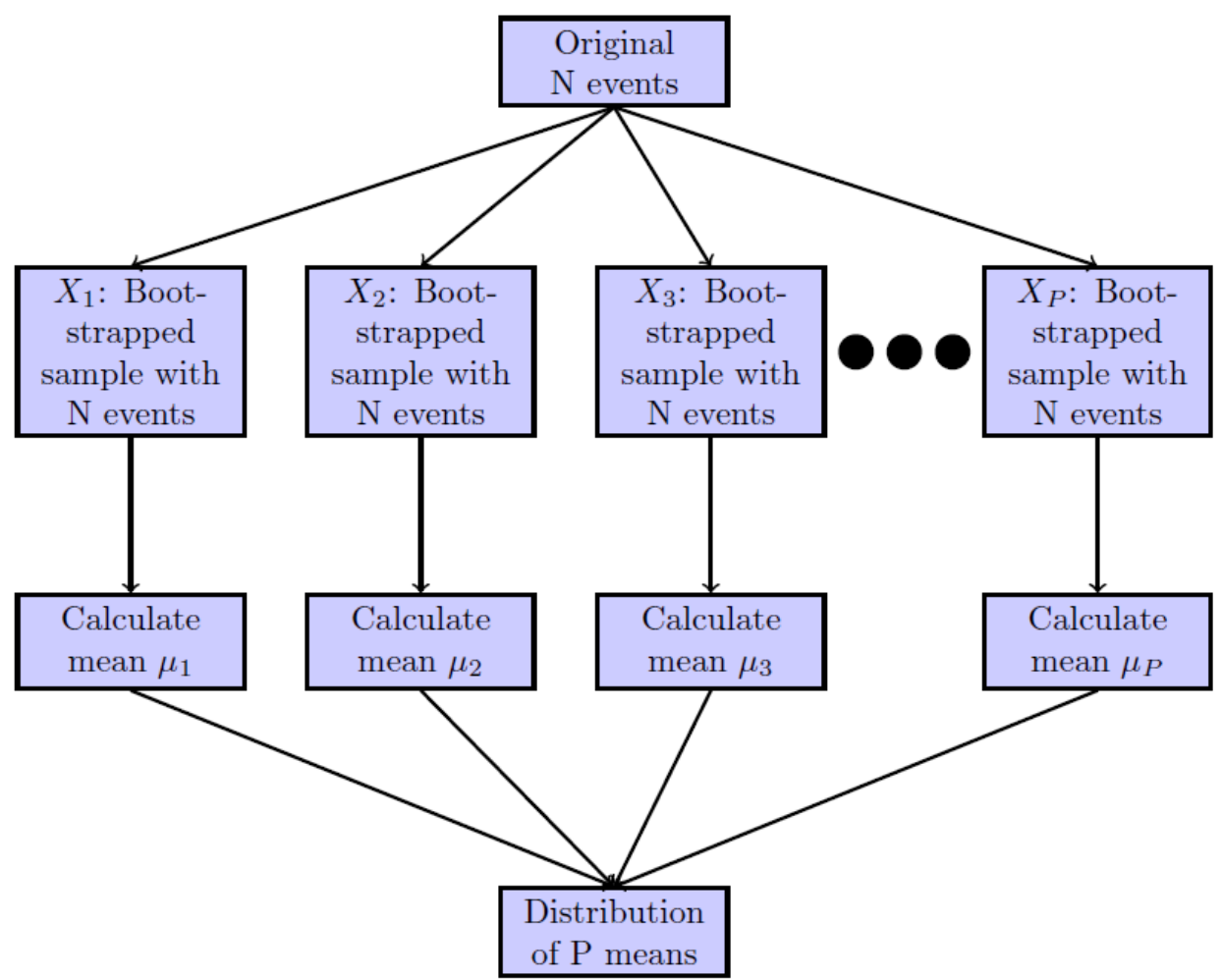

Figure F.1: Diagram explaining the bootstrapping method of error estimation. 


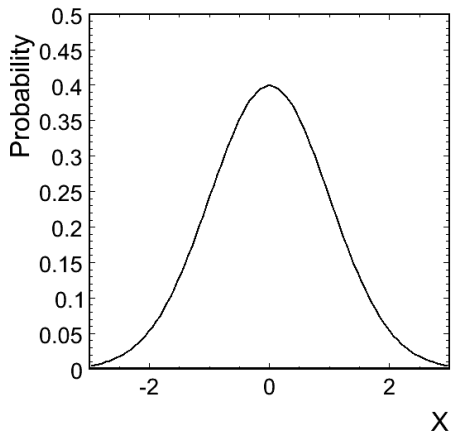

(a)

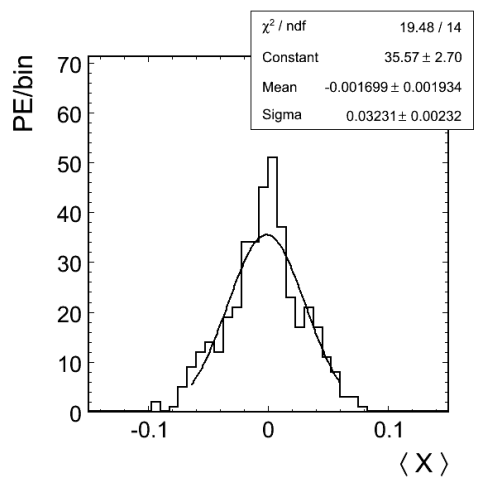

(d)

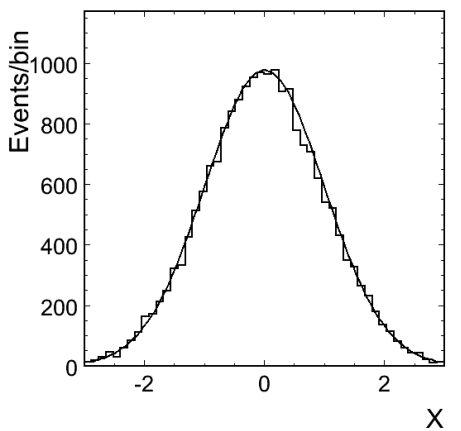

(b)

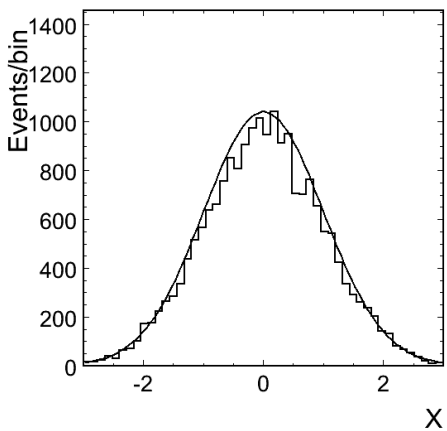

(e)

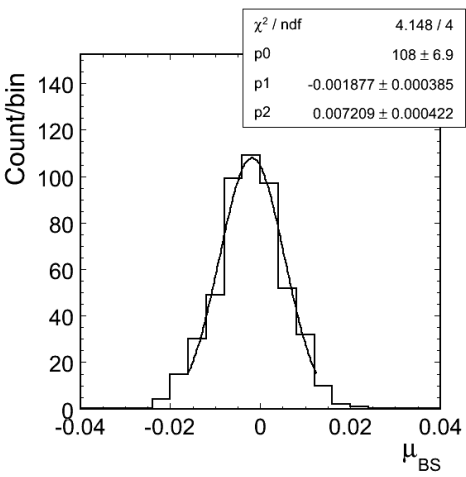

(g)

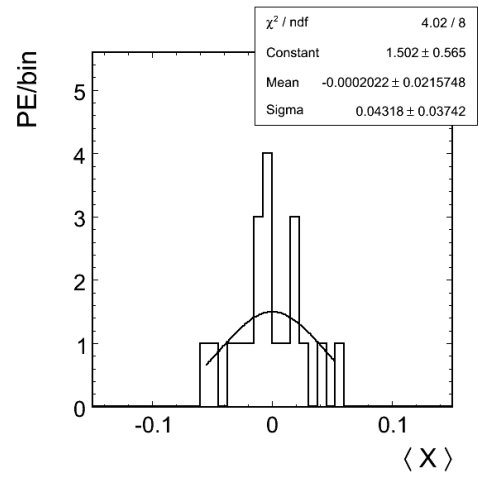

(c)

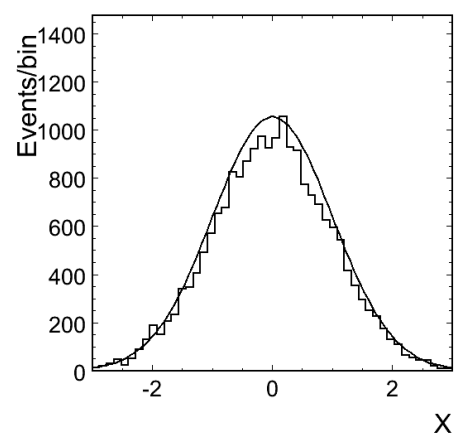

(f)

Figure F.2: Bootstrapping demonstration with Gaussian toy model. We start with the parent distribution of $\mathrm{X}$ in Fig. (a) and the 20000 unique events selected from the parent distribution in Fig. (b). Figures (c) and (d) show the measurements of the mean value of $\mathrm{X}$ with 1000 event PE's without and with using resampling. We take the mean value of -0.002 from the measurement with resampling as the measured mean value of X. Figures (e) and (f) show 2 of the 500 bootstrapped samples used to help determine the uncertainty on the mean measurement. Finally, Fig. (g) shows the distribution of the measured mean values of $\mathrm{X}$ from each bootstrapped sample. The standard deviation of this distribution, 0.007 , is the uncertainty on the measured mean of -0.002 . In this toy model, we measure the mean value of $\mathrm{X}$ to be $-0.002 \pm 0.007$ which agrees well with 0 , the known mean of the normal distribution. 


\section{References}

[1] V. Abazov et al., "Measurement of ttbar production in the tau + jets topology using ppbar collisions at $\sqrt{s}=1.96 \mathrm{TeV}, "$ Phys. Rev., vol. D82, p. 071102, 2010.

[2] J. A. Coarasa Perez, J. Guasch, and J. Sola, "Top quark and charged Higgs at the Tevatron Run II," 1999.

[3] K. Nakamura et al., "Particle Data Group," J. Phys. G, vol. 37, no. 075021, 2010.

[4] J. W. F. Valle, "Neutrino physics overview," J. Phys. Conf. Ser., vol. 53, pp. 473$505,2006$.

[5] D. Griffiths, Introduction to Elementary Particles. John Wiley \& Sons, Inc., 1987.

[6] F. Halzen and A. Martin, Quarks and Leptons. John Wiley \& Sons, Inc., 1984.

[7] M. E. Peskin and D. V. Schroeder, An Introduction to Quantum Field Theory. Westview Press, 1995.

[8] I. Aitchison and A. Hey, Gauge Theory in Particle Physics, Volume I and II. Taylor and Francis, 2003.

[9] K. Nakamura et al., "Review of Particle Physics: The CKM Quark-Mixing Matrix," Journal of Phys. G, vol. 37, 2010. http://pdg.lbl.gov/2010/reviews/ rpp2010-rev-ckm-matrix.pdf.

[10] R. Barate et al., "Search for the standard model Higgs boson at LEP," Phys. Lett., vol. B565, pp. 61-75, 2003.

[11] The CDF and D0 Collaborations, the Tevatron New Phenomena, and Higgs Working Group, "Combined CDF and D0 Upper Limits on Standard Model Higgs Boson Production with up to $8.6 \mathrm{fb}^{-1}$ of Data," 2011. 
[12] The LEP Electroweak Working Group. http://lepewwg.web.cern.ch/ LEPEWWG/.

[13] F. Abe et al., "Observation of Top Quark Production in $\bar{p} p$ Collisions with the Collider Detector at Fermilab," Phys. Rev. Lett., vol. 74, pp. 2626-2631, Apr 1995.

[14] S. Abachi et al., "Search for High Mass Top Quark Production in $p \bar{p}$ Collisions at $\sqrt{s}=1.8 \mathrm{TeV}, "$ Phys. Rev. Lett., vol. 74, pp. 2422-2426, Mar 1995.

[15] C. Campagnari and M. Franklin, "The Discovery of the top quark," Rev. Mod. Phys., vol. 69, pp. 137-212, 1997.

[16] M. Lancaster, "Combination of CDF and DO results on the mass of the top quark using up to $5.8 \mathrm{fb}^{-1}$ of data," 2011.

[17] M. Cacciari, S. Frixione, M. L. Mangano, P. Nason, and G. Ridolfi, "The t anti-t cross-section at $1.8-\mathrm{TeV}$ and $1.96-\mathrm{TeV}$ : A study of the systematics due to parton densities and scale dependence," JHEP, vol. 04, p. 068, 2004.

[18] CDF Collaboration, "Combination of CDF top quark pair production cross section measurements with up to $4.6 \mathrm{fb}^{-1}$." CDF conference note: http://www-cdf . fnal.gov/physics/new/top/confNotes/cdf9913_ttbarxs4invfb.ps.

[19] Tevatron Electroweak Working Group, "Combination of CDF and D0 Measurements of the Single Top Production Cross Section," 2009.

[20] CDF, "CDF Top Quark Physics Public Results: Top Quark Cross Sections." http://www-cdf.fnal.gov/physics/new/top/public_xsection.html

[21] CDF, "CDF Top Quark Physics Public Results: Top Quark Mass." http: //www-cdf.fnal.gov/physics/new/top/public_mass.html.

[22] M. L. Perl et al., "Evidence for anomalous lepton production in $\mathrm{e}^{+} \mathrm{e}^{-}$annihilation," Physical Review Letters, vol. 35, pp. 1489-1492, Dec. 1975. 
[23] T. Aaltonen et al., "Search for charged Higgs bosons in decays of top quarks in $\mathrm{p}$ anti-p collisions at $\sqrt{s}=1.96 \mathrm{TeV}, "$ Phys.Rev.Lett., vol. 103, p. 101803, 2009.

[24] Fermilab, "Accelerator Concepts." http://www-bdnew.fnal.gov/operations/ rookie_books/Concepts_v3.6.pdf.

[25] Fermilab, "Antiproton Source." http://www-bdnew.fnal.gov/operations/ rookie_books/Pbar_v2.2.pdf.

[26] Fermilab, "Accelerator Division Plots." http://www-bd.fnal.gov/pplot/ index.html.

[27] Fermilab, "Tevatron Accelerator Complex." http://www.fnal.gov/pub/today/ images08/00-0635D_hr.jpg.

[28] R. Blair et al., "The CDF-II detector: Technical design report," FERMILABDESIGN-1996-01.

[29] F. Abe et al., "The CDF detector: an overview," Nuclear Instruments and Methods in Physics Research A, vol. 271, no. 3, pp. 387-403, 1988.

[30] Fermilab, "Elevation View of CDF." http://hep-www.px.tsukuba.ac.jp/ $\sim$ hara/isl/cdfii-elev.GIF

[31] T. Akimoto et al., "The CDF Run IIb Silicon Detector: Design, preproduction, and performance," Nuclear Instruments and Methods in Physics Research Section A: Accelerators, Spectrometers, Detectors and Associated Equipment, vol. 556, no. 2 , pp. $459-481,2006$.

[32] W. Ashmanskas et al., "Silicon vertex tracker: a fast precise tracking trigger for CDF," Nuclear Instruments and Methods in Physics Research Section A: Accelerators, Spectrometers, Detectors and Associated Equipment, vol. 447, no. 1-2, pp. $218-222,2000$.

[33] P. Lujan, "An Ace's Guide to the CDF Run II Detector." http: //www-cdfonline.fnal.gov/ops/ace2help/detector_guide/detector_ guide.html. 
[34] C. S. Hill, "Operational experience and performance of the CDFII silicon detector," Nuclear Instruments and Methods in Physics Research Section A: Accelerators, Spectrometers, Detectors and Associated Equipment, vol. 530, no. 1-2, pp. $1-6,2004$. Proceedings of the 6th International Conference on Large Scale Applications and Radiation Hardness of Semiconductor Detectors.

[35] A. Affolder et al., "Intermediate silicon layers detector for the CDF experiment," Nuclear Instruments and Methods in Physics Research Section A: Accelerators, Spectrometers, Detectors and Associated Equipment, vol. 453, no. 1-2, pp. 84 88, 2000. Proc. 7th Int. Conf on Instrumentation for colliding Beam Physics.

[36] T. Affolder et al., "CDF Central Outer Tracker," Nuclear Instruments and Methods in Physics Research Section A: Accelerators, Spectrometers, Detectors and Associated Equipment, vol. 526, no. 3, pp. 249 - 299, 2004.

[37] E. Halkiadakis, "Proceedings for TASI 2009 Summer School on "Physics of the Large and the Small": Introduction to the LHC experiments," 2010.

[38] L. Balka et al., "The CDF central electromagnetic calorimeter," Nuclear Instruments and Methods in Physics Research Section A: Accelerators, Spectrometers, Detectors and Associated Equipment, vol. 267, no. 2-3, pp. 272 - 279, 1988.

[39] G. Apollinari, "Test beam results from the CDF plug upgrade calorimeter," $N u$ clear Instruments and Methods in Physics Research Section A: Accelerators, Spectrometers, Detectors and Associated Equipment, vol. 409, no. 1-3, pp. 547 - 553, 1998.

[40] A. Bhatti et al., "Determination of the jet energy scale at the Collider Detector at Fermilab," Nuclear Instruments and Methods in Physics Research Section A: Accelerators, Spectrometers, Detectors and Associated Equipment, vol. 566, no. 2, pp. $375-412,2006$.

[41] S. Bertolucci et al., "The CDF central and endwall hadron calorimeter," Nuclear Instruments and Methods in Physics Research Section A: Accelerators, Spectrometers, Detectors and Associated Equipment, vol. 267, no. 2-3, pp. 301 - 314, 1988. 
[42] M. Albrow et al., "The CDF plug upgrade electromagnetic calorimeter: test beam results," Nuclear Instruments and Methods in Physics Research Section A: Accelerators, Spectrometers, Detectors and Associated Equipment, vol. 480, no. 2-3, pp. $524-546,2002$.

[43] Fermilab, "Silicon Tracking System." http://www-cdf.fnal.gov/upgrades/ silicon/img6.gif.

[44] A. Artikov et al., "Design and construction of new central and forward muon counters for CDF II," Nuclear Instruments and Methods in Physics Research Section A: Accelerators, Spectrometers, Detectors and Associated Equipment, vol. 538, no. 1-3, pp. $358-371,2005$.

[45] G. Ascoli et al., "CDF central muon detector," Nuclear Instruments and Methods in Physics Research Section A: Accelerators, Spectrometers, Detectors and Associated Equipment, vol. 268, no. 1, pp. 33 - 40, 1988.

[46] T. Dorigo, "The muon system upgrade for the CDF II experiment," Nuclear Instruments and Methods in Physics Research Section A: Accelerators, Spectrometers, Detectors and Associated Equipment, vol. 461, no. 1-3, pp. 560 - 562, 2001. 8th Pisa Meeting on Advanced Detectors.

[47] D. Acosta et al., "The CDF Cherenkov luminosity monitor," Nuclear Instruments and Methods in Physics Research Section A: Accelerators, Spectrometers, Detectors and Associated Equipment, vol. 461, no. 1-3, pp. 540 - 544, 2001. 8th Pisa Meeting on Advanced Detectors.

[48] S. Jindariani et al., "Luminosity Uncertainty for Run 2 up until August 2004." CDF Conference note: CDF/ANAL/CDF/7446.

[49] CDF Collaboration, "Drawings of the CDF Detector." http://www-cdf.fnal. gov/internal/upgrades/detector_drawings.html

[50] V. Sorin, "CDF Trigger System." dept.physics.upenn.edu/ thomsone/ hepseminars/talks/2006_sorin.pdf. 
[51] A. Mitra, S. Wang, and S. Tsai, "Study of Multijet Triggers for all-Hadronic Higgs Search.” CDF Internal note: CDF/ANAL/TRIGGER/CDFR/9954.

[52] G. Compostella, D. Lucchesi, and S. Amerio, "Measurement of the t-tbar production cross section in the MET+jets channel $\left(2.2 \mathrm{fb}^{-1}\right)$." CDF Internal note: CDF/ANAL/TOP/CDFR/9873.

[53] T. Aaltonen et al., "Measurement of the top-quark mass in the lepton+jets channel using a matrix element technique with the CDF II detector," arXiv:1108.1601 hep-ex, Accepted by Phys. Rev. D Rapid Communications, Sept 2011.

[54] T. Sjostrand et al., "High-energy physics event generation with PYTHIA 6.1," Comput. Phys. Commun., vol. 135, pp. 238-259, 2001.

[55] G. Corcella et al., "HERWIG 6.5," JHEP, vol. 0101, 2001.

[56] CDF Jet Energy and Resolution Group, "Generic Jet Energy Corrections at CDF." http://www-cdf.fnal.gov/physics/new/top/2004/jets/cdfpublic. html.

[57] D. Acosta et al., "Measurement of the $t \bar{t}$ production cross section in $p \bar{p}$ collisions at $\sqrt{s}=1.96 \mathrm{TeV}$ using lepton + jets events with secondary vertex $b$-tagging," Phys. Rev. D, vol. 71, p. 052003, Mar 2005.

[58] C. Neu and The CDF Collaboration, "CDF b-tagging: Efficiency and Mistag Measurements," CDF/PUB/SEC_VTX/PUBLIC/8102.

[59] CDF High $P_{T}$ B-Tag Group, "High- $P_{T}$ B-Tagging at CDF-II." http://www-cdf . fnal.gov/physics/new/top/2004/btag/.

[60] CDF High $P_{T}$ B-Tag Group. http://www-cdf.fnal.gov/internal/physics/ top/RunIIBtag/bTag.html.

[61] E. Halkiadakis, C. Hays, M. Tecchio, and W. Yao, "A Conversion Removal Algorithm for the 2003 Winter Conferences," CDF/DOC/TOP/CDFR/6250. 
[62] CDF Collaboration, "CDF Pictures." http://www-cdf.fnal.gov/events/ CDFPictures.html.

[63] Z. Was, "TAUOLA the library for tau lepton decay, and KKMC / KORALB / KORALZ /... status report," Nucl.Phys.Proc.Suppl., vol. 98, pp. 96-102, 2001.

[64] M. Mangano et al., "ALPGEN," J. High. Energy. Phys., vol. 0307, p. 001, 2003.

[65] M. Mangano et al., "Matching matrix elements and shower evolution for top-quark production in hadronic collisions," JHEP, vol. 0701, p. 013, 2007.

[66] Franklin et al., "Heavy-Flavor Content of the W+Jets Sample." CDF Internal note: CDF/ANAL/TOP/CDFR/8765.

[67] "GEANT," Nuclear Instruments and Methods in Physics Research A, vol. 506, no. 3, pp. 250-330, 2003.

[68] "TMultiLayerPerceptron: Designing and using Multi-Layer Perceptrons with ROOT." http://www.fynu.ucl.ac.be/users/c.delaere/MLP/

[69] "Root: A Data Analysis Framework." http://root.cern.ch/drupal/.

[70] "TMultiLayerPerceptron." http://root.cern.ch/root/html/ TMultiLayerPerceptron.html.

[71] J. Bonnans, J. Gilbert, C. Lemaréchal, and C. Sagastizábal, Numerical Optimization. Springer Berlin Heidelberg, 2006.

[72] T. Aaltonen et al., "First Measurement of the Ratio $\sigma_{t-t b a r} / \sigma_{Z / \gamma * \rightarrow l l}$ and Precise Extraction of the t-tbar Cross Section," Phys.Rev.Lett., vol. 105, p. 012001, 2010.

[73] W. Sakumoto, "Event $|Z v t x|<60$ Cut Acceptance for Run II (to Feb06)." CDF Internal note: CDF/ANAL/ELECTROWEAK/CDFR/8318.

[74] CDF Joint Physics Group, "Efficiency of the Z-vertex cut of 60 cm." http://www-cdf.fnal.gov/internal/physics/joint_physics/ instructions/zvertex-efficiency.html. 
[75] Y. Tu and P. Murat, "Identification of the Hadronic Decays of Tau Leptons." CDF Internal note: CDF/DOC/ELECTROWEAK/CDFR/8470.

[76] J. Freeman, S. Grinstein, T. Junk, and E. Palencia, "SecVtx Scale Factors and Mistag Matrices for $2.2 \mathrm{fb}^{-1}$ of Data up to P13." CDF Internal note: CDF/DOC/SEC_VTX/CDFR/9280.

[77] J. Freeman, S. Grinstein, T. Junk, and E. Palencia, "SecVtx Scale Factors and Mistag Matrices for $2.2 \mathrm{fb}^{-1}$ of Data up to P13." CDF Internal note: CDF/DOC/SEC_VTX/CDFR/9280.

[78] S. Budd, T. Junk, T. Liss, and C. Neu, "Tight, Loose and Ultratight SECVTX Tag Rate Matrix with $1.2 \mathrm{fb}^{-1}$." CDF Internal note: CDF/DOC/SEC_VTX/CDFR/8519.

[79] D. Amidei et al., "Calibration of Heavy-Flavor Production in W + 1 Jet Data." CDF Internal note: CDF/ANAL/TOP/CDFR/9187.

[80] A. Ivanov, T. Schwarz, and R. Erbacher, "Calibration of Heavy-Flavor Content in W + Jets Data." CDF Internal note: CDF/ANAL/TOP/CDFR/9403.

[81] S. D. Drell and T.-M. Yan, "Massive Lepton-Pair Production in Hadron-Hadron Collisions at High Energies," Phys. Rev. Lett., vol. 25, pp. 316-320, Aug 1970.

[82] A. Abulencia et al., "Top quark mass measurement using the template method in the lepton + jets channel at CDF II," Phys. Rev. D, vol. 73, p. 032003, Feb 2006.

[83] R. Field and R. Group, "Pythia tune A, Herwig, and Jimmy in Run 2 at CDF," 2005.

[84] D. Wicke and P. Z. Skands, "Non-perturbative QCD Effects and the Top Mass at the Tevatron," Nuovo Cim., vol. B123, p. S1, 2008.

[85] H. Lai et al., "Global QCD analysis of parton structure of the nucleon: CTEQ5 parton distributions," Eur.Phys.J., vol. C12, pp. 375-392, 2000. 
[86] J. Pumplin et al., "New generation of parton distributions with uncertainties from global QCD analysis," JHEP, vol. 0207, p. 012, 2002.

[87] A. D. Martin, R. Roberts, W. Stirling, and R. Thorne, "Parton distributions: A New global analysis," Eur.Phys.J., vol. C4, pp. 463-496, 1998.

[88] The CDF Collaboration, "Measurement of the Top Pair Cross Section in the Lepton plus Jets decay channel with $2.7 \mathrm{fb}^{-1}$." CDF Conference note: CDF/PUB/TOP/PUBLIC/9462.

[89] G. Mahlon and S. J. Parke, "Angular correlations in top quark pair production and decay at hadron colliders," Phys.Rev., vol. D53, pp. 4886-4896, 1996.

[90] F. A. Berends, H. Kuijf, B. Tausk, and W. T. Giele, "On the production of a W and jets at hadron colliders," Nucl. Phys., vol. B357, pp. 32-64, 1991.

[91] N. Kauer, "Narrow-width approximation limitations," Phys.Lett., vol. B649, pp. 413-416, 2007.

[92] G. P. Lepage, "A New Algorithm for Adaptive Multidimensional Integration," Journal of Computational Physics, vol. 27, pp. 192+, May 1978.

[93] J. T. Linacre, "A Top Quark Mass Measurement Using a Matrix Element Method," FERMILAB-THESIS-2010-05.

[94] B. N. Mohr, "A precise measurement of the top quark mass," FERMILABTHESIS-2007-05.

[95] F. James, "MINUIT: Function Minimization and Error Analysis - Reference Manual." http://wwwasdoc.web.cern.ch/wwwasdoc/minuit/minmain.html

[96] J. Adelman and E. Brubaker, "The Bootstrap Technique and its Application to Analyses in the Top Mass Group." CDF Internal note: CDF/ANAL/TOP/GROUP/9081.

[97] J. Adelman and E. Brubaker, "b-JES Systematic Uncertainties for Top Quark Mass Measurements." CDF Internal Note: CDF/PHYS/TOP/CDFR/9353. 
[98] M. Althoff et al., "Production and muonic decay of heavy quarks in $e^{+} e^{-}$annihilation at $34.5 \mathrm{GeV}$," Zeitschrift für Physik C Particles and Fields, vol. 22, pp. 219-229, 1984. 10.1007/BF01575783.

[99] Y. Peters, K. Hamacher, and D. Wicke, "Precise Tuning of the $b$ Fragmentation for the DØ Monte Carlo," 2009. Fermilab Publication FERMILAB-TM-2425-E.

[100] A. Anastassov et al., "Search for Neutral MSSM Higgs Boson(s) in the tau tau Decay Channel." CDF Internal note: CDF/ANAL/EXOTIC/CDFR/10386.

[101] W. Eadie, Statistical Methods in Experimental Physics. North-Holland, 1988.

[102] R. Field, B. Webber, and M. Seymour, "Comparison of jets in PYTHIA and HERWIG." private communication, 2011.

[103] M. R. Chernick, Bootstrap Methods: a Practitioner's Guide. Wiley series in probability and statistics, Wiley, 1999. 